\title{
Synthesis and Hydrosilylation of Vinylsubstituted Open-Cage Silsesquioxanes with Phenylsilanes: Regioselective Synthesis of Trifunctional Silsesquioxanes
}

\author{
Magdalena Grzelak, ${ }^{1,2 *}$ Rafał Januszewski, ${ }^{1,2}$ Bogdan Marciniec ${ }^{1,2}$ \\ ${ }^{1}$ Faculty of Chemistry, Adam Mickiewicz University in Poznań, \\ Uniwersytetu Poznańskiego 8, 61-614, Poznań, Poland \\ ${ }^{2}$ Center for Advanced Technology, Adam Mickiewicz University in Poznań, \\ Uniwersytetu Poznańskiego 10, 61-614, Poznań, Poland
}

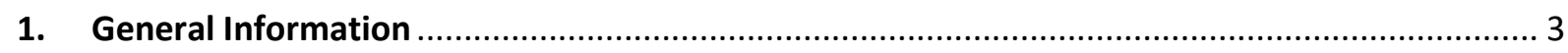

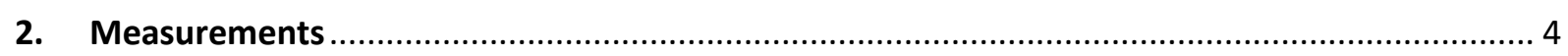

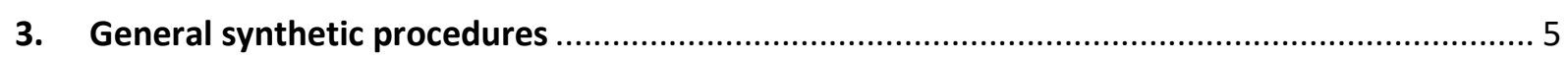

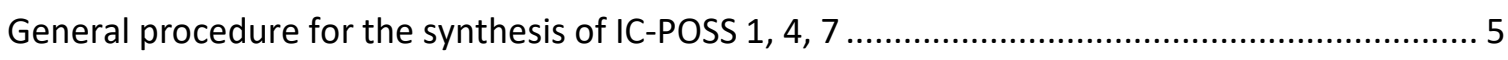

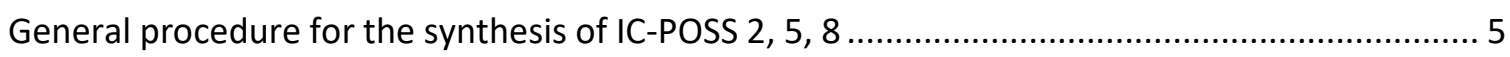

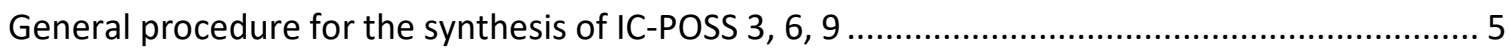

General procedure for hydrosilylation of IC-POSS with phenylsilanes ...................................... 6

4. The ${ }^{1} \mathrm{H} N M R,{ }^{13} \mathrm{C}$ NMR, ${ }^{29}$ Si NMR, and MALDI TOF of IC-POSS 1-9 .................................... 7

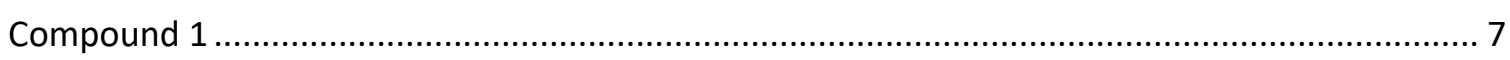

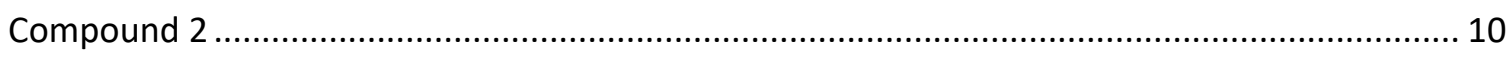

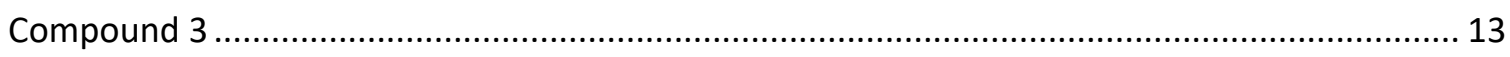

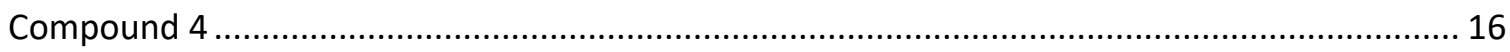

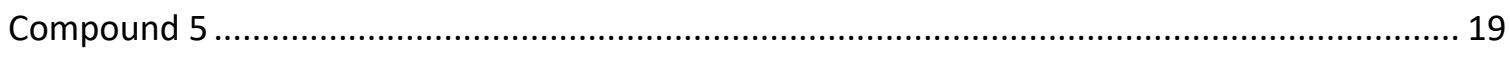

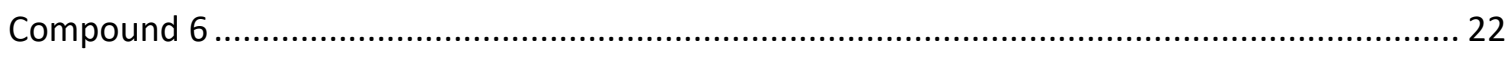

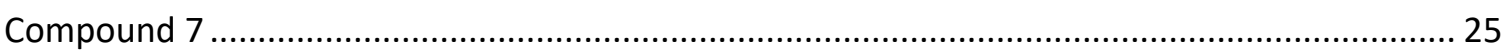

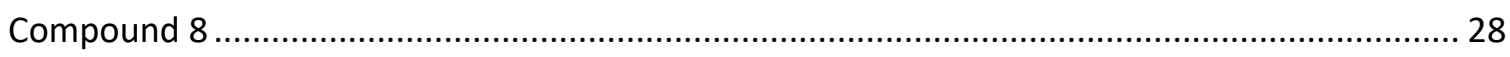

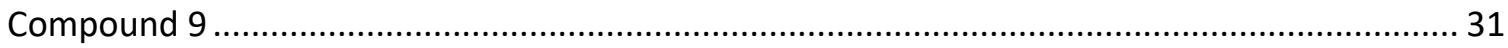


5. The ${ }^{1} \mathrm{H}$ NMR, ${ }^{13} \mathrm{C}$ NMR, ${ }^{29} \mathrm{Si}$ NMR, and MALDI TOF of functionalized IC-POSS .......................... 34

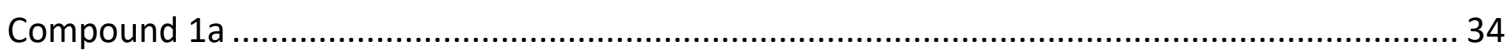

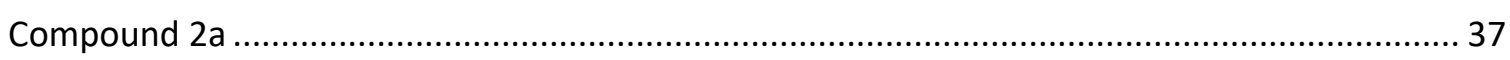

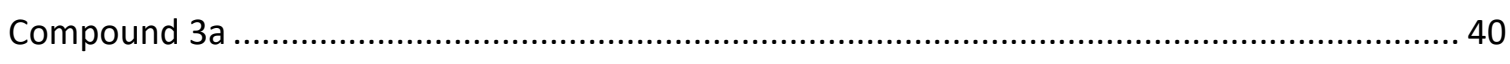

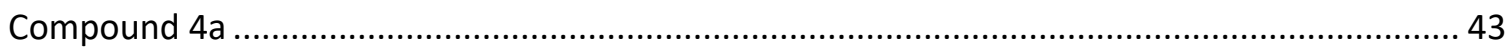

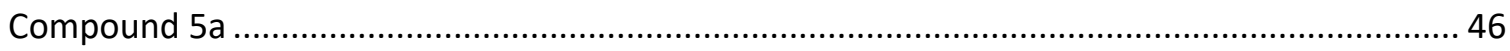

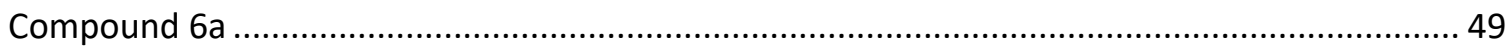

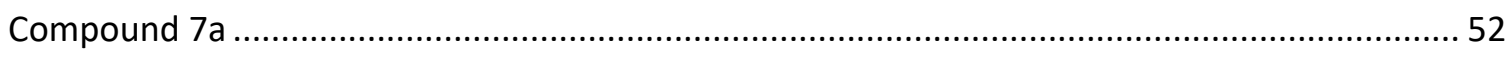

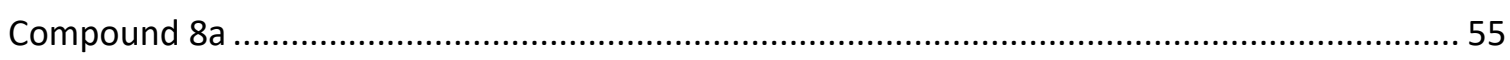

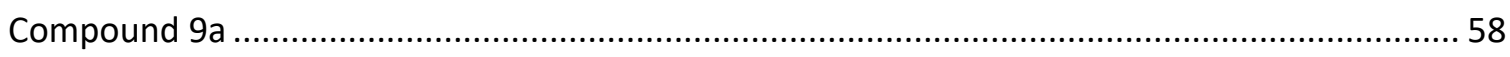

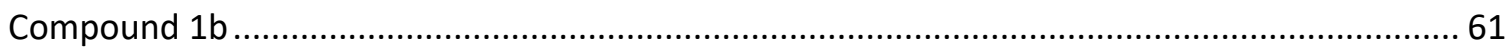

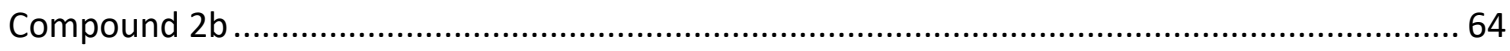

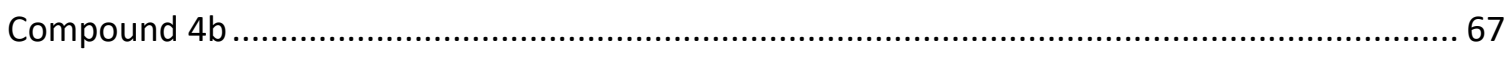

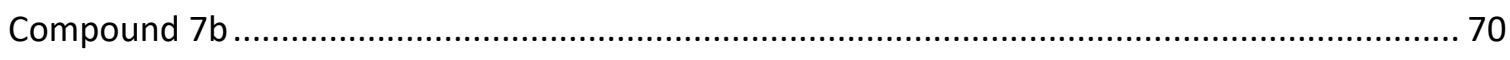

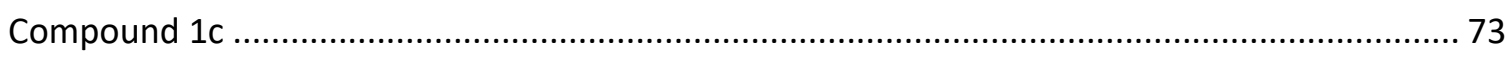

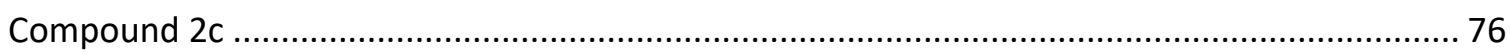

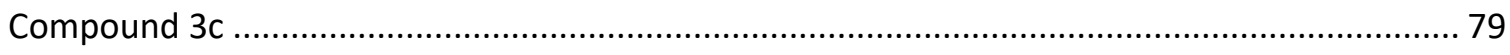

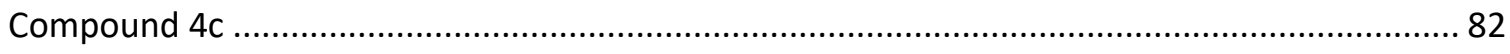

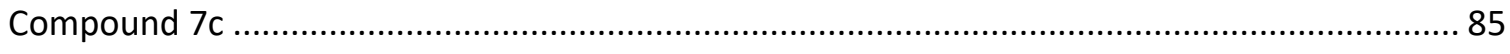

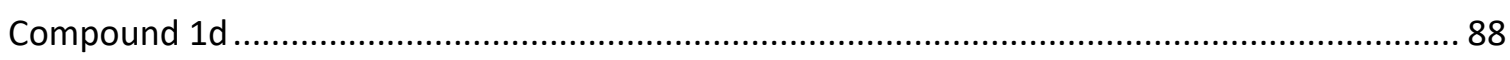

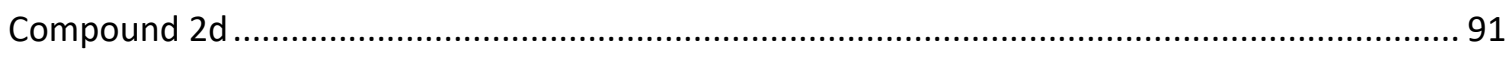

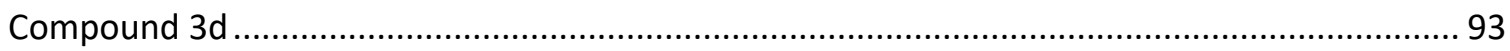

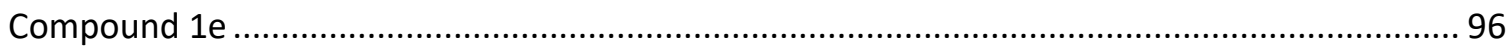

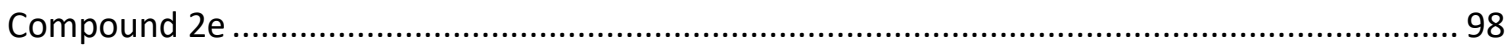

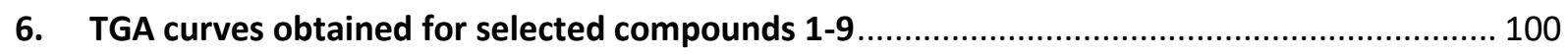




\section{General Information}

The chemicals were purchased from the following sources: chlorodimethylvinylsilane (97\%), chloromethylphenylvinylsilane (95\%), chlorodiphenylvinylsilane (90\%), chlorodimethylsilane (98\%), 4bromobiphenyl (98\%), 1,4-dibromobenzene (98\%), n-butyllithium solution $2.5 \mathrm{M}$ in hexanes, dimethylphenylsilane (98\%), triphenylsilane (97\%), 1,4-bis(dimethylsilyl)benzene (97\%), triethylamine, Karstedt's catalyst $\left[\mathrm{Pt}_{2}(\mathrm{dvs})_{3}\right], \mathrm{H}_{2} \mathrm{PtCl}_{6},\left[\mathrm{Rh}(\mathrm{COD}) \mathrm{Cl}_{2}, \mathrm{PtO}_{2}\right.$, dichloromethane (DCM), ethyl acetate, tetrahydrofuran (THF), toluene, magnesium sulphate, silica gel $60 \AA$, Celite ${ }^{\circledR}$ from Aldrich; Trisilanollsobutyl POSS, TrisilanolPhenyl POSS, Trisilanollsooctyl POSS from Hybrid Plastics, $\mathrm{CDCl}_{3}$ from Deutero.

Condensation reactions and synthesis of silanes $\mathbf{c}$ and $\mathbf{d}$ were carried out under argon atmosphere using standard Schlenk-line and vacuum techniques. THF was dried with sodium benzophenone ketyl and freshly distilled before use. Chlorodimethylvinylsilane, chloromethylphenylvinylsilane, chlorodiphenylvinylsilane, chlorodimethylsilane and triethylamine were purified by trap-to-trap distillation. THF, triethylamine and silanes were stored under argon.

Silanes $\mathbf{c}\left(\left(1,1^{\prime} \text {-biphenyl-4-yl)dimethylsilane }\right)^{1} \text { and } \mathbf{d} \text { ((4-bromophenyl)dimethylsilane }\right)^{2}$ were prepared according to literature procedures.

(1) Ohshita, J.; Sugimoto, K.; Watanabe, T.; Kunai, A.; Ishikawa, M.; Aoyama, S. Polymeric Organosilicon Systems. XXIX. Thermal Properties of Poly[(Disilanylene)Oligophenylenes]. J. Organomet. Chem. 1998, 564 (1-2), 47-56.

(2) Rayment, E. J.; Summerhill, N.; Anderson, E. A. Synthesis of Phenols via Fluoride-Free Oxidation of Arylsilanes and Arylmethoxysilanes. J. Org. Chem. 2012, 77 (16), 7052-7060. 


\section{Measurements}

\section{Nuclear magnetic resonance spectroscopy (NMR)}

The ${ }^{1} \mathrm{H}(300 \mathrm{MHz}),{ }^{13} \mathrm{C}(101 \mathrm{MHz})$, and ${ }^{29} \mathrm{Si} \mathrm{NMR}(79 \mathrm{MHz})$ spectra were recorded with a Varian XL 300 $\mathrm{MHz}$ spectrometer and Varian VNMR-S $400 \mathrm{MHz}$ spectrometer with samples in $\mathrm{CDCl}_{3}$ solution. The chemical shifts are reported in ppm and were referenced to the residual solvent signals $(\delta \mathrm{H}=7.26$ $\mathrm{ppm}, \delta \mathrm{C}=77.36 \mathrm{ppm}$ for $\mathrm{CDCl}_{3}$ ).

\section{In situ FT-IR spectroscopy}

Real-time FT-IR measurements were performed on a Mettler Toledo ReactIR 15 equipped with a DS $6.3 \mathrm{~mm}$ AgXDiComp Fiber Probe with a diamond sensor, and a Mercury Cadmium Telluride detector. For all the spectra 256 scans were recorded with the resolution of $1 \mathrm{~cm}^{-1}$ in 1,5 and 10 min intervals.

\section{FT-IR spectroscopy}

FT-IR spectra were performed on a Nicolet iS50 FT-IR Spectrometer equipped with a SPECAC Golden Gate. For all spectra, 16 scans at a resolution of $2 \mathrm{~cm}^{-1}$ were collected. Samples were measured as obtained, without any preparatory steps.

\section{Matrix-assisted laser desorption/ionization time-of-flight mass spectroscopy (MALDI-TOF MS)} MALDI-TOF mass spectra were recorded on a UltrafleXtreme mass spectrometer (Bruker Daltonics), equipped with a SmartBeam II laser $(355 \mathrm{~nm}$ ) in 500-4000 m/z range. 2,5-Dihydroxybenzoic acid (DHB) served as matrix.

\section{Thermogravimetric Analyses (TGA)}

TGA were performed using a TGA/DSC 1 Mettler-Toledo thermal gravimetric analyzer. The measurements were conducted in a air atmosphere (flow of $60 \mathrm{~mL} / \mathrm{min}$ ), from ambient temperature to $1000^{\circ} \mathrm{C}$ at the heating rate of $10^{\circ} \mathrm{C} / \mathrm{min}$. The temperature of initial degradation (T5\%) was taken as the onset temperature at which $5 \mathrm{wt} \%$ of mass loss occurs. 


\section{General synthetic procedures}

General procedure for the synthesis of IC-POSS 1, 4, 7

A two-necked, $500 \mathrm{~mL}$ flask equipped with a magnetic stirring bar, reflux condenser and connected to gas and vacuum line was charged under argon with Trisilanollsobutyl POSS (10 g, $12.63 \mathrm{mmol}$ ), tetrahydrofuran $(200 \mathrm{~mL})$, and triethylamine $(10.57 \mathrm{~mL}, 75.78 \mathrm{mmol})$. The reaction mixture was cooled in an ice bath and then, chlorodimethylvinylsilane for $\mathbf{1}$, chloromethylphenylvinylsilane for $\mathbf{4}$, and chlorodiphenylvinylsilane for $\mathbf{7}(39.16 \mathrm{mmol})$ was added dropwise and the cooling bath left to warm up to room temperature. The molar ratio of the reactants was as follows: [silanol POSS] : [chlorovinylmetalloid] : $\left[\mathrm{Et}_{3} \mathrm{~N}\right]=[1]:[3.1]:[6]$. Progress of the reaction was followed by FT-IR spectroscopy. After reaction, the mixture were filtered off by glass filter type $\mathrm{G} 4$ with silica-gel and celite and then the column was flushed by ethyl acetate to rewash all product. The excess of solvent was evaporated and product was dried under vacuum.

General procedure for the synthesis of IC-POSS 2, 5, 8

A two-necked, $500 \mathrm{~mL}$ flask equipped with a magnetic stirring bar, reflux condenser and connected to gas and vacuum line was charged under argon with TrisilanolPhenyl POSS (10 g, $10.74 \mathrm{mmol}$ ), tetrahydrofuran $(250 \mathrm{~mL})$, and triethylamine $(8.98 \mathrm{~mL}, 64.44 \mathrm{mmol})$. The reaction mixture was cooled in an ice bath and then, chlorodimethylvinylsilane for $\mathbf{2}$, chloromethylphenylvinylsilane for $\mathbf{5}$, and chlorodiphenylvinylsilane for $\mathbf{8}(33.29 \mathrm{mmol})$ was added dropwise and the cooling bath left to warm up to room temperature. The molar ratio of the reactants was as follows: [silanol POSS] : [chlorovinylmetalloid] : $\left[\mathrm{Et}_{3} \mathrm{~N}\right]=[1]:[3.1]:[6]$. Progress of the reaction was followed by FT-IR spectroscopy. After reaction, the mixture were filtered off by glass filter type G4 with silica-gel and celite and then the column was flushed by ethyl acetate to rewash all product. The excess of solvent was evaporated and product was dried under vacuum.

\section{General procedure for the synthesis of IC-POSS 3, 6, 9}

A two-necked, $500 \mathrm{~mL}$ flask equipped with a magnetic stirring bar, reflux condenser and connected to gas and vacuum line was charged under argon with Trisilanollsooctyl POSS (10 g, 8.45 mmol), tetrahydrofuran $(200 \mathrm{~mL})$, and triethylamine $(7.06 \mathrm{~mL}, 50.7 \mathrm{mmol})$. The reaction mixture was cooled in an ice bath and then, chlorodimethylvinylsilane for $\mathbf{3}$, chloromethylphenylvinylsilane for $\mathbf{6}$, and chlorodiphenylvinylsilane for $\mathbf{9}(26.2 \mathrm{mmol})$ was added dropwise and the cooling bath left to warm up to room temperature. The molar ratio of the reactants was as follows: [silanol POSS] : [chlorovinylmetalloid] : $\left[\mathrm{Et}_{3} \mathrm{~N}\right]=[1]:[3.1]:[6]$. Progress of the reaction was followed by FT-IR spectroscopy. After reaction, the mixture were filtered off by glass filter type G4 with silica-gel and 
celite and then the column was flushed by ethyl acetate to rewash all product. The solution after filtration was evaporated and product was extracted with $\mathrm{DCM} / \mathrm{H}_{2} \mathrm{O}$ and then the organic phase was dried over anhydrous magnesium sulfate for $24 \mathrm{~h}$, filtered and the solvent was evaporated and product was dried under vacuum.

General procedure for hydrosilylation of IC-POSS with phenylsilanes

To a $5 \mathrm{~mL}$ glass reactor IC-POSS (200 mg, 1.0 eq.) of 1-9, silane (3.0 eq.of silane $\mathbf{a}, \mathbf{b}, \mathbf{c}, \mathbf{d}$ and 3.1 eq of silane e) and toluene $(2 \mathrm{~mL})$ were added. The reaction mixture was stirred at $95^{\circ} \mathrm{C}$ for few minutes. After this time, the Karstedt's catalyst $3 \times 10^{-4}$ mol of Pt per 1 mole of POSS was added. After reaction, the mixture was filtered off by glass filter type G4 with silica-gel and Celite, the solvent was evaporated and excess was removed under reduced pressure to give products. 


\section{The ${ }^{1} \mathrm{H}$ NMR, ${ }^{13} \mathrm{C}$ NMR, ${ }^{29} \mathrm{Si}$ NMR, and MALDI TOF of IC-POSS 1-9}

Compound 1

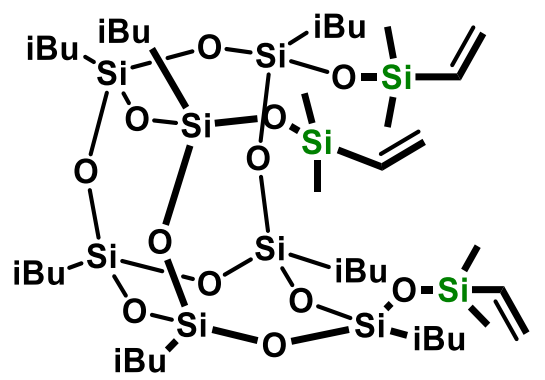

Chemical Formula: $\mathrm{C}_{40} \mathrm{H}_{90} \mathrm{O}_{12} \mathrm{Si}_{10}$

${ }^{1} \mathrm{H}$ NMR (300 MHz, Chloroform-d) $\delta 6.15$ (dd, $J=20.2,14.8 \mathrm{~Hz}, 3 \mathrm{H}$ ), 5.92 (dd, $J=14.8,4.1 \mathrm{~Hz}, 3 \mathrm{H}$ ), 5.74 (dd, $J=20.2,4.1 \mathrm{~Hz}, 3 \mathrm{H}), 1.91-1.74(\mathrm{~m}, 7 \mathrm{H}), 0.95(\mathrm{dd}, J=6.6,3.4 \mathrm{~Hz}, 42 \mathrm{H}), 0.56$ (dd, $J=7.1,1.3 \mathrm{~Hz}$, $14 \mathrm{H}), 0.20(\mathrm{~s}, 18 \mathrm{H})$.

${ }^{13} \mathrm{CNMR}\left(75 \mathrm{MHz}, \mathrm{CDCl}_{3}\right) \delta$ 139.31, 131.91, 26.18, 26.02, 25.78, 25.12, 24.21, 24.10, 23.88, 22.61, 0.53.

${ }^{29} \mathrm{Si} \mathrm{NMR}\left(79 \mathrm{MHz}, \mathrm{CDCl}_{3}\right) \delta-2.68,-67.29,-67.81,-68.04$.

MALDI-TOF MS (m/z): calcd. for $\mathrm{C}_{40} \mathrm{H}_{90} \mathrm{O}_{12} \mathrm{Si}_{10} \mathrm{Na}$ 1065,40; found 1065,40.

\section{${ }^{1} \mathrm{H}$ NMR $\left(300 \mathrm{MHz}, \mathrm{CDCl}_{3}\right)$}

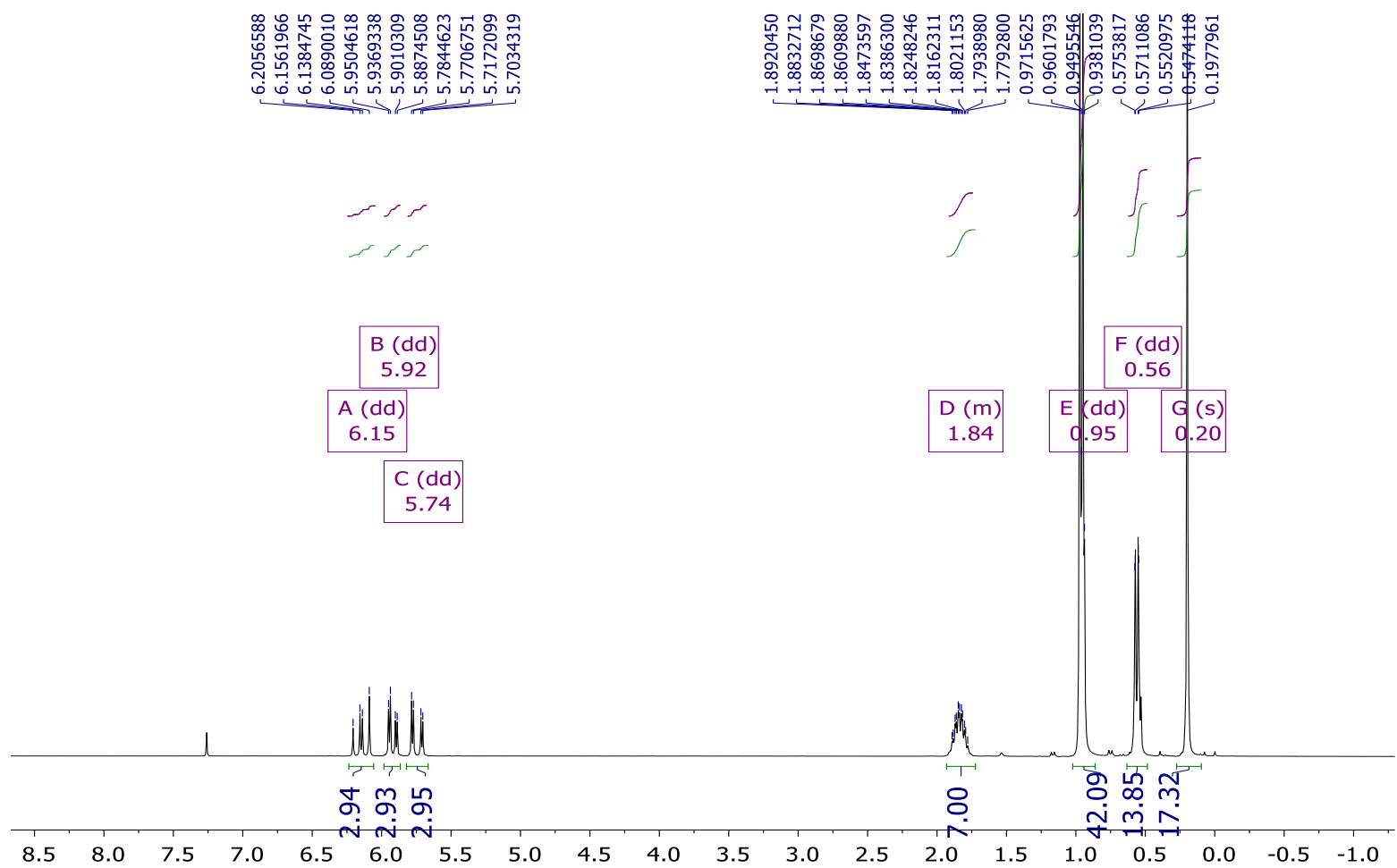


${ }^{13} \mathrm{C}$ NMR (101 MHz, $\mathrm{CDCl}_{3}$ )

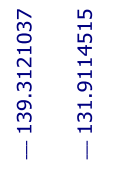

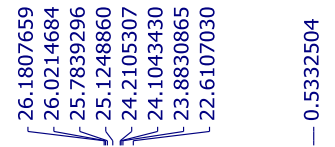

$\mid$

$\begin{array}{lllllllllllllllllll}160 & 150 & 140 & 130 & 120 & 110 & 100 & 90 & 80 & 70 & 60 & 50 & 40 & 30 & 20 & 10 & 0 & -10 & -20\end{array}$

${ }^{29}$ Si NMR (79 MHz, $\mathrm{CDCl}_{3}$ )

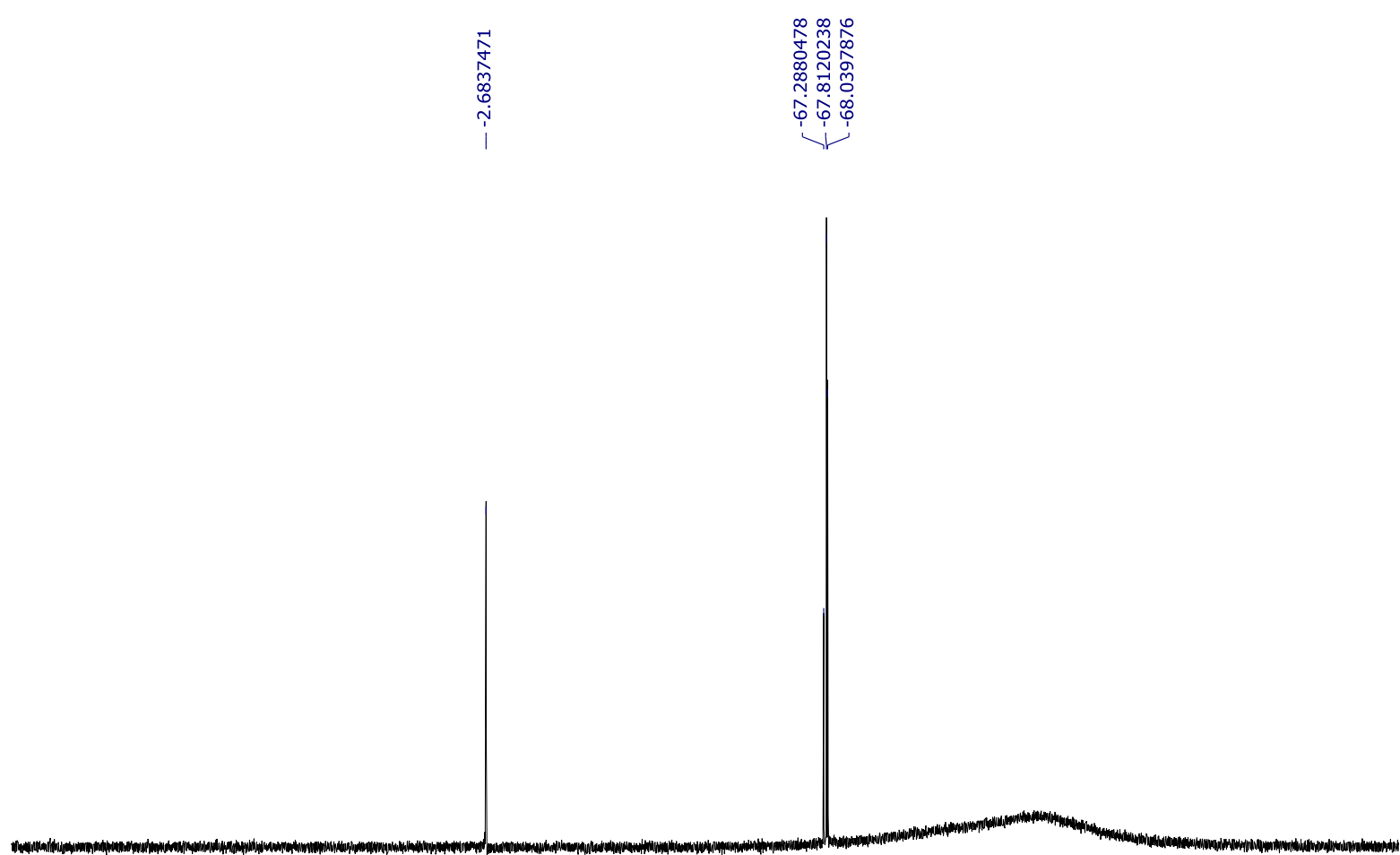


MALDI-TOF MS

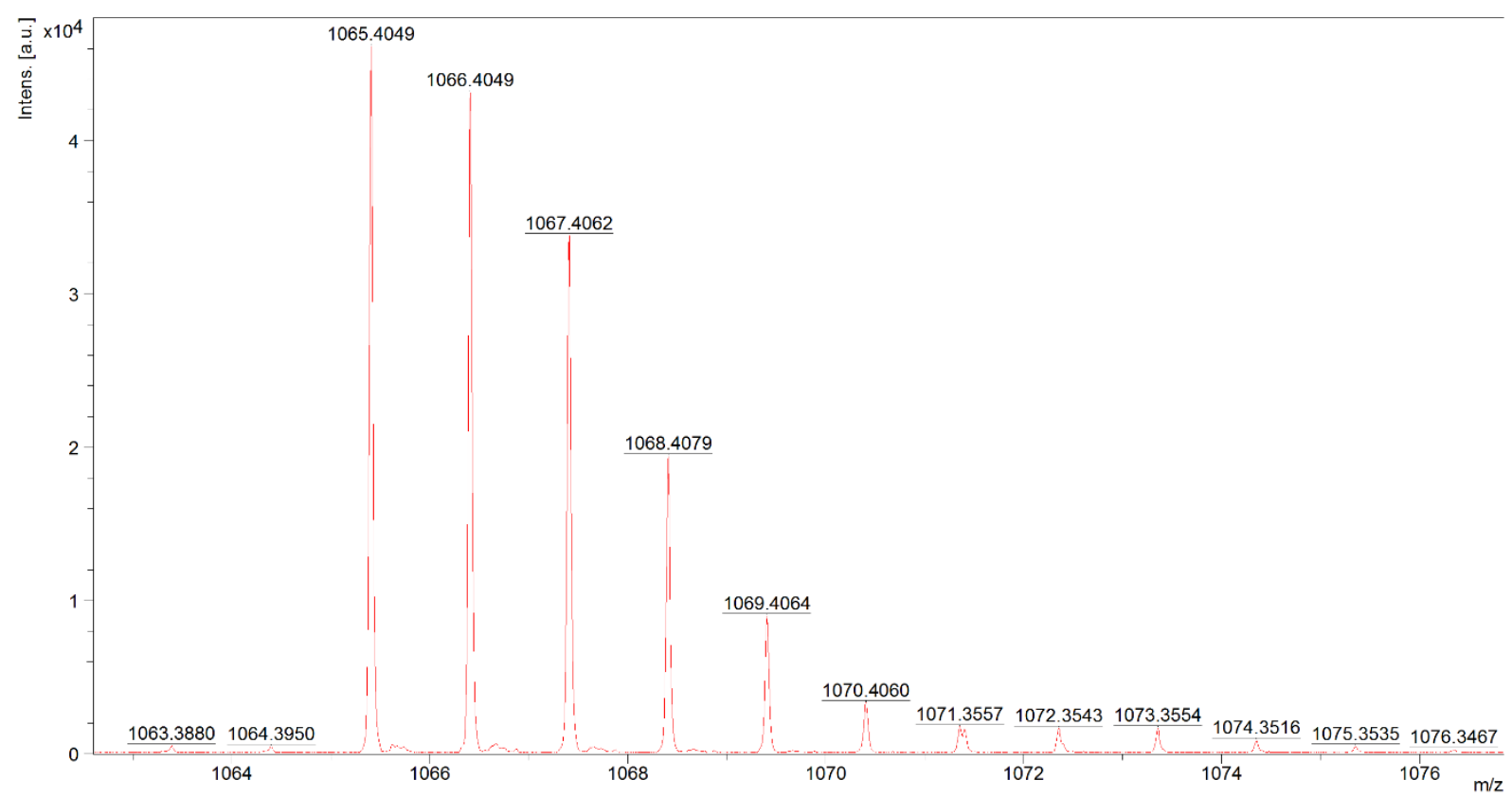


Compound 2

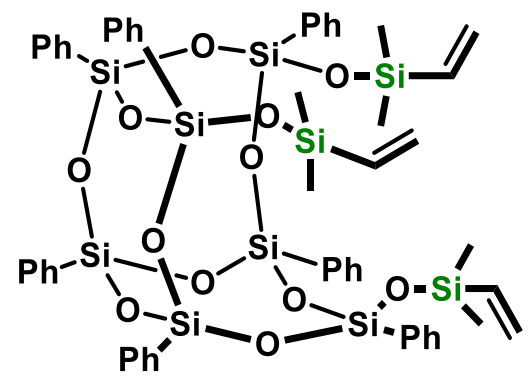

Chemical Formula: $\mathrm{C}_{54} \mathrm{H}_{62} \mathrm{O}_{12} \mathrm{Si}_{10}$

${ }^{1} \mathrm{H}$ NMR (300 MHz, Chloroform-d) $\delta 7.46-7.37(\mathrm{~m}, 7 \mathrm{H}), 7.37-7.28(\mathrm{~m}, 15 \mathrm{H}), 7.19-7.07(\mathrm{~m}, 13 \mathrm{H})$, 6.22 (ddt, $J=20.1,14.9,2.0 \mathrm{~Hz}, 3 \mathrm{H}$ ), 5.98 (ddt, $J=14.9,3.9,1.9 \mathrm{~Hz}, 3 \mathrm{H}$ ), 5.84 (ddt, $J=20.2,4.0,2.0 \mathrm{~Hz}$, $3 \mathrm{H}), 0.32(\mathrm{~s}, 18 \mathrm{H})$.

${ }^{13} \mathrm{C} \mathrm{NMR}\left(75 \mathrm{MHz}, \mathrm{CDCl}_{3}\right) \delta 138.75,134.17,134.13,132.89,132.55,131.27,130.24,130.08,128.02$, $127.68,127.61,0.53$.

${ }^{29} \mathrm{Si}$ NMR $\left(79 \mathrm{MHz}, \mathrm{CDCl}_{3}\right) \delta-0.16,-77.40,-77.79,-78.15$.

MALDI-TOF MS (m/z): calcd. for $\mathrm{C}_{54} \mathrm{H}_{62} \mathrm{O}_{12} \mathrm{Si}_{10} \mathrm{Na} 1205,18$; found 1205,18.

\section{${ }^{1} \mathrm{H}$ NMR $\left(300 \mathrm{MHz}, \mathrm{CDCl}_{3}\right)$}
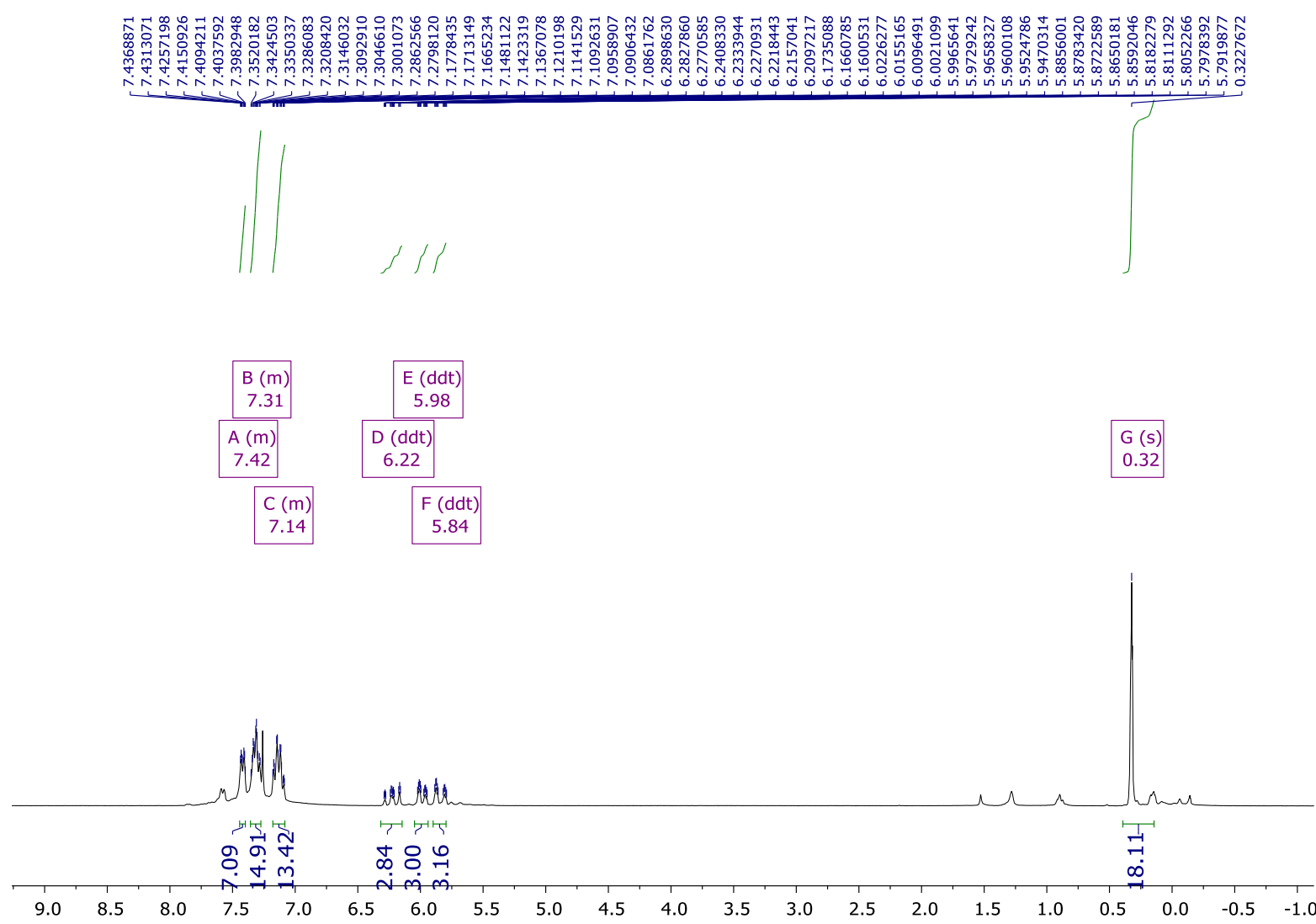
${ }^{13} \mathrm{C}$ NMR (101 MHz, $\mathrm{CDCl}_{3}$ )

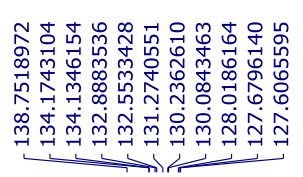

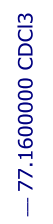

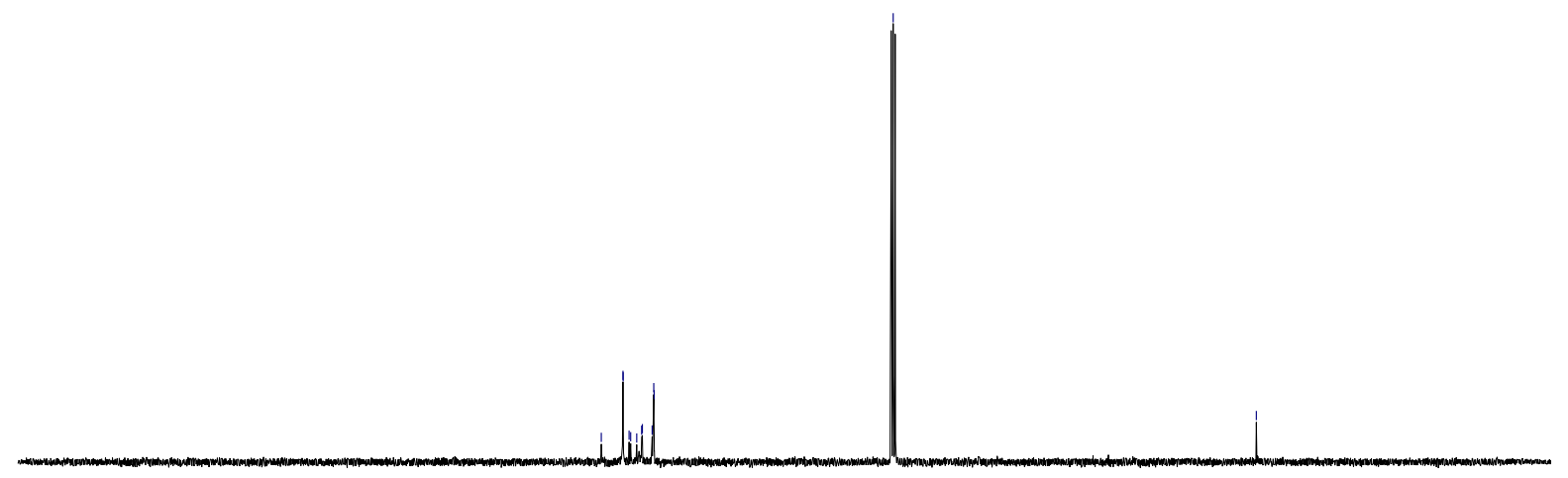

${ }^{29} \mathrm{Si} \mathrm{NMR}\left(79 \mathrm{MHz}, \mathrm{CDCl}_{3}\right)$
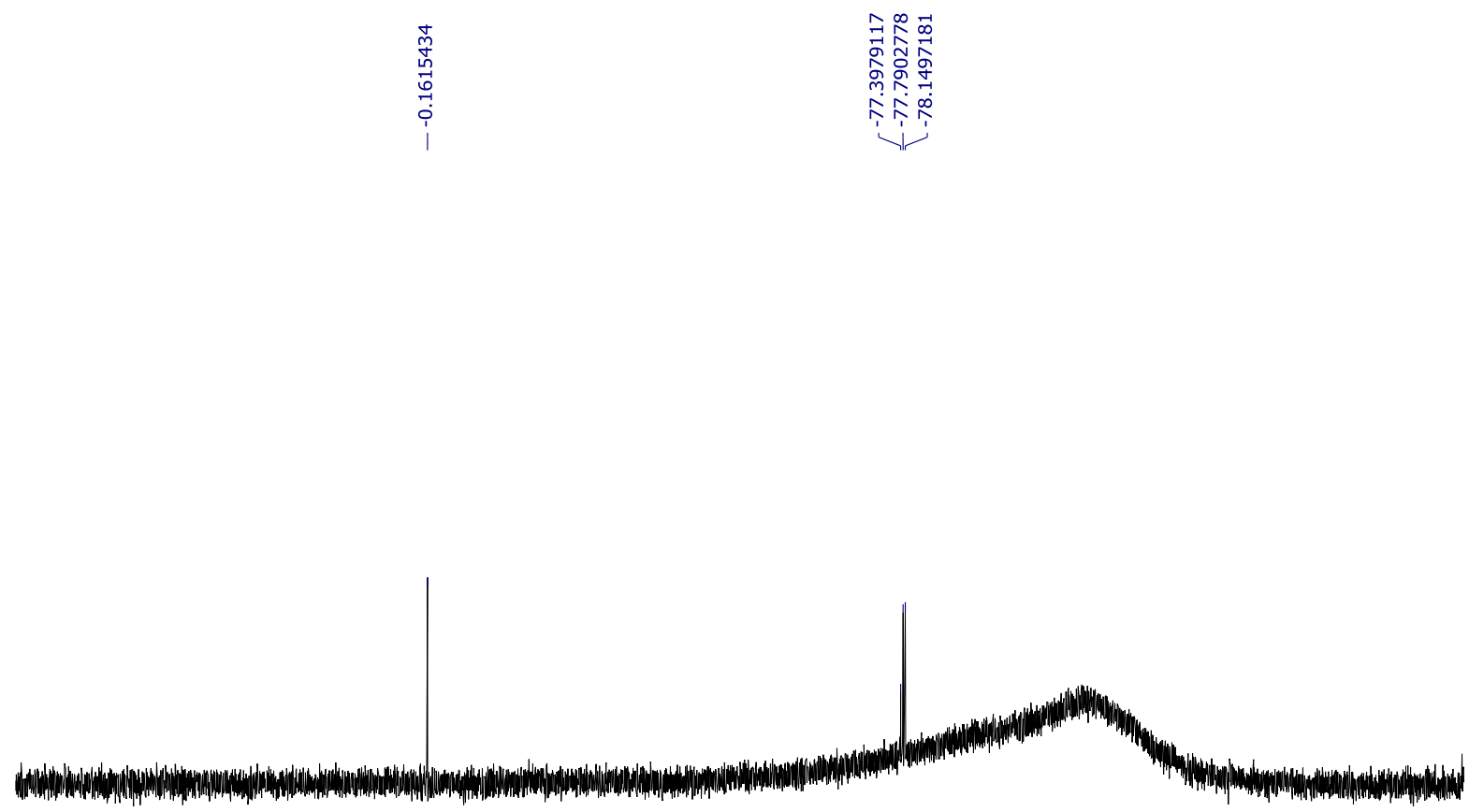
MALDI-TOF MS

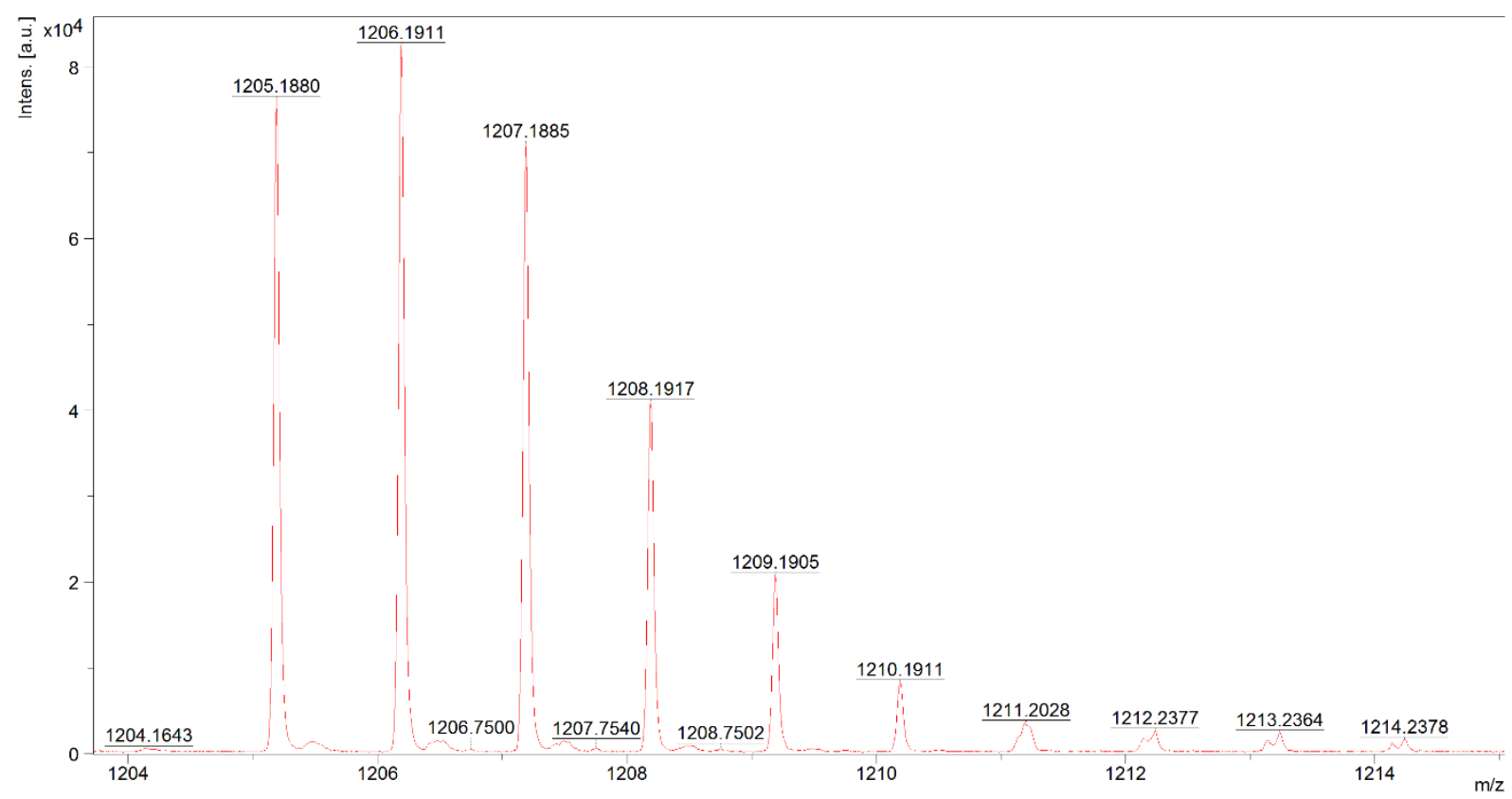


Compound 3

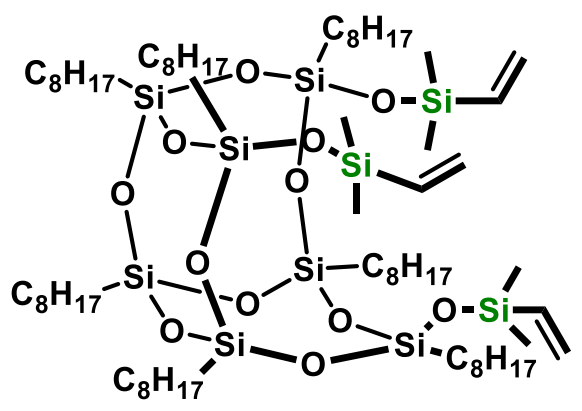

Chemical Formula: $\mathrm{C}_{68} \mathrm{H}_{146} \mathrm{O}_{12} \mathrm{Si}_{10}$

${ }^{1} \mathrm{H}$ NMR $(300 \mathrm{MHz}$, Chloroform- $d) \delta 6.15$ (ddt, $\left.J=20.5,14.9,3.0 \mathrm{~Hz}, 3 \mathrm{H}\right), 5.97-5.87(\mathrm{~m}, 3 \mathrm{H}), 5.74$ (ddt, $J=20.2,4.3,2.3 \mathrm{~Hz}, 3 \mathrm{H}), 1.90-1.72(\mathrm{~m}, 7 \mathrm{H}), 1.37-1.08(\mathrm{~m}, 15 \mathrm{H}), 1.05-0.95(\mathrm{~m}, 20 \mathrm{H}), 0.90(\mathrm{~d}, J=2.6$ $\mathrm{Hz}, 63 \mathrm{H}), 0.82-0.64(\mathrm{~m}, 7 \mathrm{H}), 0.54(\mathrm{dt}, J=14.5,8.7 \mathrm{~Hz}, 7 \mathrm{H}), 0.20(\mathrm{~s}, 18 \mathrm{H})$.

${ }^{13} \mathrm{C} \mathrm{NMR}\left(75 \mathrm{MHz}, \mathrm{CDCl}_{3}\right) \delta 139.35,131.93,55.19,54.60,31.34,30.43,26.47,25.82,25.47,25.36,0.62$. ${ }^{29} \mathrm{Si} \mathrm{NMR}\left(79 \mathrm{MHz}, \mathrm{CDCl}_{3}\right) \delta-2.75,-2.84,-2.95,-67.51,-67.86,-67.98,-68.02,-68.06,-68.24,-68.32,-$ 68.36, -68.52 .

MALDI-TOF MS (m/z): calcd. for $\mathrm{C}_{68} \mathrm{H}_{14} 6 \mathrm{O}_{12} \mathrm{Si}_{10} \mathrm{Na} 1457,84$; found 1457,84 .

\section{${ }^{1} \mathrm{H}$ NMR $\left(300 \mathrm{MHz}, \mathrm{CDCl}_{3}\right)$}

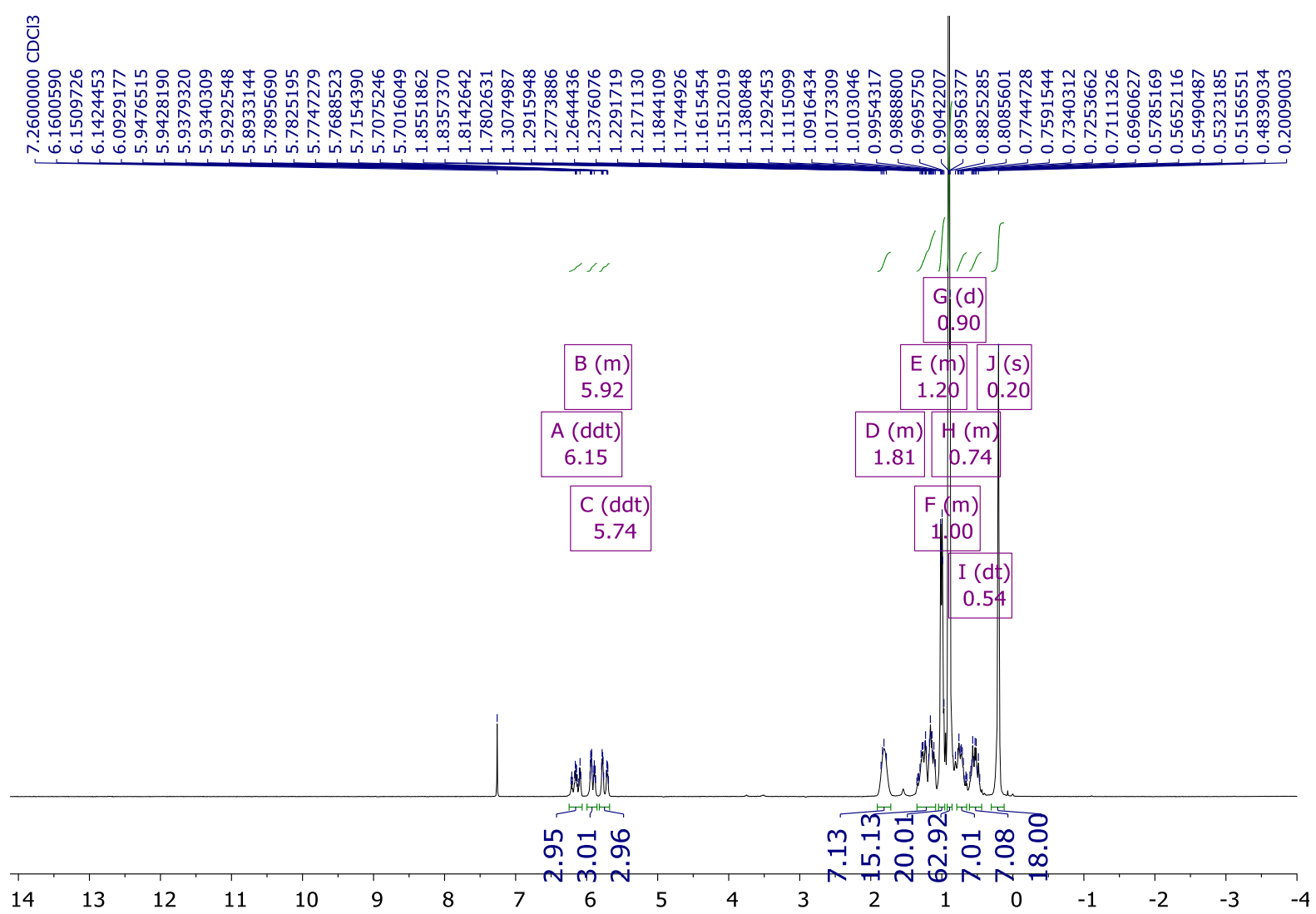


$\left.{ }^{13} \mathrm{C} \mathrm{NMR} \mathrm{(101} \mathrm{MHz,} \mathrm{CDCl}_{3}\right)$

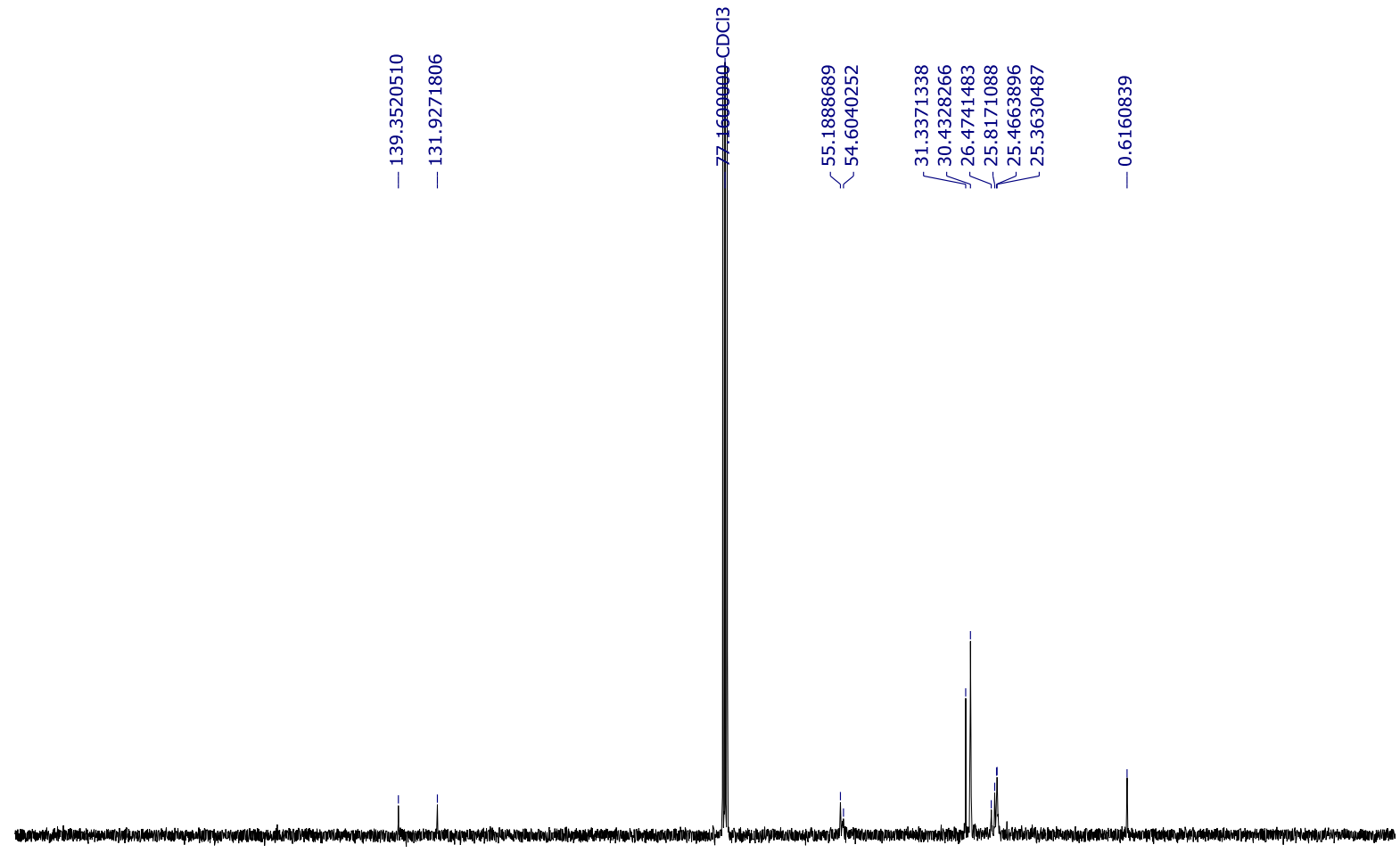

$\begin{array}{lllllllllllllllllllllllllll}210 & 200 & 190 & 180 & 170 & 160 & 150 & 140 & 130 & 120 & 110 & 100 & 90 & 80 & 70 & 60 & 50 & 40 & 30 & 20 & 10 & 0 & -10 & -20 & -30 & -40 & -5(\end{array}$ ${ }^{29} \mathrm{Si} \mathrm{NMR}\left(79 \mathrm{MHz}, \mathrm{CDCl}_{3}\right)$
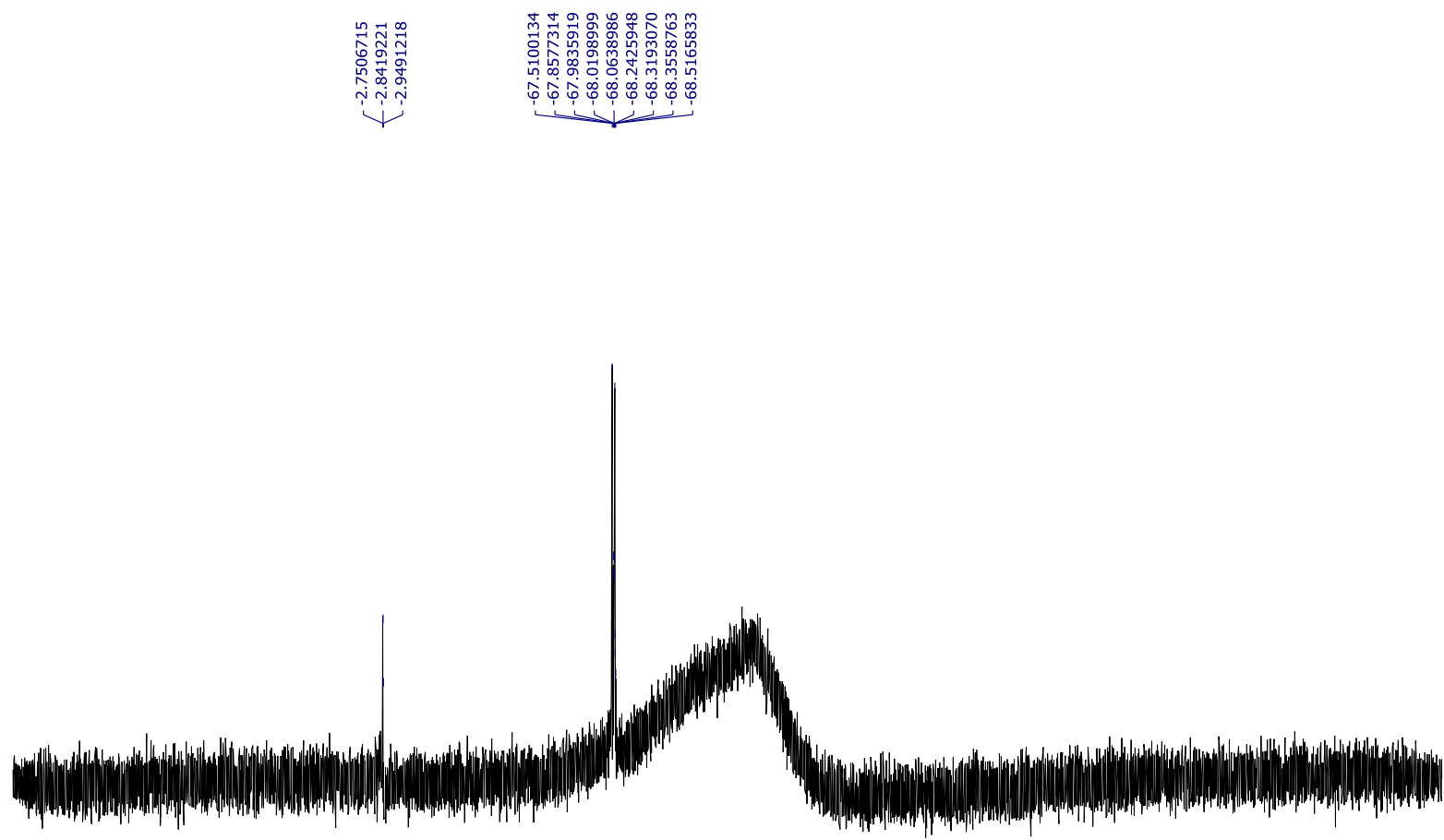

$\begin{array}{llllllllllllllllllllll}100 & 80 & 60 & 40 & 20 & 0 & -20 & -40 & -60 & -80 & -100 & -120 & -140 & -160 & -180 & -200 & -220 & -240 & -260 & -280 & -301\end{array}$ 
MALDI-TOF MS

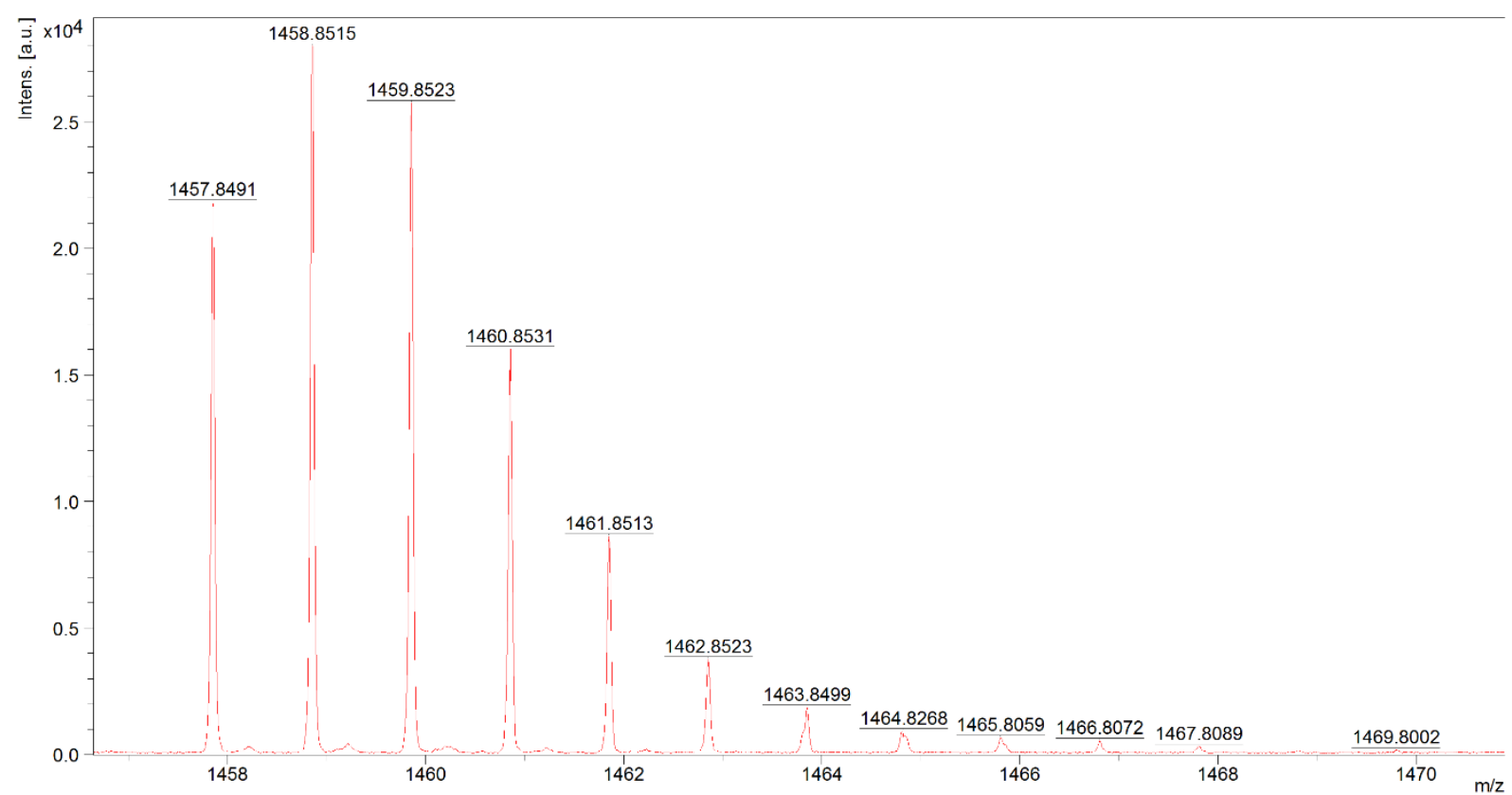


Compound 4

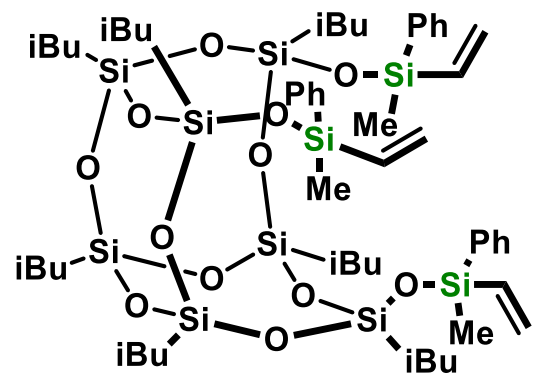

Chemical Formula: $\mathrm{C}_{55} \mathrm{H}_{96} \mathrm{O}_{12} \mathrm{Si}_{10}$

${ }^{1} \mathrm{H}$ NMR $(300 \mathrm{MHz}$, Chloroform-d) $\delta 7.68-7.48(\mathrm{~m}, 6 \mathrm{H}), 7.39-7.27(\mathrm{~m}, 9 \mathrm{H}), 6.20$ (ddt, $J=20.6,14.9$, $6.2 \mathrm{~Hz}, 3 \mathrm{H}$ ), 5.97 (dddd, $J=14.9,9.0,7.1,4.0 \mathrm{~Hz}, 3 \mathrm{H}$ ), 5.78 (dddd, $J=20.2,6.8,5.5,4.0 \mathrm{~Hz}, 3 \mathrm{H}$ ), $1.98-$ $1.72(\mathrm{~m}, 7 \mathrm{H}), 1.07-0.86(\mathrm{~m}, 42 \mathrm{H}), 0.65-0.52(\mathrm{~m}, 14 \mathrm{H}), 0.39(\mathrm{t}, J=7.4 \mathrm{~Hz}, 9 \mathrm{H})$.

${ }^{13} \mathrm{C} \mathrm{NMR}\left(75 \mathrm{MHz}, \mathrm{CDCl}_{3}\right) \delta 137.85,137.06,134.06,133.88,129.40,127.69,26.17,26.05,25.78,25.11$, $24.11,23.87,22.61,-0.77$.

${ }^{29} \mathrm{Si}$ NMR $\left(79 \mathrm{MHz}, \mathrm{CDCl}_{3}\right) \delta-12.59,-67.23,-67.40,-67.51$.

MALDI-TOF MS (m/z): calcd. for $\mathrm{C}_{55} \mathrm{H}_{96} \mathrm{O}_{12} \mathrm{Si}_{10} \mathrm{Na}$ 1251,45; found 1251,45.

\section{${ }^{1} \mathrm{H}$ NMR $\left(300 \mathrm{MHz}, \mathrm{CDCl}_{3}\right)$}

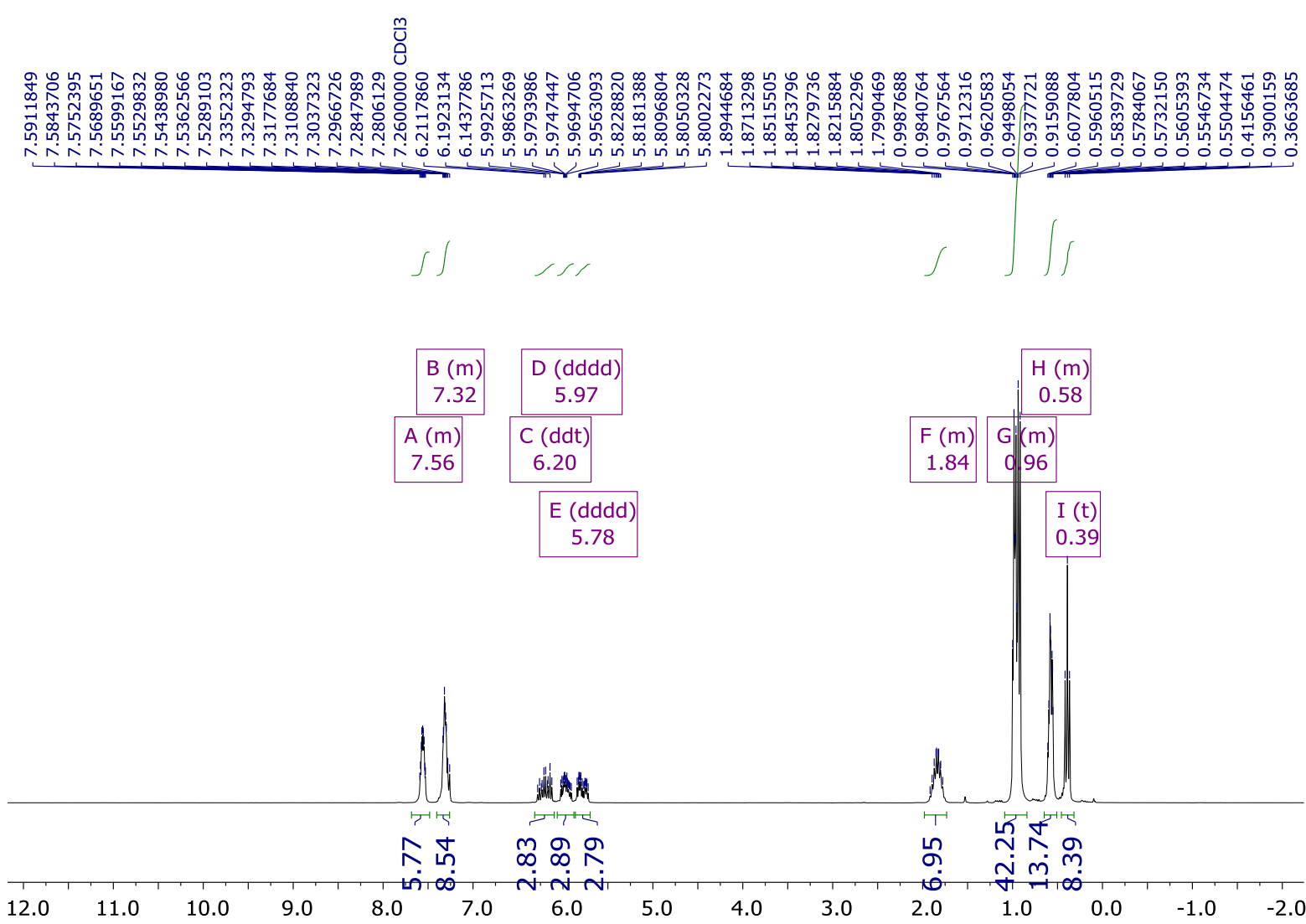


${ }^{13} \mathrm{C}$ NMR (101 MHz, $\mathrm{CDCl}_{3}$ )

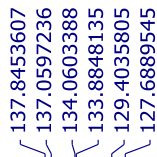

$$
\begin{aligned}
& m \\
& 0 \\
& 0 \\
& 0 \\
& o \\
& 0 \\
& 0 \\
& 0 \\
& i \\
& i
\end{aligned}
$$
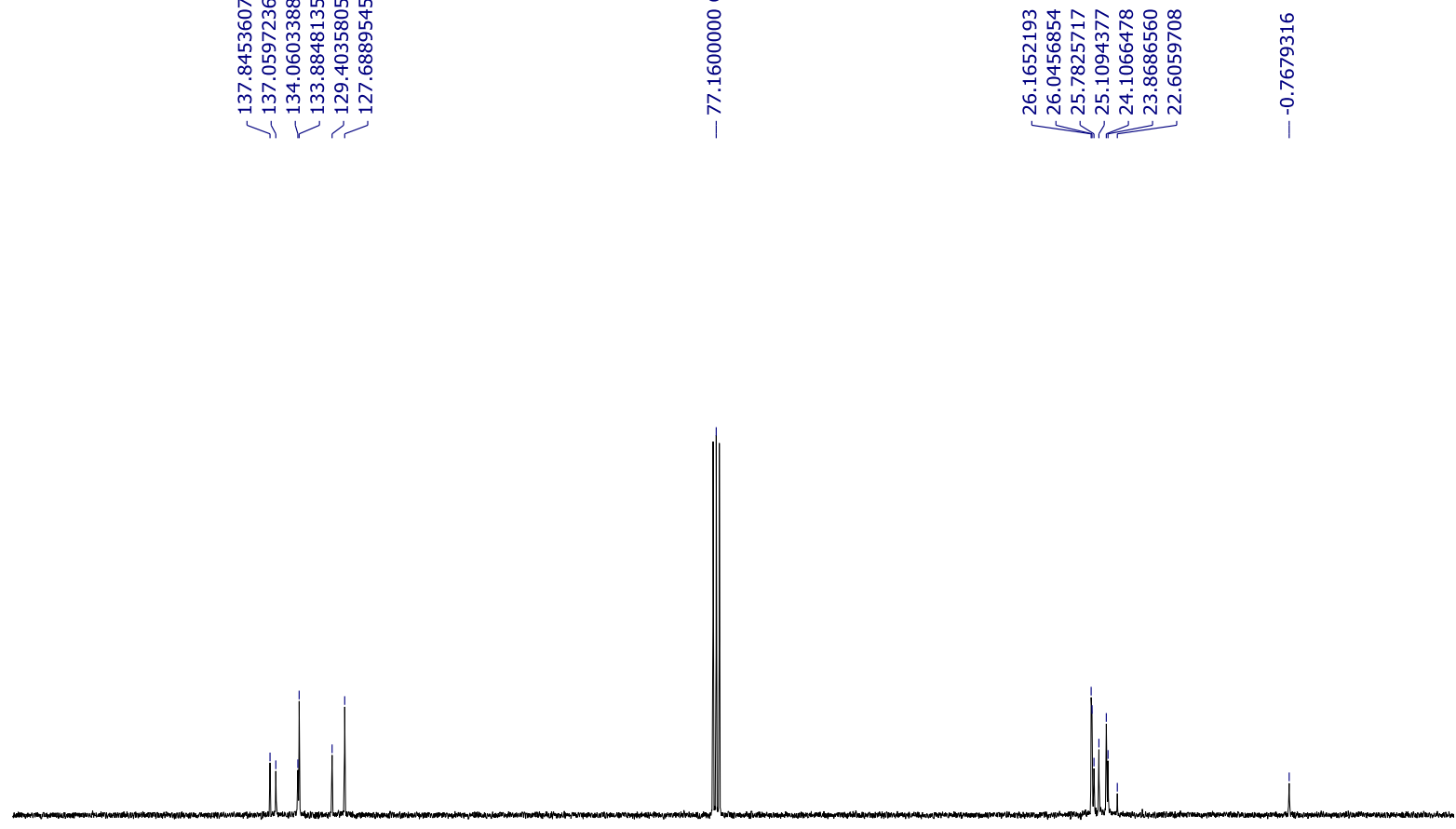

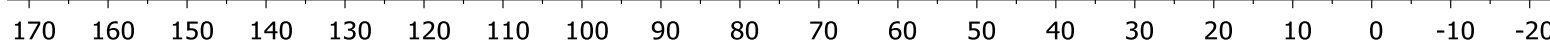

${ }^{29}$ Si NMR (79 MHz, $\mathrm{CDCl}_{3}$ )
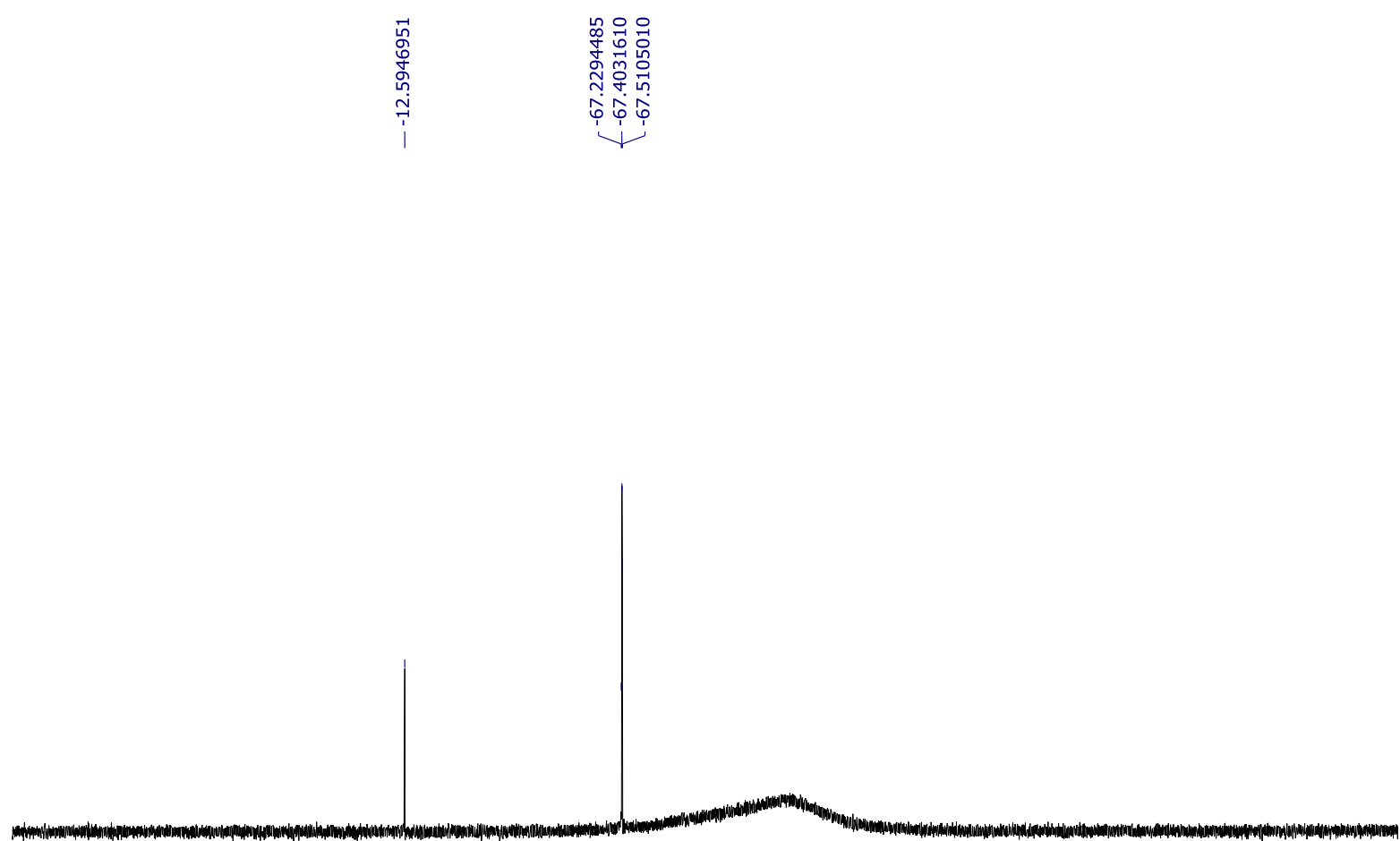

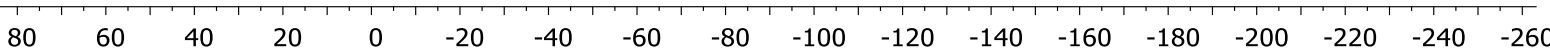


MALDI-TOF MS

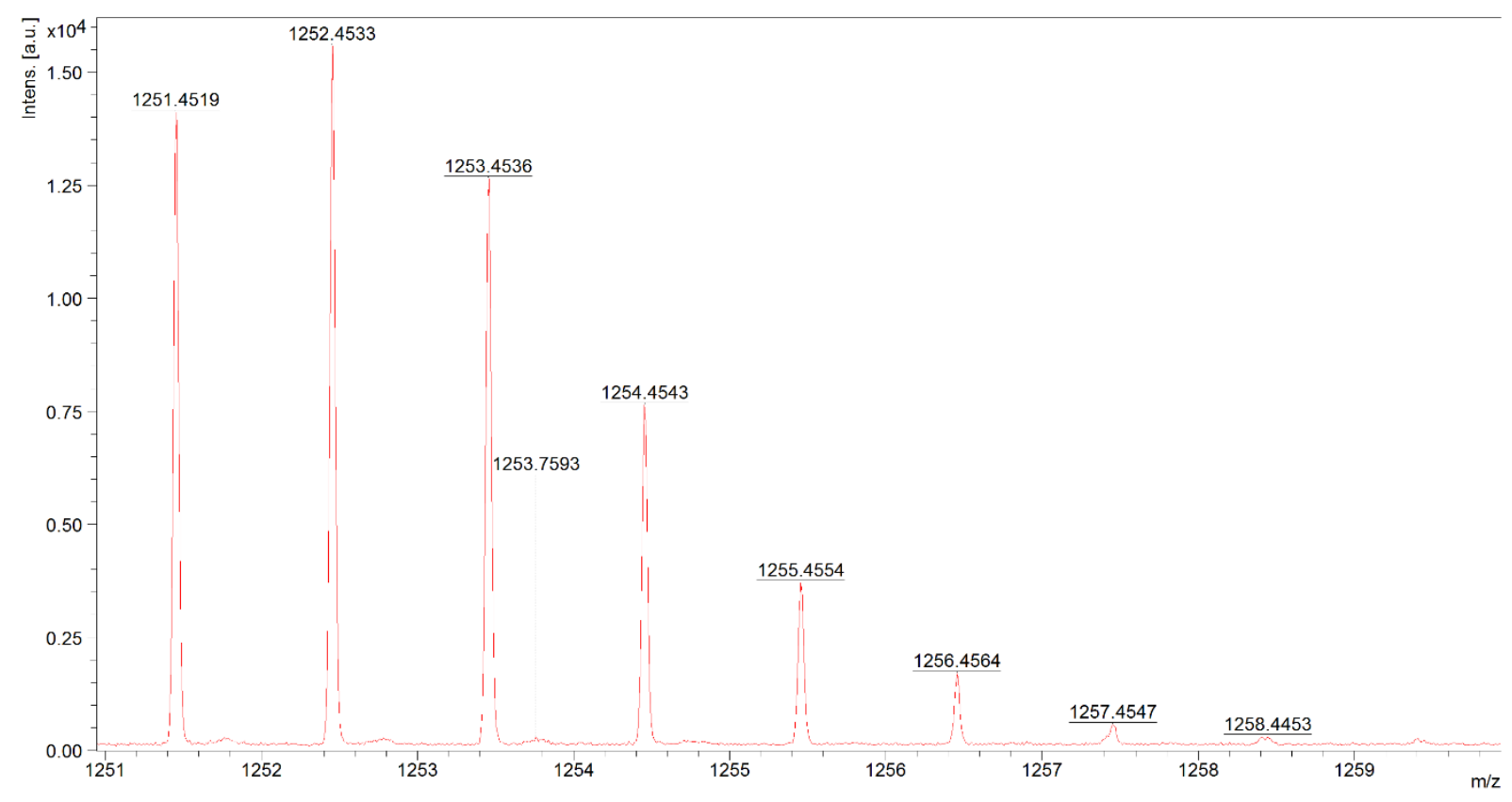


Compound 5

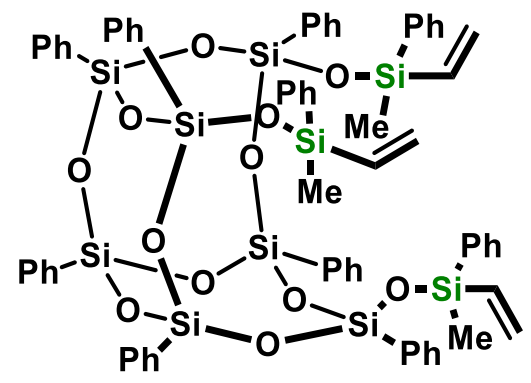

Chemical Formula: $\mathrm{C}_{69} \mathrm{H}_{68} \mathrm{O}_{12} \mathrm{Si}_{10}$

${ }^{1} \mathrm{H}$ NMR $(300 \mathrm{MHz}$, Chloroform-d) $\delta 7.79-7.28(\mathrm{~m}, 35 \mathrm{H}), 7.23-6.95(\mathrm{~m}, 15 \mathrm{H}), 6.29(\mathrm{dd}, J=20.0,15.0$ $\mathrm{Hz}, 3 \mathrm{H}), 6.09(\mathrm{dd}, J=15.0,3.9 \mathrm{~Hz}, 3 \mathrm{H}), 5.86(\mathrm{ddd}, J=20.2,16.1,4.0 \mathrm{~Hz}, 3 \mathrm{H}), 0.60-0.30(\mathrm{~m}, 9 \mathrm{H})$.

${ }^{13} \mathrm{C}$ NMR $\left(75 \mathrm{MHz}, \mathrm{CDCl}_{3}\right) \delta 137.27,136.67,134.18,133.97,132.48,131.11,130.41,130.07,129.58$, $128.01,127.80,127.60,-0.84$.

${ }^{29} \mathrm{Si} \mathrm{NMR}\left(79 \mathrm{MHz}, \mathrm{CDCl}_{3}\right) \delta-10.43,-77.33,-77.37,-77.80$.

MALDI-TOF MS (m/z): calcd. for $\mathrm{C}_{69} \mathrm{H}_{68} \mathrm{O}_{12} \mathrm{Si}_{10} \mathrm{Na}$ 1391,23; found 1391,24.

${ }^{1} \mathrm{H}$ NMR (300 MHz, $\left.\mathrm{CDCl}_{3}\right)$
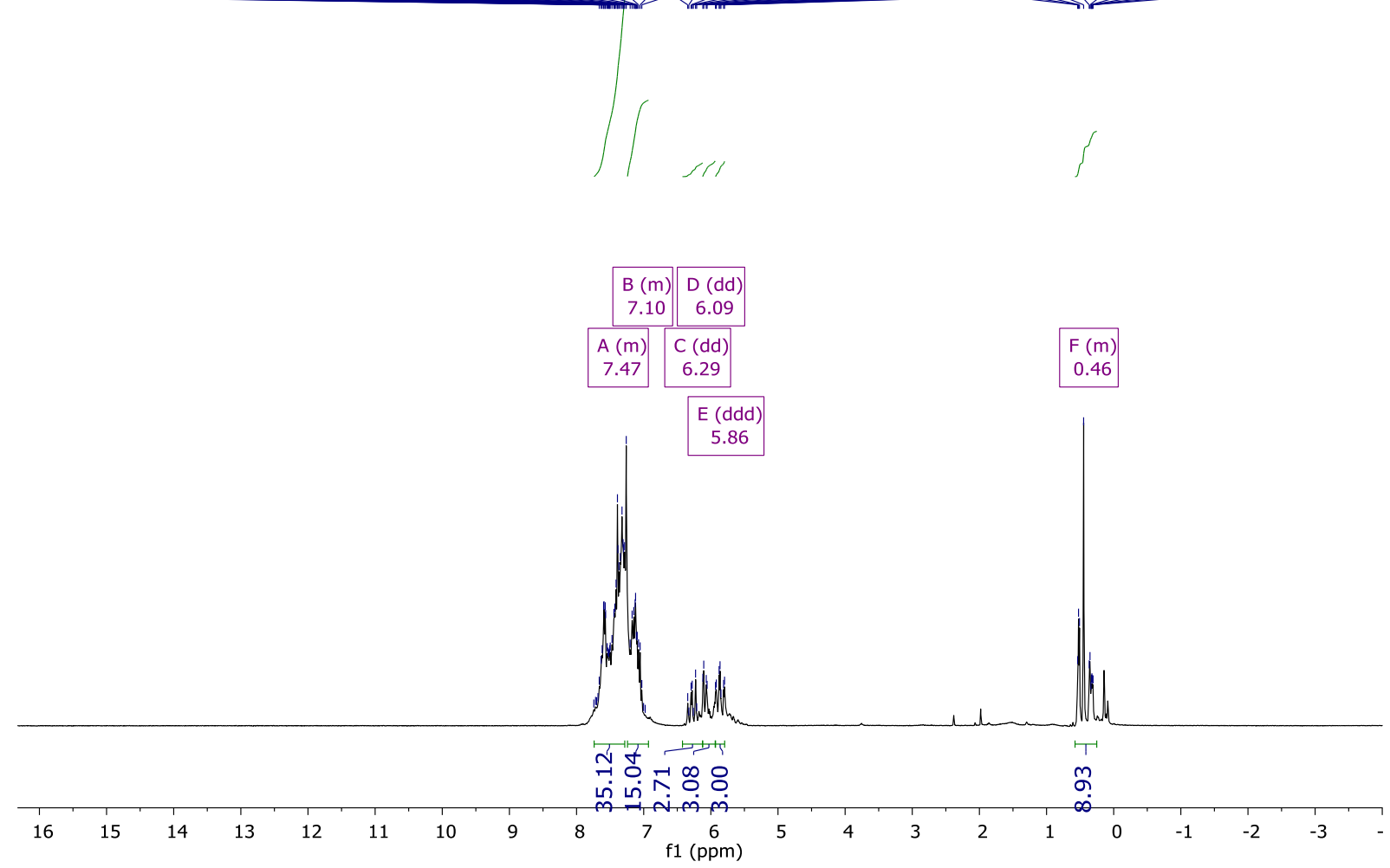
${ }^{13} \mathrm{C}$ NMR (101 MHz, $\mathrm{CDCl}_{3}$ )

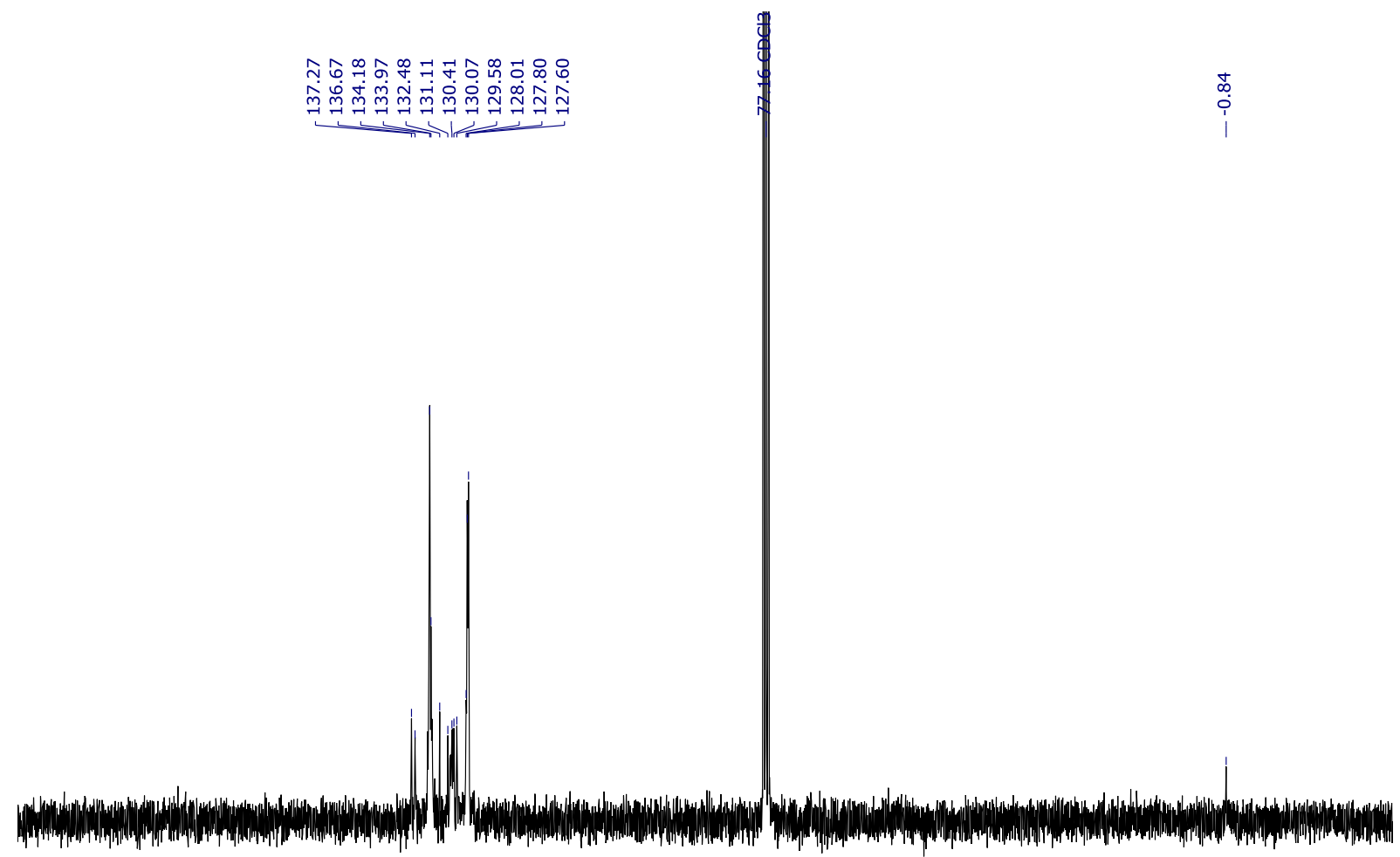

$\begin{array}{lllllllllllllllllllllll}200 & 190 & 180 & 170 & 160 & 150 & 140 & 130 & 120 & 110 & 100 & 90 & 80 & 70 & 60 & 50 & 40 & 30 & 20 & 10 & 0 & -10 & -20\end{array}$

${ }^{29} \mathrm{Si} \mathrm{NMR}\left(79 \mathrm{MHz}, \mathrm{CDCl}_{3}\right)$
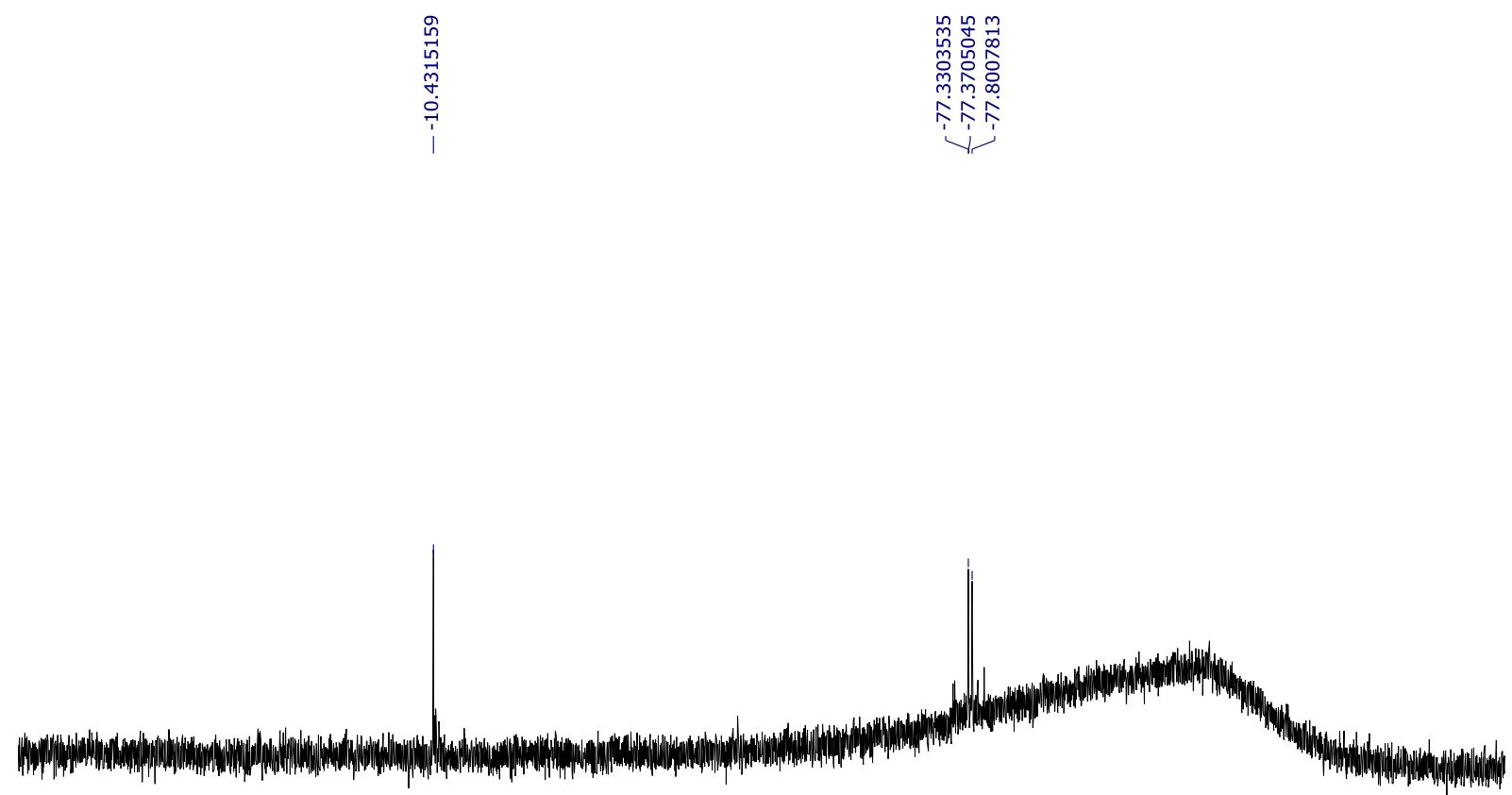

\begin{tabular}{llllllllllllllllllll}
\hline 40 & 30 & 20 & 10 & 0 & -10 & -20 & -30 & -40 & -50 & -60 & -70 & -80 & -90 & -100 & -110 & -120 & -130 & -140
\end{tabular} 
MALDI-TOF MS

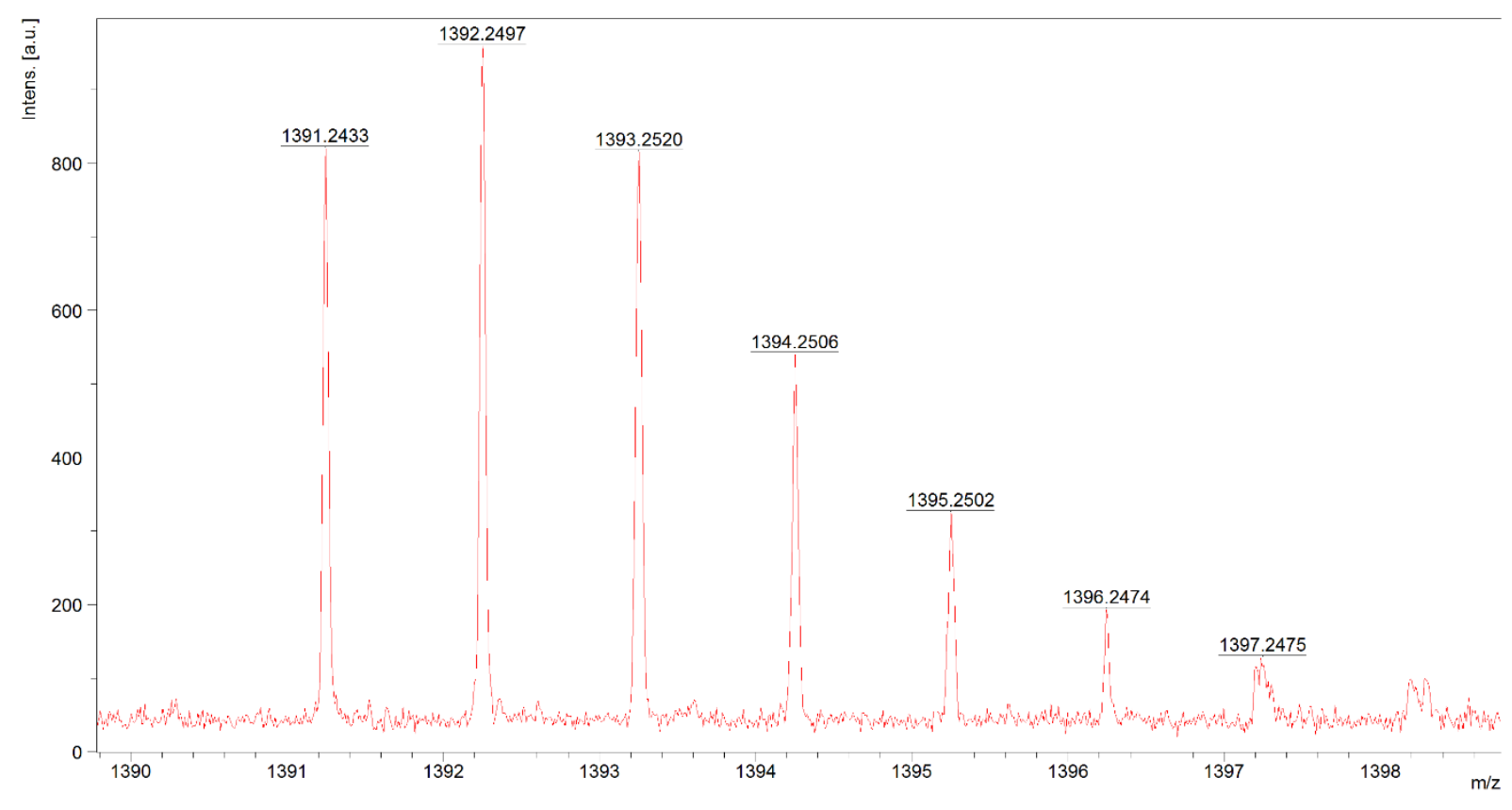


Compound 6

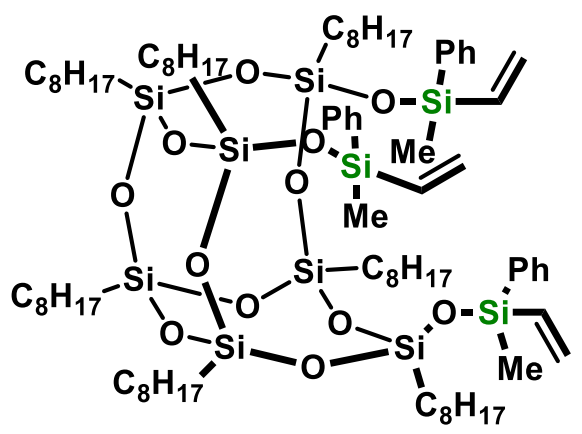

Chemical Formula: $\mathrm{C}_{83} \mathrm{H}_{152} \mathrm{O}_{12} \mathrm{Si}_{10}$

${ }^{1} \mathrm{H}$ NMR (300 MHz, Chloroform-d) $\delta 7.63-7.47(\mathrm{~m}, 6 \mathrm{H}), 7.34-7.27(\mathrm{~m}, 9 \mathrm{H}), 6.34-6.08(\mathrm{~m}, 3 \mathrm{H}), 6.04$ $-5.88(\mathrm{~m}, 3 \mathrm{H}), 5.85-5.68(\mathrm{~m}, 3 \mathrm{H}), 1.93-1.67(\mathrm{~m}, 7 \mathrm{H}), 1.35-1.08(\mathrm{~m}, 15 \mathrm{H}), 1.05-0.73(\mathrm{~m}, 90 \mathrm{H}), 0.62$ $-0.32(\mathrm{~m}, 16 \mathrm{H})$.

${ }^{13} \mathrm{C} \mathrm{NMR}\left(75 \mathrm{MHz}_{\mathrm{CDCl}}\right)$ ) $\delta$ 137.79, 137.11, 134.11, 133.90, 129.38, 127.70, 55.24, 31.32, 30.40, 26.49, $25.80,25.48,25.31,-0.68$.

${ }^{29} \mathrm{Si} \mathrm{NMR}\left(79 \mathrm{MHz}, \mathrm{CDCl}_{3}\right) \delta-12.67,-67.45,-67.63,-67.85$.

MALDI-TOF MS (m/z): calcd. for $\mathrm{C}_{83} \mathrm{H}_{15} 2 \mathrm{O}_{12} \mathrm{Si}_{10} \mathrm{Na} 1643,89$; found 1643,94.

\section{${ }^{1} \mathrm{H}$ NMR $\left(300 \mathrm{MHz}, \mathrm{CDCl}_{3}\right)$}

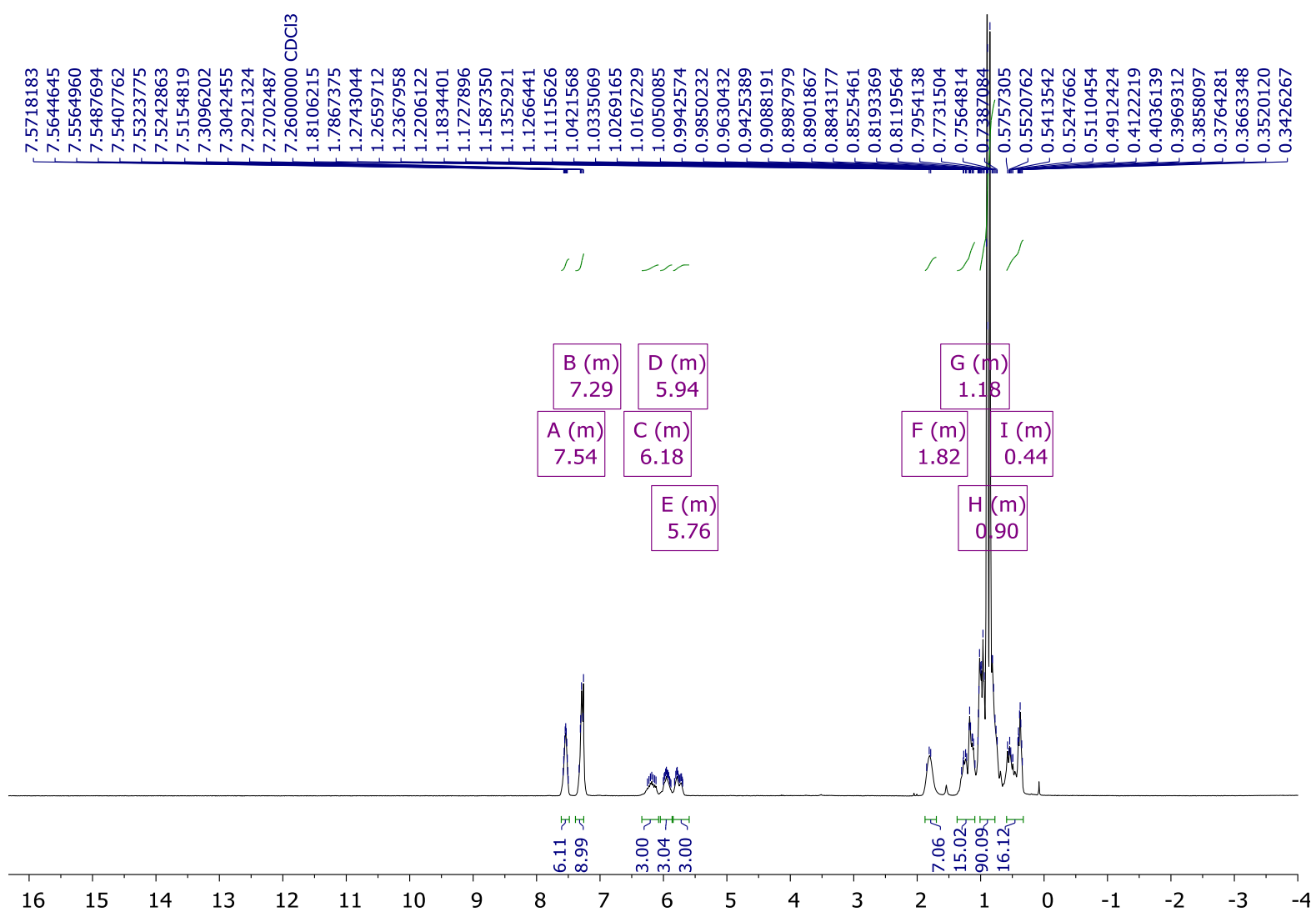


${ }^{13} \mathrm{C}$ NMR (101 MHz, $\mathrm{CDCl}_{3}$ )

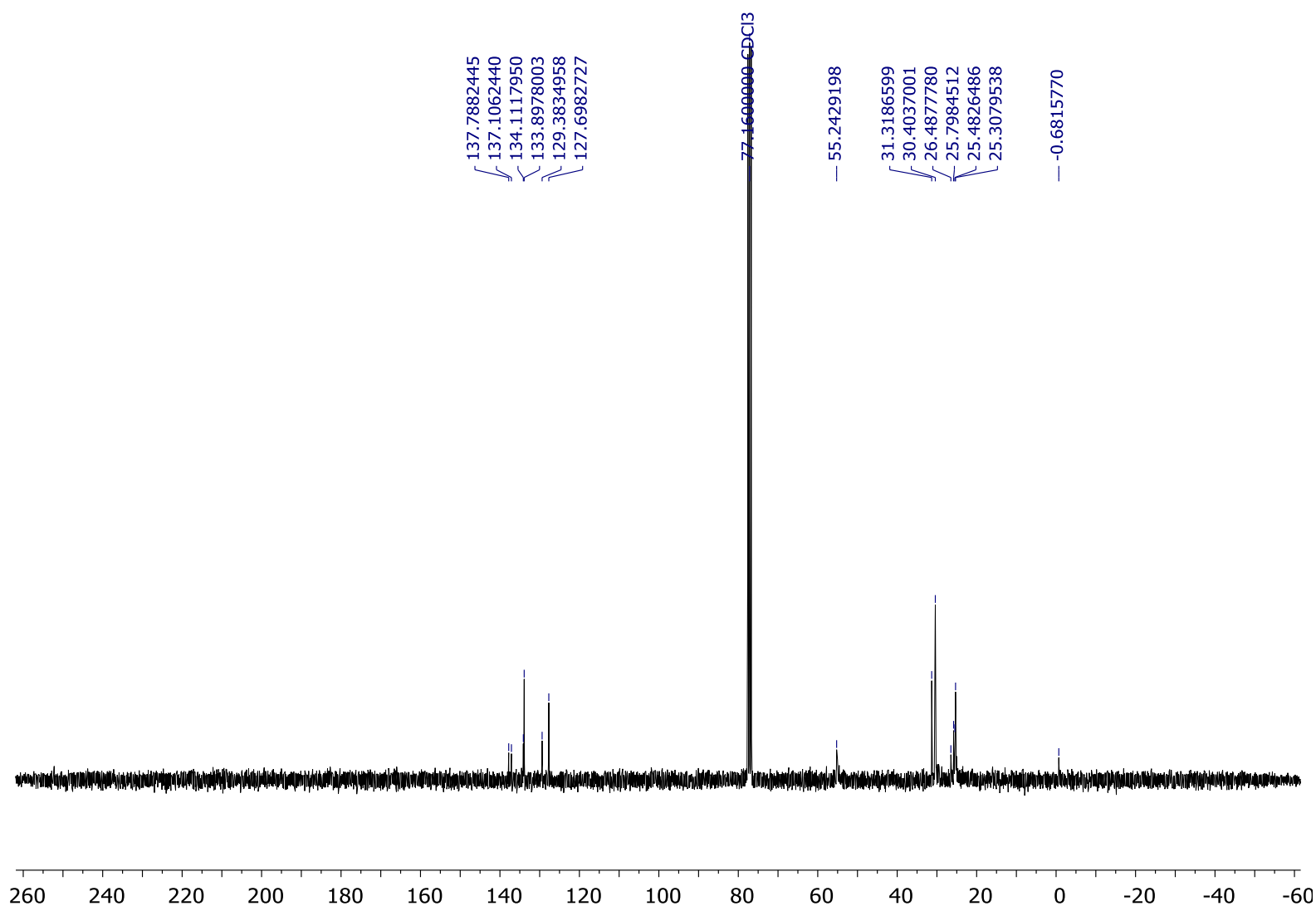

${ }^{29} \mathrm{Si} \mathrm{NMR} \mathrm{(79} \mathrm{MHz,} \mathrm{CDCl}_{3}$ )

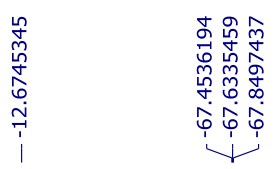

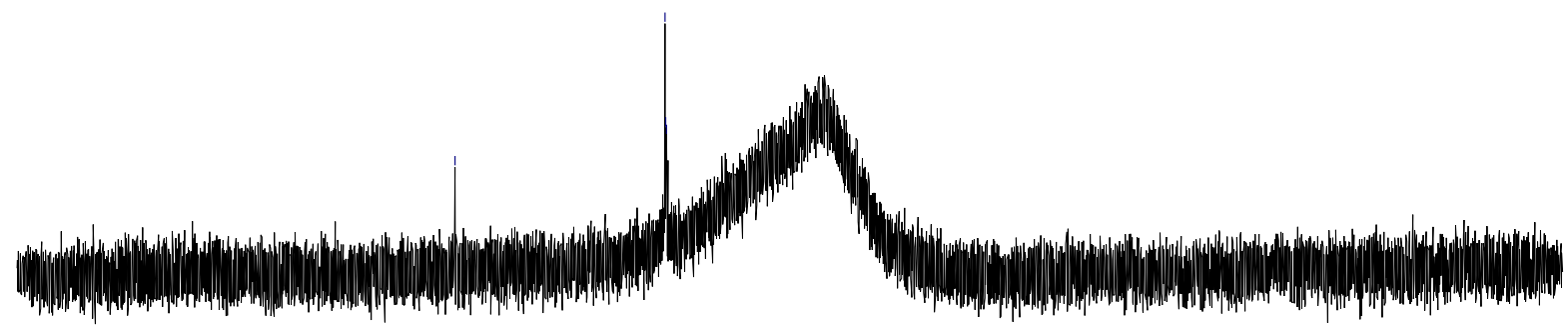

$\begin{array}{llllllllllllllllllllll}100 & 80 & 60 & 40 & 20 & 0 & -20 & -40 & -60 & -80 & -100 & -120 & -140 & -160 & -180 & -200 & -220 & -240 & -260 & -280 & -30\end{array}$ 
MALDI-TOF MS

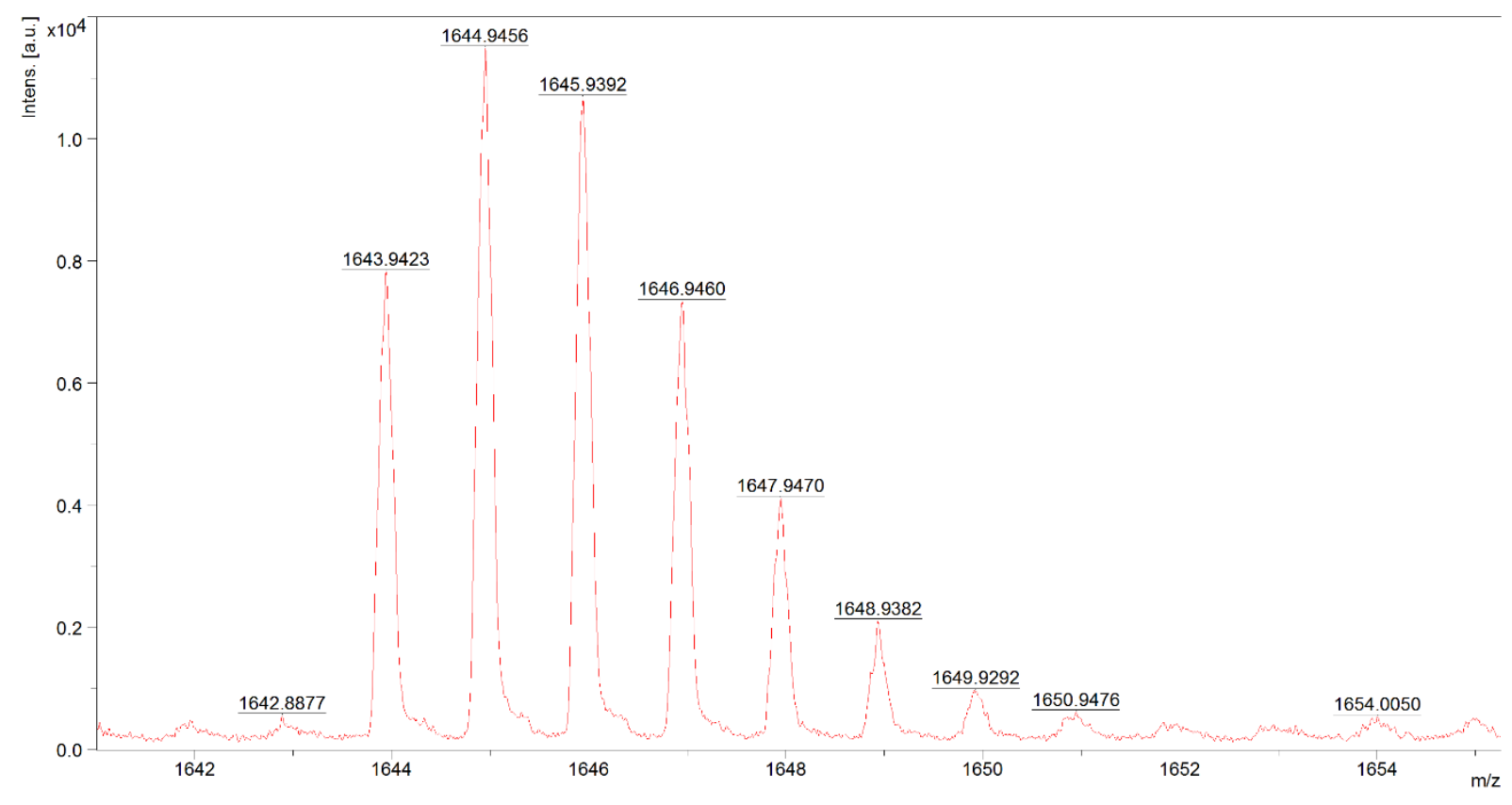


Compound 7

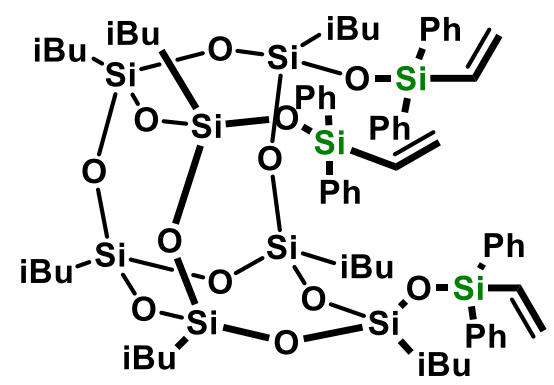

Chemical Formula: $\mathrm{C}_{70} \mathrm{H}_{102} \mathrm{O}_{12} \mathrm{Si}_{10}$

${ }^{1} \mathrm{H}$ NMR (300 MHz, Chloroform-d) $\delta 7.60-7.50(\mathrm{~m}, 12 \mathrm{H}), 7.31-7.20(\mathrm{~m}, 18 \mathrm{H}), 6.17(\mathrm{dd}, J=20.0,15.0$ $\mathrm{Hz}, 3 \mathrm{H}), 5.93-5.71(\mathrm{~m}, 6 \mathrm{H}), 1.94-1.64(\mathrm{~m}, 7 \mathrm{H}), 0.95(\mathrm{dd}, J=6.6,3.1 \mathrm{~Hz}, 24 \mathrm{H}), 0.83(\mathrm{~d}, J=6.6 \mathrm{~Hz}, 18 \mathrm{H})$, $0.53(\mathrm{dd}, J=17.4,7.0 \mathrm{~Hz}, 14 \mathrm{H}$ ).

${ }^{13} \mathrm{CNMR}\left(75 \mathrm{MHz}, \mathrm{CDCl}_{3}\right) \delta$ 136.68, 135.75, 134.87, 134.21, 129.64, 127.68, 26.12, 26.04, 25.77, 25.17, $24.06,23.98,23.87,22.59$.

${ }^{29} \mathrm{Si} \mathrm{NMR}\left(79 \mathrm{MHz}, \mathrm{CDCl}_{3}\right) \delta-22.63,-66.94,-67.26,-67.45$.

MALDI-TOF MS (m/z): calcd. for $\mathrm{C}_{70} \mathrm{H}_{102} \mathrm{O}_{12} \mathrm{Si}_{10} \mathrm{Na}$ 1437,50; found 1437,49.

${ }^{1} \mathrm{H}$ NMR (300 MHz, $\mathrm{CDCl}_{3}$ )

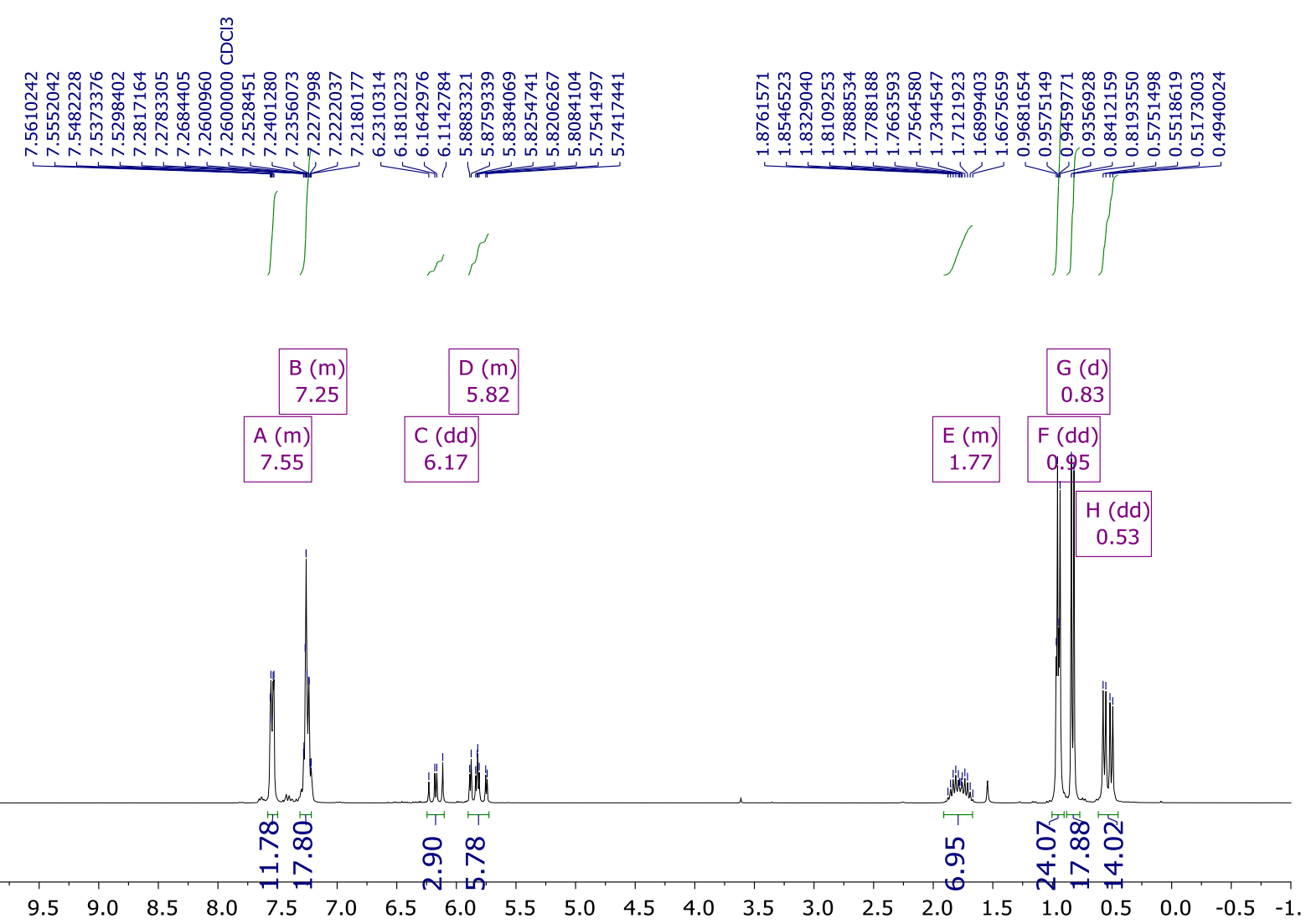


${ }^{13} \mathrm{C}$ NMR (101 MHz, $\mathrm{CDCl}_{3}$ )

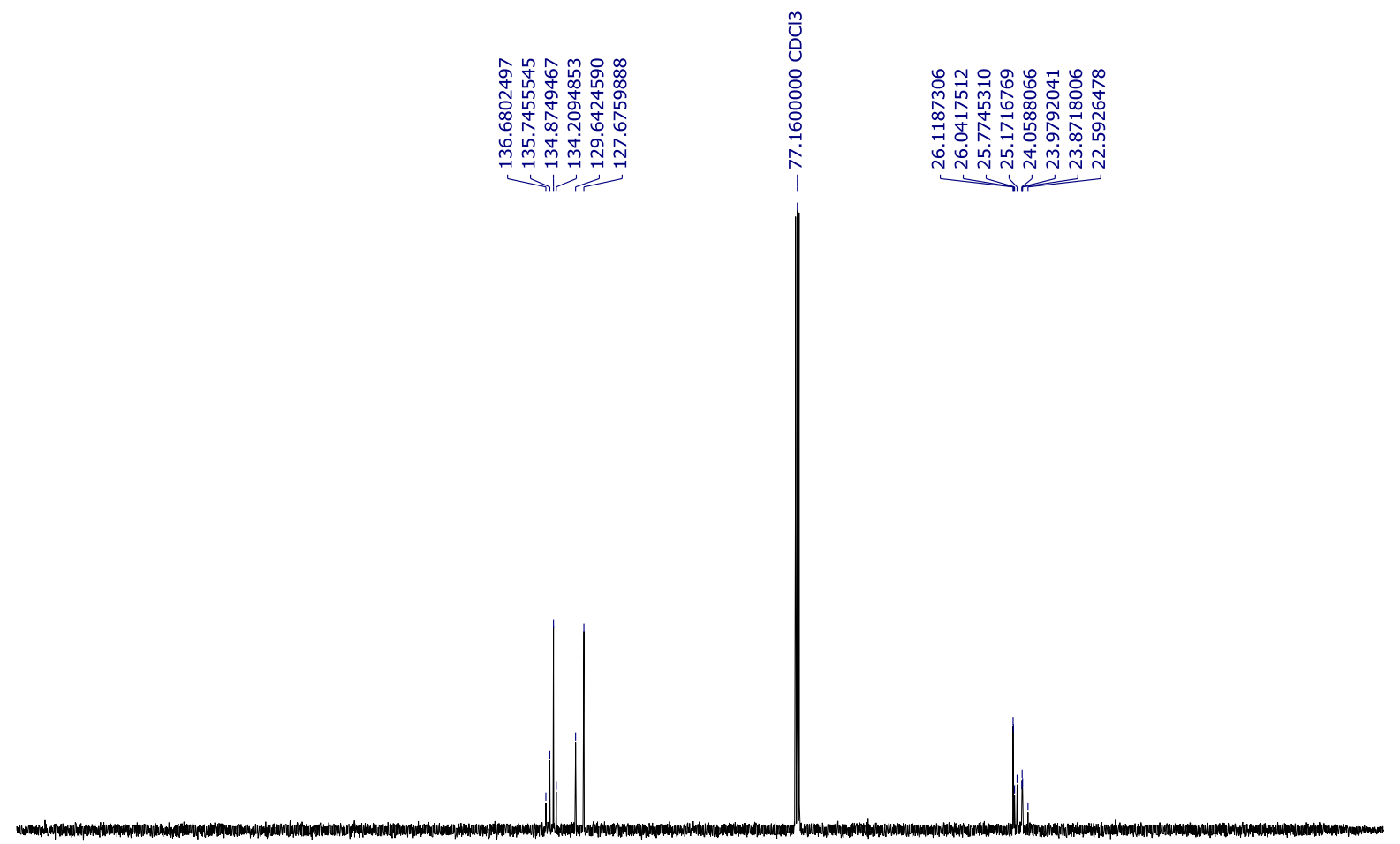

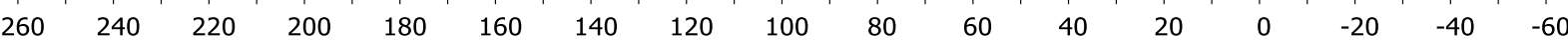

${ }^{29} \mathrm{Si} \mathrm{NMR} \mathrm{(79} \mathrm{MHz,} \mathrm{CDCl}_{3}$ )

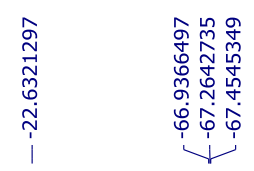

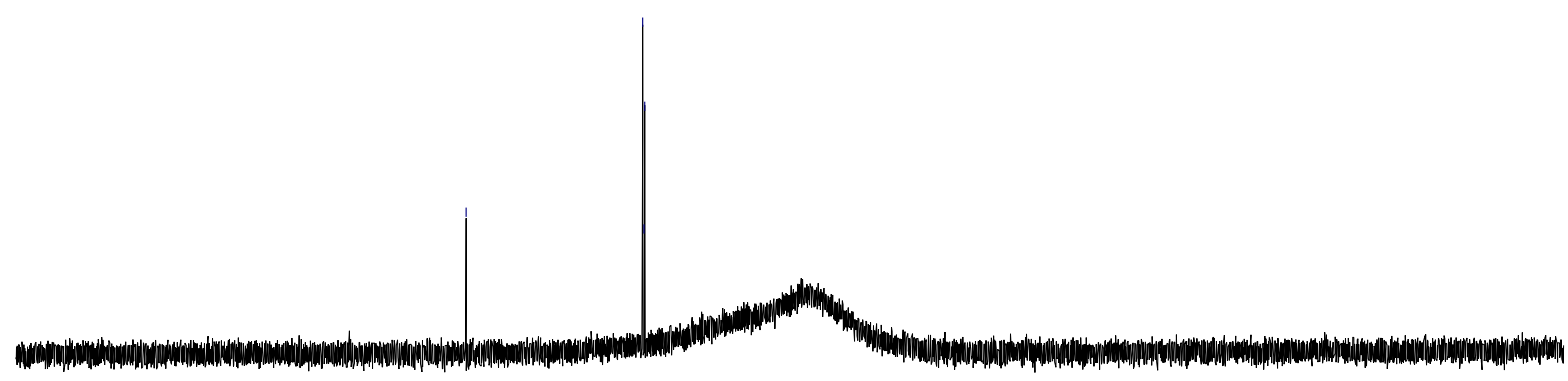

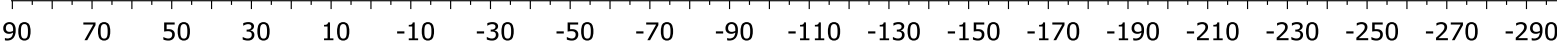


MALDI-TOF MS

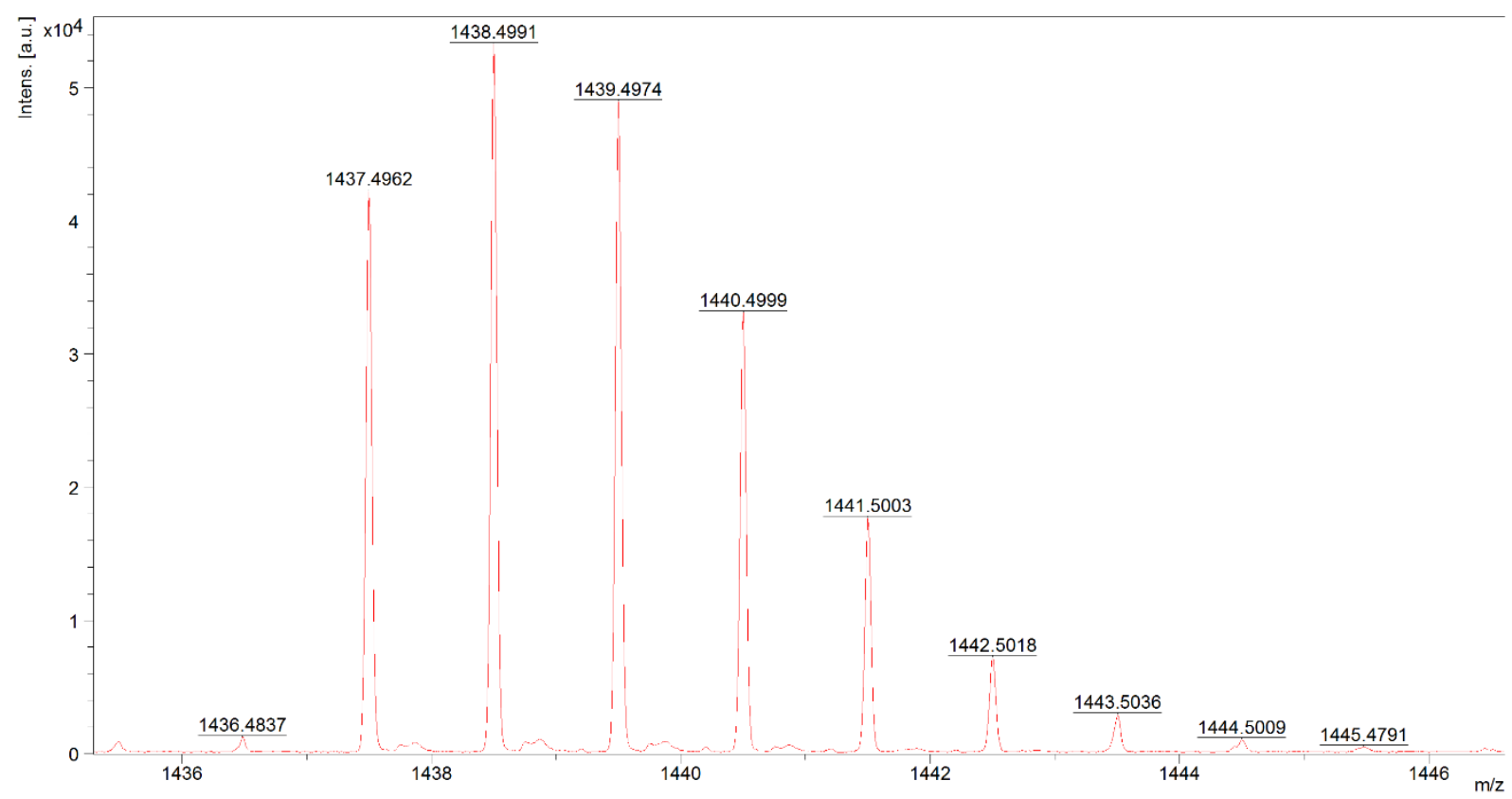


Compound 8

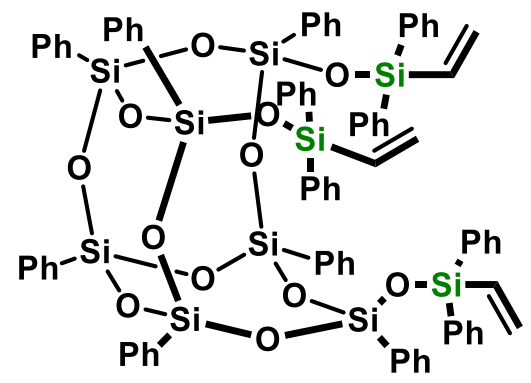

Chemical Formula: $\mathrm{C}_{84} \mathrm{H}_{74} \mathrm{O}_{12} \mathrm{Si}_{10}$

${ }^{1} \mathrm{H}$ NMR (300 MHz, Chloroform-d) $87.60-7.28(\mathrm{~m}, 32), 7.25-6.89(\mathrm{~m}, 33 \mathrm{H}), 6.38-5.50(\mathrm{~m}, 9 \mathrm{H})$.

${ }^{13} \mathrm{C}$ NMR $\left(75 \mathrm{MHz}, \mathrm{CDCl}_{3}\right) \delta 135.66,135.29,134.95,134.89,134.79,134.68,134.40,134.34,134.25$, $134.19,129.92,129.79,129.68,127.85,127.80,127.75,127.72,127.63,127.55,127.48$.

${ }^{29} \mathrm{Si}$ NMR $\left(79 \mathrm{MHz}, \mathrm{CDCl}_{3}\right) \delta-20.66,-77.08,-77.43,-77.50$.

MALDI-TOF MS (m/z): calcd. for $\mathrm{C}_{84} \mathrm{H}_{74} \mathrm{O}_{12} \mathrm{Si}_{10} \mathrm{Na}$ 1577,28; found 1577,28.

${ }^{1} \mathrm{H}$ NMR $\left(300 \mathrm{MHz}, \mathrm{CDCl}_{3}\right)$

莙

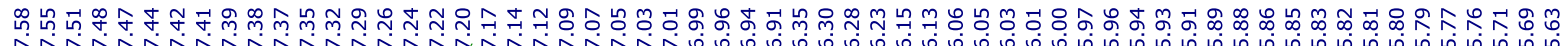

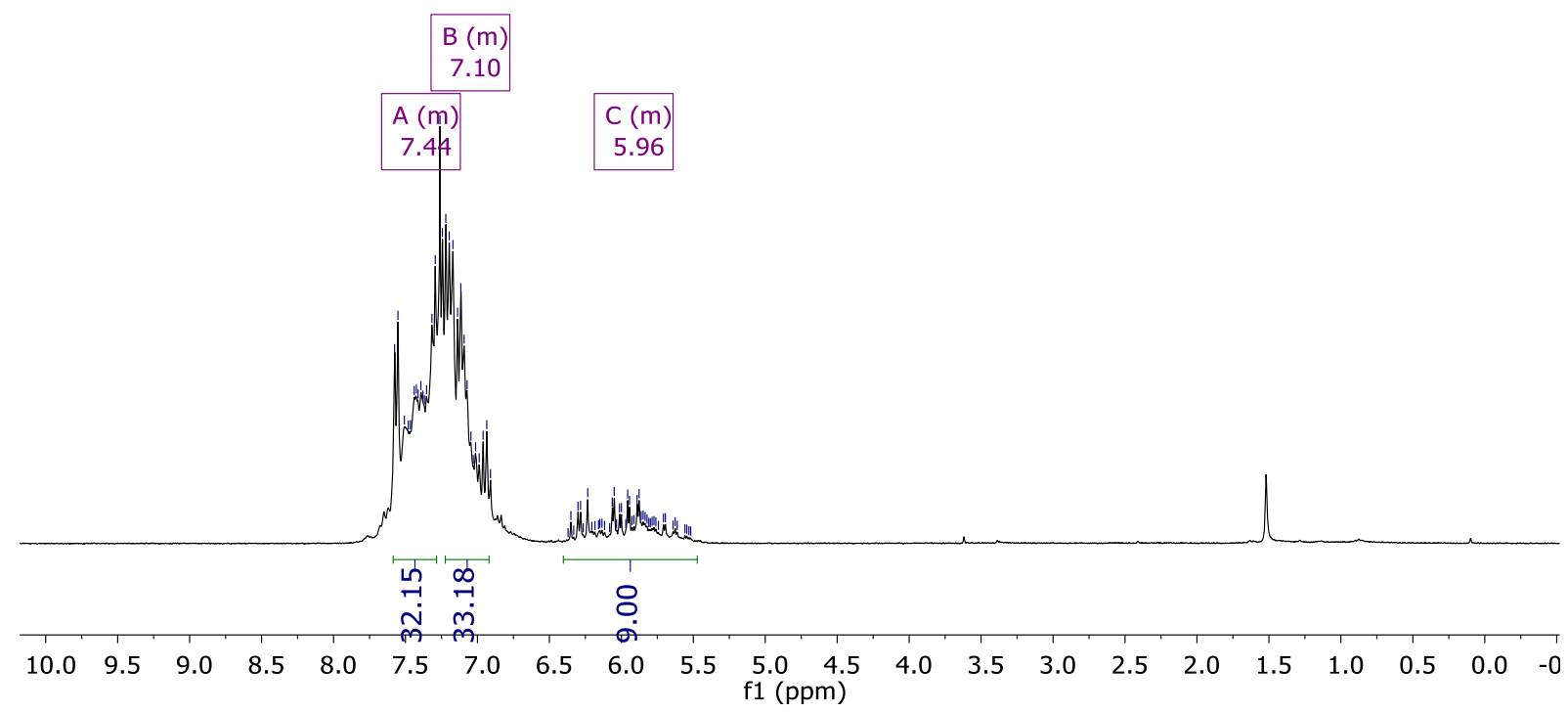


$\left.{ }^{13} \mathrm{C} \mathrm{NMR} \mathrm{(101} \mathrm{MHz,} \mathrm{CDCl}_{3}\right)$

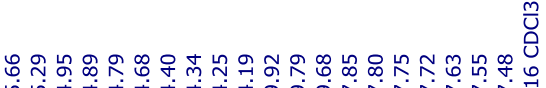

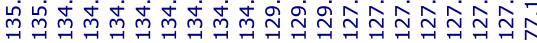

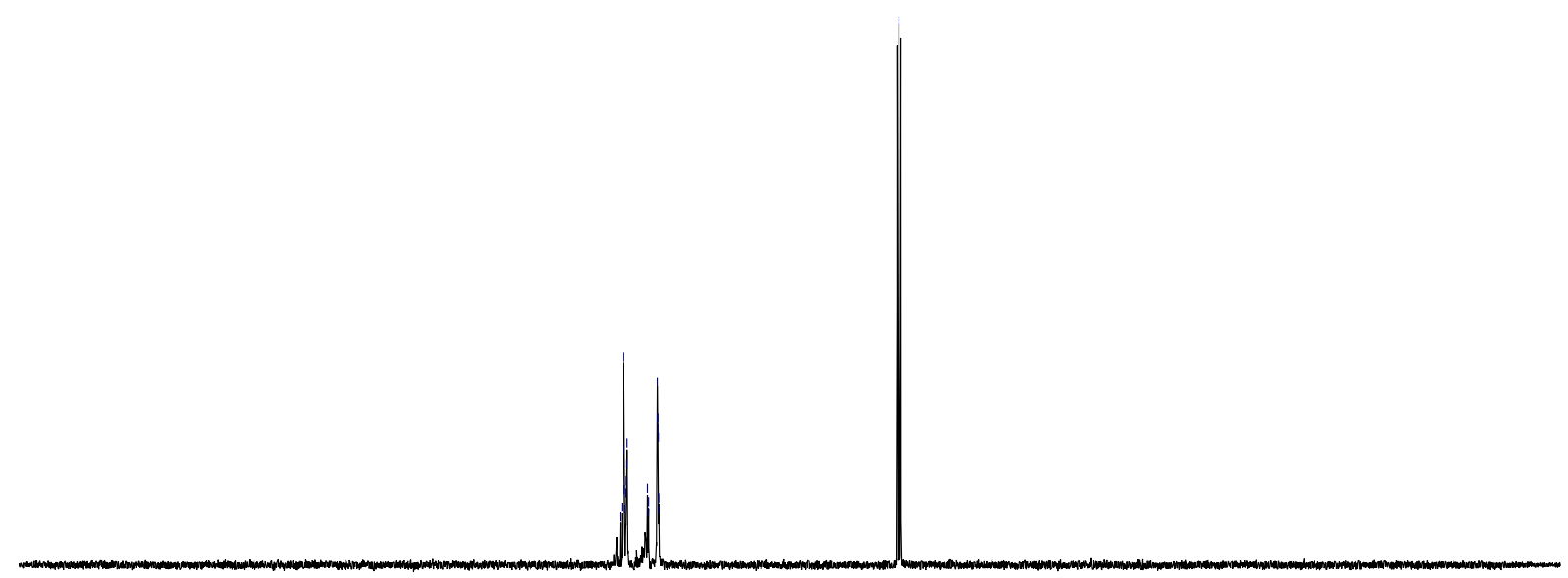

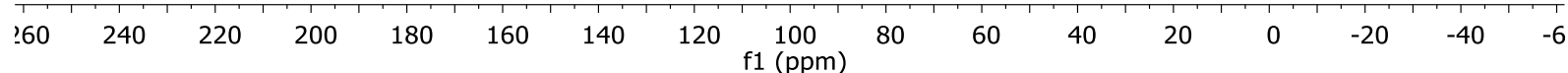

${ }^{29} \mathrm{Si} \mathrm{NMR}\left(79 \mathrm{MHz}, \mathrm{CDCl}_{3}\right)$

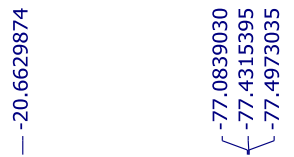

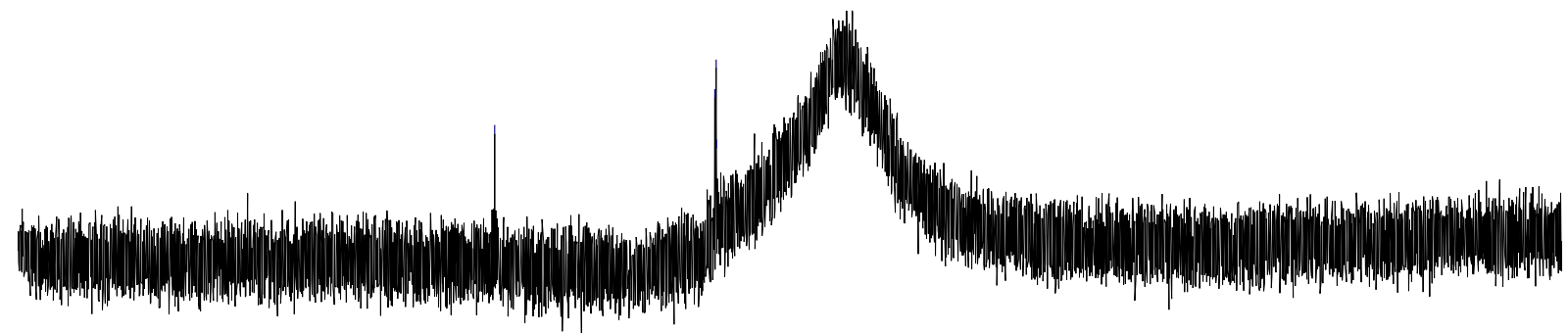

$\begin{array}{llllllllllllllllllll}90 & 70 & 50 & 30 & 10 & -10 & -30 & -50 & -70 & -90 & -110 & -130 & -150 & -170 & -190 & -210 & -230 & -250 & -270 & -29\end{array}$ 
MALDI-TOF MS

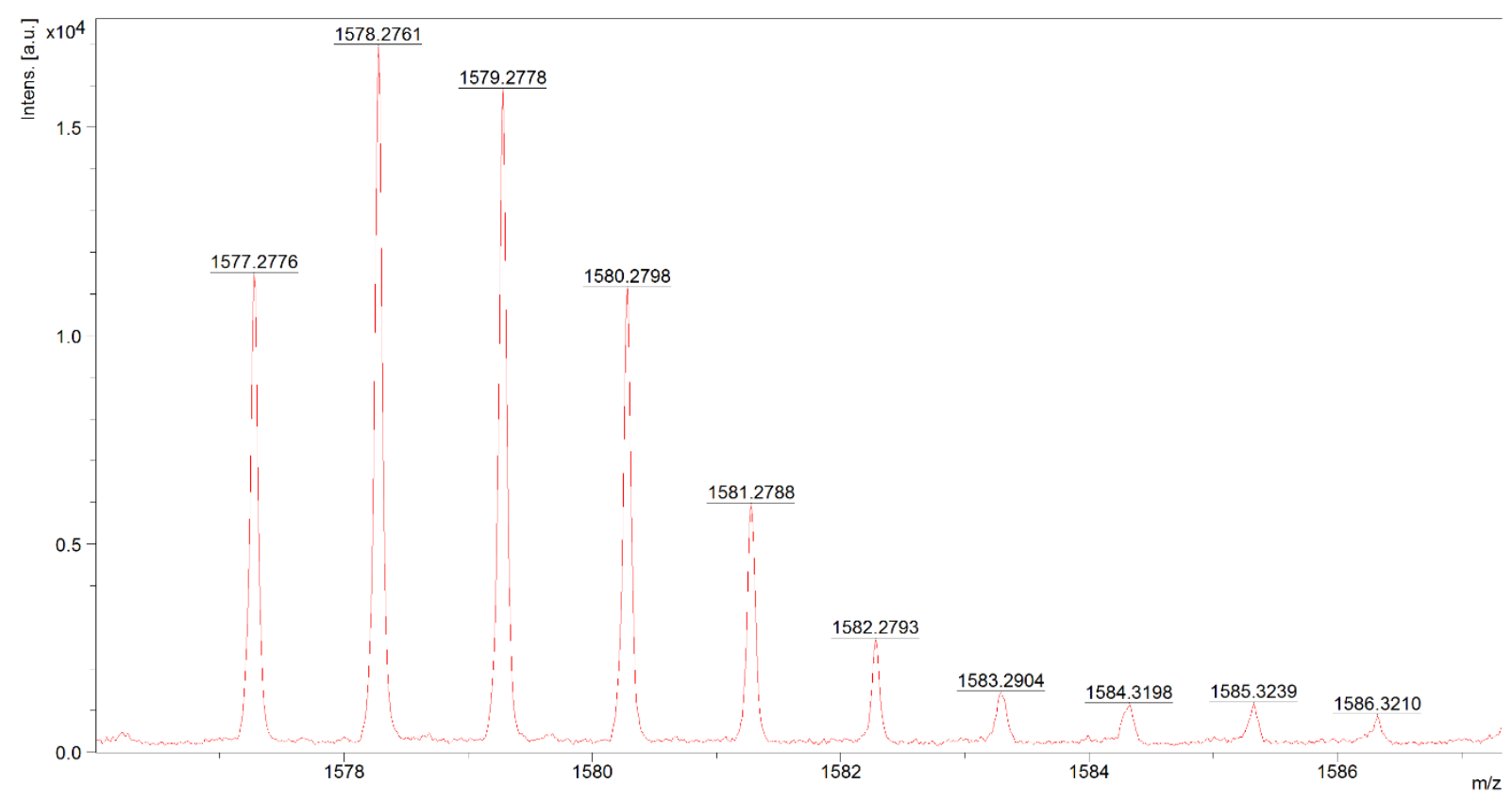


Compound 9

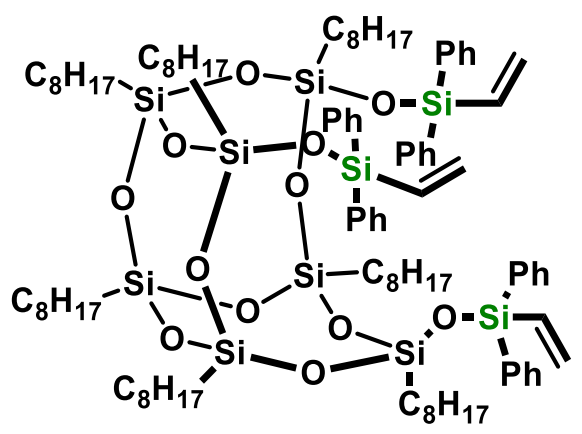

Chemical Formula: $\mathrm{C}_{98} \mathrm{H}_{158} \mathrm{O}_{12} \mathrm{Si}_{10}$

${ }^{1} \mathrm{H}$ NMR (300 MHz, Chloroform-d) $\delta 7.68-7.52(\mathrm{~m}, 12 \mathrm{H}), 7.49-7.28(\mathrm{~m}, 18 \mathrm{H}), 6.51(\mathrm{dd}, J=20.2,14.9$ $\mathrm{Hz}, 3 \mathrm{H}), 6.27(\mathrm{dd}, J=14.8,3.8 \mathrm{~Hz}, 3 \mathrm{H}), 5.95(\mathrm{dd}, J=20.2,3.8 \mathrm{~Hz}, 3 \mathrm{H}), 1.92-1.69(\mathrm{~m}, 7 \mathrm{H}), 1.31(\mathrm{dd}, J=$ 13.9, $4.5 \mathrm{~Hz}, 5 \mathrm{H}), 1.23-1.08(\mathrm{~m}, 7 \mathrm{H}), 1.06-0.69(\mathrm{~m}, 93 \mathrm{H}), 0.65-0.44(\mathrm{~m}, 7 \mathrm{H})$.

${ }^{13} \mathrm{C} \mathrm{NMR}\left(75 \mathrm{MHz}, \mathrm{CDCl}_{3}\right)$ ) 134.93, 134.83, 129.79, 129.63, 127.82, 127.69, 55.20, 31.31, 31.22, 31.20, $30.35,25.74,25.72,25.68,25.63,25.58,25.56,25.53,25.45,25.39,25.33,25.31,25.30,25.24,25.21$, $25.17,25.09,25.07,25.01,0.05$.

${ }^{29} \mathrm{Si} \mathrm{NMR}\left(79 \mathrm{MHz}, \mathrm{CDCl}_{3}\right)$ $\delta$-21.99, -22.35, -22.40, -66.72, -66.82, -67.20, -67.28, -67.44, -68.21, -68.55, $-68.61,-68.65$.

MALDI-TOF MS (m/z): calcd. for $\mathrm{C}_{98} \mathrm{H}_{158} \mathrm{O}_{12} \mathrm{Si}_{10} \mathrm{Na}$ 1829,93; found 1829,99.

\section{${ }^{1} \mathrm{H}$ NMR (300 MHz, $\mathrm{CDCl}_{3}$ )}

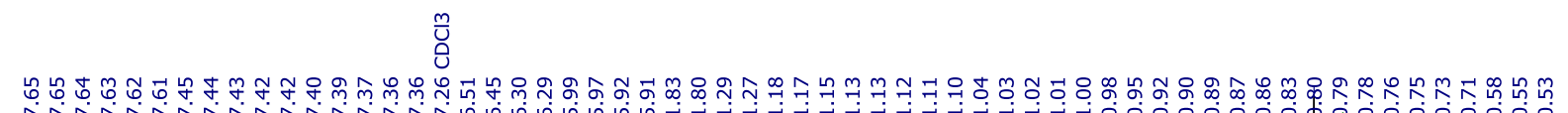

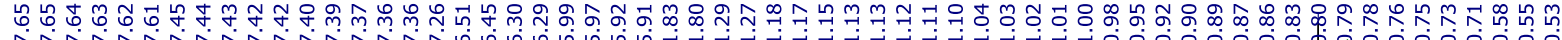

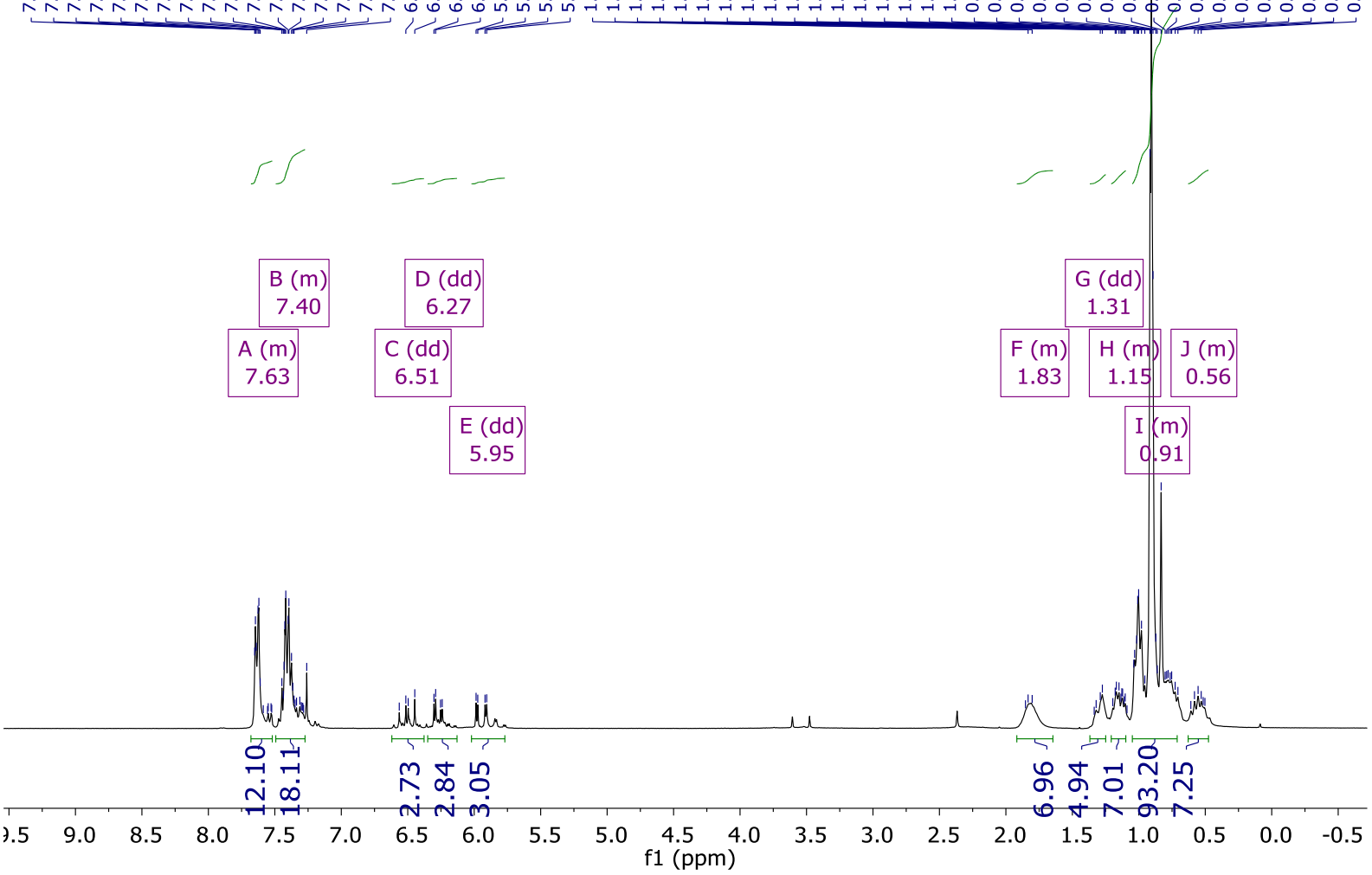


${ }^{13} \mathrm{C}$ NMR (101 MHz, $\mathrm{CDCl}_{3}$ )

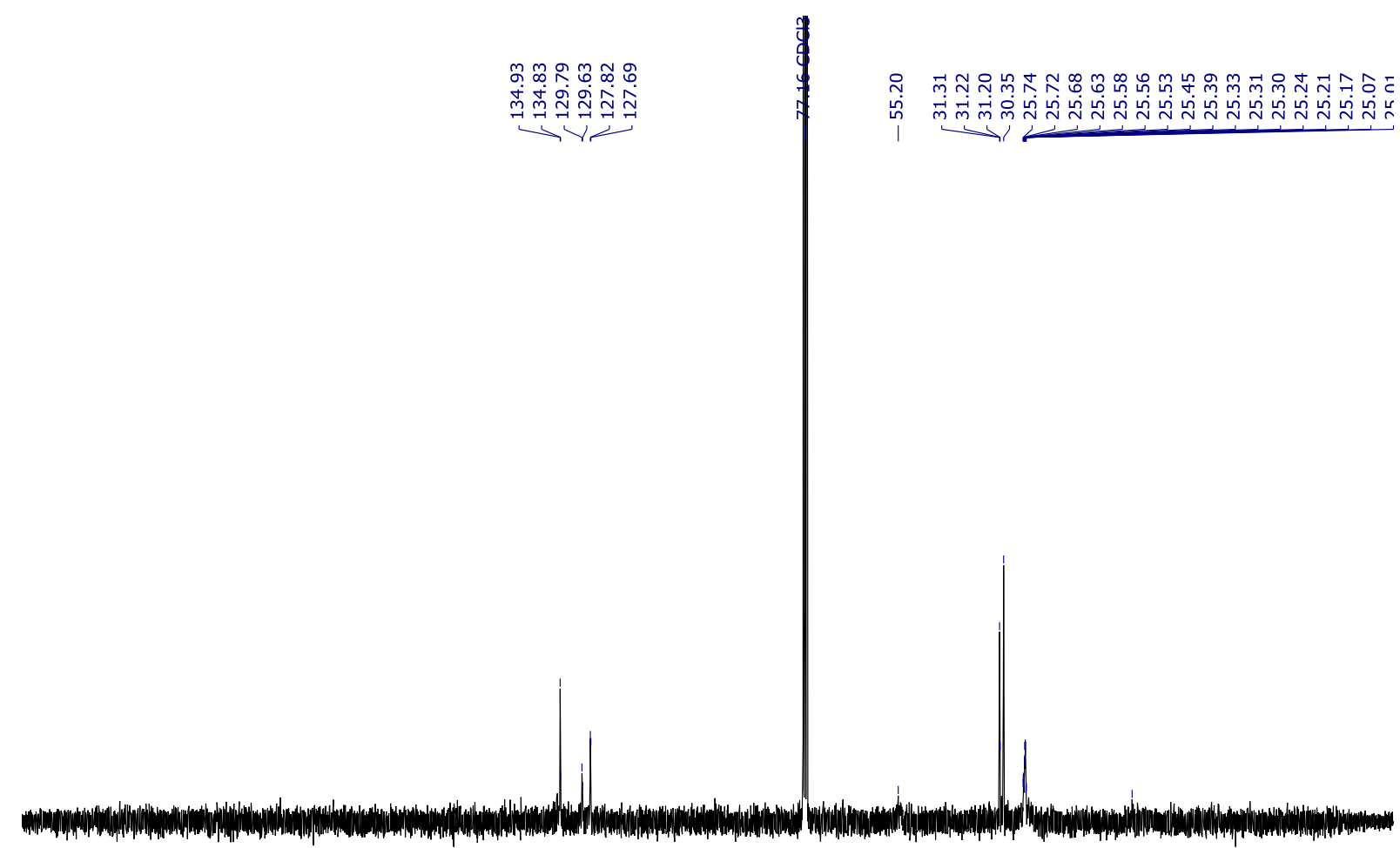

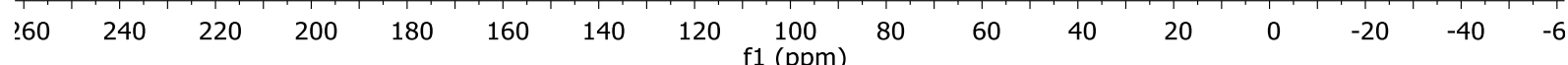

${ }^{29}$ Si NMR (79 MHz, $\left.\mathrm{CDCl}_{3}\right)$
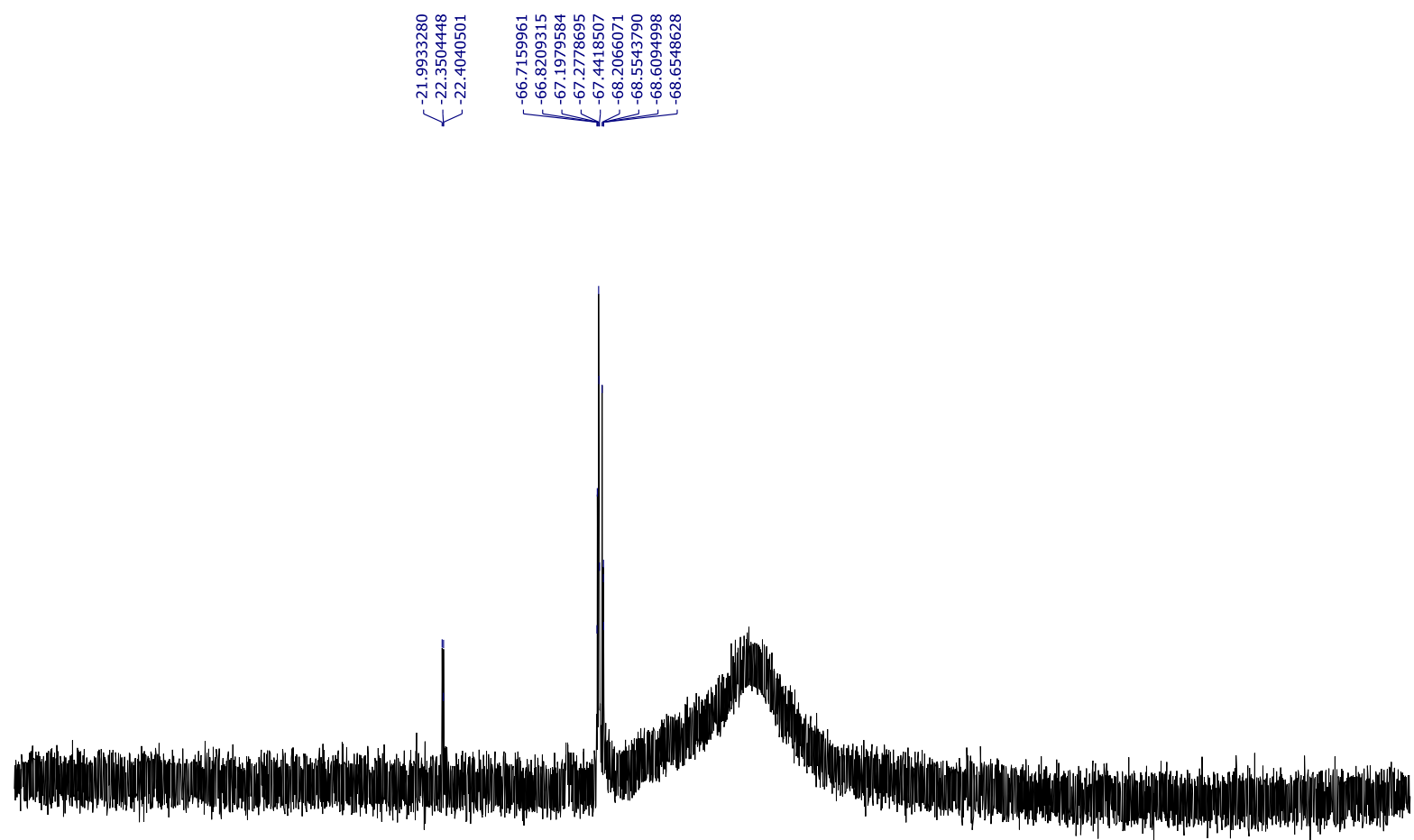

$\begin{array}{llllllllllllllllllllllllll}100 & 80 & 60 & 40 & 20 & 0 & -20 & -40 & -60 & -80 & -100 & -120 & -140 & -160 & -180 & -200 & -220 & -240 & -260 & -280 & -30\end{array}$ 
MALDI-TOF MS

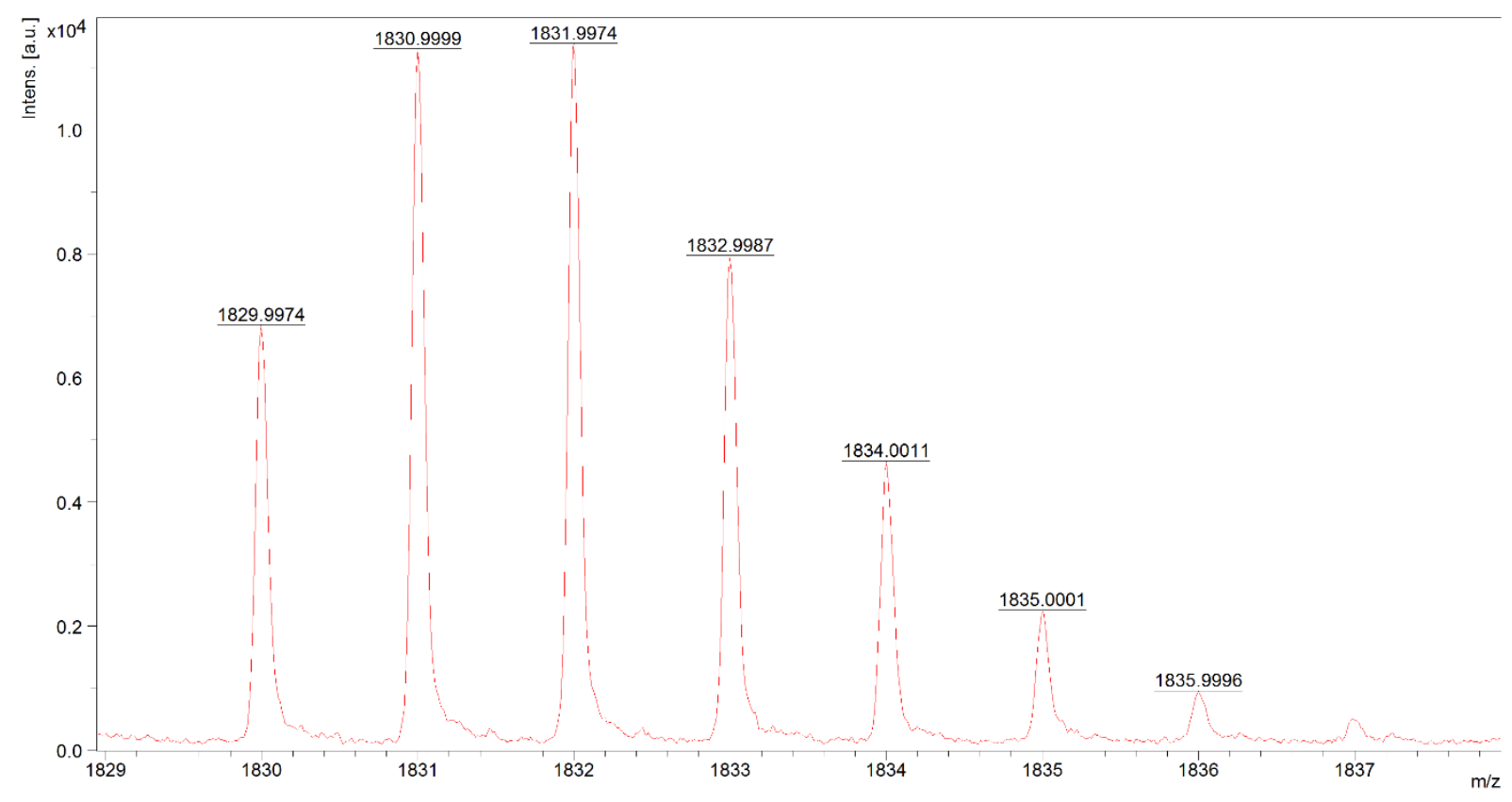




\section{The ${ }^{1} \mathrm{H}$ NMR, ${ }^{13} \mathrm{C}$ NMR, ${ }^{29} \mathrm{Si}$ NMR, and MALDI TOF of functionalized IC-POSS}

Compound $1 \mathrm{a}$

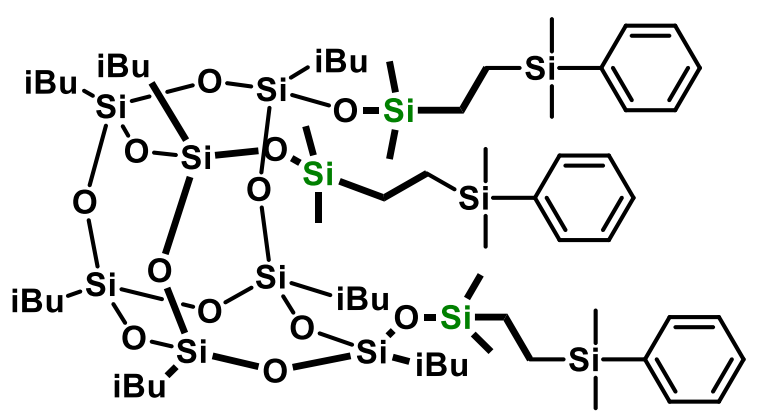

Chemical Formula: $\mathrm{C}_{64} \mathrm{H}_{126} \mathrm{O}_{12} \mathrm{Si}_{13}$

${ }^{1} \mathrm{H}$ NMR (300 MHz, Chloroform-d) $\delta 7.58-7.47(\mathrm{~m}, 6 \mathrm{H}), 7.40-7.31(\mathrm{~m}, 9 \mathrm{H}), 1.95-1.76(\mathrm{~m}, 7 \mathrm{H}), 0.98$ (dd, $J=6.6,2.6 \mathrm{~Hz}, 42 \mathrm{H}), 0.76-0.64(\mathrm{~m}, 6 \mathrm{H}), 0.57(\mathrm{dd}, J=7.1,3.0 \mathrm{~Hz}, 14 \mathrm{H}), 0.53-0.44(\mathrm{~m}, 6 \mathrm{H}), 0.27$ $(\mathrm{s}, 18 \mathrm{H}), 0.12(\mathrm{~s}, 18 \mathrm{H})$.

${ }^{13} \mathrm{C} \mathrm{NMR}\left(75 \mathrm{MHz}, \mathrm{CDCl}_{3}\right) \delta$ 139.72, 133.77, 128.86, 127.82, 26.20, 26.02, 25.79, 25.22, 24.26, 24.15, $24.02,23.93,22.62,10.21,7.18,-0.22,-3.40,-3.43$.

${ }^{29} \mathrm{Si} \mathrm{NMR}\left(79 \mathrm{MHz}, \mathrm{CDCl}_{3}\right) \delta 9.67,-1.36,-67.30,-67.80,-68.16$.

MALDI-TOF MS (m/z): calcd. for $\mathrm{C}_{64} \mathrm{H}_{126} \mathrm{O}_{12} \mathrm{Si}_{13} \mathrm{Na}$ 1475,79; found 1475,61.

\section{${ }^{1} \mathrm{H}$ NMR $\left(300 \mathrm{MHz}, \mathrm{CDCl}_{3}\right)$}

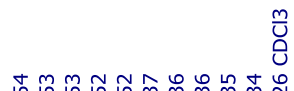

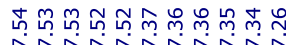

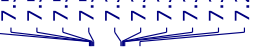

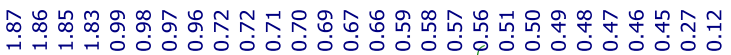
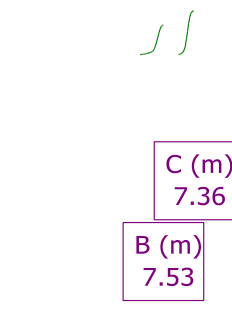

\begin{tabular}{c}
\hline $\begin{array}{c}C(\mathrm{~m}) \\
7.36\end{array}$ \\
$B(\mathrm{~m})$ \\
7.53 \\
\hline
\end{tabular}
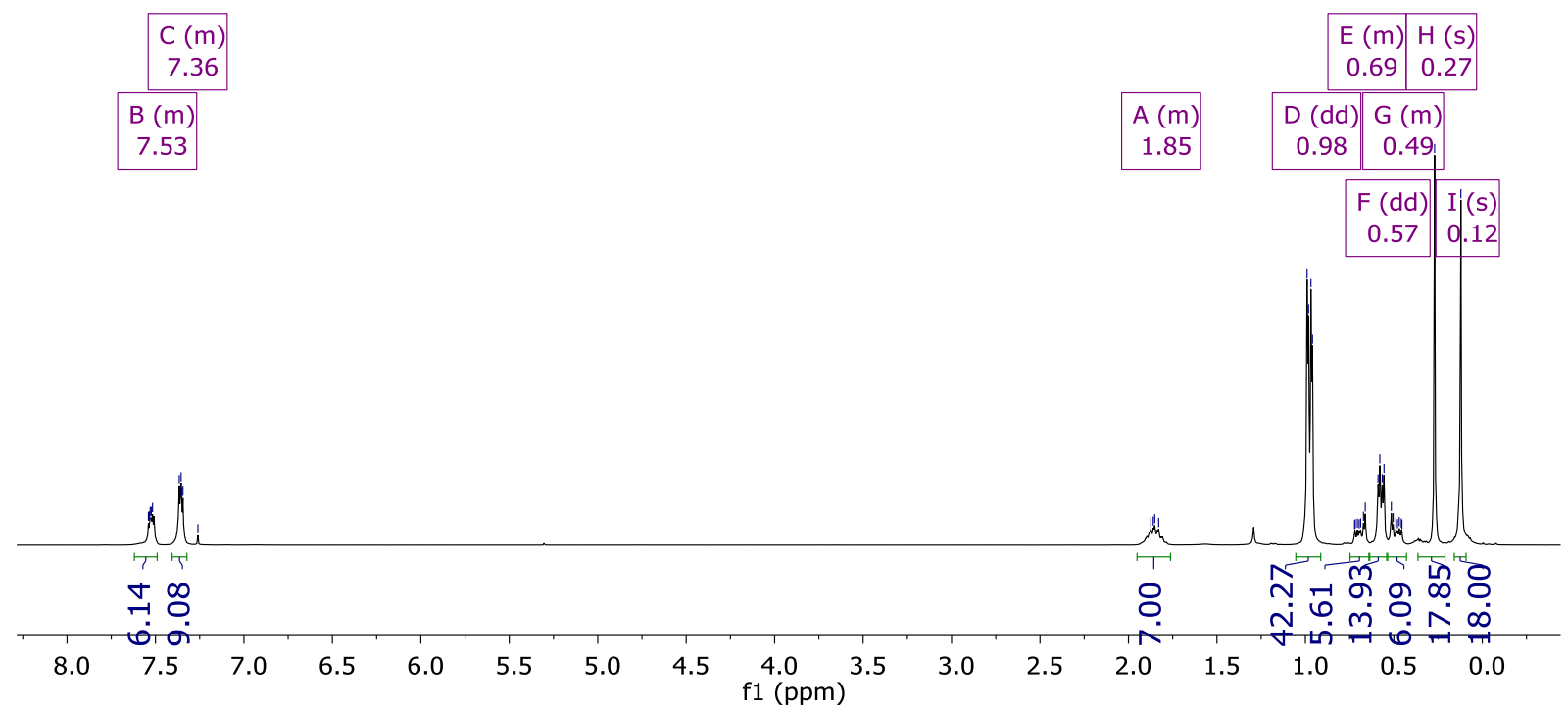
${ }^{13} \mathrm{C}$ NMR (101 MHz, $\mathrm{CDCl}_{3}$ )

م्ల్య

$\frac{m}{\mathrm{U}}$

눈

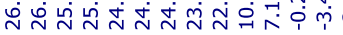

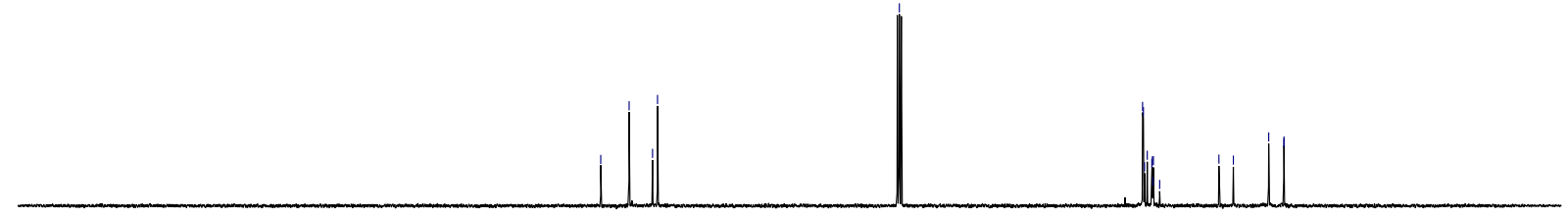

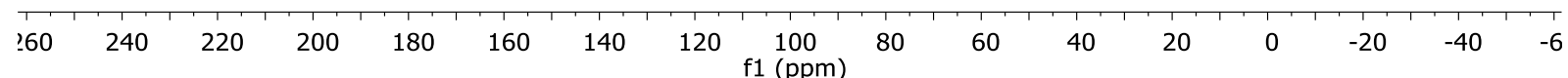

${ }^{29}$ Si NMR (79 MHz, $\left.\mathrm{CDCl}_{3}\right)$
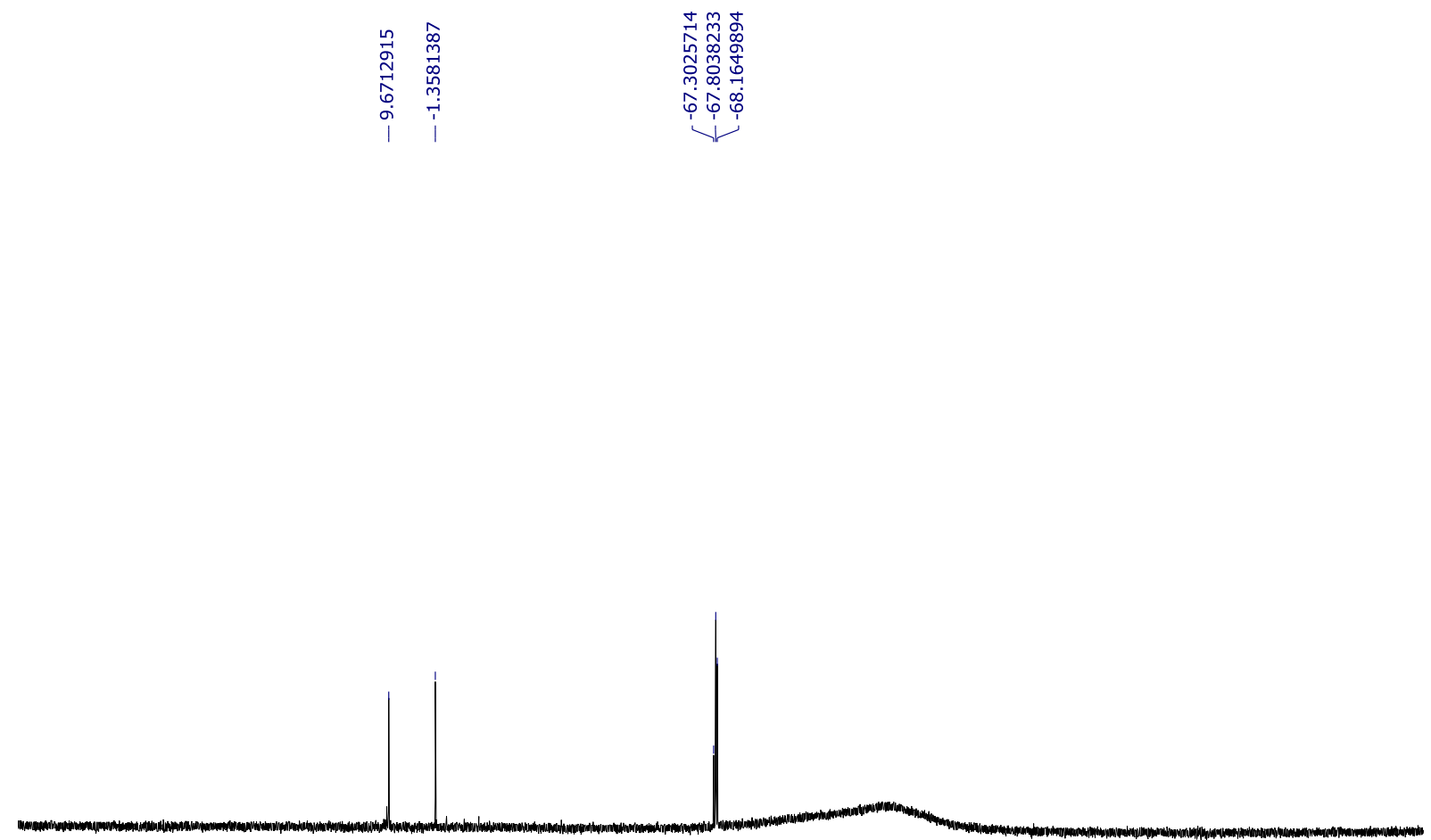

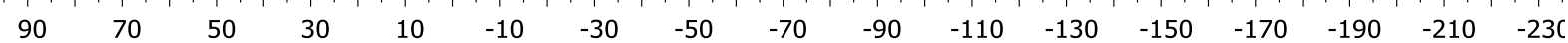


MALDI-TOF MS

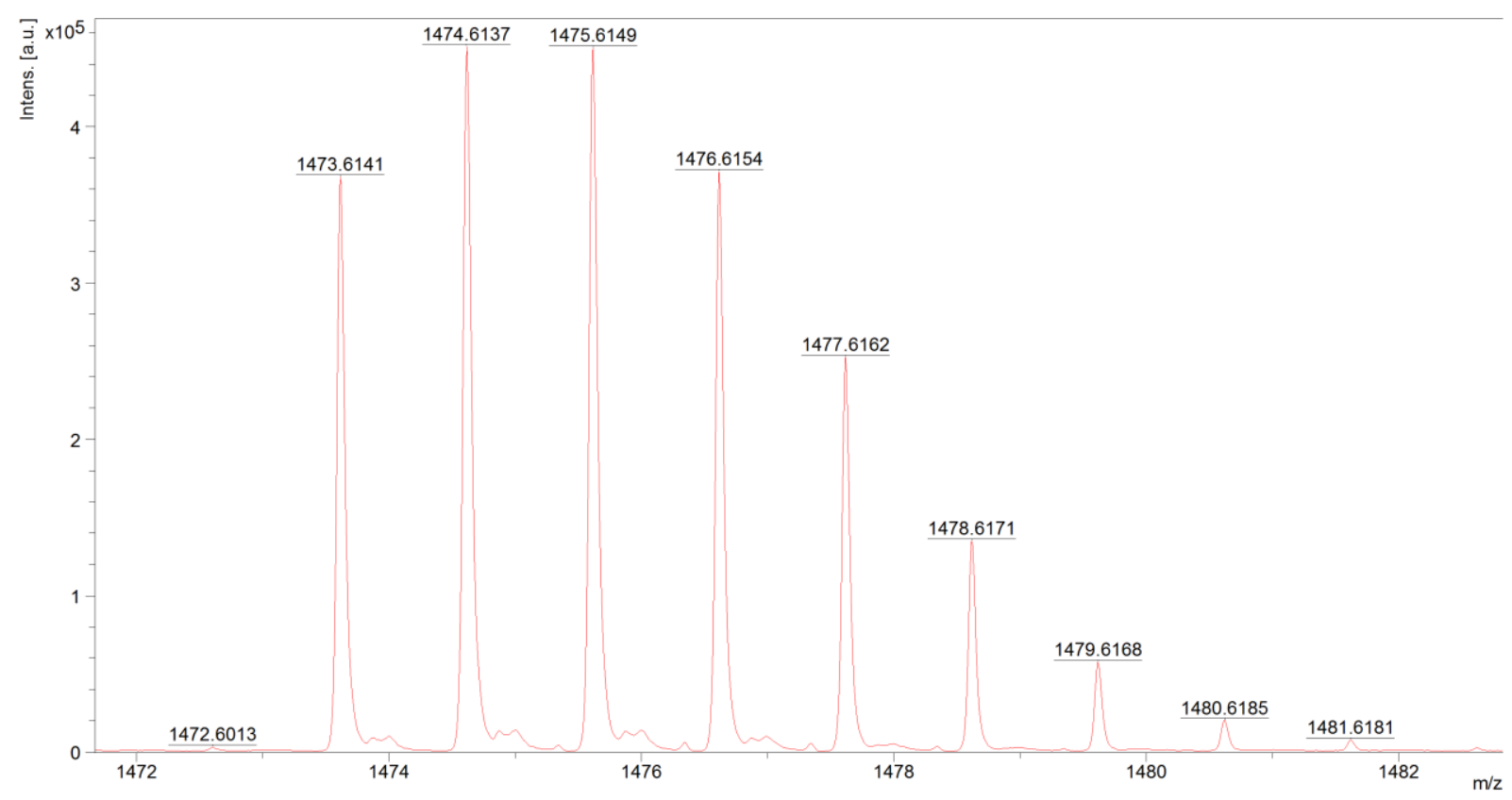


Compound 2a

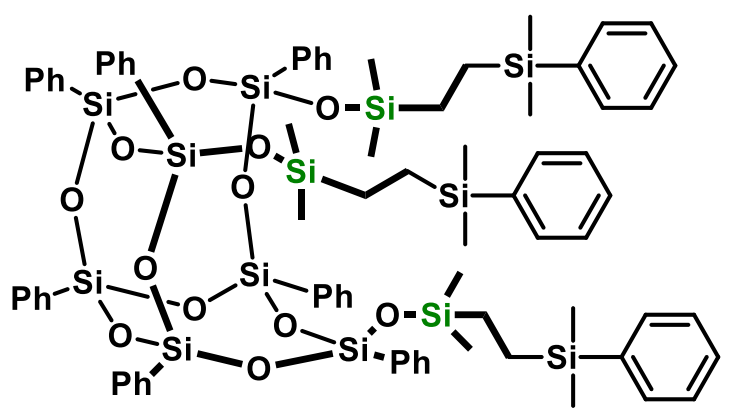

Chemical Formula: $\mathrm{C}_{78} \mathrm{H}_{98} \mathrm{O}_{12} \mathrm{Si}_{13}$

${ }^{1} \mathrm{H}$ NMR (300 MHz, Chloroform-d) $\delta 7.66-7.56(\mathrm{~m}, 4 \mathrm{H}), 7.53-7.28(\mathrm{~m}, 36 \mathrm{H}), 7.21-7.12(\mathrm{~m}, 10 \mathrm{H})$,

$0.75-0.65(\mathrm{~m}, 4 \mathrm{H}), 0.62-0.53(\mathrm{~m}, 6 \mathrm{H}), 0.46-0.38(\mathrm{~m}, 2 \mathrm{H}), 0.30(\mathrm{~s}, 14 \mathrm{H}), 0.27-0.07(\mathrm{~m}, 22 \mathrm{H})$.

${ }^{13} \mathrm{C} \mathrm{NMR}\left(75 \mathrm{MHz}, \mathrm{CDCl}_{3}\right) \delta 139.59,134.18,134.13,133.77,133.14,131.38,130.80,130.25,130.08$, $128.84,128.00,127.82,127.71,127.66,10.21,7.13,-0.11,-0.16,-3.45,-3.50$.

${ }^{29} \mathrm{Si} N \mathrm{NMR}\left(79 \mathrm{MHz}, \mathrm{CDCl}_{3}\right) \delta 12.43,-1.31,-77.33,-77.84,-78.12$.

MALDI-TOF MS (m/z): calcd. for $\mathrm{C}_{78} \mathrm{H}_{98} \mathrm{O}_{12} \mathrm{Si}_{13} \mathrm{Na} 1615,72$; found 1615,39.

${ }^{1} \mathrm{H}$ NMR (300 MHz, $\mathrm{CDCl}_{3}$ )

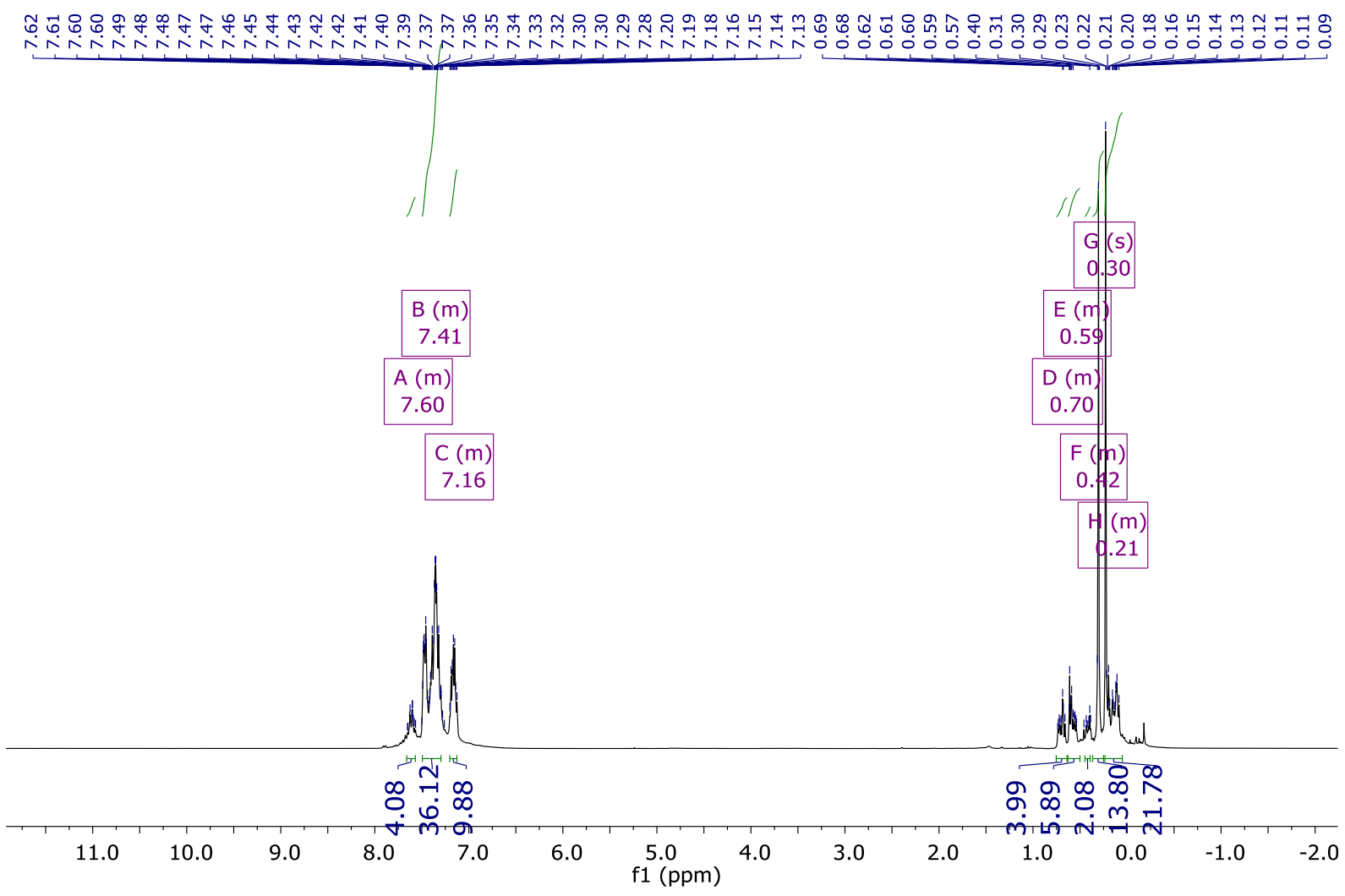


${ }^{13} \mathrm{C}$ NMR (101 MHz, $\mathrm{CDCl}_{3}$ )

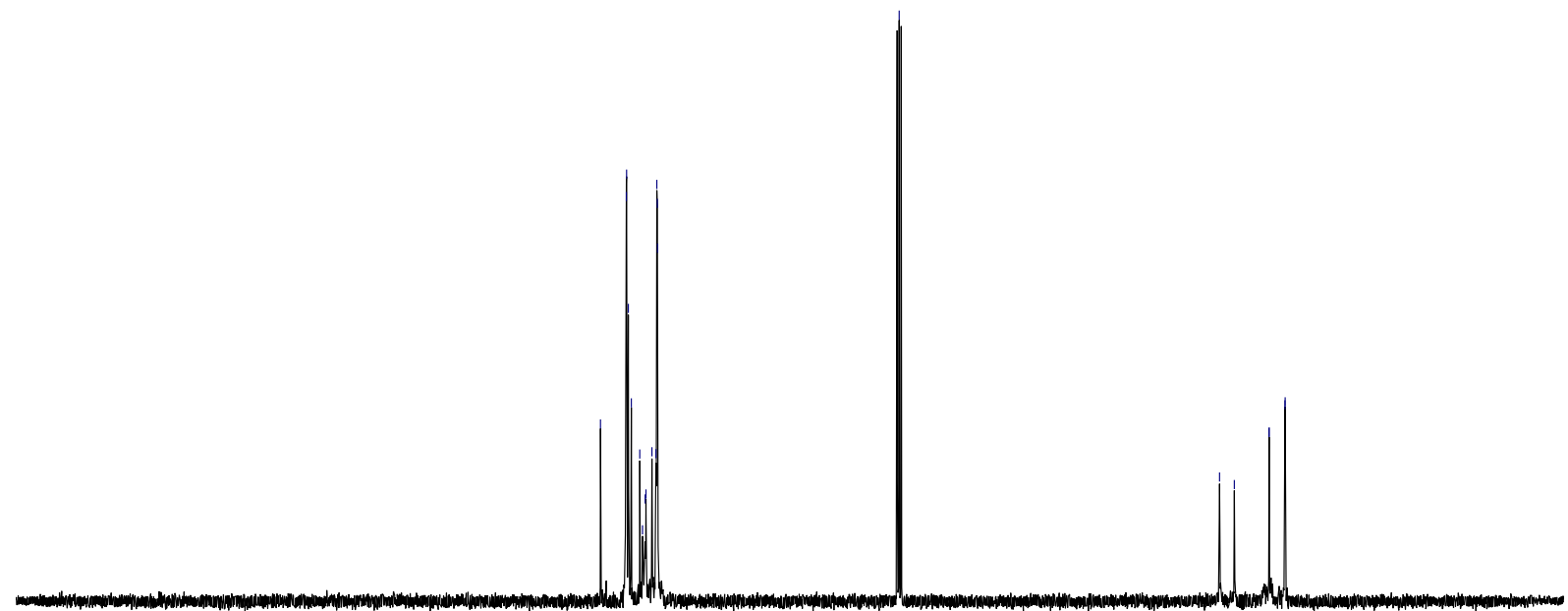

$\begin{array}{llllllllllllllllllllllllllllllllllllll}260 & 250 & 240 & 230 & 220 & 210 & 200 & 190 & 180 & 170 & 160 & 150 & 140 & 130 & 120 & 110 & 100 & 90 & 80 & 70 & 60 & 50 & 40 & 30 & 20 & 10 & 0 & -10 & -20 & -30 & -40 & -50 & -6\end{array}$

${ }^{29} \mathrm{Si}$ NMR (79 MHz, $\mathrm{CDCl}_{3}$ )
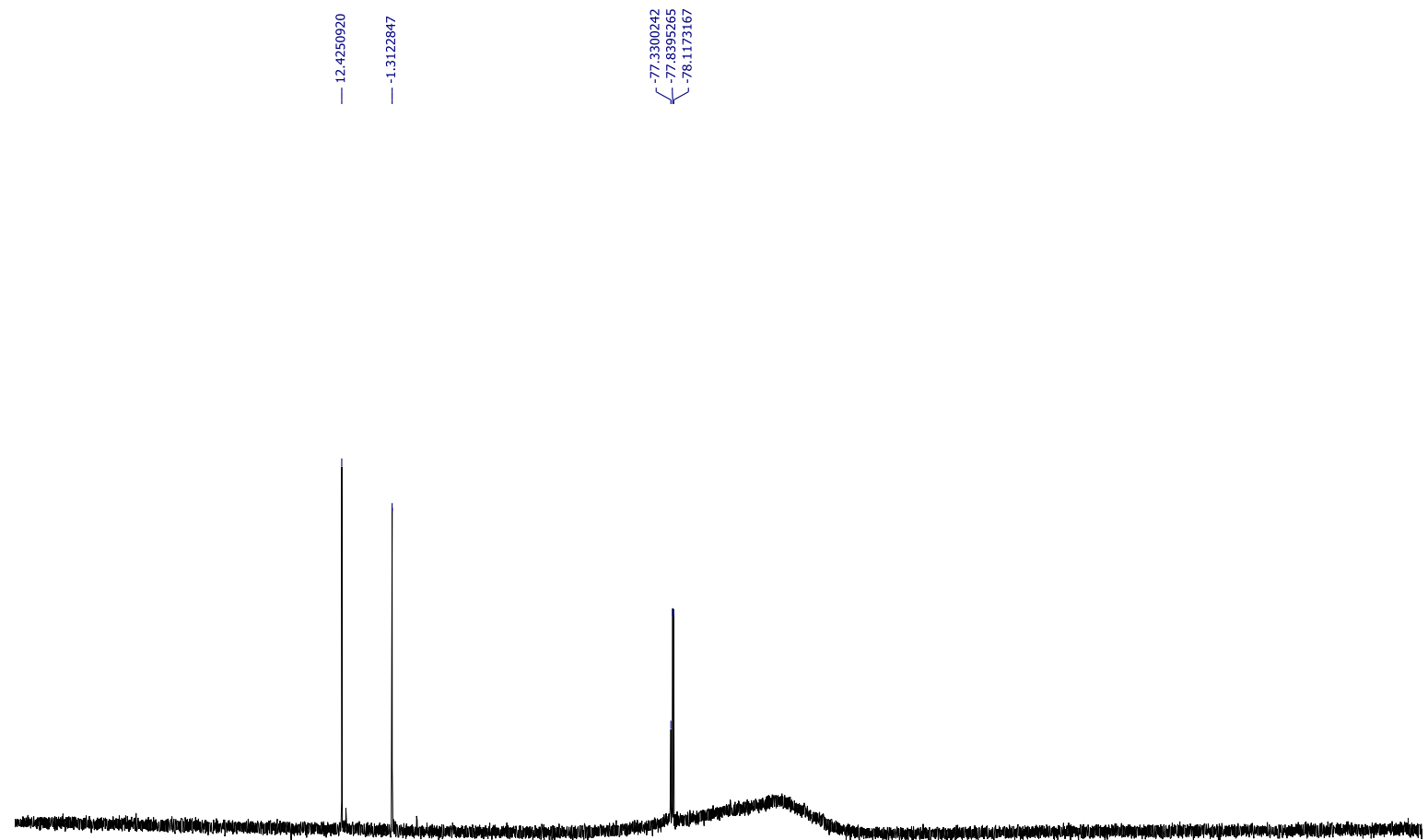

\begin{tabular}{llllllllllllllllllllllllllll}
\hline & 100 & 80 & 60 & 40 & 20 & 0 & -20 & -40 & -60 & -80 & -100 & -120 & -140 & -160 & -180 & -200 & -220 & -240 & -260 & -28 &
\end{tabular}


MALDI-TOF MS

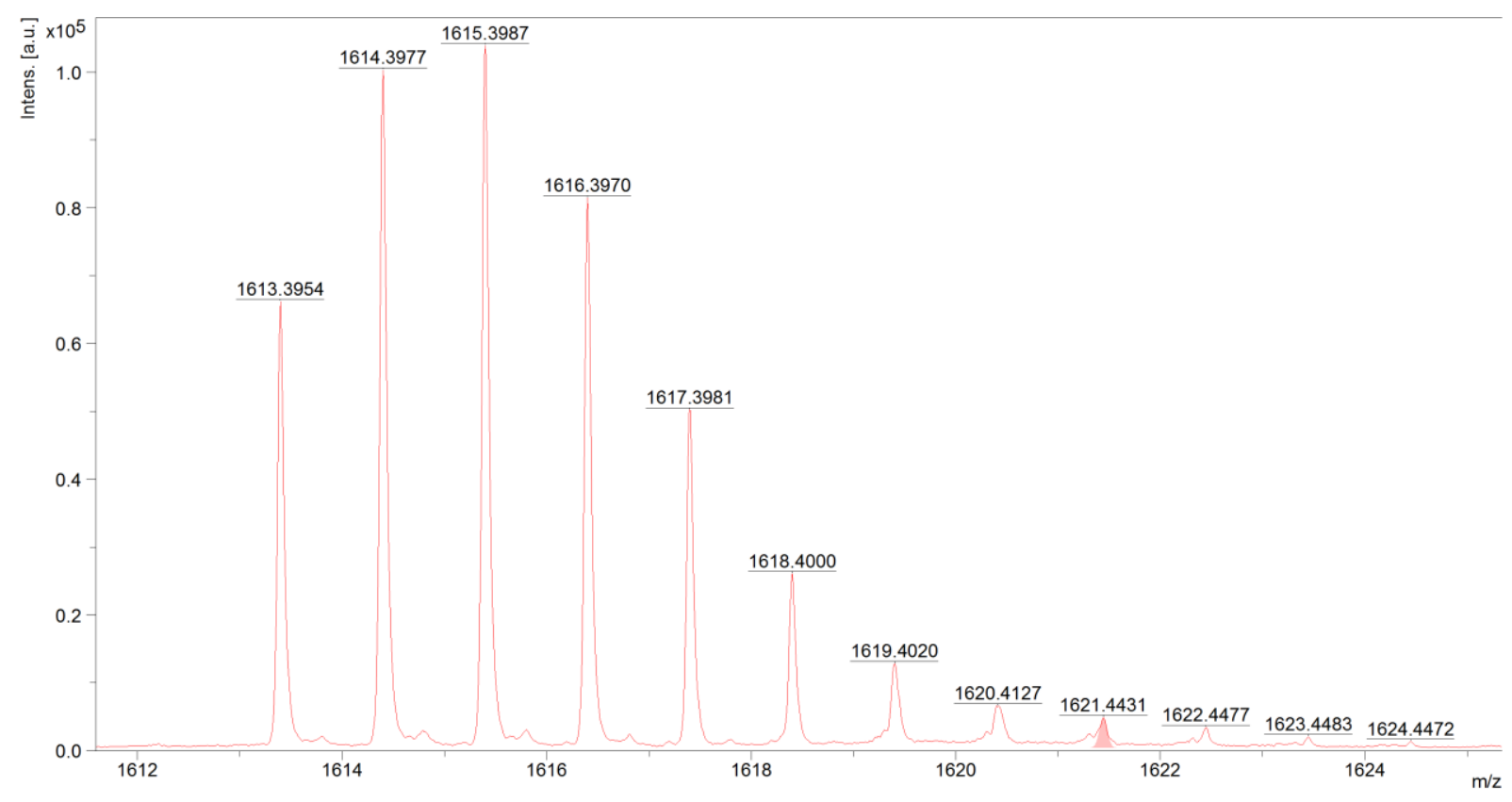


Compound $3 a$

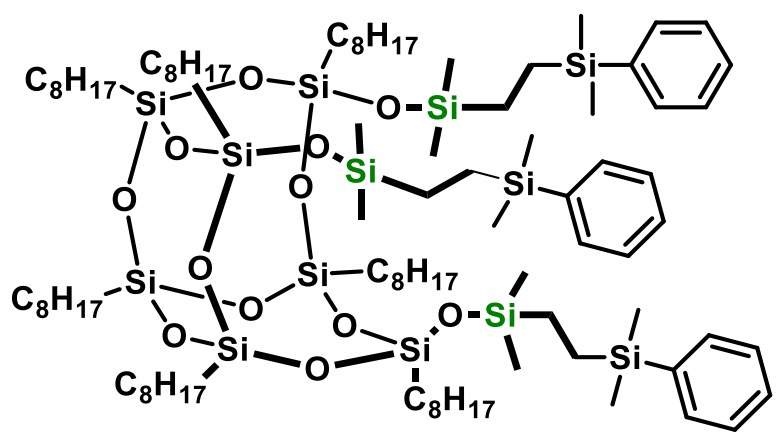

Chemical Formula: $\mathrm{C}_{92} \mathrm{H}_{182} \mathrm{O}_{12} \mathrm{Si}_{13}$

${ }^{1} \mathrm{H}$ NMR (300 MHz, Chloroform-d) $\delta 7.57-7.45(\mathrm{~m}, 6 \mathrm{H}), 7.38-7.30(\mathrm{~m}, 9 \mathrm{H}), 1.90-1.73(\mathrm{~m}, 7 \mathrm{H}), 1.35$ $-1.20(\mathrm{~m}, 7 \mathrm{H}), 1.18-1.07(\mathrm{~m}, 7 \mathrm{H}), 1.00(\mathrm{~d}, J=6.7 \mathrm{~Hz}, 18 \mathrm{H}), 0.90(\mathrm{~d}, J=2.8 \mathrm{~Hz}, 66 \mathrm{H}), 0.80-0.61(\mathrm{~m}$, $13 \mathrm{H}), 0.57-0.43(\mathrm{~m}, 13 \mathrm{H}), 0.24(\mathrm{~s}, 18 \mathrm{H}), 0.10(\mathrm{~s}, 18 \mathrm{H})$.

${ }^{13} \mathrm{C}$ NMR $\left(101 \mathrm{MHz}, \mathrm{CDCl}_{3}\right) \delta$ 133.77, 128.86, 127.82, 55.25, 54.62, 31.33, 30.45, 30.30, 29.61, 26.57, $25.87,25.49,25.36,25.27,25.06,10.24,7.25,-0.16,-3.40$.

${ }^{29} \mathrm{Si} \mathrm{NMR}\left(79 \mathrm{MHz}, \mathrm{CDCl}_{3}\right) \delta 9.51,-1.37,-67.53,-68.22$.

MALDI-TOF MS (m/z): calcd. for $\mathrm{C}_{92} \mathrm{H}_{182} \mathrm{O}_{12} \mathrm{Si}_{13} \mathrm{Na}$ 1868,55; found 1868,06.

${ }^{1} \mathrm{H}$ NMR (300 MHz, $\mathrm{CDCl}_{3}$ )

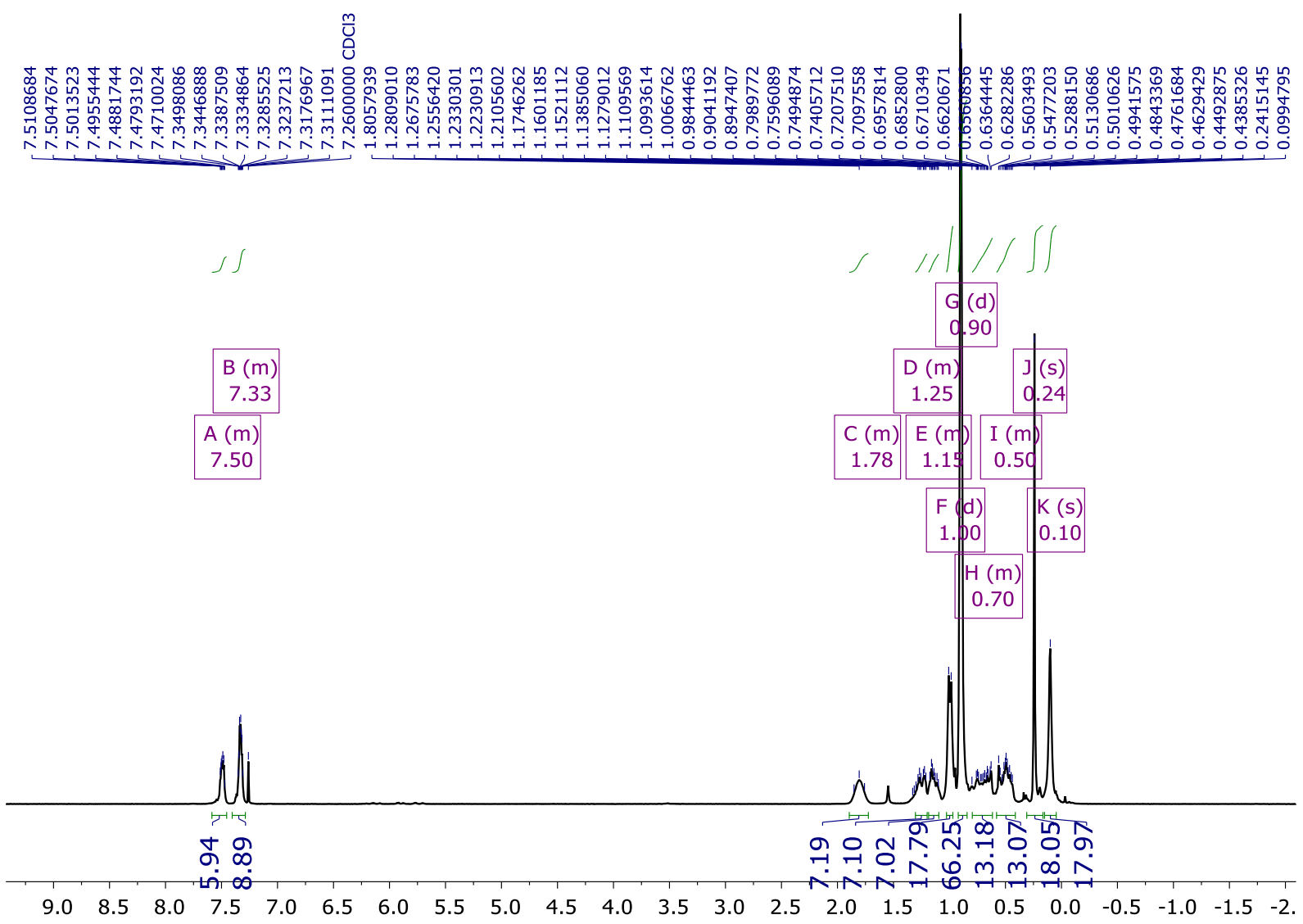


$\left.{ }^{13} \mathrm{C} \mathrm{NMR} \mathrm{(101} \mathrm{MHz,} \mathrm{CDCl}_{3}\right)$

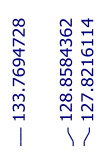

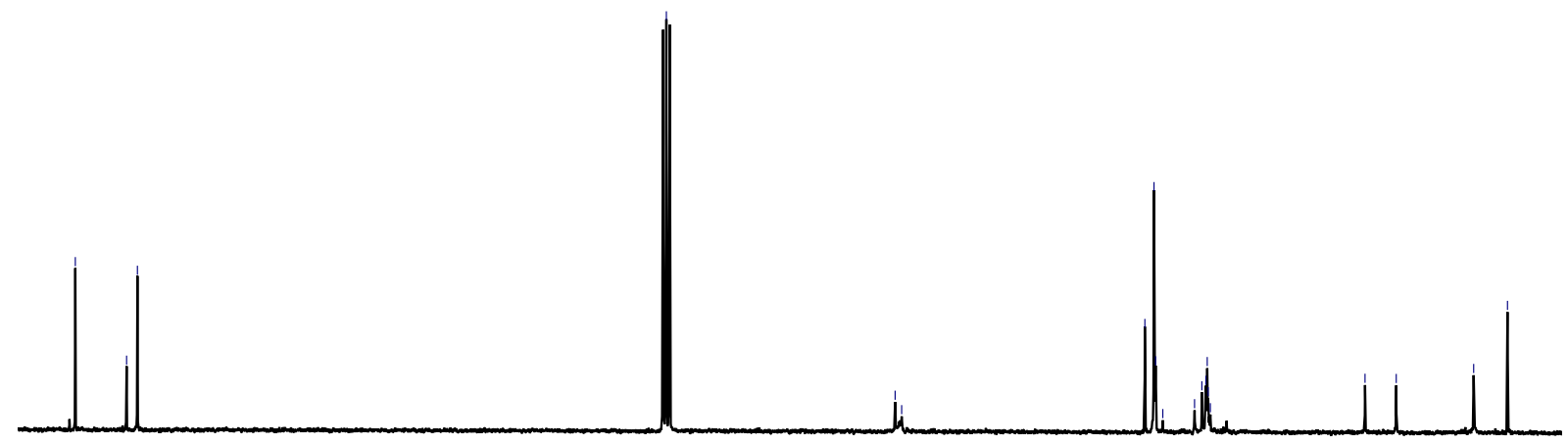

$\begin{array}{lllllllllllllllllllllllllllll}135 & 130 & 125 & 120 & 115 & 110 & 105 & 100 & 95 & 90 & 85 & 80 & 75 & 70 & 65 & 60 & 55 & 50 & 45 & 40 & 35 & 30 & 25 & 20 & 15 & 10 & 5 & 0 & -5\end{array}$ ${ }^{29} \mathrm{Si}$ NMR $\left(79 \mathrm{MHz}, \mathrm{CDCl}_{3}\right)$<smiles>O=S(=O)(I)OI</smiles>

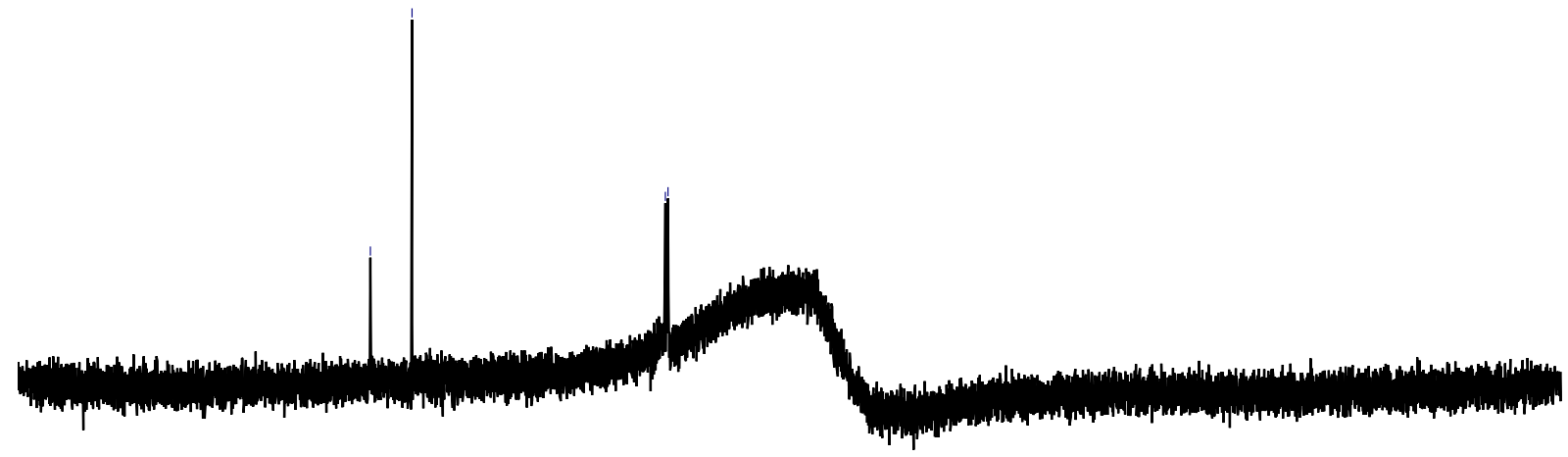

$\begin{array}{llllllllllllllllllllllll}00 & 80 & 60 & 40 & 20 & 0 & -20 & -40 & -60 & -80 & -100 & -120 & -140 & -160 & -180 & -200 & -220 & -240 & -260 & -280 & -31\end{array}$ 
MALDI-TOF MS

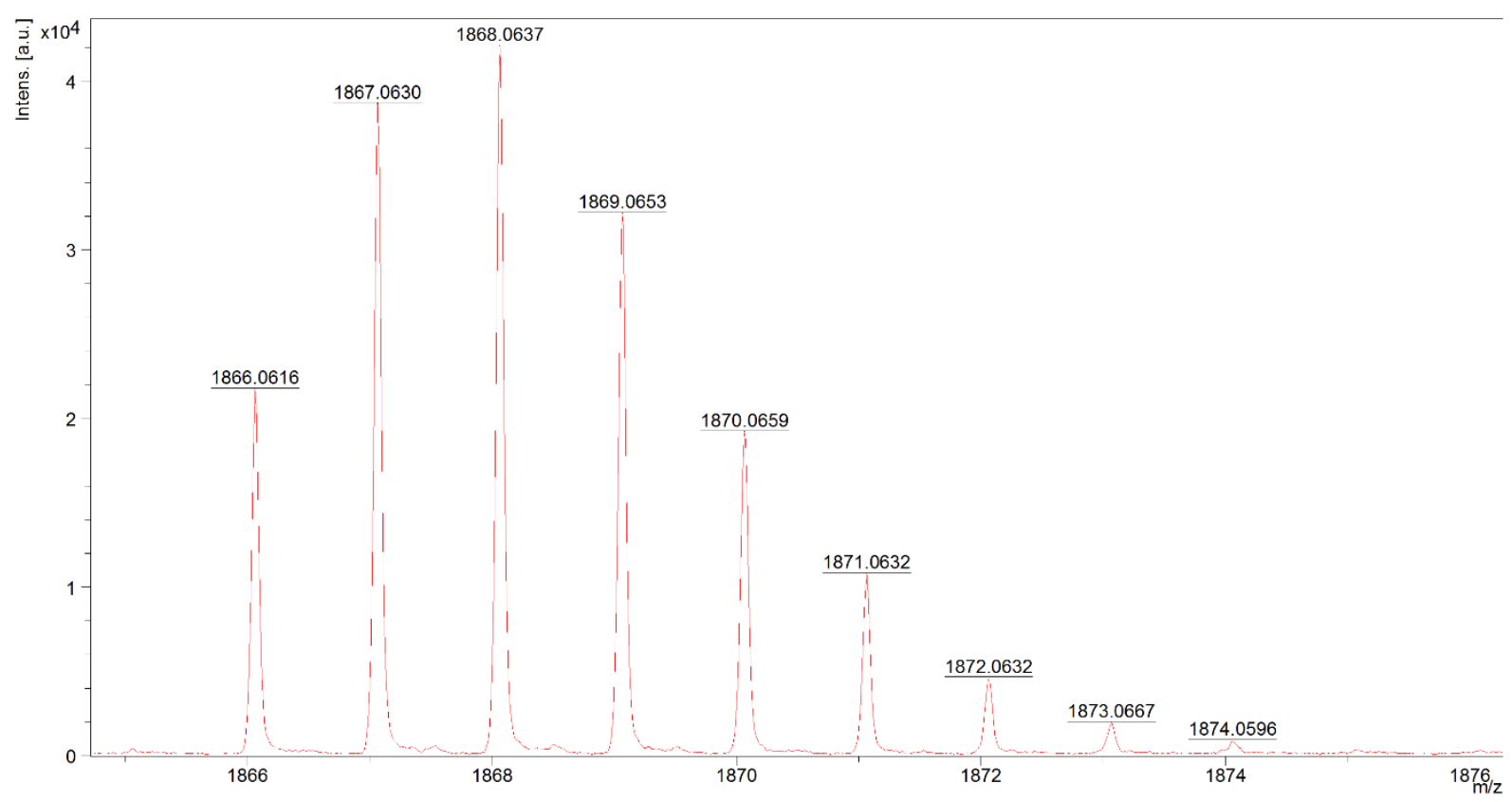


Compound $4 a$

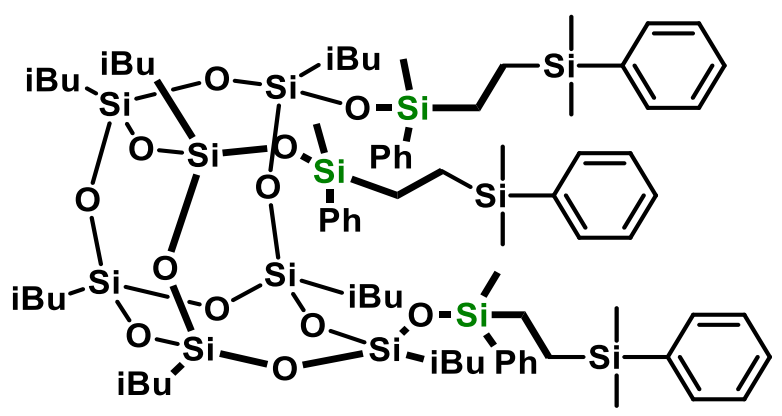

Chemical Formula: $\mathrm{C}_{79} \mathrm{H}_{132} \mathrm{O}_{12} \mathrm{Si}_{13}$

${ }^{1} \mathrm{H}$ NMR (300 MHz, Chloroform-d) $\delta 7.49-7.32(\mathrm{~m}, 12 \mathrm{H}), 7.31-7.12(\mathrm{~m}, 18 \mathrm{H}), 1.88-1.64(\mathrm{~m}, 7 \mathrm{H})$, $0.96-0.78(\mathrm{~m}, 42 \mathrm{H}), 0.67-0.41(\mathrm{~m}, 26 \mathrm{H}), 0.24(\mathrm{~s}, 32 \mathrm{H}), 0.21-0.11(\mathrm{~m}, 21 \mathrm{H}), 0.07(\mathrm{~s}, 3 \mathrm{H})$.

${ }^{13} \mathrm{C}$ NMR $\left(75 \mathrm{MHz}, \mathrm{CDCl}_{3}\right) \delta 139.51,139.48,138.41,133.78,133.64,129.17,128.88,127.81,127.64$, $29.87,26.21,26.05,25.79,25.21,24.18,23.98,22.61,9.04,7.03,-1.57,-3.43$.

${ }^{29} \mathrm{Si}$ NMR $\left(79 \mathrm{MHz}, \mathrm{CDCl}_{3}\right) \delta-0.41,-0.48,-0.52,-1.29,-1.32,-67.22,-67.26,-67.37,-67.41,-67.54,-$ $67.60,-67.62$.

MALDI-TOF MS (m/z): calcd. for $\mathrm{C}_{79} \mathrm{H}_{132} \mathrm{O}_{12} \mathrm{Si}_{13} \mathrm{Na}$ 1662,01; found 1661,66.

\section{${ }^{1} \mathrm{H}$ NMR $\left(300 \mathrm{MHz}, \mathrm{CDCl}_{3}\right)$}

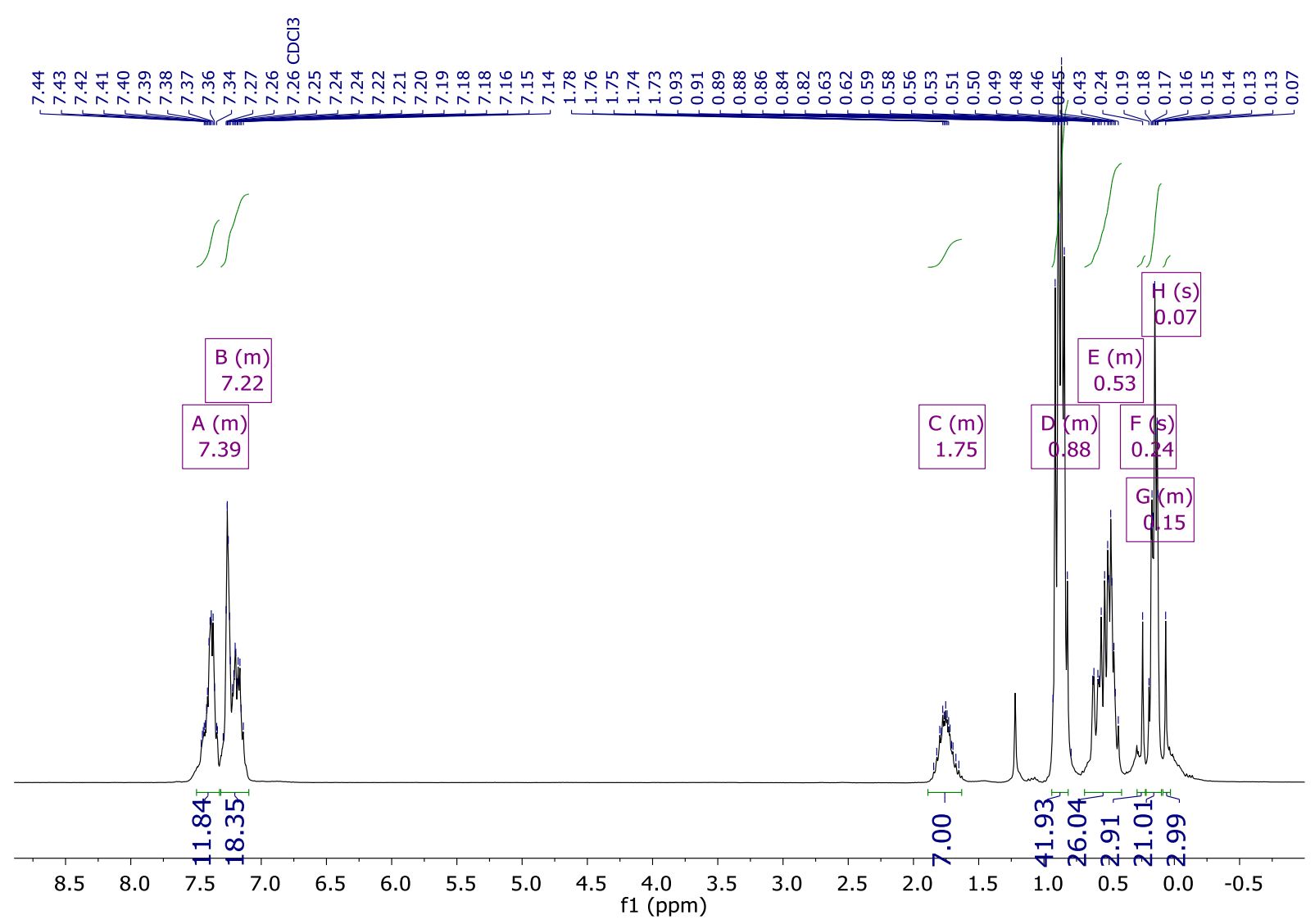


${ }^{13} \mathrm{C}$ NMR (101 MHz, $\mathrm{CDCl}_{3}$ )

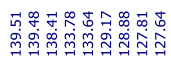

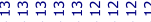

जiv

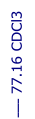

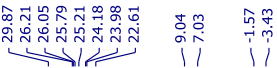

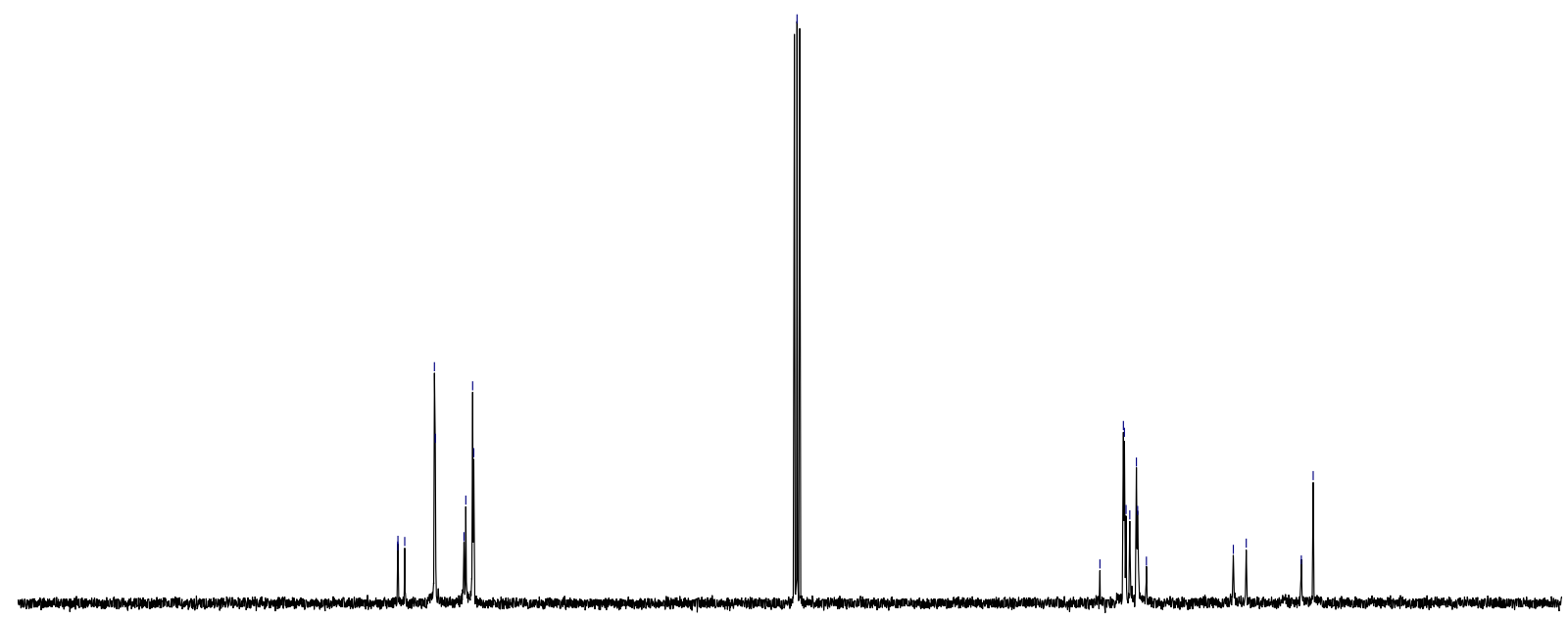

$\begin{array}{llllllllllllllllllllllllllll}190 & 180 & 170 & 160 & 150 & 140 & 130 & 120 & 110 & 100 & 90 & 80 & 70 & 60 & 50 & 40 & 30 & 20 & 10 & 0 & -10 & -20 & -30 & -40\end{array}$

${ }^{29} \mathrm{Si} \mathrm{NMR} \mathrm{(79} \mathrm{MHz,} \mathrm{CDCl}_{3}$ )
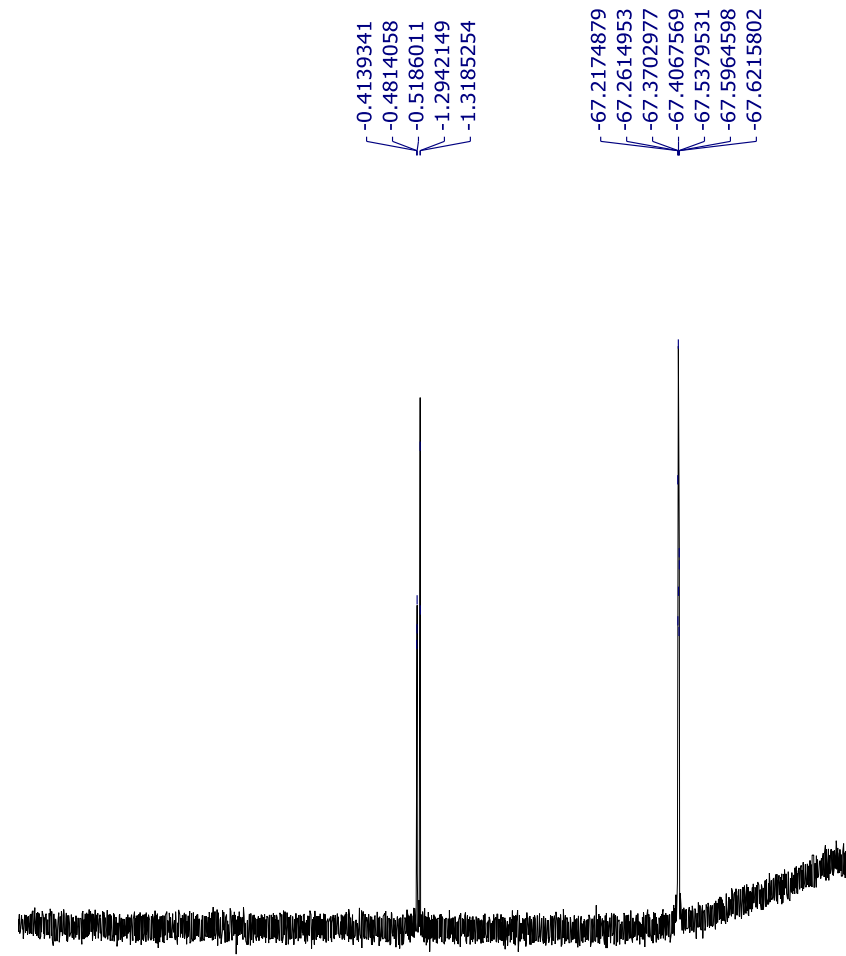

$\begin{array}{llllllllllllllllllllll}00 & 80 & 60 & 40 & 20 & 0 & -20 & -40 & -60 & -80 & -100 & -120 & -140 & -160 & -180 & -200 & -220 & -240 & -260 & -280 & -3\end{array}$ 
MALDI-TOF MS

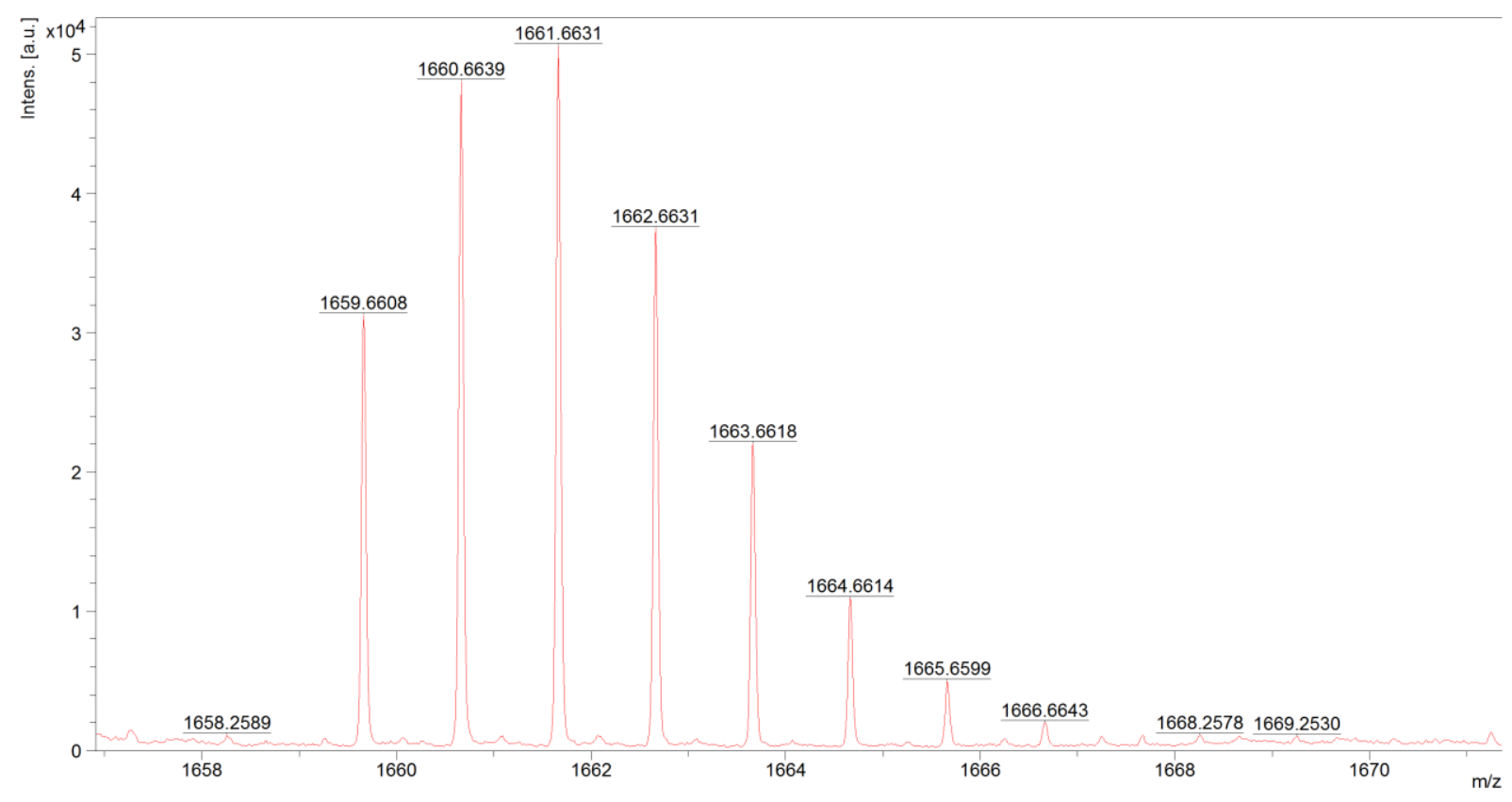


Compound 5a

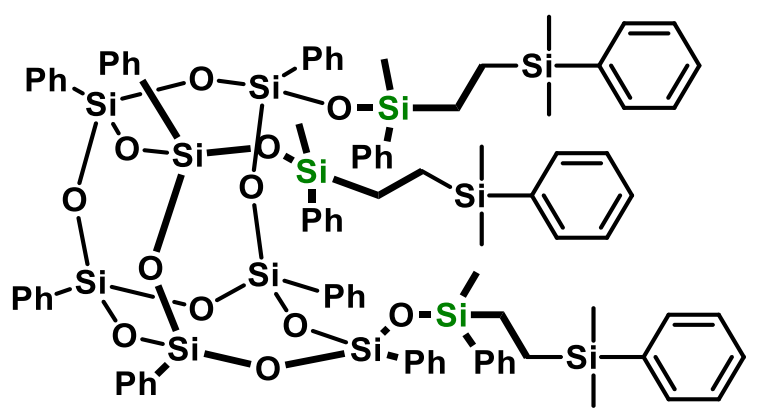

Chemical Formula: $\mathrm{C}_{93} \mathrm{H}_{104} \mathrm{O}_{12} \mathrm{Si}_{13}$

${ }^{1} \mathrm{H}$ NMR (300 MHz, Chloroform-d) $\delta 7.68-7.27(\mathrm{~m}, 30 \mathrm{H}), 7.25-7.00(\mathrm{~m}, 20 \mathrm{H}), 1.01-0.76(\mathrm{~m}, 1 \mathrm{H})$, $0.68(\mathrm{~s}, 2 \mathrm{H}), 0.62-0.51(\mathrm{~m}, 5 \mathrm{H}), 0.50-0.42(\mathrm{~m}, 4 \mathrm{H}), 0.41-0.32(\mathrm{~m}, 4 \mathrm{H}), 0.30-0.23(\mathrm{~m}, 3 \mathrm{H}), 0.22-$ $0.16(\mathrm{~m}, 2 \mathrm{H}), 0.16--0.10(\mathrm{~m}, 18 \mathrm{H})$.

${ }^{13} \mathrm{C} \mathrm{NMR}\left(101 \mathrm{MHz}, \mathrm{CDCl}_{3}\right) \delta 134.32,134.30,134.22,134.14,133.79,133.76,133.74,133.71,133.63$, $133.54,129.24,128.77,127.95,127.84,127.80,127.75,127.72,127.67,127.63,8.96,7.93,6.79,-3.62$. ${ }^{29} \mathrm{Si} N \mathrm{NMR}\left(79 \mathrm{MHz}, \mathrm{CDCl}_{3}\right) \delta 1.81,-1.42,-75.47,-77.70,-77.91$.

MALDI-TOF MS (m/z): calcd. for $\mathrm{C}_{93} \mathrm{H}_{104} \mathrm{O}_{12} \mathrm{Si}_{13} \mathrm{Na} 1801,94$; found 1801,44.

\section{${ }^{1} \mathrm{H} \mathrm{NMR}\left(300 \mathrm{MHz}, \mathrm{CDCl}_{3}\right)$}

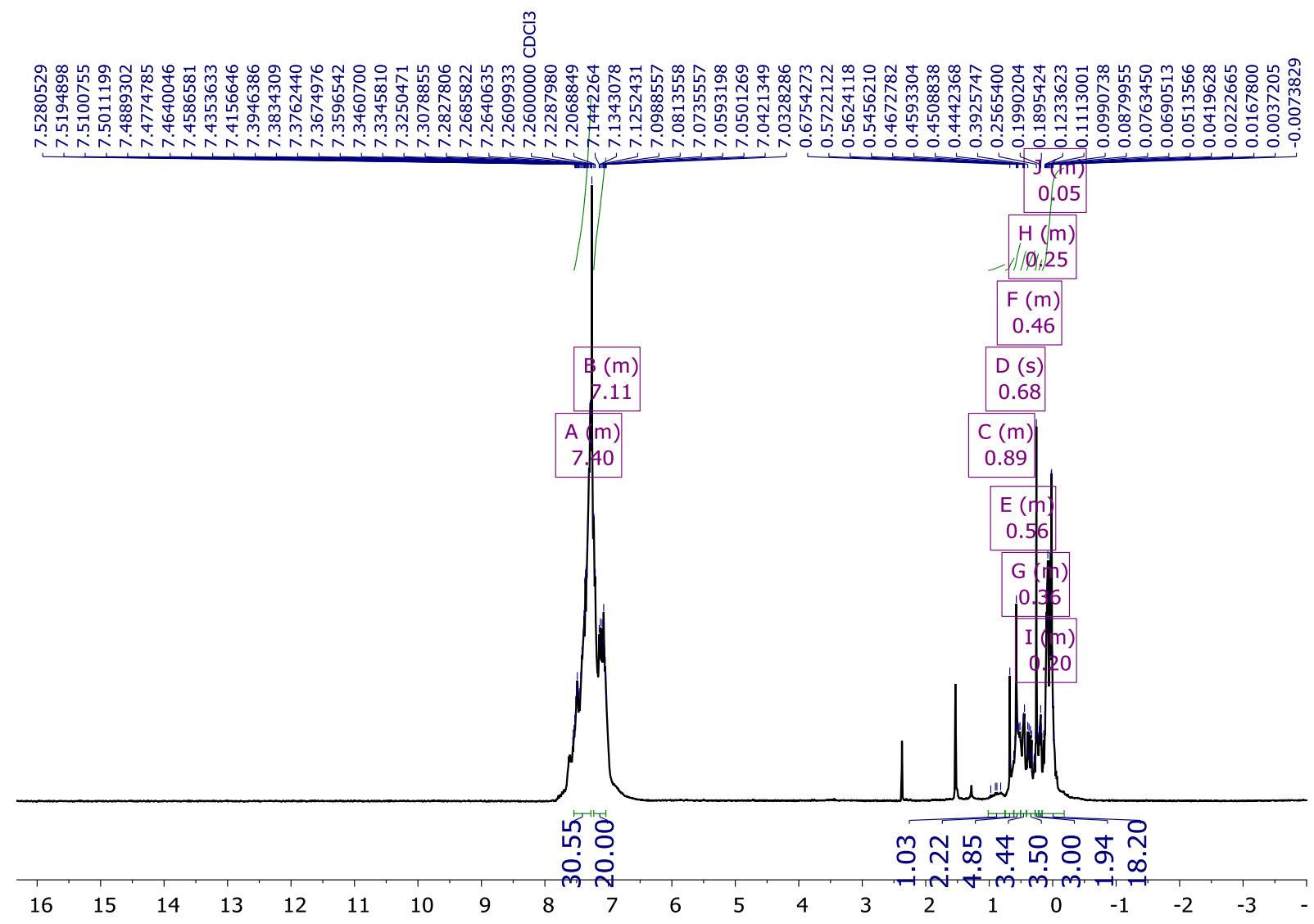




$$
-
$$


MALDI-TOF MS

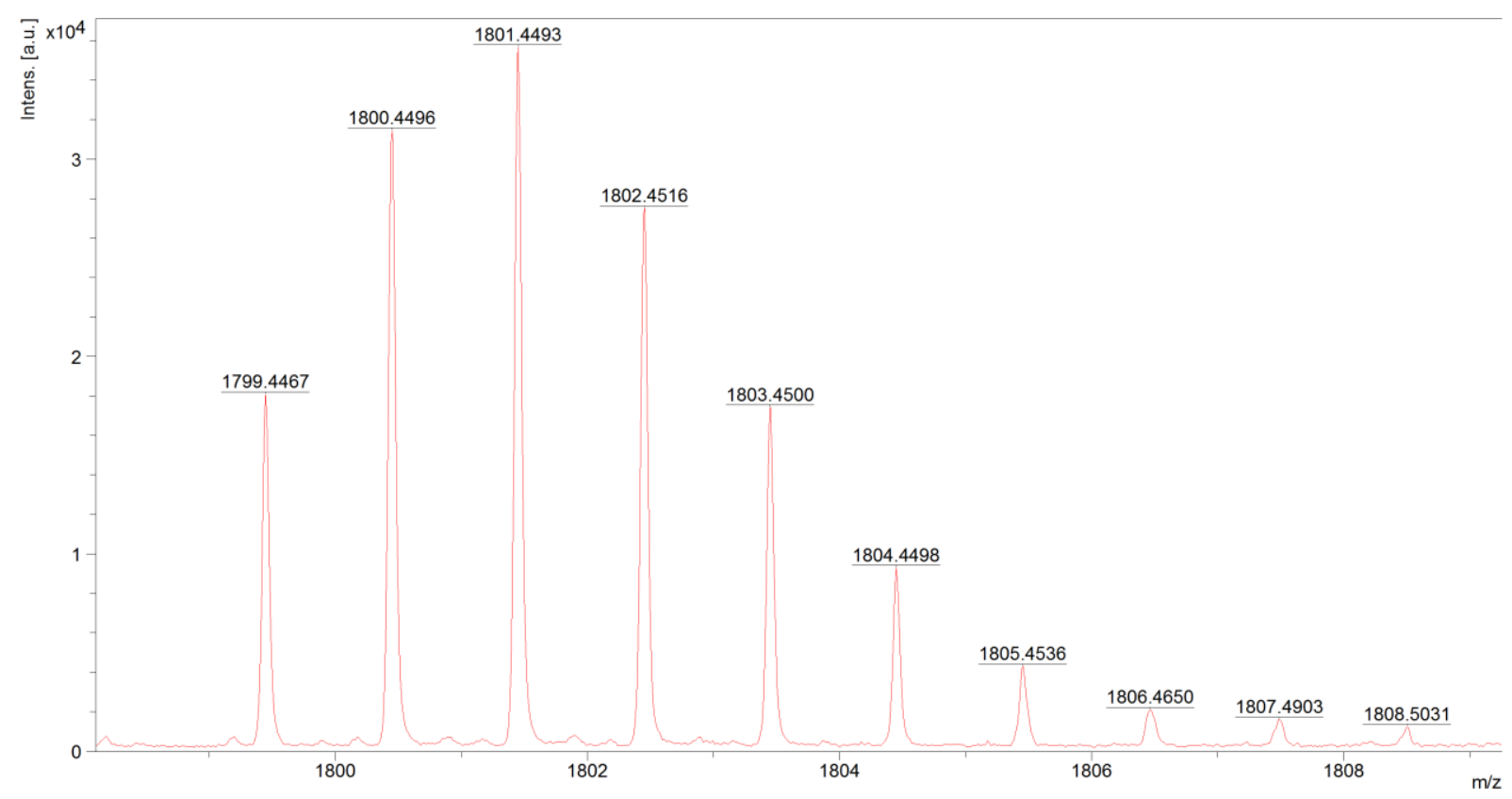


Compound 6a<smiles>C[Si](C)(CC[Si](C)(C)c1ccccc1)c1ccccc1</smiles>

Chemical Formula: $\mathrm{C}_{107} \mathrm{H}_{188} \mathrm{O}_{12} \mathrm{Si}_{13}$

${ }^{1} \mathrm{H}$ NMR (300 MHz, Chloroform-d) $\delta 7.62-7.27(\mathrm{~m}, 24 \mathrm{H}), 7.25-7.11(\mathrm{~m}, 6 \mathrm{H}), 1.92-1.67(\mathrm{~m}, 7 \mathrm{H}), 1.36$ $-1.07(\mathrm{~m}, 14 \mathrm{H}), 1.05-0.70(\mathrm{~m}, 98 \mathrm{H}), 0.66-0.46(\mathrm{~m}, 12 \mathrm{H}), 0.39--0.04(\mathrm{~m}, 27 \mathrm{H})$.

${ }^{13} \mathrm{C}$ NMR $\left(101 \mathrm{MHz}, \mathrm{CDCl}_{3}\right) \delta 139.47,134.33,133.77,133.69,129.14,128.92,128.86,127.95,127.84$, $127.80,127.65,55.31,55.25,55.02,54.67,31.33,31.28,30.42$, 30.30, 25.85, 25.46, 25.30, 25.04, 9.10, $8.99,7.94,7.06,7.02,-1.55,-2.25,-3.41$.

${ }^{29} \mathrm{Si}$ NMR $\left(79 \mathrm{MHz}, \mathrm{CDCl}_{3}\right) \delta$-0.59, -1.23, -1.31, -67.49, -67.79, -67.91, -68.08, -68.23, -68.23.

MALDI-TOF MS (m/z): calcd. for $\mathrm{C}_{107} \mathrm{H}_{188} \mathrm{O}_{12} \mathrm{Si}_{13} \mathrm{Na} 2054,76$; found 2054,10.

\section{${ }^{1} \mathrm{H}$ NMR $\left(300 \mathrm{MHz}, \mathrm{CDCl}_{3}\right)$}
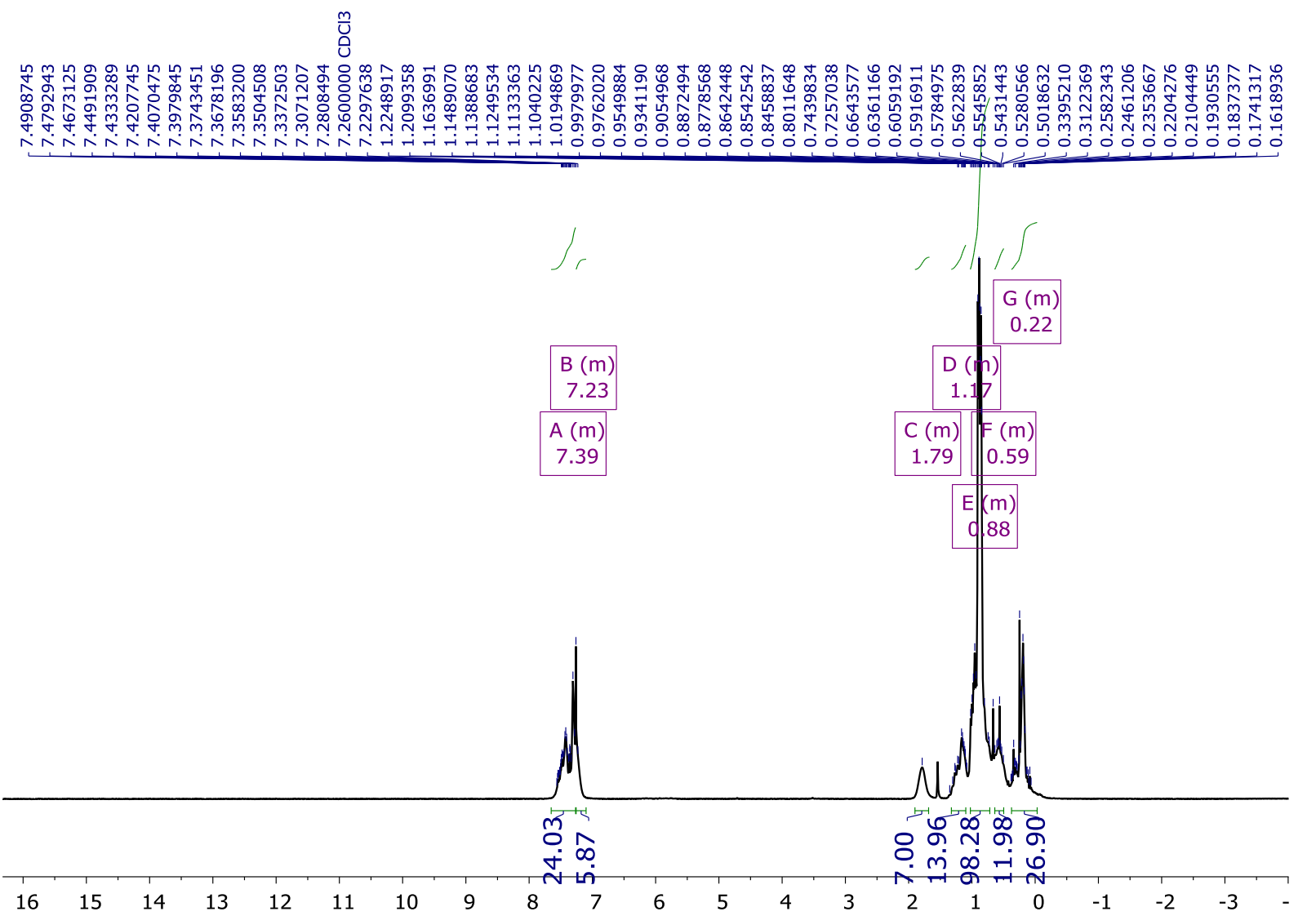
${ }^{13} \mathrm{C}$ NMR (101 MHz, $\left.\mathrm{CDCl}_{3}\right)$
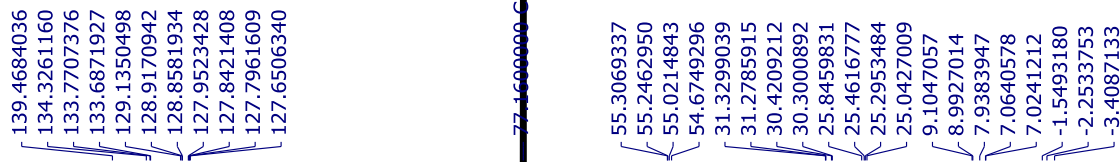

$\begin{array}{lllllllllllllllllllllll}210 & 200 & 190 & 180 & 170 & 160 & 150 & 140 & 130 & 120 & 110 & 100 & 90 & 80 & 70 & 60 & 50 & 40 & 30 & 20 & 10 & 0 & -10\end{array}$ ${ }^{29}$ Si NMR (79 MHz, $\left.\mathrm{CDCl}_{3}\right)$
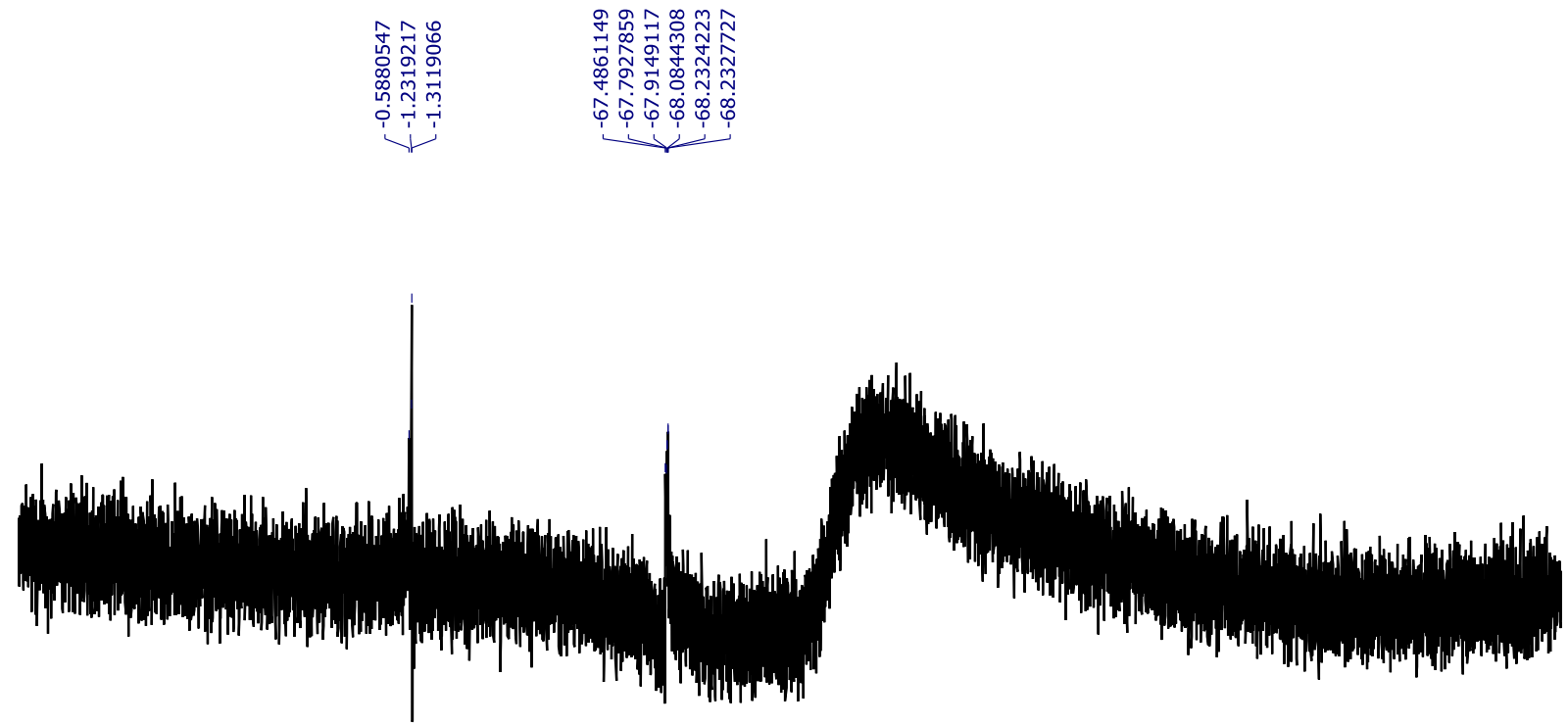

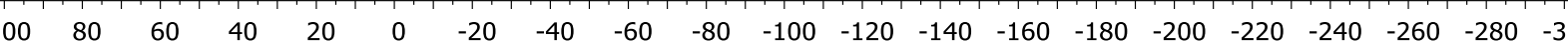


MALDI-TOF MS

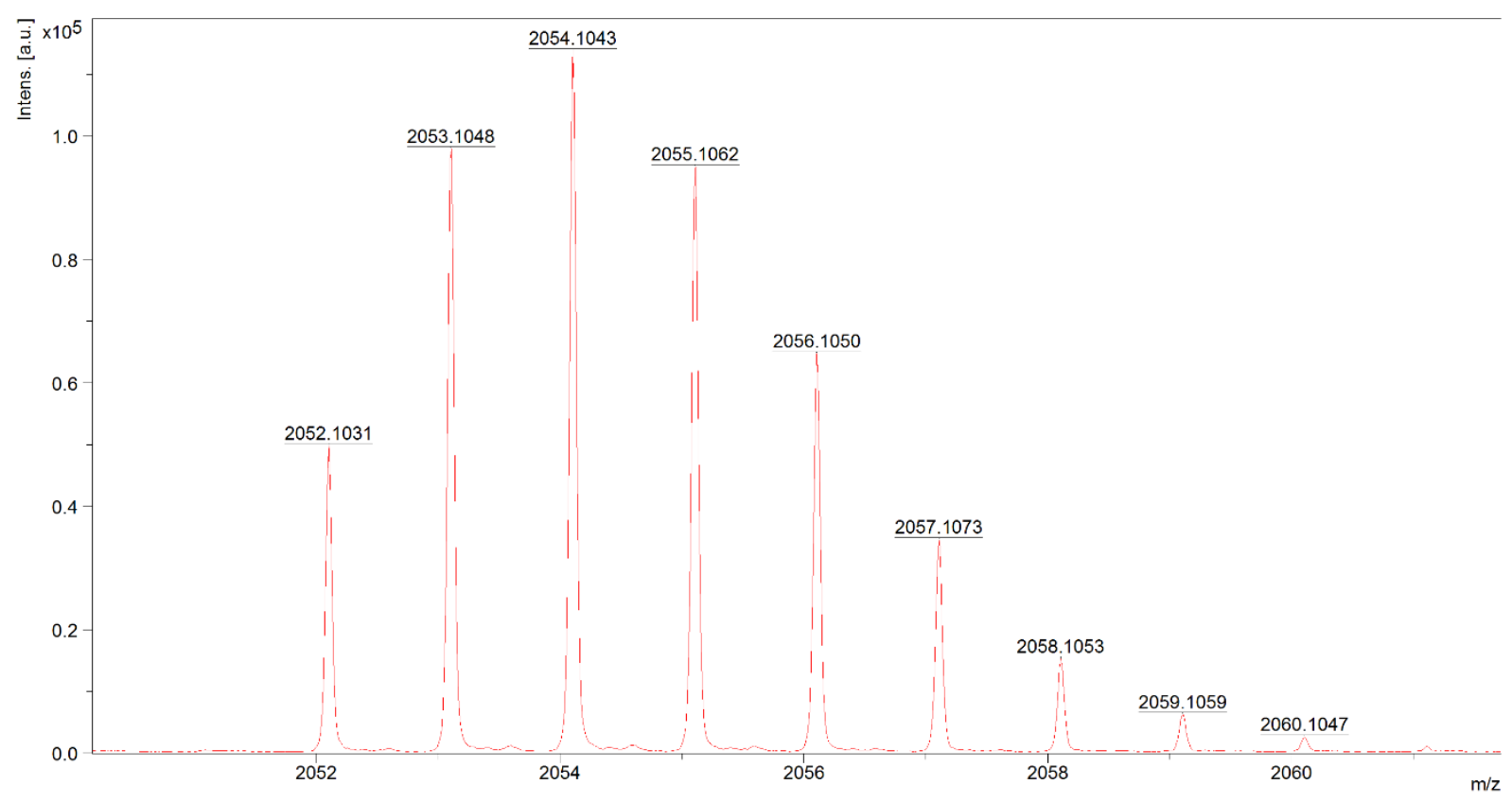


Compound 7a

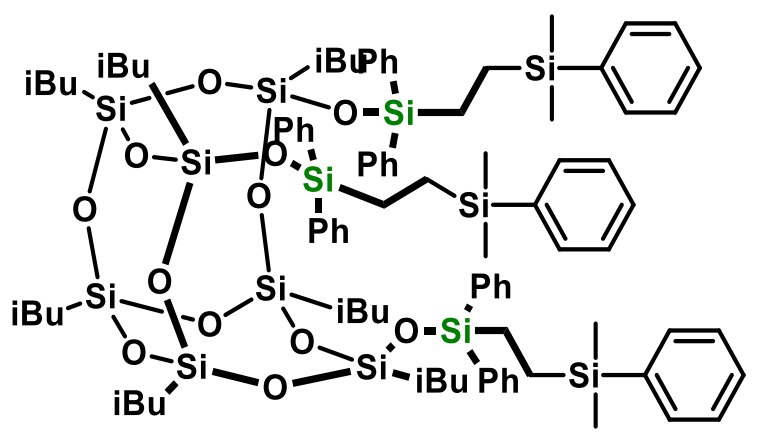

Chemical Formula: $\mathrm{C}_{94} \mathrm{H}_{138} \mathrm{O}_{12} \mathrm{Si}_{13}$

${ }^{1} \mathrm{H}$ NMR (300 MHz, Chloroform-d) $\delta 7.63-7.50(\mathrm{~m}, 13 \mathrm{H}), 7.49-7.41(\mathrm{~m}, 8 \mathrm{H}), 7.39-7.32(\mathrm{~m}, 8 \mathrm{H}), 7.21$

$-7.12(\mathrm{~m}, 15 \mathrm{H}), 1.84(\mathrm{~m}, 7 \mathrm{H}), 1.09-0.82(\mathrm{~m}, 48 \mathrm{H}), 0.77-0.55(\mathrm{~m}, 18 \mathrm{H}), 0.46-0.30(\mathrm{~m}, 2 \mathrm{H}), 0.20(\mathrm{~s}$, $18 \mathrm{H})$.

${ }^{13} \mathrm{C}$ NMR $\left(75 \mathrm{MHz}, \mathrm{CDCl}_{3}\right) \delta 139.38,136.07,134.51,133.80,129.42,128.85,127.78,127.75,29.87$, $26.20,26.07,25.83,25.48,24.09,24.01,22.68,7.53,6.99,-3.46,-3.49$.

${ }^{29} \mathrm{Si} \mathrm{NMR}\left(79 \mathrm{MHz}, \mathrm{CDCl}_{3}\right) \delta-1.24,-11.61,-66.21,-67.21,-67.52$.

MALDI-TOF MS (m/z): calcd. for $\mathrm{C}_{94} \mathrm{H}_{138} \mathrm{O}_{12} \mathrm{Si}_{13} \mathrm{Na}$ 1848,22; found 1847,50.

\section{${ }^{1} \mathrm{H}$ NMR $\left(300 \mathrm{MHz}, \mathrm{CDCl}_{3}\right)$}

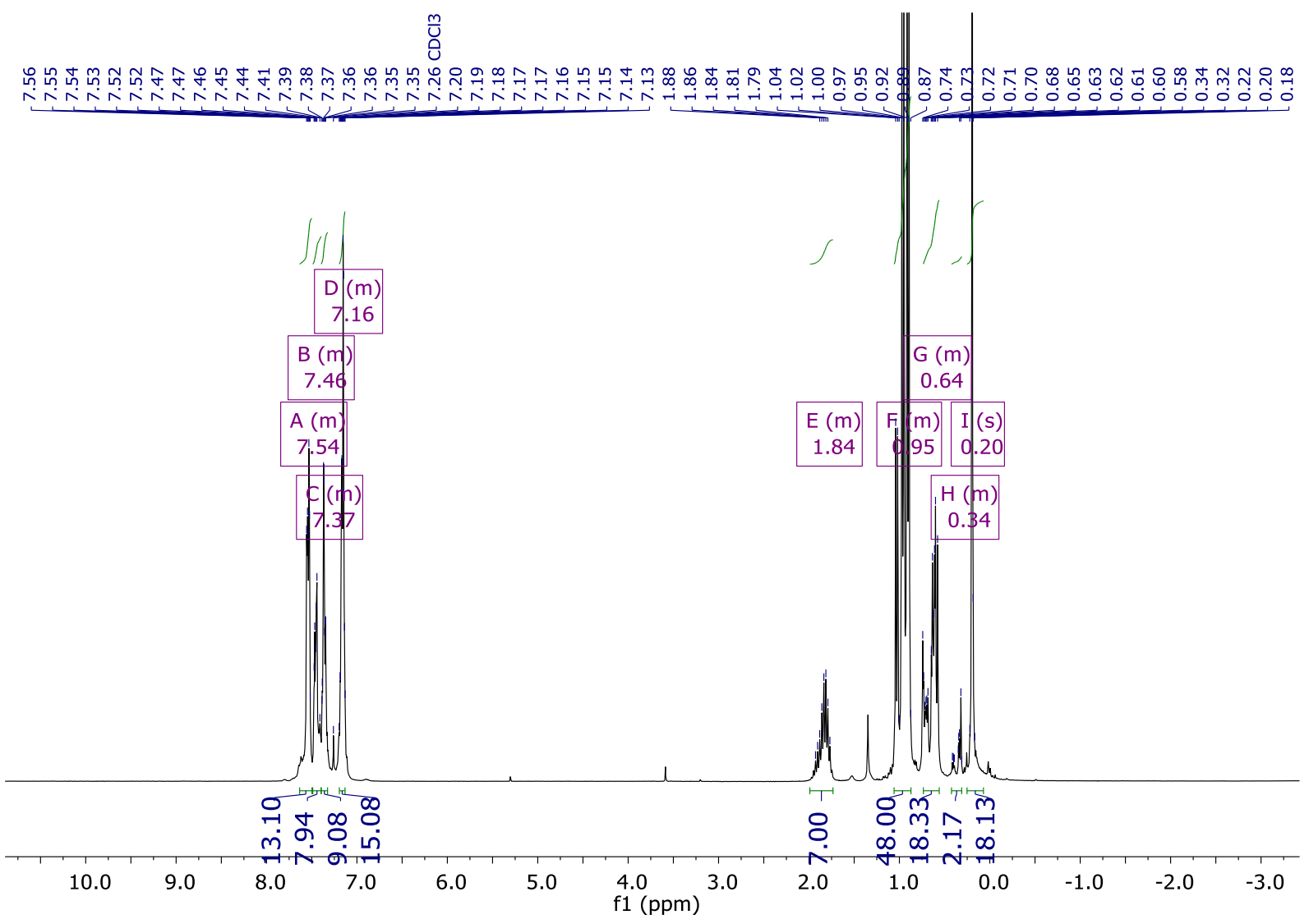


${ }^{13} \mathrm{C}$ NMR (101 MHz, $\mathrm{CDCl}_{3}$ )

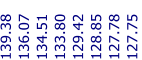

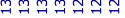

皮位

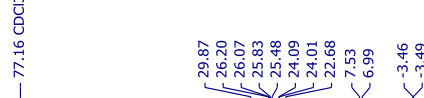

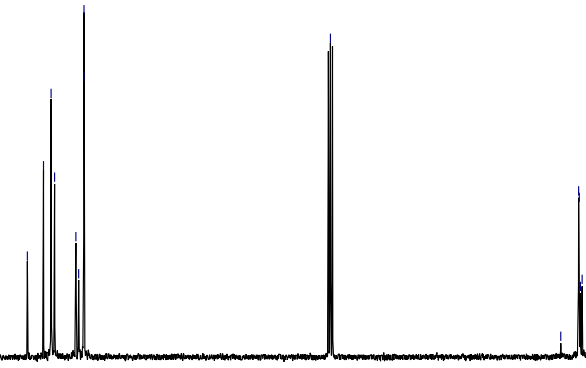

$\begin{array}{llllllllllllllllllllllllllllllllllllllll}260 & 250 & 240 & 230 & 220 & 210 & 200 & 190 & 180 & 170 & 160 & 150 & 140 & 130 & 120 & 110 & 100 & 90 & 80 & 70 & 60 & 50 & 40 & 30 & 20 & 10 & 0 & -10 & -20 & -30 & -40 & -50 & -6\end{array}$

${ }^{29} \mathrm{Si}$ NMR (79 MHz, $\mathrm{CDCl}_{3}$ )

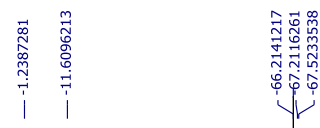

$\begin{array}{rlllllllllllllllllllllllllll}70 & 50 & 30 & 10 & -10 & -30 & -50 & -70 & -90 & -110 & -130 & -150 & -170 & -190 & -210 & -230 & -250 & -270 & -25\end{array}$ 
MALDI-TOF MS

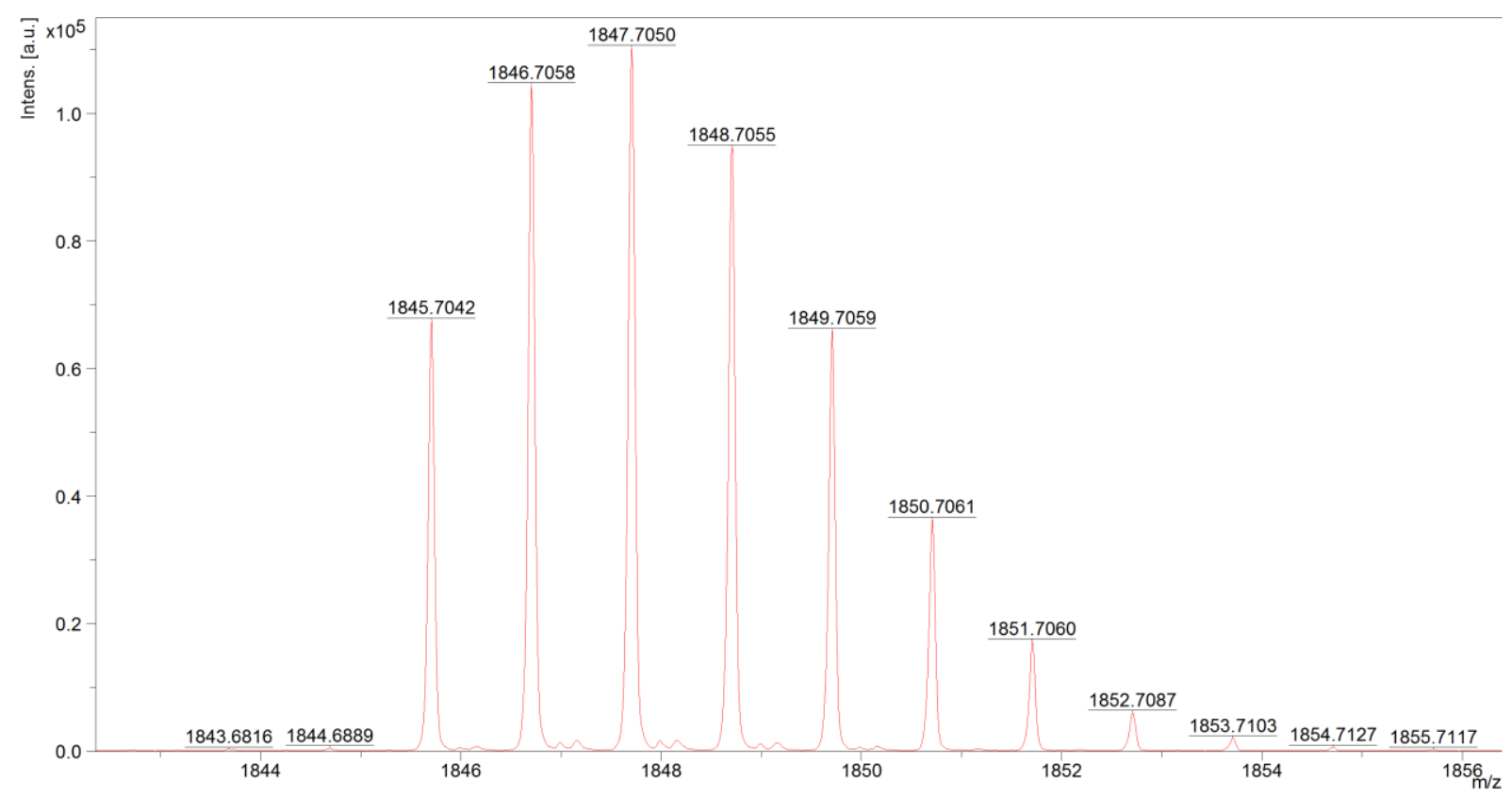


Compound 8a

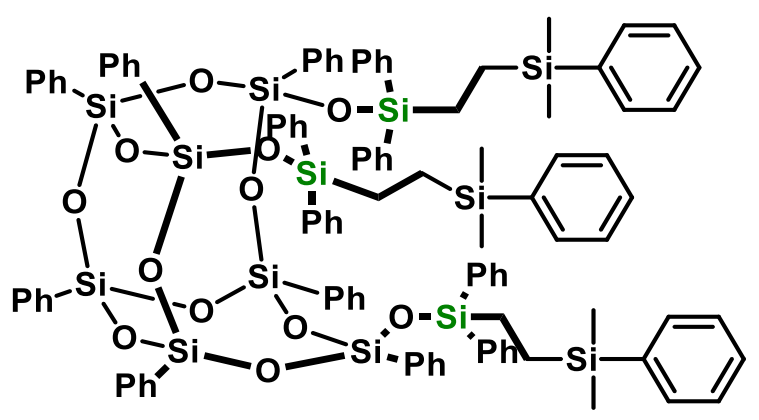

Chemical Formula: $\mathrm{C}_{108} \mathrm{H}_{110} \mathrm{O}_{12} \mathrm{Si}_{13}$

${ }^{1} \mathrm{H}$ NMR (300 MHz, Chloroform-d) $\delta 7.67-7.27(\mathrm{~m}, 33 \mathrm{H}), 7.25-6.84(\mathrm{~m}, 47 \mathrm{H}), 1.02-0.71(\mathrm{~m}, 4 \mathrm{H})$, $0.67(\mathrm{~s}, 2 \mathrm{H}), 0.62-0.49(\mathrm{~m}, 4 \mathrm{H}), 0.46-0.33(\mathrm{~m}, 3 \mathrm{H}), 0.31-0.21(\mathrm{~m}, 2 \mathrm{H}), 0.21--0.34(\mathrm{~m}, 15 \mathrm{H})$.

${ }^{13} \mathrm{C} \mathrm{NMR}\left(101 \mathrm{MHz}, \mathrm{CDCl}_{3}\right) \delta 135.85,134.50,134.33,134.32,134.25,134.18,133.79,133.74,129.50$, $129.19,128.74,128.38,127.95,127.84,127.75,127.70,127.44,7.49,6.65,-3.64$.

${ }^{29} \mathrm{Si} \mathrm{NMR}\left(79 \mathrm{MHz}, \mathrm{CDCl}_{3}\right) \delta-1.37,-9.49,-76.77,-76.81,-76.92$.

MALDI-TOF MS (m/z): calcd. for $\mathrm{C}_{108} \mathrm{H}_{110} \mathrm{O}_{12} \mathrm{Si}_{13} \mathrm{Na} 1988,15$; found 1987,49.

${ }^{1} \mathrm{H}$ NMR (300 MHz, $\left.\mathrm{CDCl}_{3}\right)$

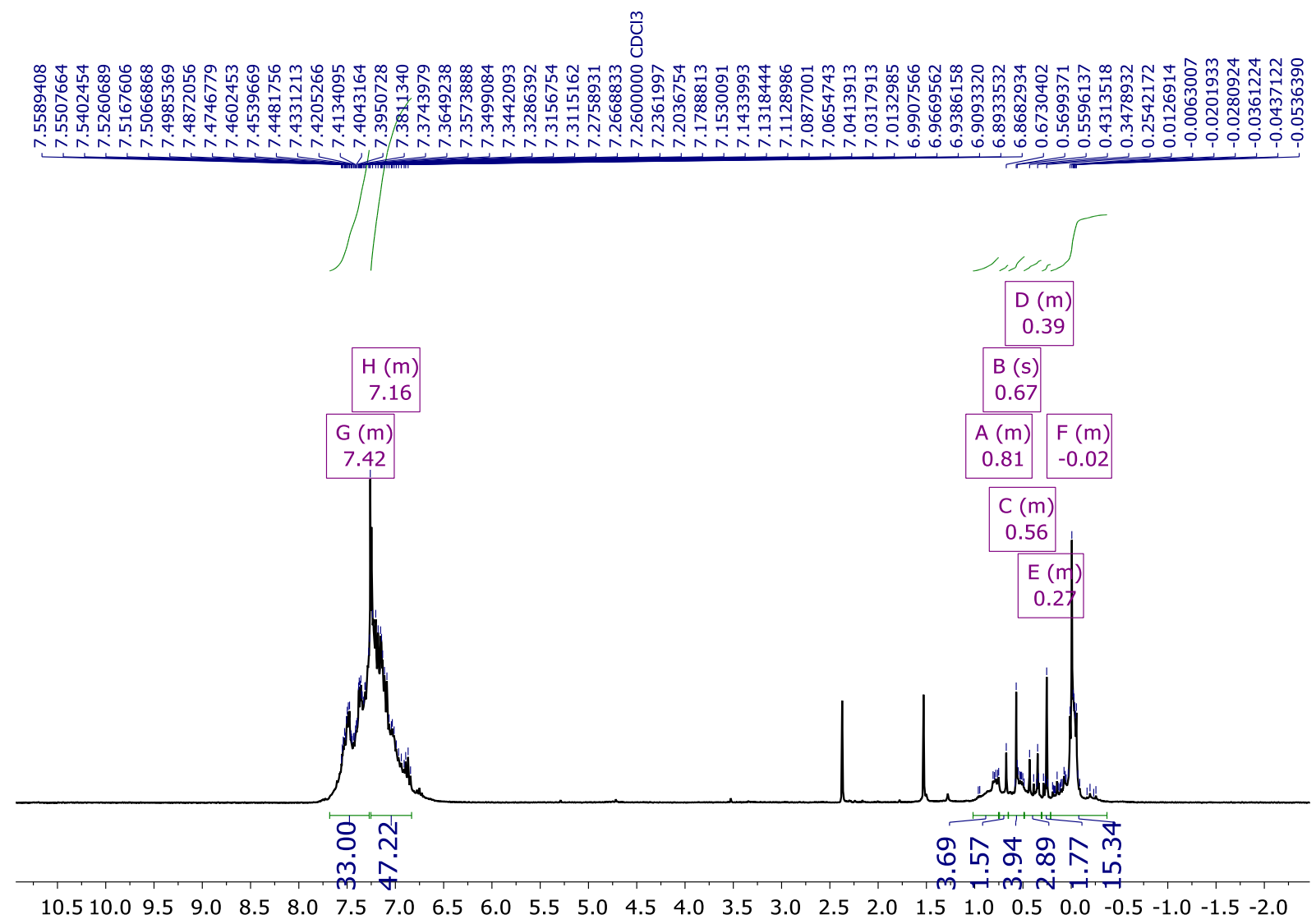


${ }^{13} \mathrm{C}$ NMR (101 MHz, $\mathrm{CDCl}_{3}$ )
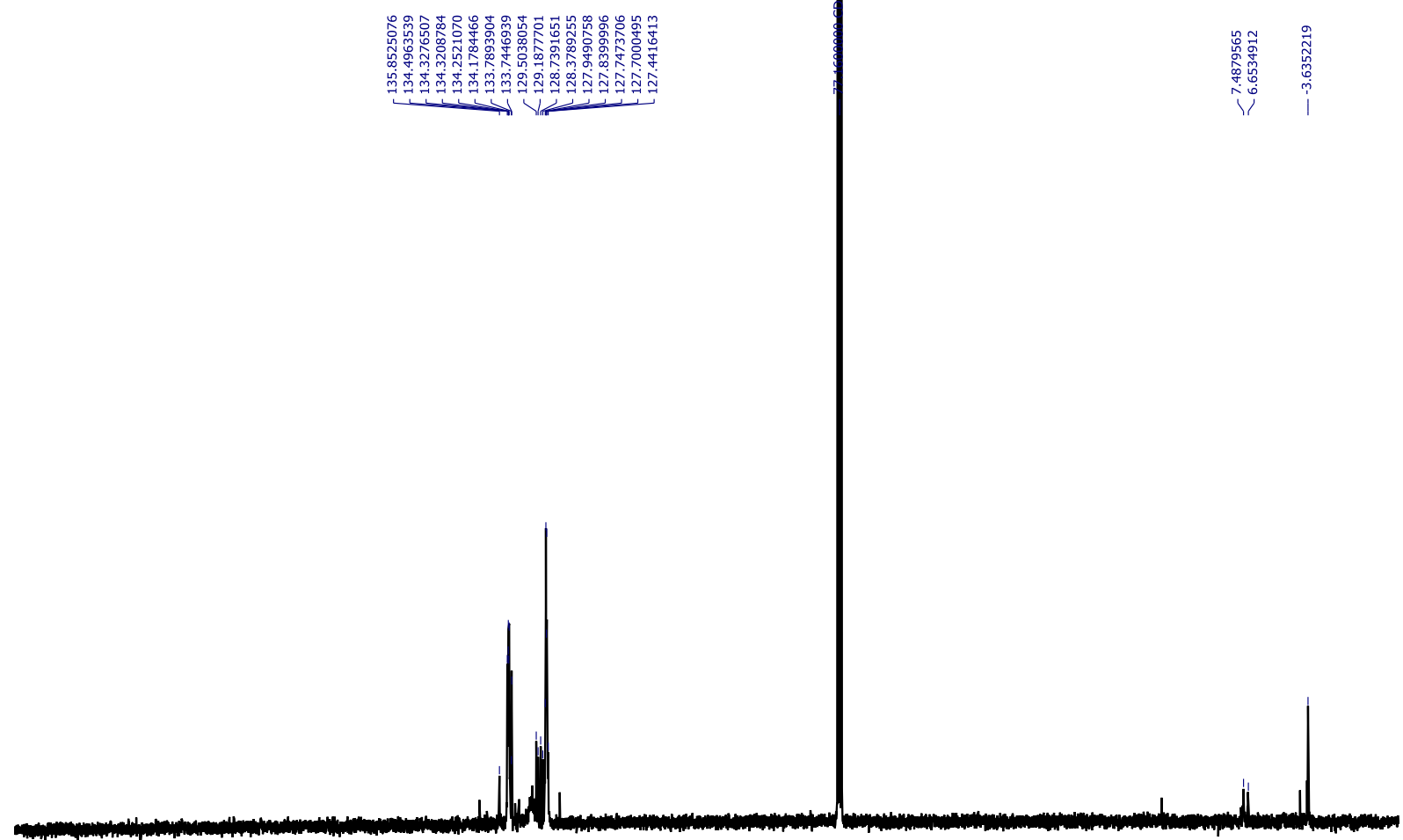

$\begin{array}{lllllllllllllllllllllll}210 & 200 & 190 & 180 & 170 & 160 & 150 & 140 & 130 & 120 & 110 & 100 & 90 & 80 & 70 & 60 & 50 & 40 & 30 & 20 & 10 & 0 & -10\end{array}$

${ }^{29} \mathrm{Si} \mathrm{NMR} \mathrm{(79} \mathrm{MHz,} \mathrm{CDCl}_{3}$ )

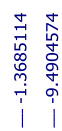
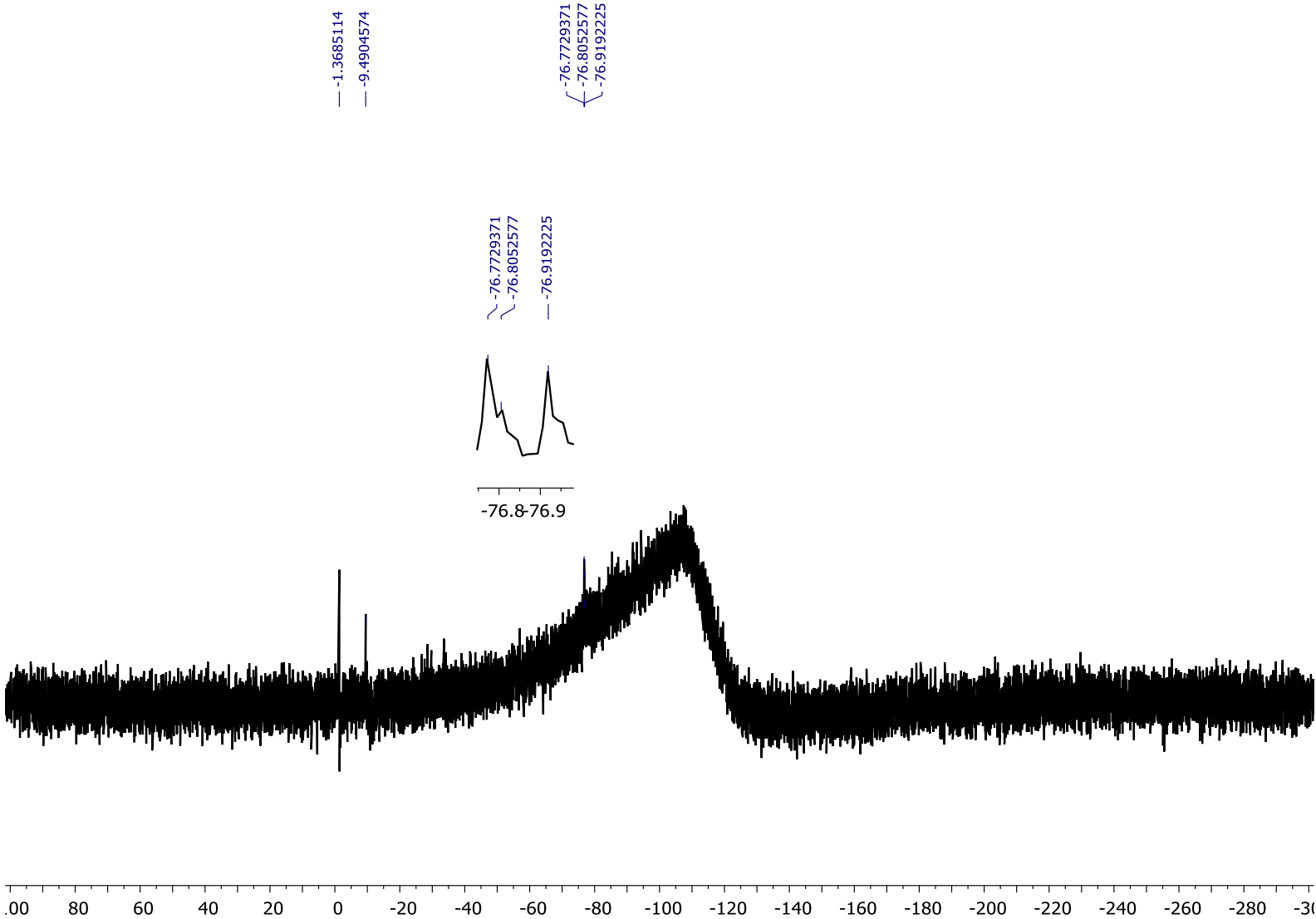
MALDI-TOF MS

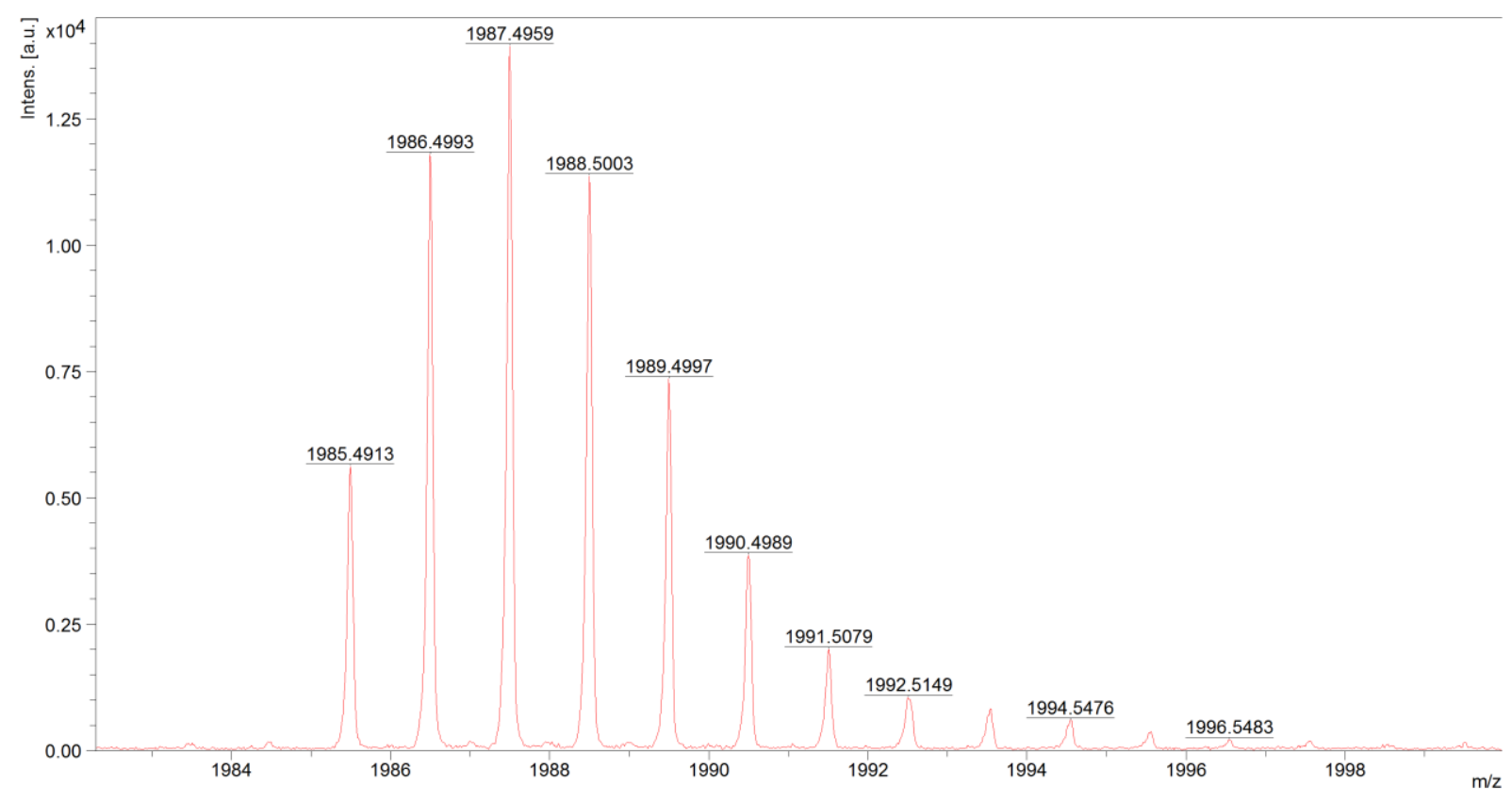


Compound 9a

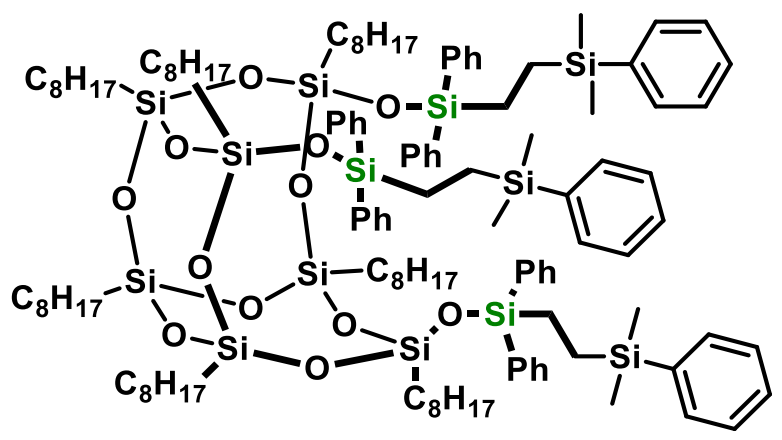

Chemical Formula: $\mathrm{C}_{122} \mathrm{H}_{194} \mathrm{O}_{12} \mathrm{Si}_{13}$

${ }^{1} \mathrm{H}$ NMR (300 MHz, Chloroform-d) $87.65-7.26(\mathrm{~m}, 36 \mathrm{H}), 7.22(\mathrm{~m}, 9 \mathrm{H}), 1.91-1.64(\mathrm{~m}, 7 \mathrm{H}), 1.36-0.67$ $(m, 112 \mathrm{H}), 0.63-0.42(m, 12 \mathrm{H}), 0.37--0.09(\mathrm{~m}, 18 \mathrm{H})$.

${ }^{13} \mathrm{C}$ NMR $\left(101 \mathrm{MHz}, \mathrm{CDCl}_{3}\right) \delta 139.32,134.70,134.65,134.61,134.58,134.39,134.33,133.83,133.81$, $130.01,129.98,129.60,129.58,129.24,129.05,128.95,128.91,128.88,128.06,127.95,127.91$, $127.85,127.81,55.22,54.90,54.72,54.67,54.63,54.40,54.08,31.31,31.29,31.23,30.35,30.29$, $25.32,25.15,24.57,24.05,23.65,7.68,7.66,7.46,7.26,7.23,7.10,7.08,7.06,-3.46$.

${ }^{29} \mathrm{Si} \mathrm{NMR}\left(79 \mathrm{MHz}, \mathrm{CDCl}_{3}\right)$ $\delta-0.96,-0.98,-1.05,-1.12,-1.16,-1.18,-66.74,-66.87,-67.17,-67.27,-68.19$, $-68.23$.

MALDI-TOF MS (m/z): calcd. for $\mathrm{C}_{122} \mathrm{H}_{194} \mathrm{O}_{12} \mathrm{Si}_{13} \mathrm{Na} 1240,98$; found 1240,20.

${ }^{1} \mathrm{H}$ NMR (300 MHz, $\mathrm{CDCl}_{3}$ )

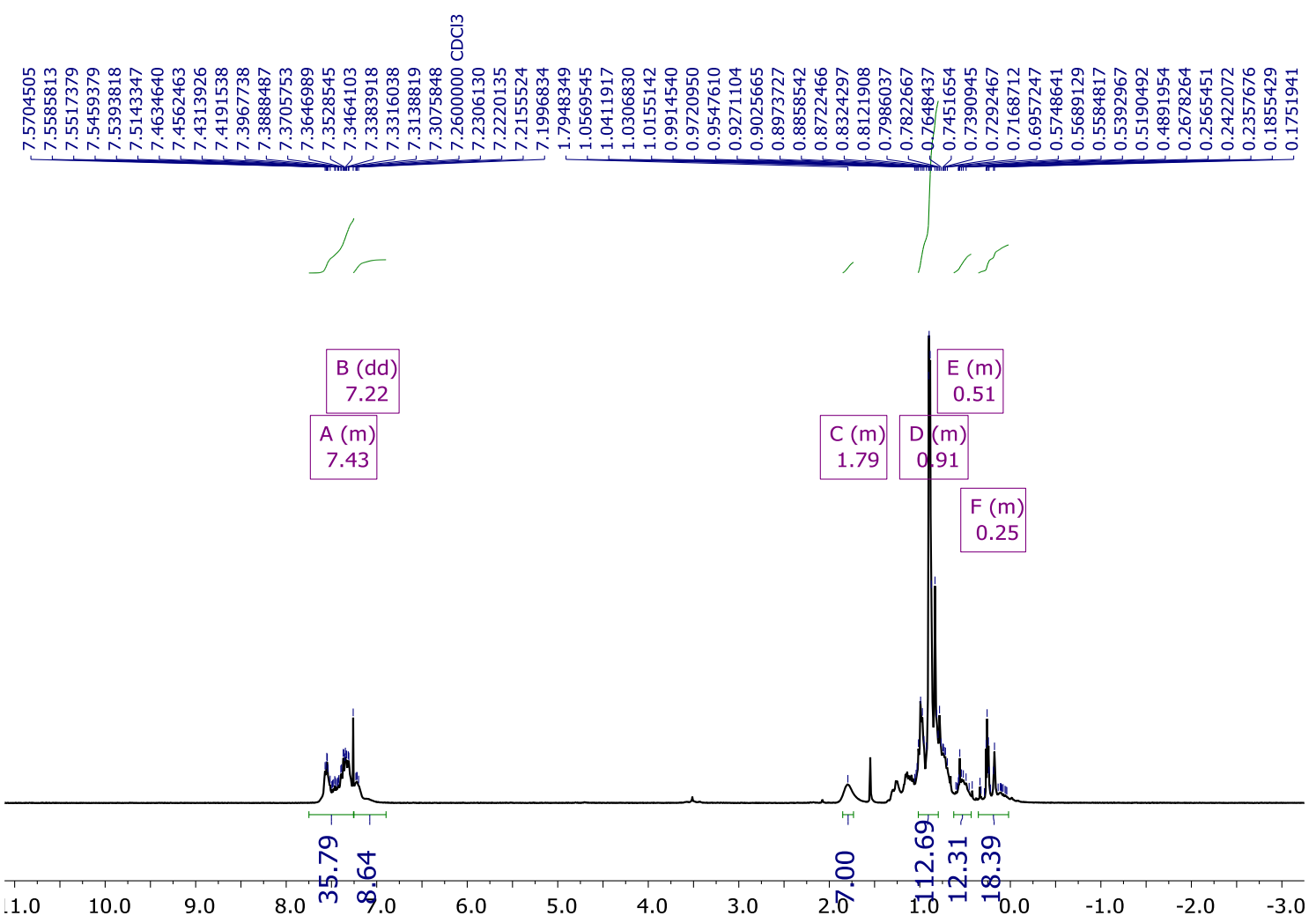


$\left.{ }^{13} \mathrm{C} \mathrm{NMR} \mathrm{(101} \mathrm{MHz,} \mathrm{CDCl}_{3}\right)$
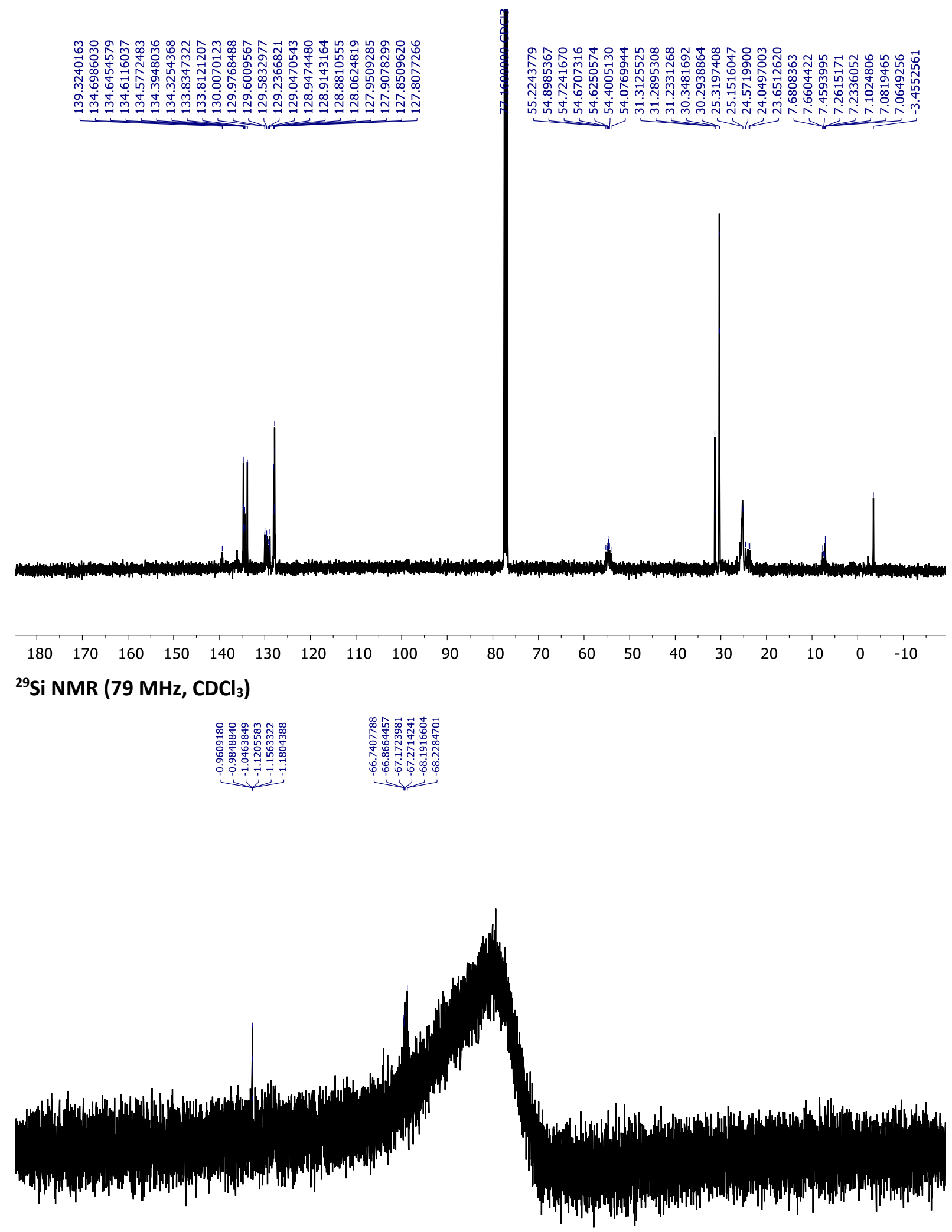

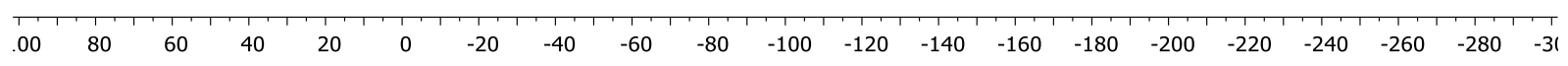


MALDI-TOF MS

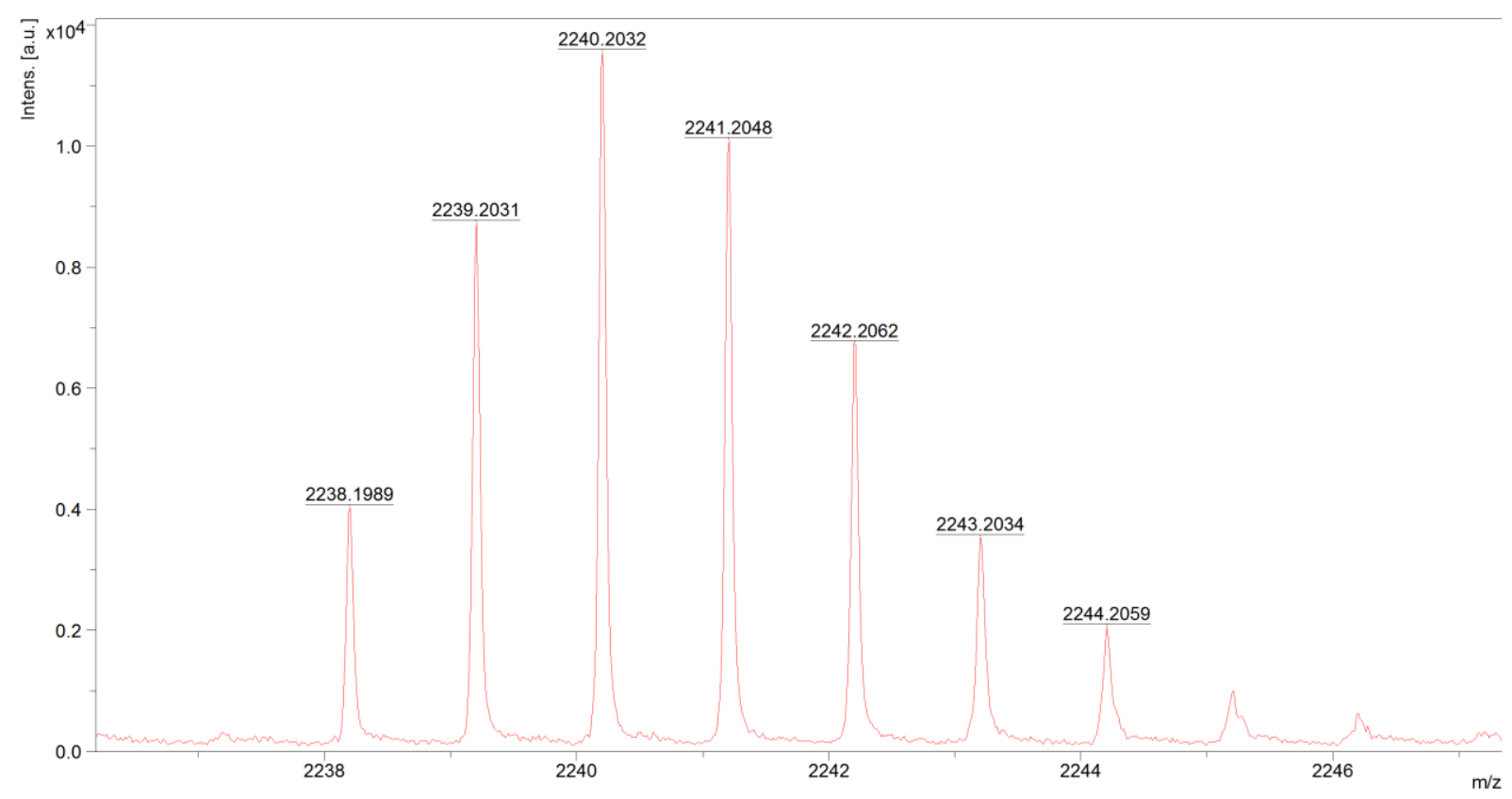


Compound $1 b$

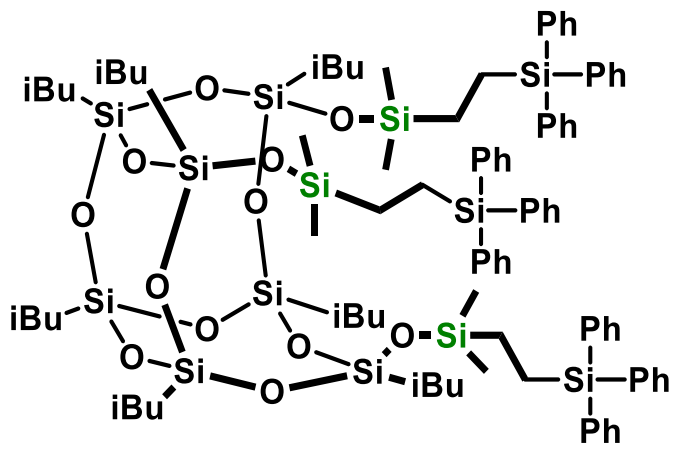

Chemical Formula: $\mathrm{C}_{94} \mathrm{H}_{138} \mathrm{O}_{12} \mathrm{Si}_{13}$

${ }^{1} \mathrm{H}$ NMR (300 MHz, Chloroform-d) $\delta 7.68-7.45(\mathrm{~m}, 17 \mathrm{H}), 7.45-7.28(\mathrm{~m}, 26 \mathrm{H}), 7.26-7.16(\mathrm{~m}, 2 \mathrm{H})$, $1.91-1.72(\mathrm{~m}, 7 \mathrm{H}), 1.36-1.21(\mathrm{~m}, 4 \mathrm{H}), 1.01-0.84(\mathrm{~m}, 42 \mathrm{H}), 0.66-0.47(\mathrm{~m}, 22 \mathrm{H}), 0.20--0.02(\mathrm{~m}$, $18 \mathrm{H})$.

${ }^{13} \mathrm{C}$ NMR $\left(75 \mathrm{MHz}, \mathrm{CDCl}_{3}\right) \delta$ 135.86, 135.45, 129.43, 127.94, 26.19, 25.99, 25.78, 25.21, 24.24, 24.10, $23.87,22.60,10.50,4.76,-0.20$.

${ }^{29} \mathrm{Si}$ NMR $\left(79 \mathrm{MHz}, \mathrm{CDCl}_{3}\right)$ $\delta 9.65,-9.41,-67.13,-67.29,-67.69,-67.74,-67.76,-67.95,-68.00,-68.70,-$ 69.12.

MALDI-TOF MS (m/z): calcd. for $\mathrm{C}_{94} \mathrm{H}_{138} \mathrm{O}_{12} \mathrm{Si}_{13} \mathrm{Na}$ 1848,22; found 1847,71.

${ }^{1} \mathrm{H}$ NMR (300 MHz, $\mathrm{CDCl}_{3}$ )
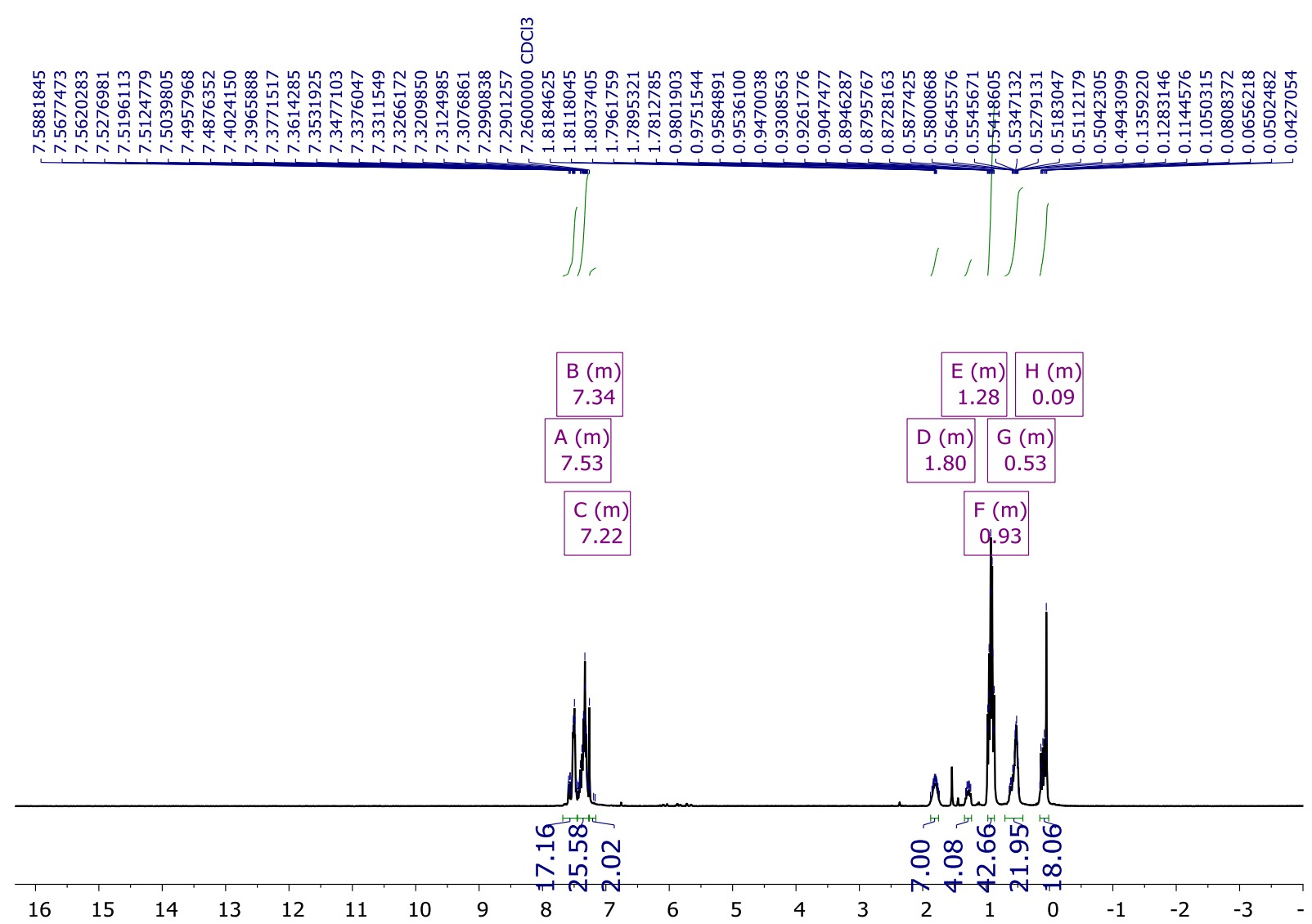
${ }^{13} \mathrm{C}$ NMR (101 MHz, $\mathrm{CDCl}_{3}$ )
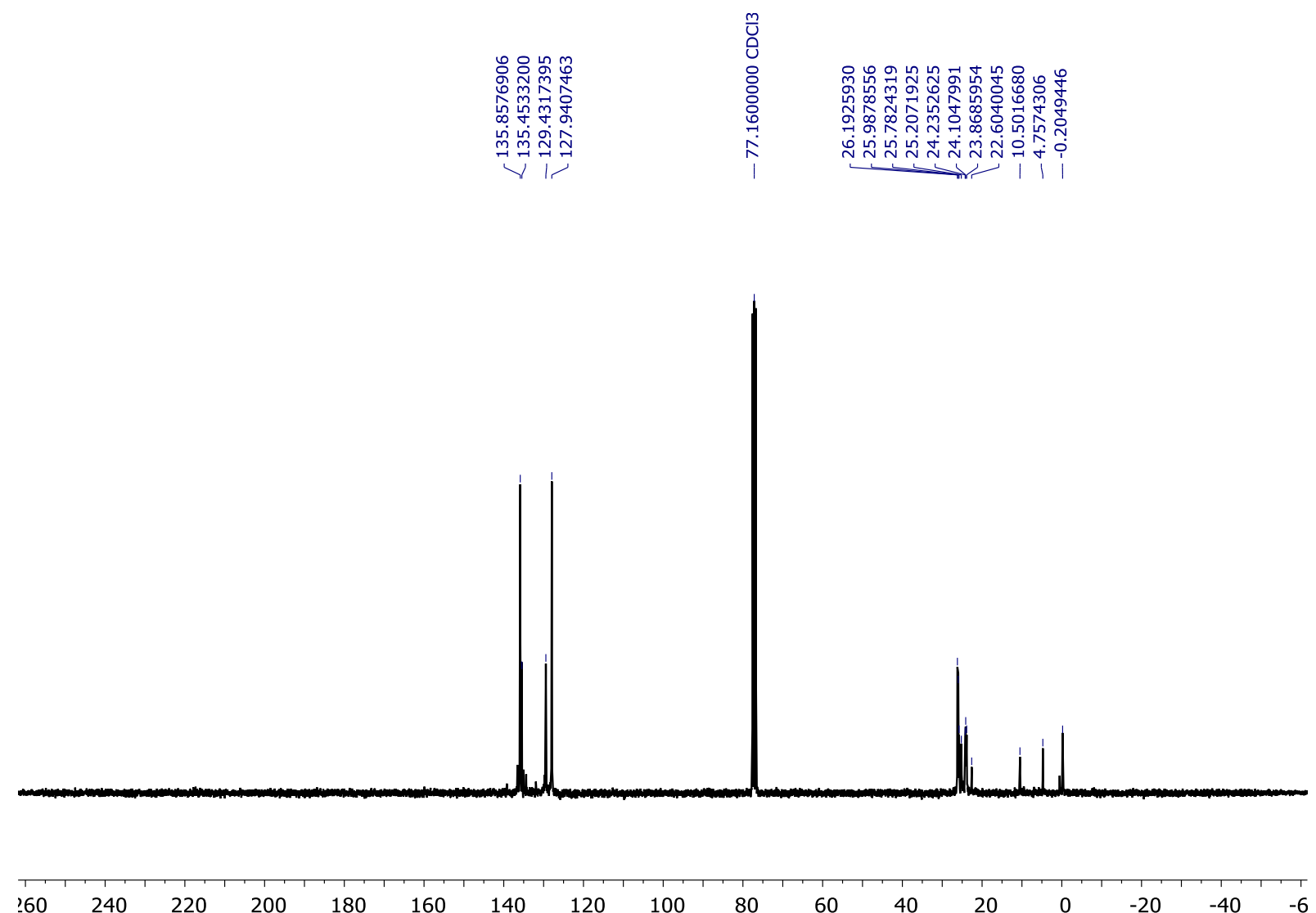

${ }^{29} \mathrm{Si}$ NMR (79 MHz, $\mathrm{CDCl}_{3}$ )
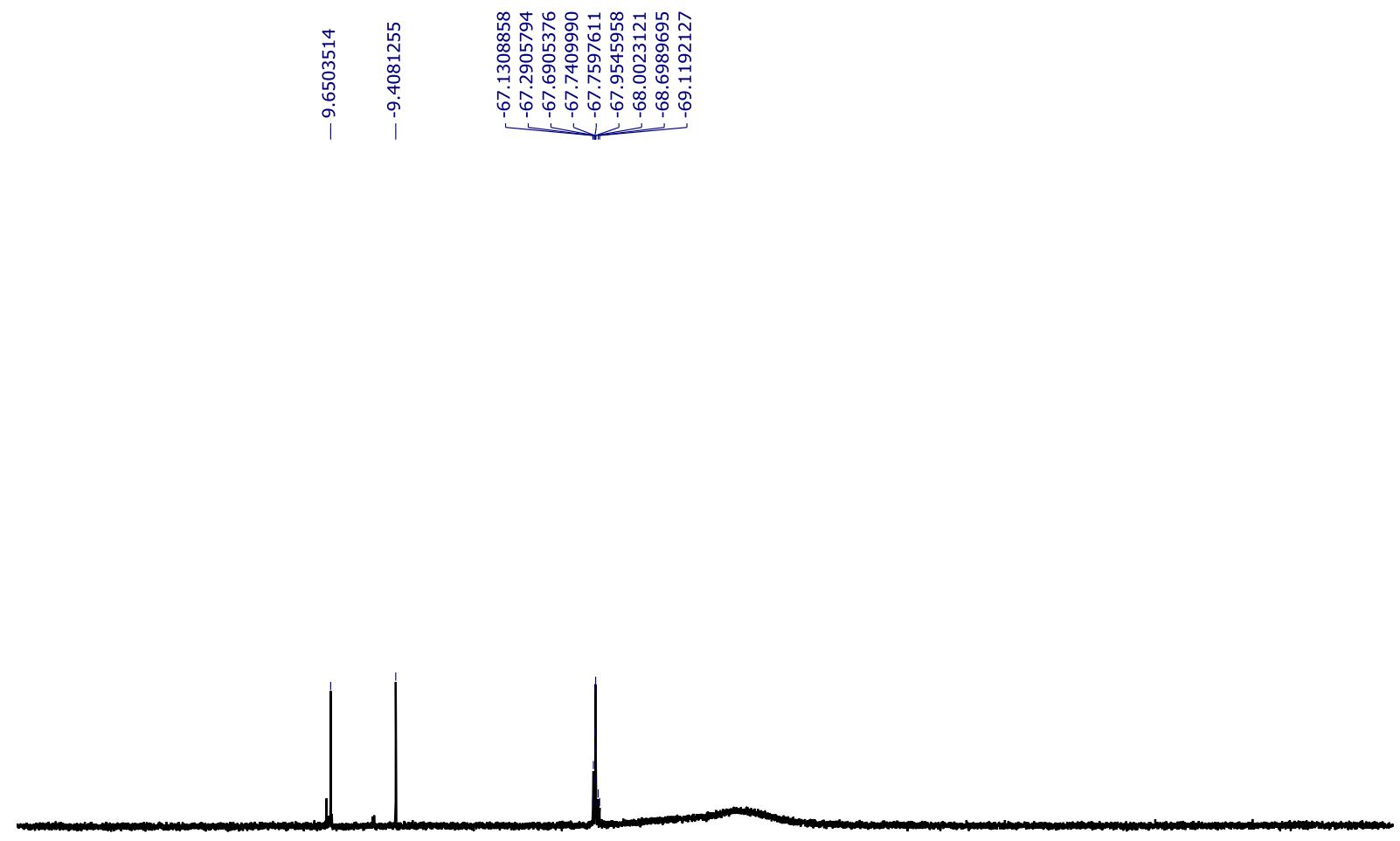

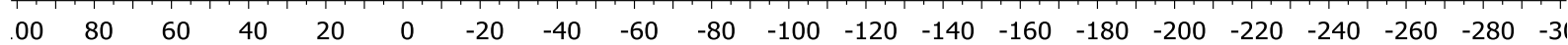


MALDI-TOF MS

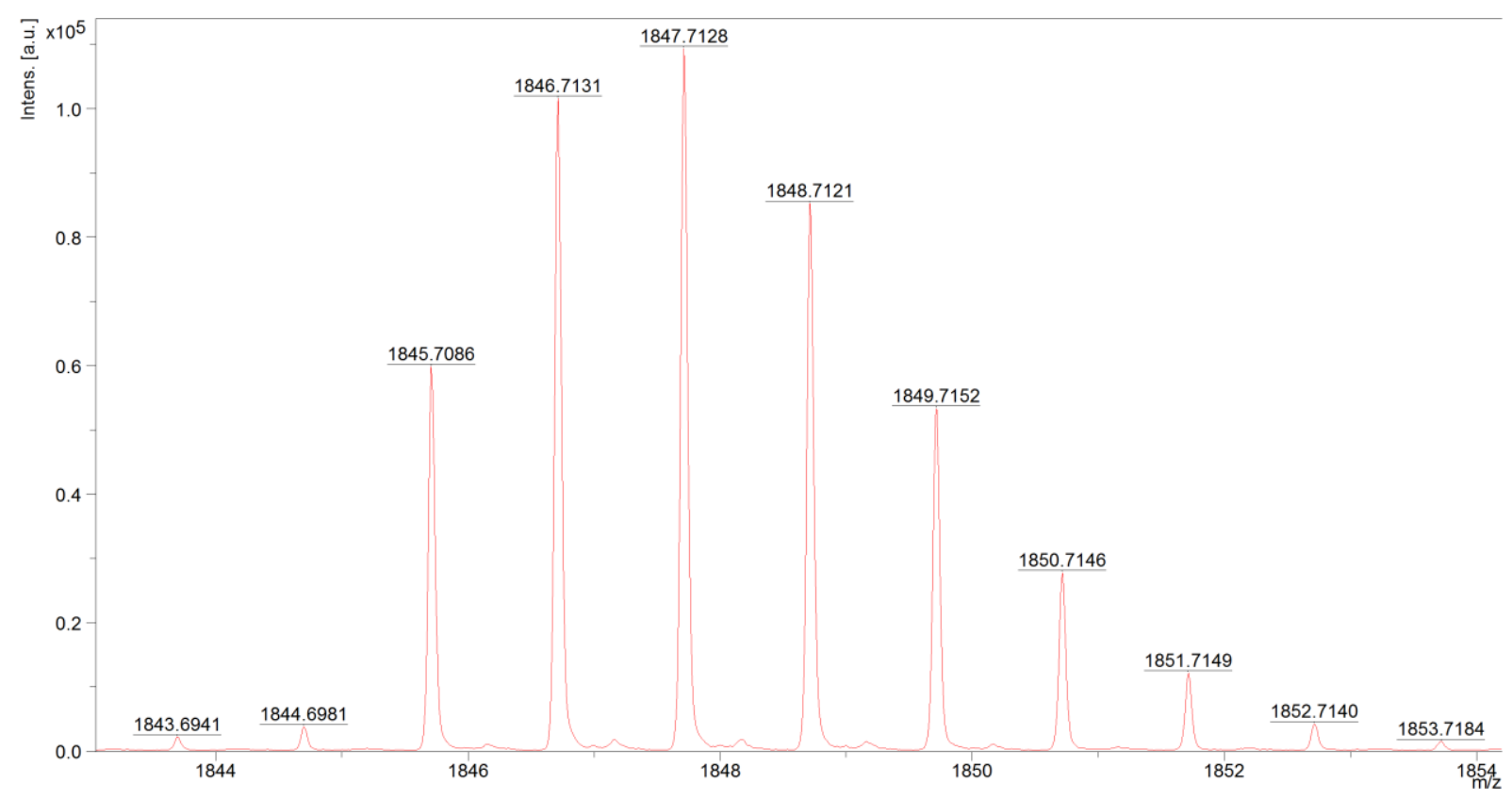


Compound $2 b$

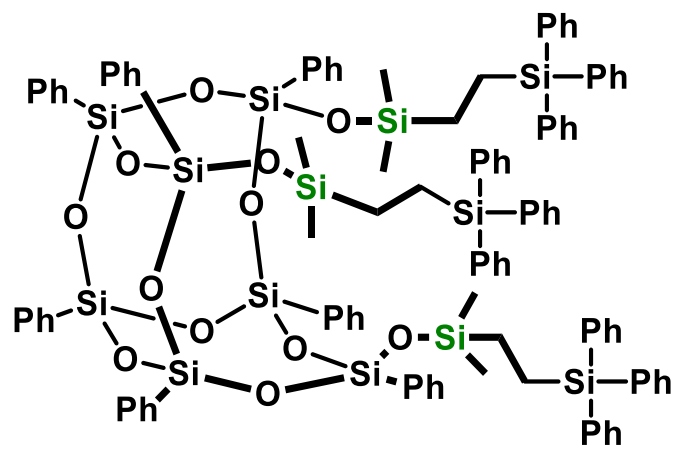

Chemical Formula: $\mathrm{C}_{108} \mathrm{H}_{110} \mathrm{O}_{12} \mathrm{Si}_{13}$

${ }^{1} \mathrm{H}$ NMR (300 MHz, Chloroform-d) $\delta 7.40-7.26(\mathrm{~m}, 38 \mathrm{H}), 7.25-7.15(\mathrm{~m}, 42 \mathrm{H}), 1.27-1.06(\mathrm{~m}, 6 \mathrm{H})$, $0.66-0.49(\mathrm{~m}, 6 \mathrm{H}), 0.24-0.05(\mathrm{~m}, 18 \mathrm{H})$.

${ }^{13} \mathrm{C}$ NMR $\left(101 \mathrm{MHz}, \mathrm{CDCl}_{3}\right) \delta 136.55,135.82,135.31,134.12,134.07,132.95,131.25,130.19,130.04$, $129.74,129.37,128.01,127.90,127.68,127.64,10.44,4.64,-0.15$.

${ }^{29} \mathrm{Si}$ NMR $\left(79 \mathrm{MHz}, \mathrm{CDCl}_{3}\right) \delta 12.13,-9.51,-77.81,-78.17$.

MALDI-TOF MS (m/z): calcd. for $\mathrm{C}_{108} \mathrm{H}_{110} \mathrm{O}_{12} \mathrm{Si}_{13} \mathrm{Na} 1988,15$; found 1987,48.

\section{${ }^{1} \mathrm{H}$ NMR $\left(300 \mathrm{MHz}, \mathrm{CDCl}_{3}\right)$}
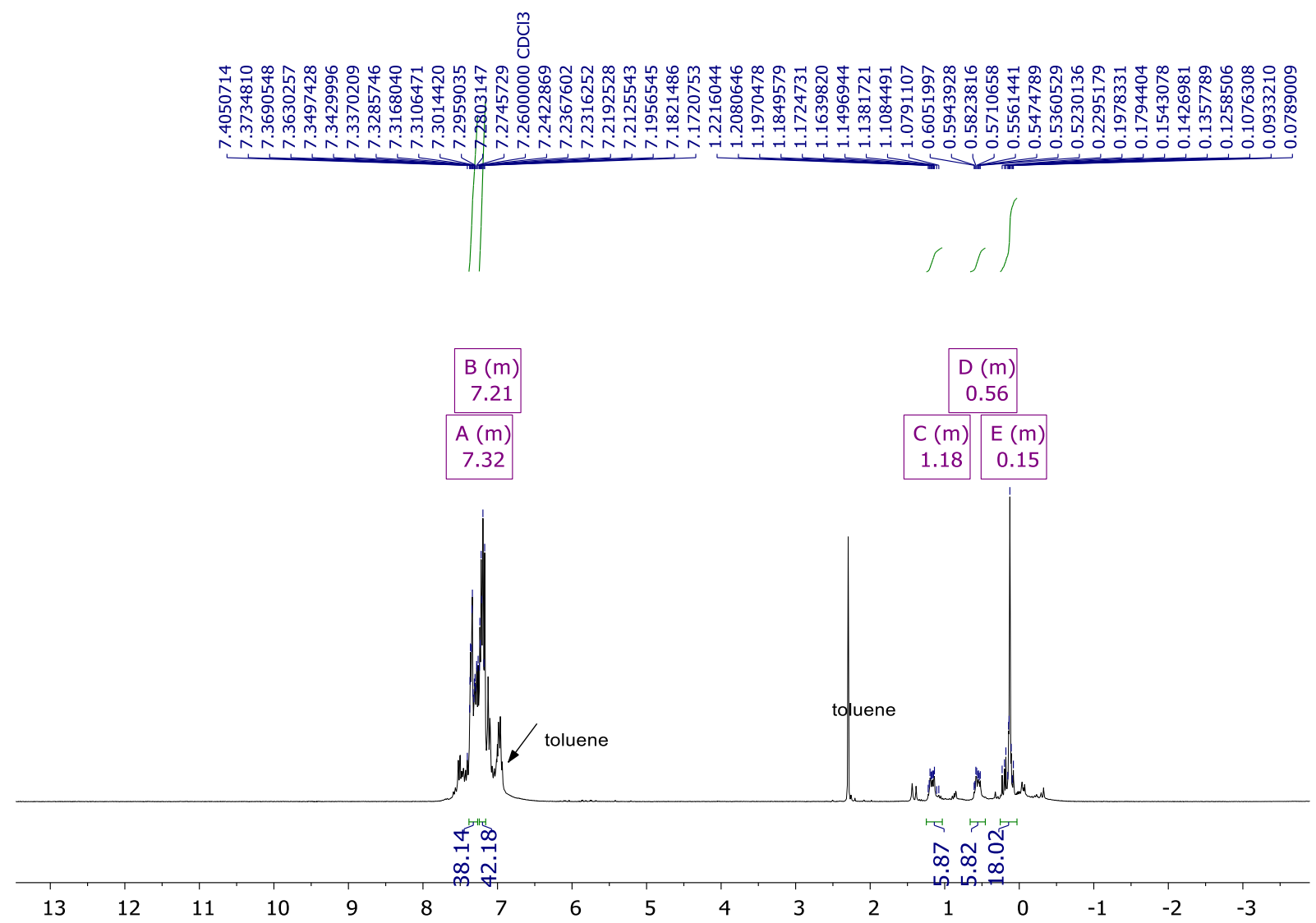
${ }^{13} \mathrm{C}$ NMR (101 MHz, $\left.\mathrm{CDCl}_{3}\right)$
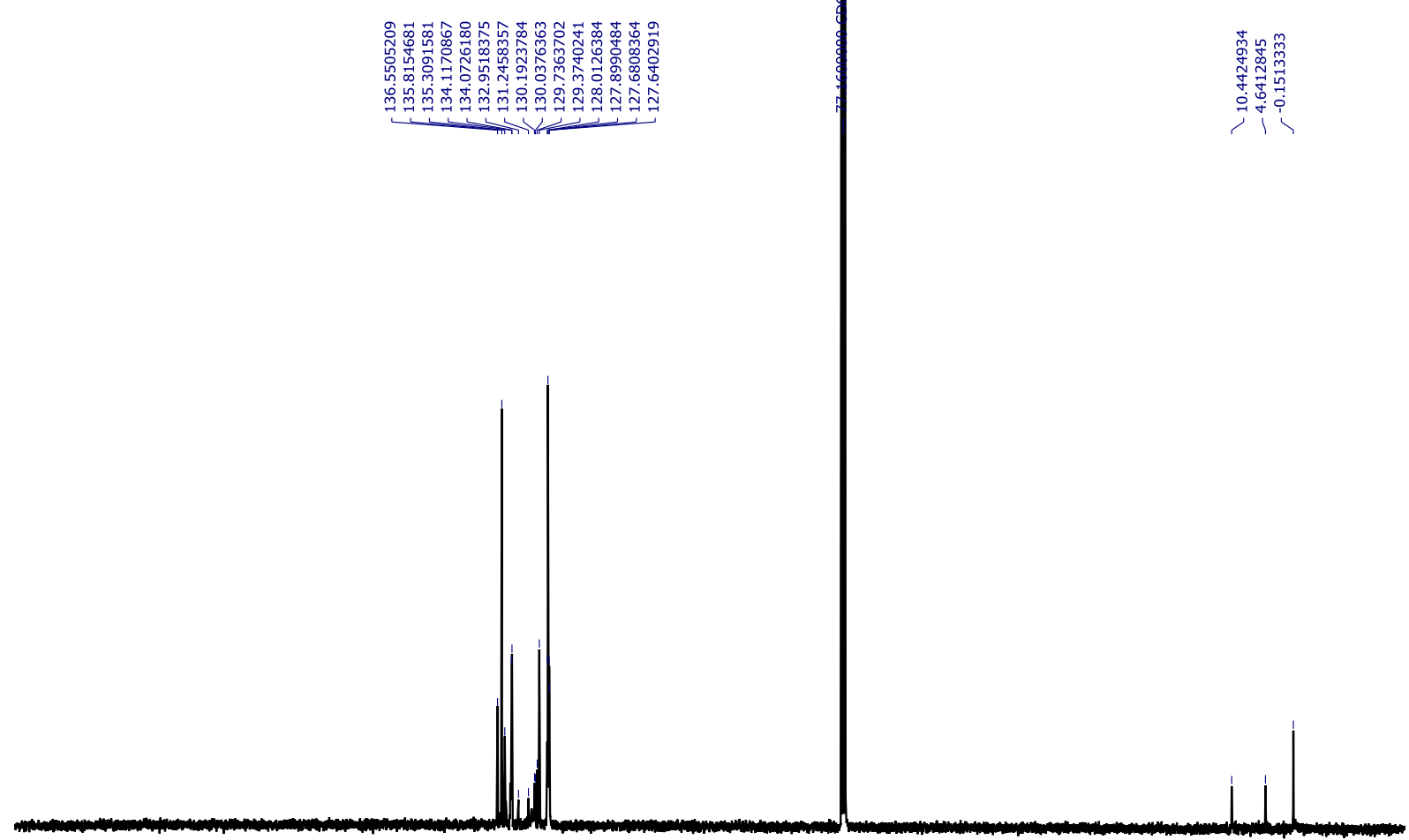

$\begin{array}{lllllllllllllllllllllll}210 & 200 & 190 & 180 & 170 & 160 & 150 & 140 & 130 & 120 & 110 & 100 & 90 & 80 & 70 & 60 & 50 & 40 & 30 & 20 & 10 & 0 & -10\end{array}$

${ }^{29} \mathrm{Si}$ NMR $\left(79 \mathrm{MHz}, \mathrm{CDCl}_{3}\right)$
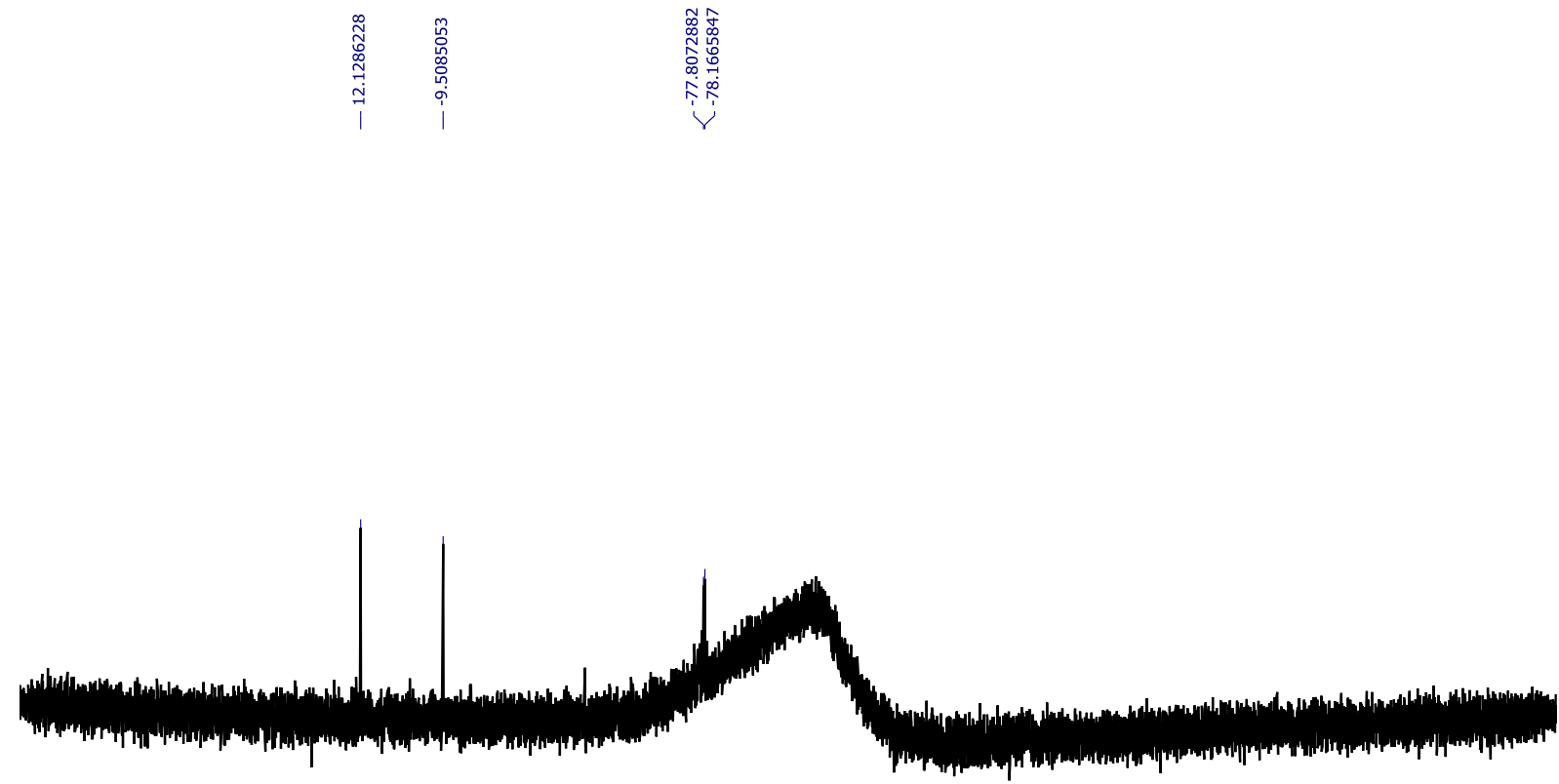
MALDI-TOF MS

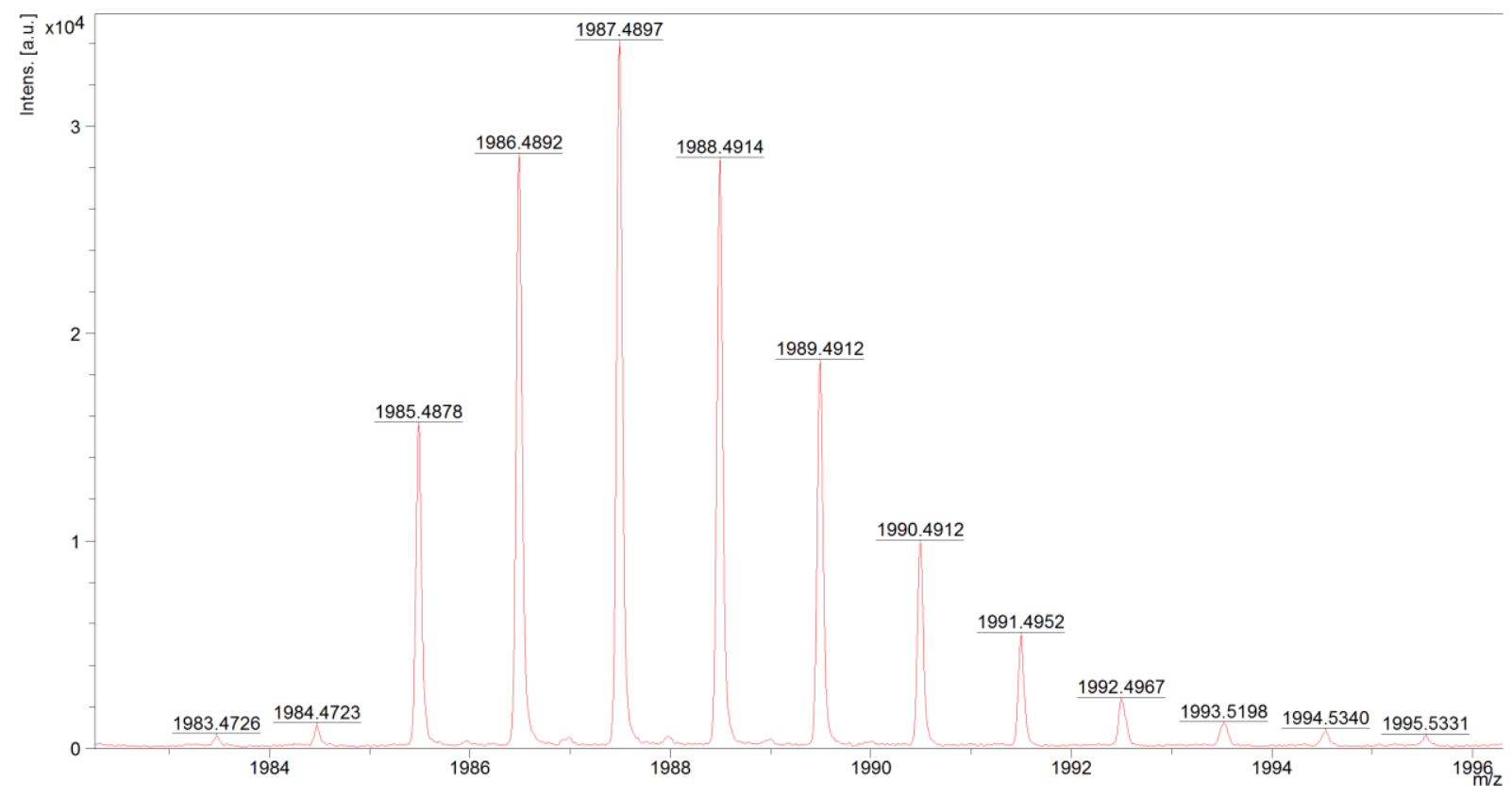


Compound $4 b$

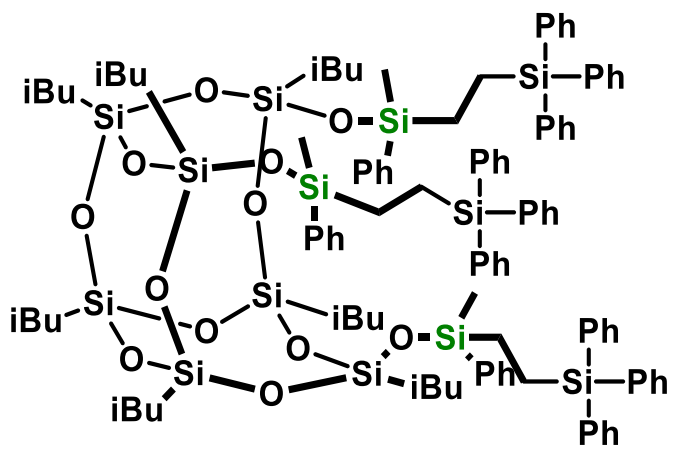

Chemical Formula: $\mathrm{C}_{109} \mathrm{H}_{144} \mathrm{O}_{12} \mathrm{Si}_{13}$

${ }^{1} \mathrm{H}$ NMR (300 MHz, Chloroform-d) $\delta 7.67-7.27(\mathrm{~m}, 50 \mathrm{H}), 7.25-7.09(\mathrm{~m}, 10 \mathrm{H}), 1.96-1.64(\mathrm{~m}, 7 \mathrm{H})$, $1.31-1.08(\mathrm{~m}, 4 \mathrm{H}), 1.02-0.78(\mathrm{~m}, 48 \mathrm{H}), 0.67-0.42(\mathrm{~m}, 16 \mathrm{H}), 0.35--0.06(\mathrm{~m}, 9 \mathrm{H})$.

${ }^{13} \mathrm{C}$ NMR $\left(101 \mathrm{MHz}, \mathrm{CDCl}_{3}\right) \delta 136.55,135.89,135.83,135.33,135.28,133.69,133.66,129.74,129.49$, $129.43,128.02,127.97,127.91,127.66,127.62,26.22$, 26.18, 26.16, 26.02, 25.96, 25.91, 25.88, 25.80, $25.77,25.19,24.26,24.17,24.13,24.04,23.97,23.83,22.58,9.22,4.50,-1.87$.

${ }^{29} \mathrm{Si}$ NMR $\left(79 \mathrm{MHz}, \mathrm{CDCl}_{3}\right) \delta-0.36,-0.49,-0.54,-9.40,-67.25,-67.32,-67.37,-67.40,-67.41,-67.47,-$ $67.52,-67.57,-67.59$.

MALDI-TOF MS (m/z): calcd. for $\mathrm{C}_{109} \mathrm{H}_{144} \mathrm{O}_{12} \mathrm{Si}_{13} \mathrm{Na} 2034,43$; found 2033,75.

${ }^{1} \mathrm{H}$ NMR (300 MHz, $\left.\mathrm{CDCl}_{3}\right)$

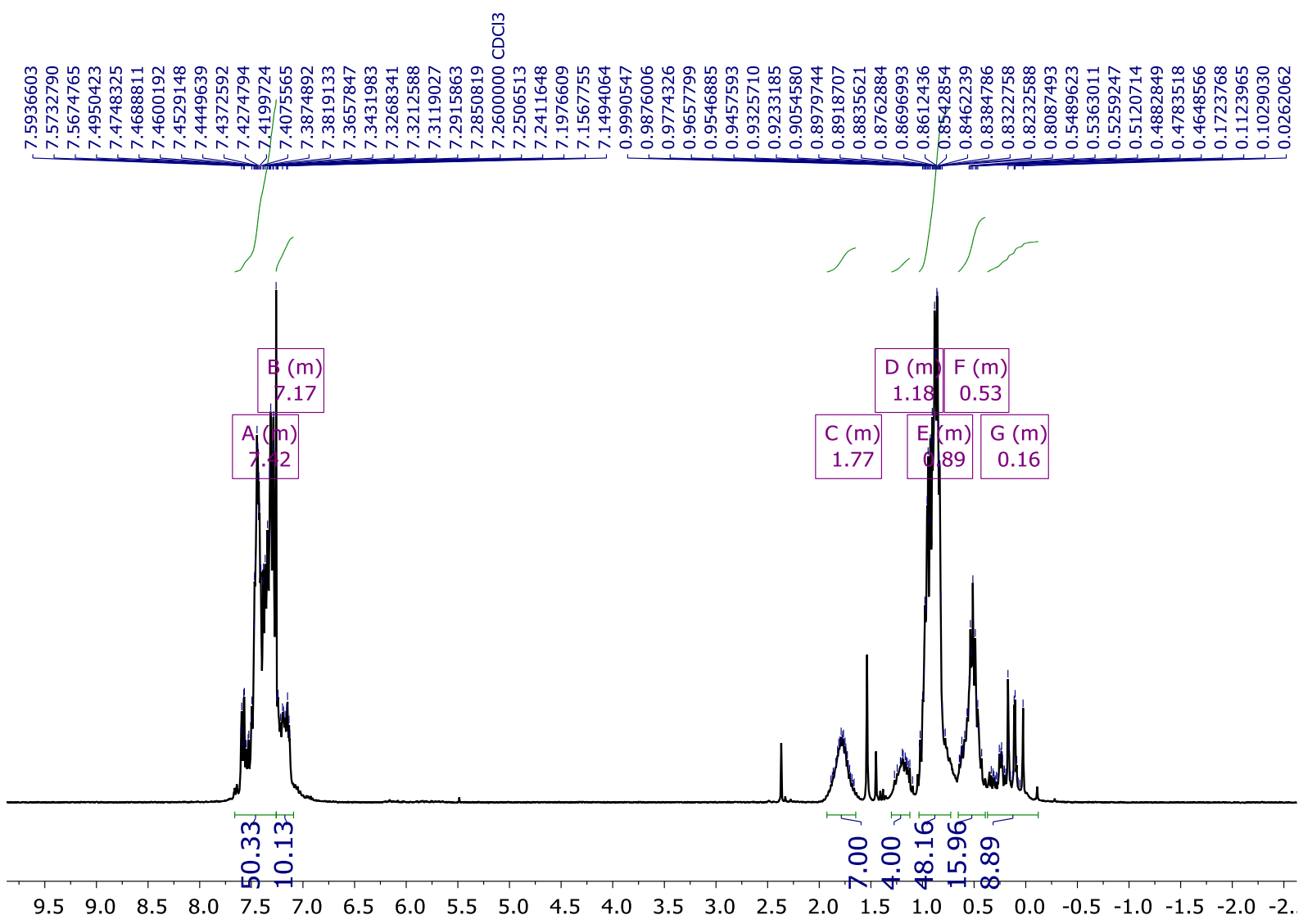




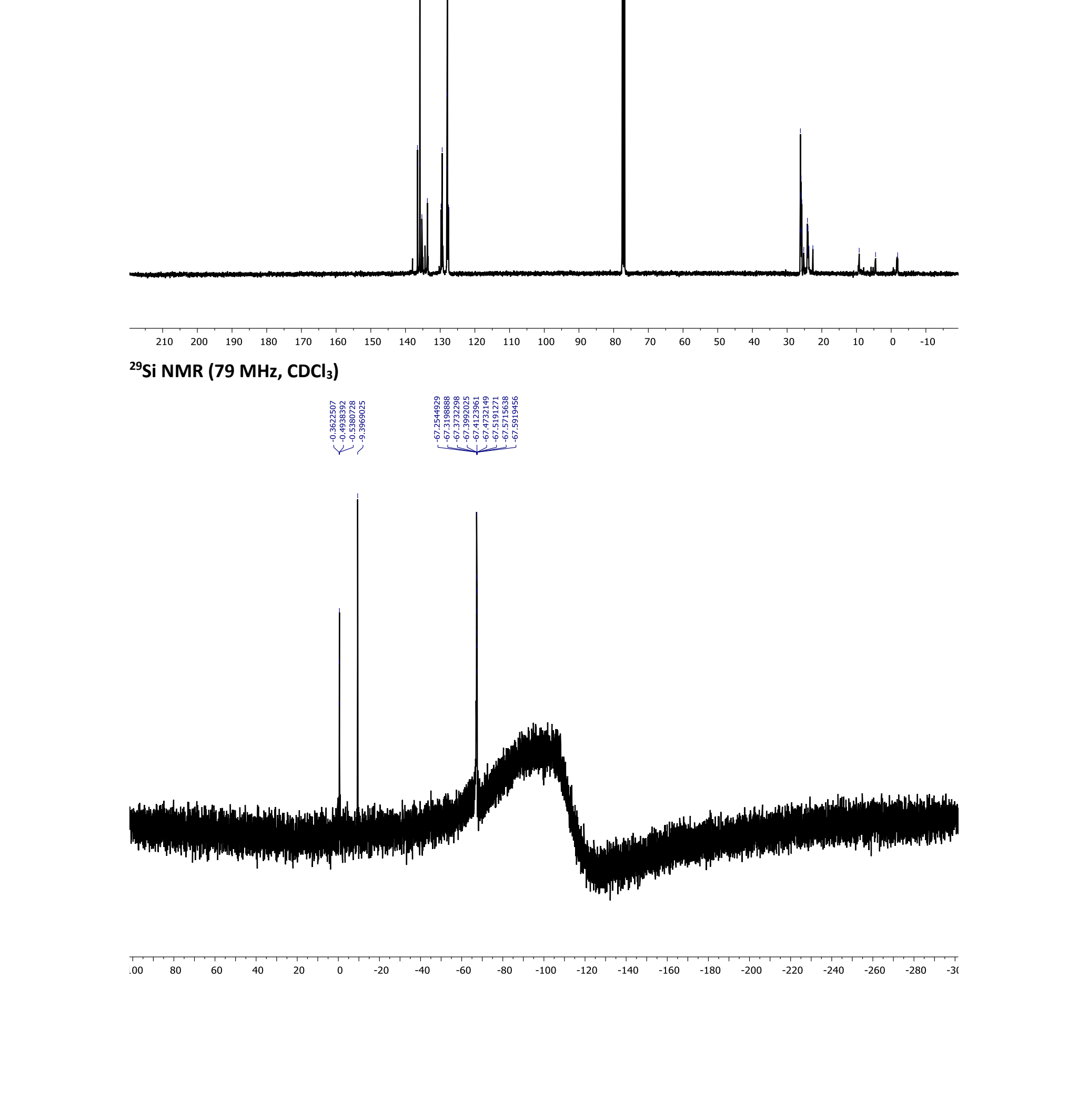


MALDI-TOF MS

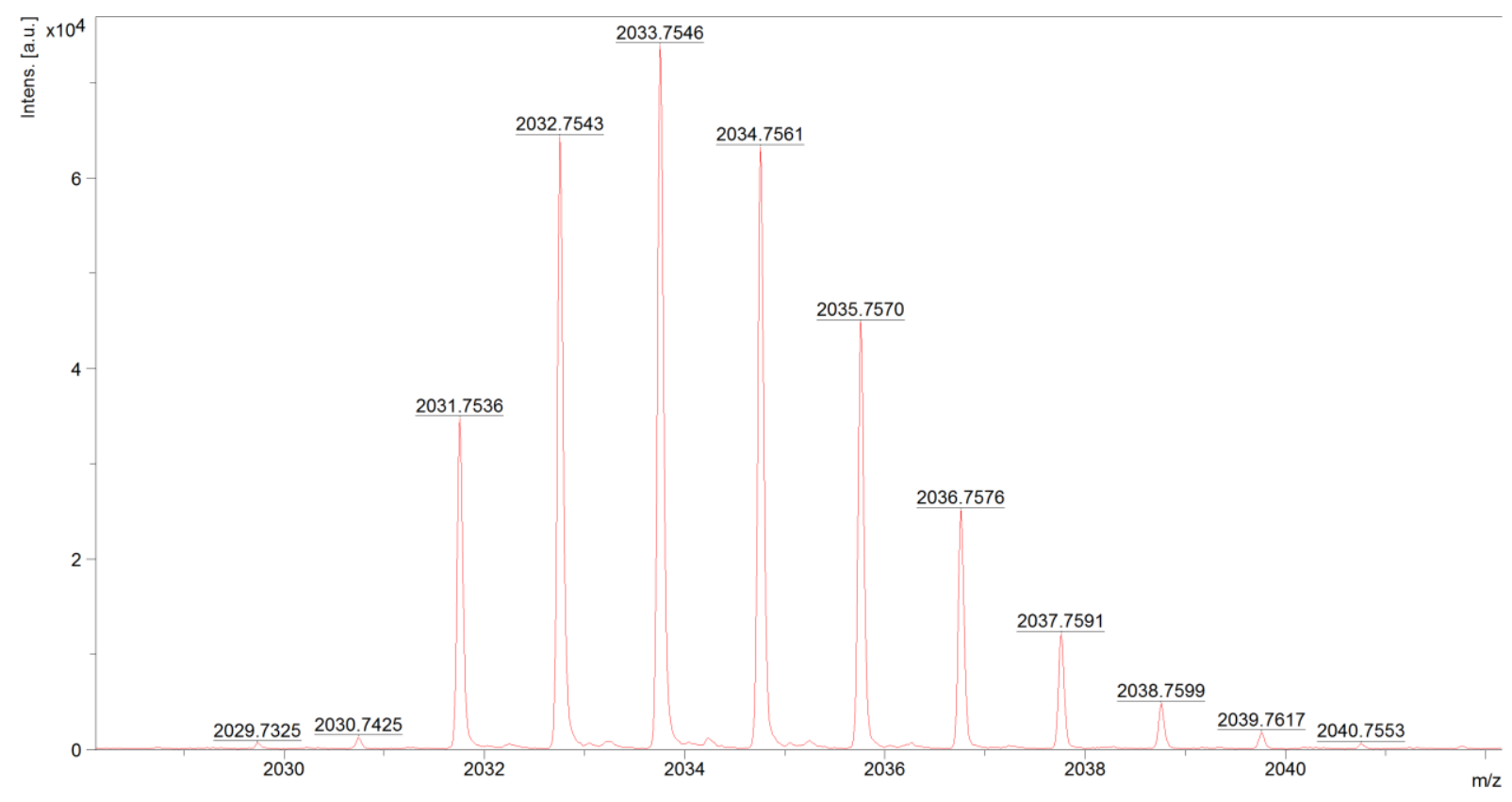


Compound 7b

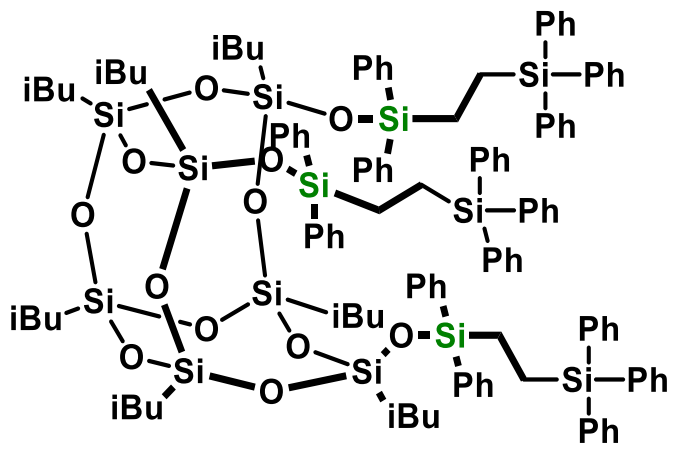

Chemical Formula: $\mathrm{C}_{124} \mathrm{H}_{150} \mathrm{O}_{12} \mathrm{Si}_{13}$

${ }^{1} \mathrm{H}$ NMR (300 MHz, Chloroform-d) $\delta 7.70-6.89(\mathrm{~m}, 75 \mathrm{H}), 1.93-1.60(\mathrm{~m}, 7 \mathrm{H}), 1.39-1.25(\mathrm{~m}, 4 \mathrm{H}), 1.18$ $-1.05(\mathrm{~m}, 4 \mathrm{H}), 1.03-0.73(\mathrm{~m}, 46 \mathrm{H}), 0.63-0.38(\mathrm{~m}, 18 \mathrm{H})$.

${ }^{13} \mathrm{C}$ NMR $\left(75 \mathrm{MHz}, \mathrm{CDCl}_{3}\right) \delta 135.90,135.83,135.75,135.72,135.13,134.85,134.51,129.53,129.43$, $127.94,127.78,127.62,26.15,26.06,25.95,25.80,25.54,25.12,24.00,23.86,22.63,8.05,4.62$.

${ }^{29} \mathrm{Si} \mathrm{NMR}\left(79 \mathrm{MHz}, \mathrm{CDCl}_{3}\right) \delta-9.38,-11.83,-66.02,-66.79,-67.32,-67.46,-67.56$.

MALDI-TOF MS (m/z): calcd. for $\mathrm{C}_{124} \mathrm{H}_{150} \mathrm{O}_{12} \mathrm{Si}_{13} \mathrm{Na} 2220,65$; found 2219,81.

\section{${ }^{1} \mathrm{H}$ NMR $\left(300 \mathrm{MHz}, \mathrm{CDCl}_{3}\right)$}

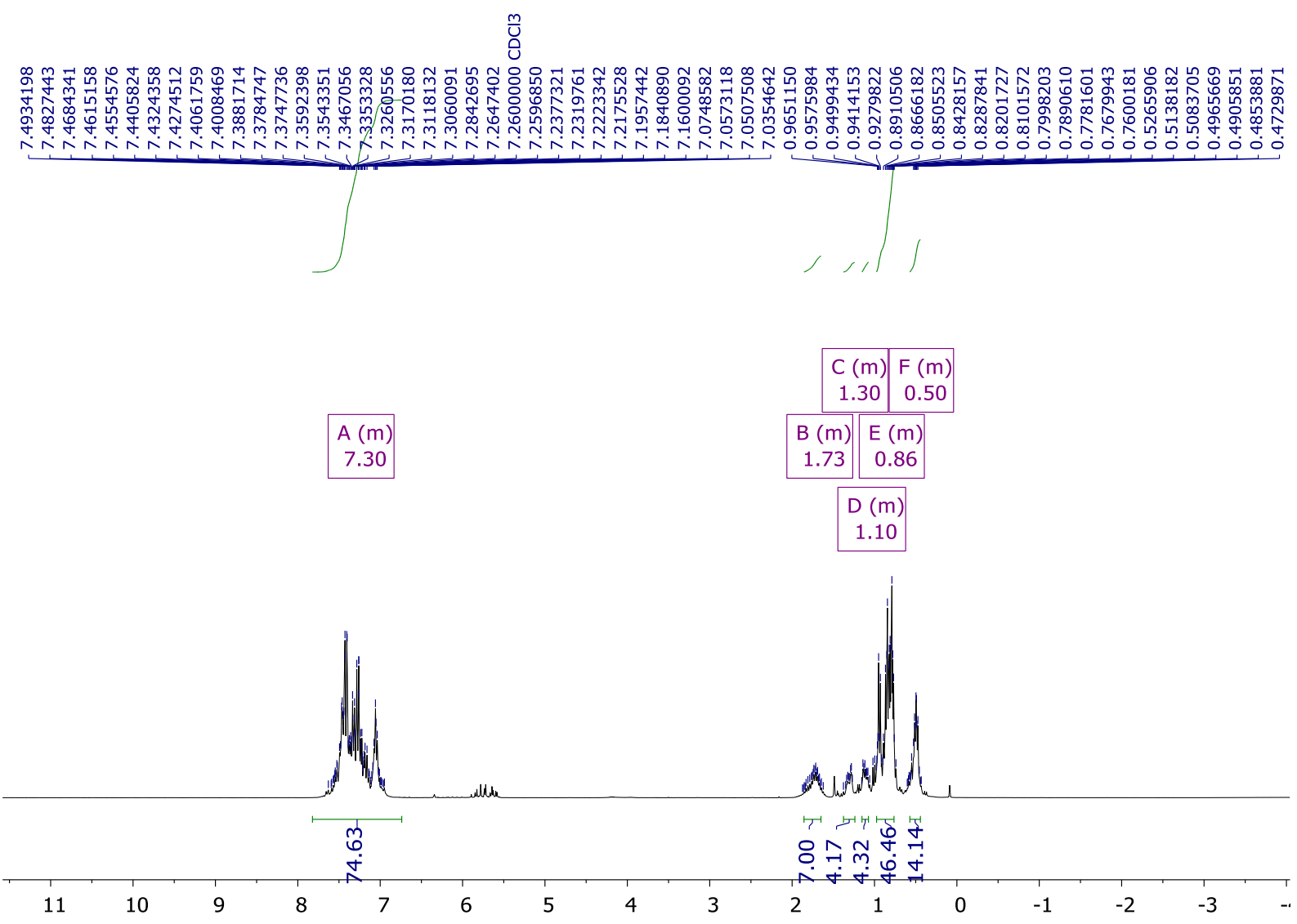


${ }^{13} \mathrm{C}$ NMR (101 MHz, $\mathrm{CDCl}_{3}$ )

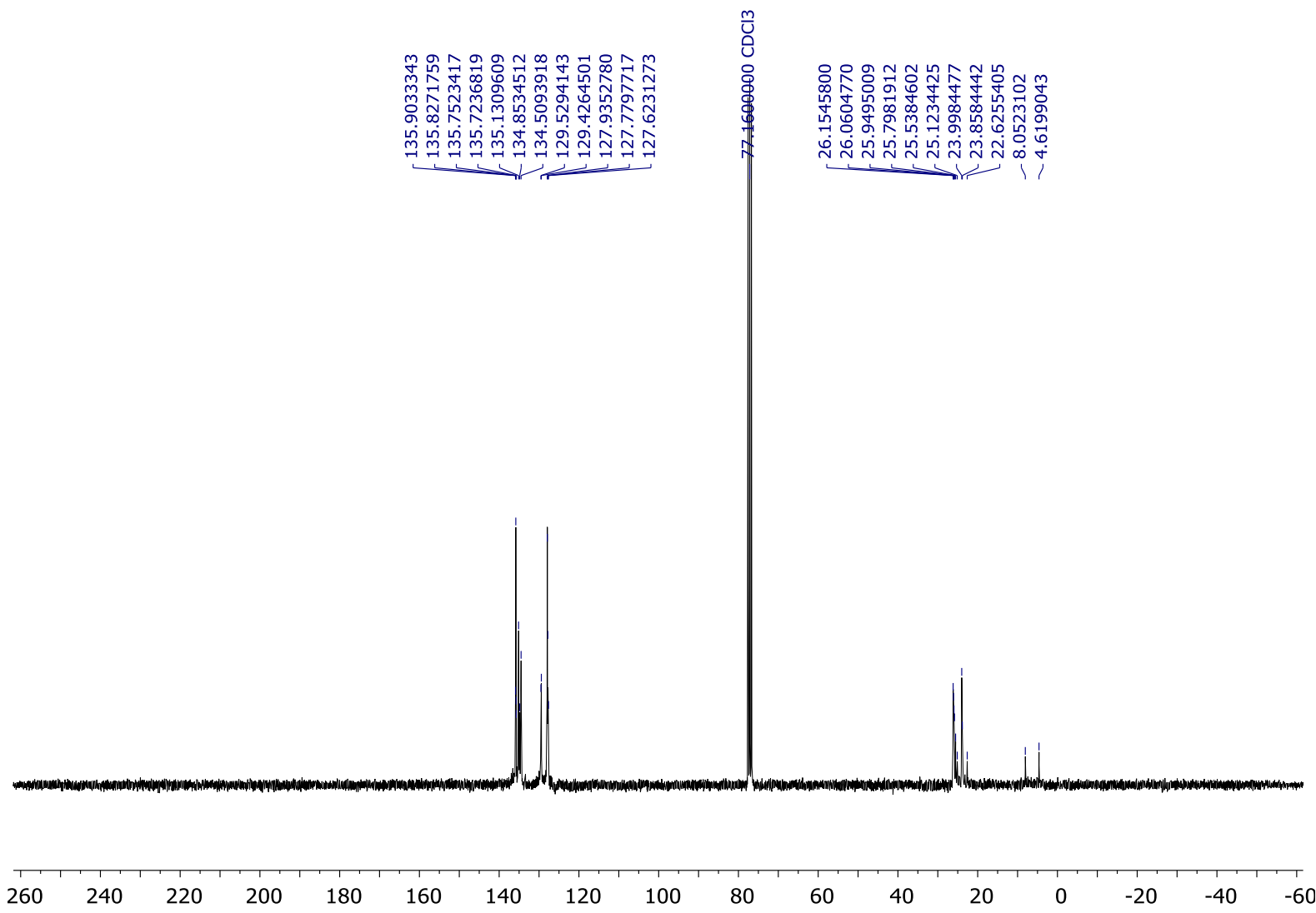

${ }^{29} \mathrm{Si}$ NMR $\left(79 \mathrm{MHz}, \mathrm{CDCl}_{3}\right.$ )
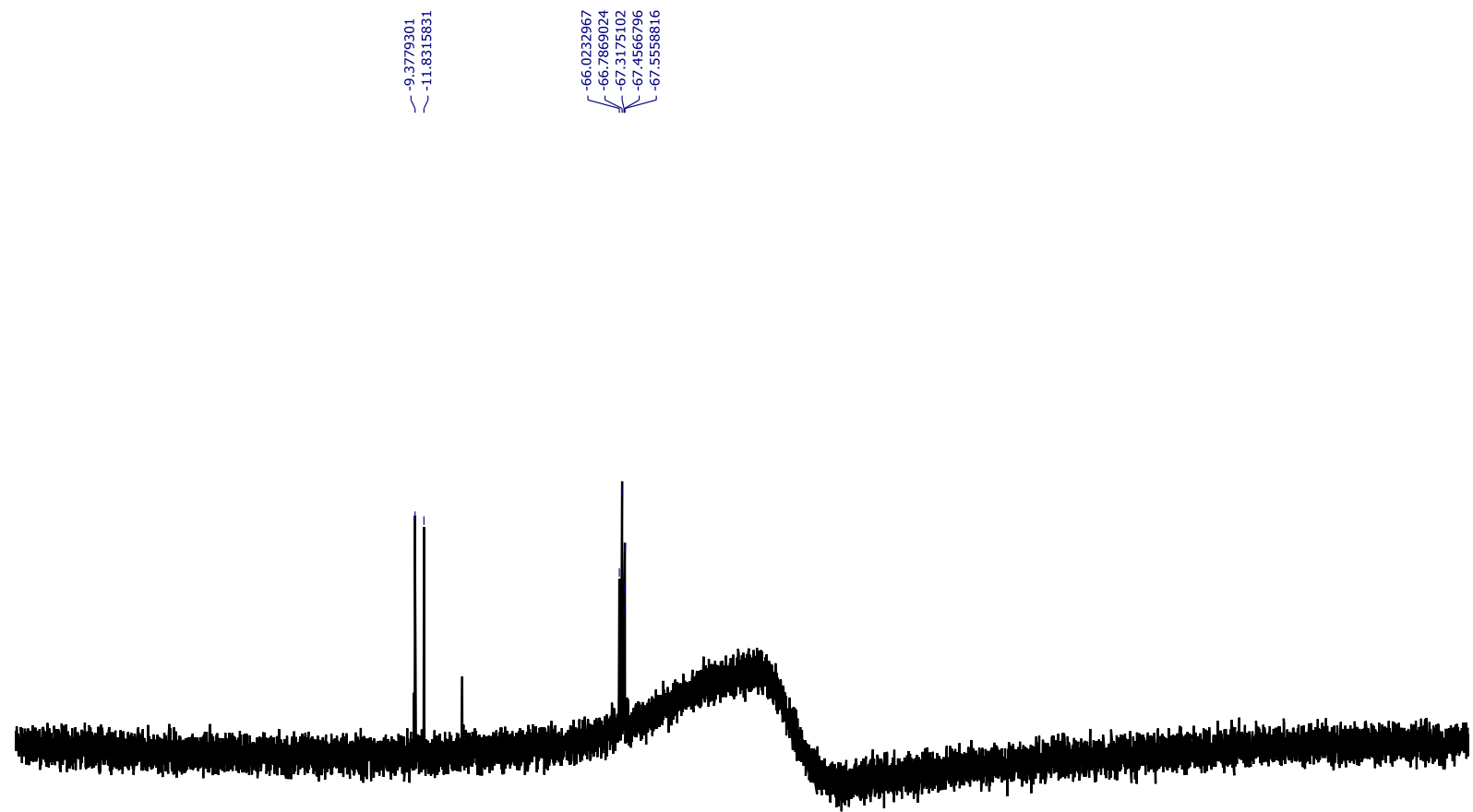

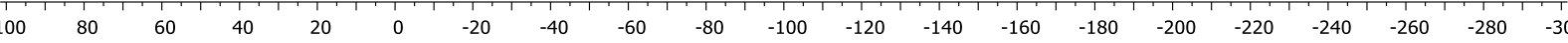


MALDI-TOF MS

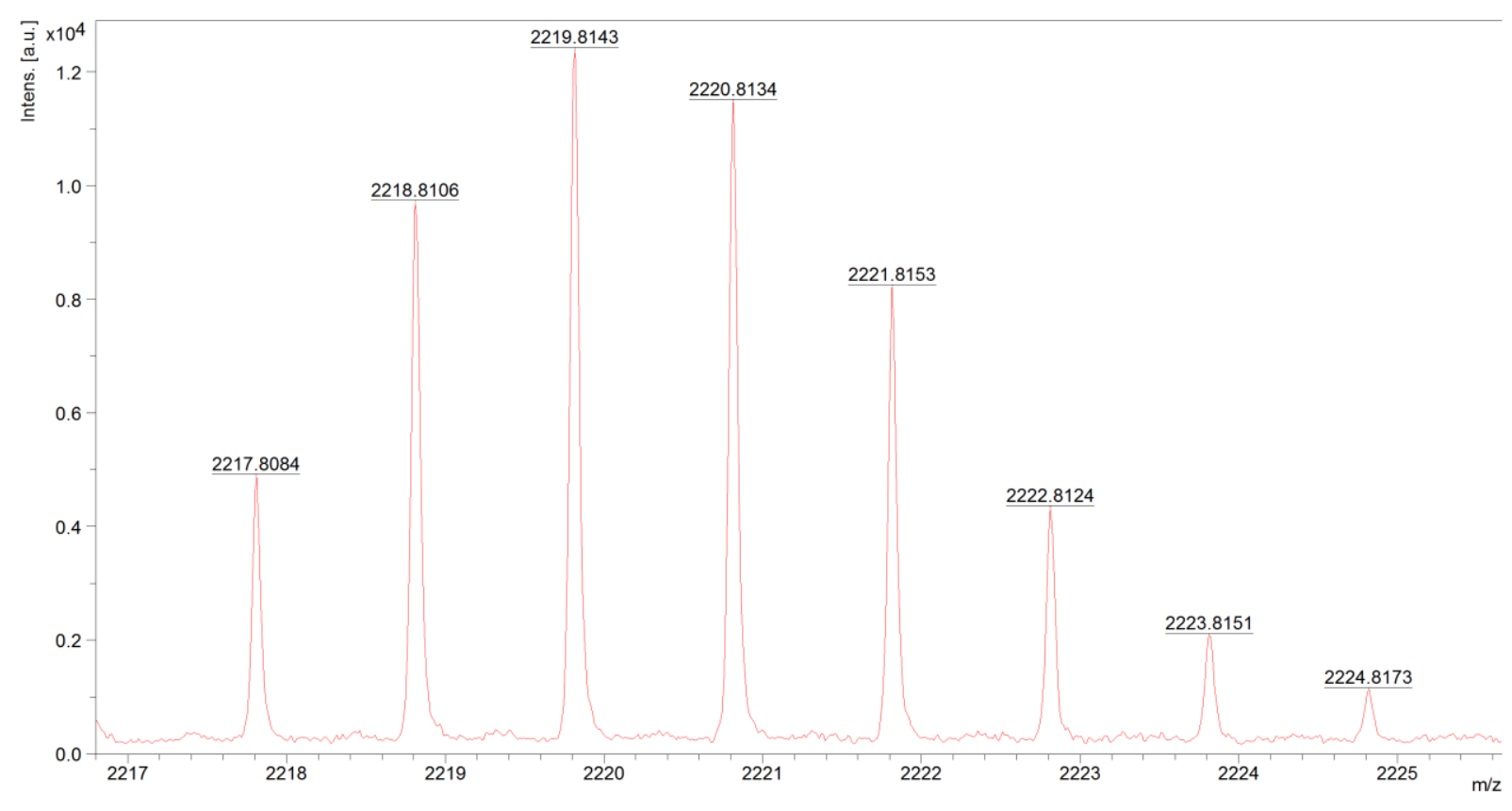


Compound $1 \mathrm{c}$

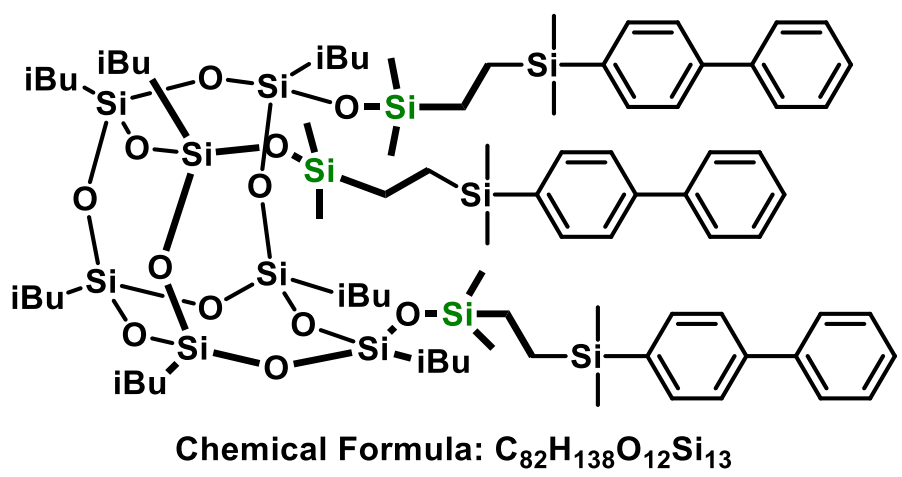

${ }^{1} \mathrm{H}$ NMR (300 MHz, Chloroform-d) $\delta 7.68-7.56(\mathrm{~m}, 15 \mathrm{H}), 7.52-7.42(\mathrm{~m}, 8 \mathrm{H}), 7.42-7.29(\mathrm{~m}, 4 \mathrm{H}), 1.98$ $-1.75(\mathrm{~m}, 7 \mathrm{H}), 0.98(\mathrm{dd}, J=6.6,2.8 \mathrm{~Hz}, 42 \mathrm{H}), 0.79-0.48(\mathrm{~m}, 26 \mathrm{H}), 0.30(\mathrm{~s}, 16 \mathrm{H}), 0.15(\mathrm{~s}, 20 \mathrm{H})$.

${ }^{13} \mathrm{C}$ NMR $\left(101 \mathrm{MHz}, \mathrm{CDCl}_{3}\right) \delta 141.65,141.36,138.50,134.85,128.93,128.90,128.88,127.42,127.40$, $127.32,127.29,126.76,126.58,26.21,26.03,25.79,25.24,24.26,24.15,24.02,23.94,22.62,10.26$, $7.22,-0.18,-3.35$.

${ }^{29} \mathrm{Si} \mathrm{NMR}\left(79 \mathrm{MHz}, \mathrm{CDCl}_{3}\right)$ $\delta 9.69,-1.31,-67.28,-67.76,-68.12$.

MALDI-TOF MS (m/z): calcd. for $\mathrm{C}_{82} \mathrm{H}_{138} \mathrm{NaO}_{12} \mathrm{Si}_{13}$ 1704,09; found 1703,74.

\section{${ }^{1} \mathrm{H}$ NMR $\left(300 \mathrm{MHz}, \mathrm{CDCl}_{3}\right)$}
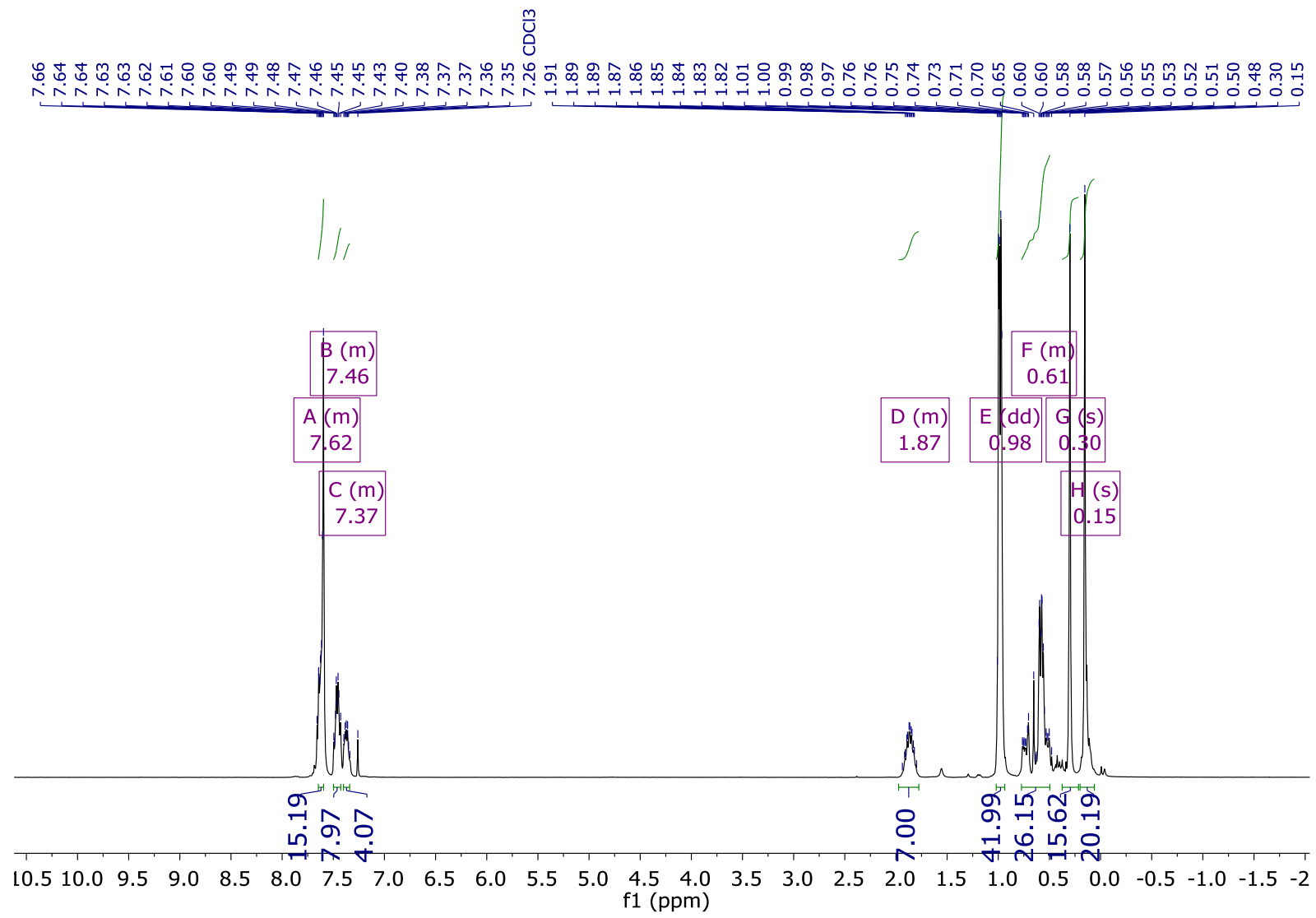
${ }^{13} \mathrm{C}$ NMR (101 MHz, $\left.\mathrm{CDCl}_{3}\right)$

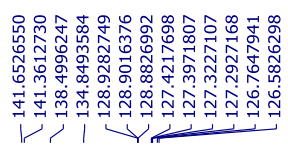

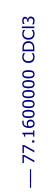
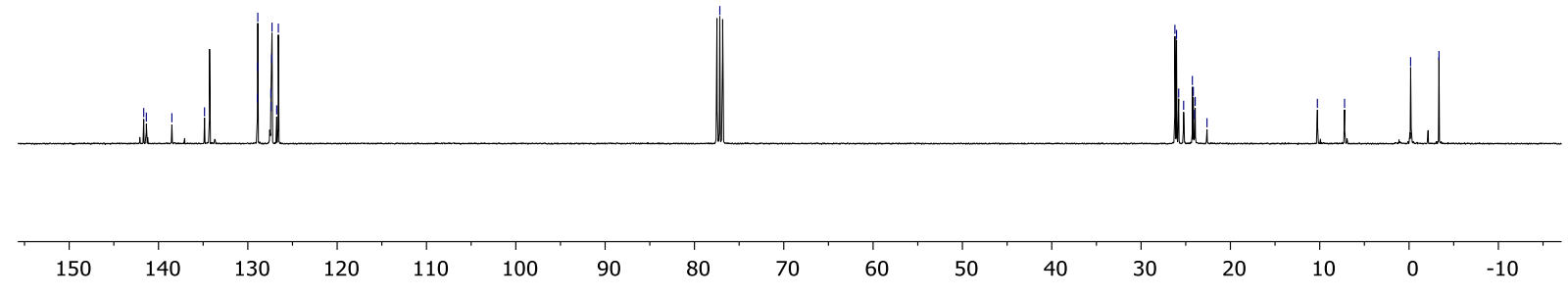

${ }^{29} \mathrm{Si}$ NMR $\left(79 \mathrm{MHz}, \mathrm{CDCl}_{3}\right)$
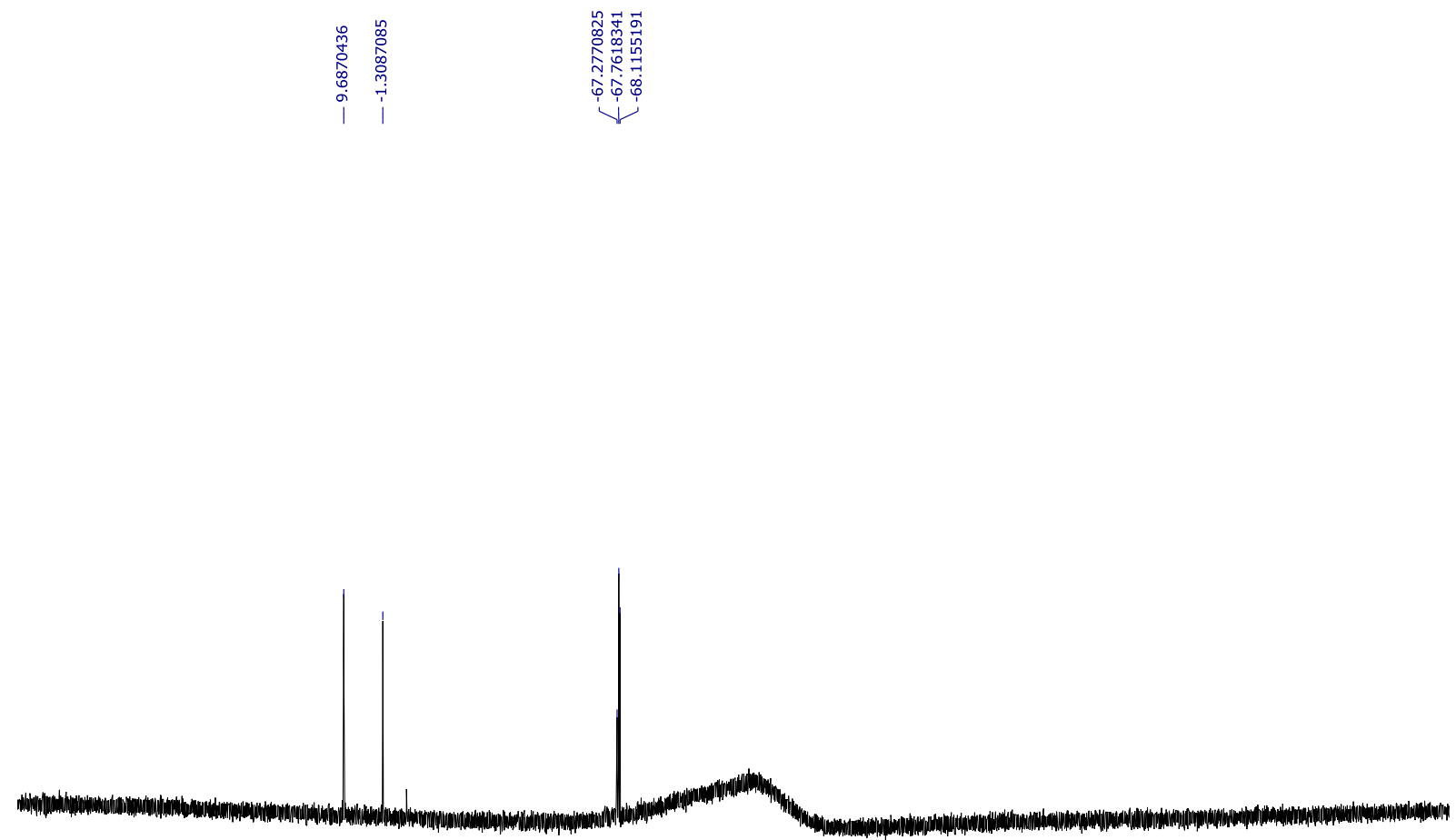

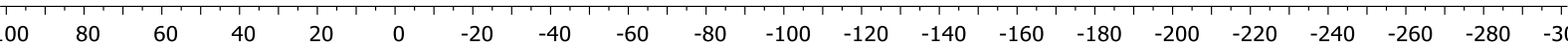


MALDI-TOF MS

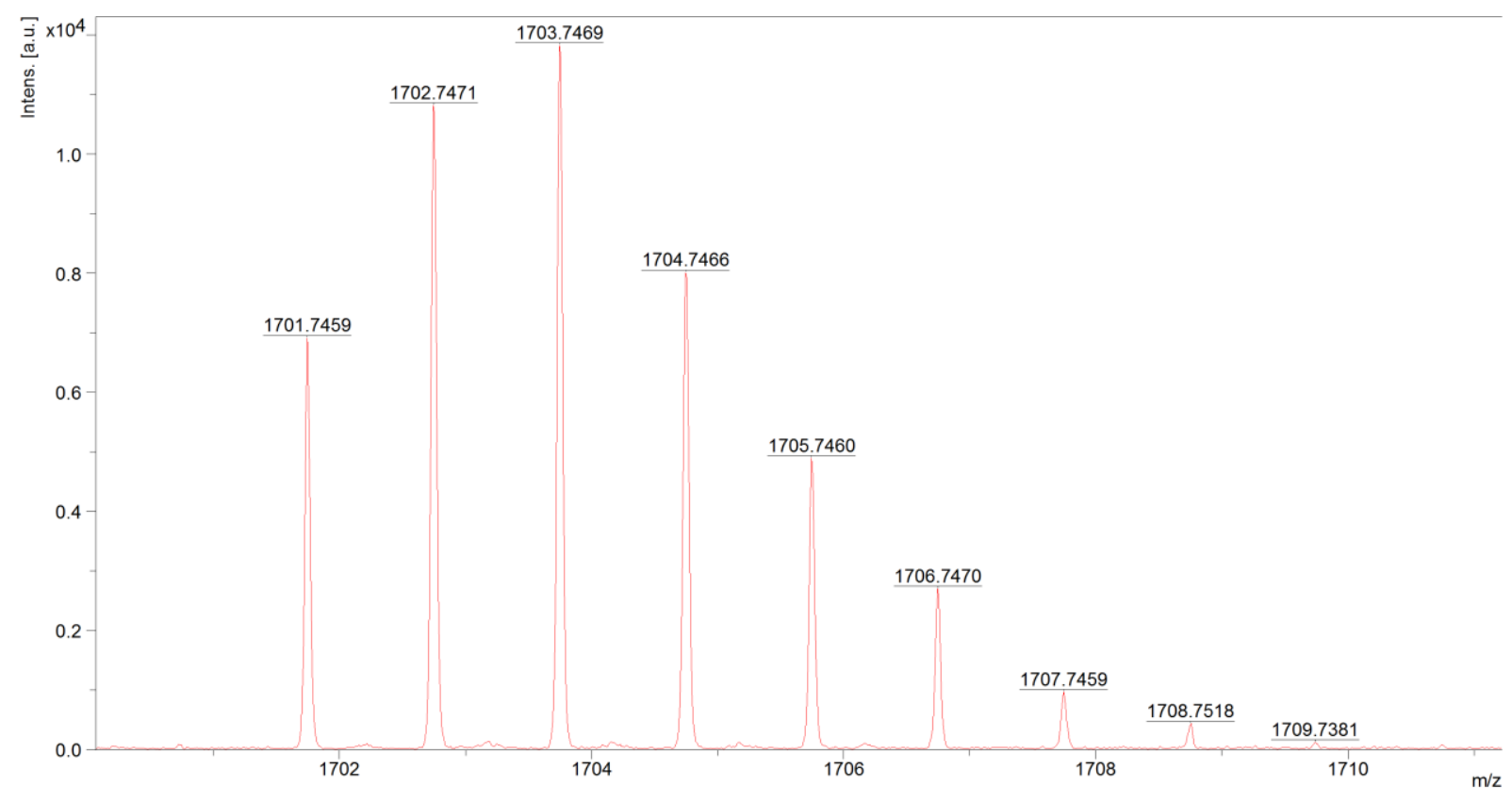


Compound 2c

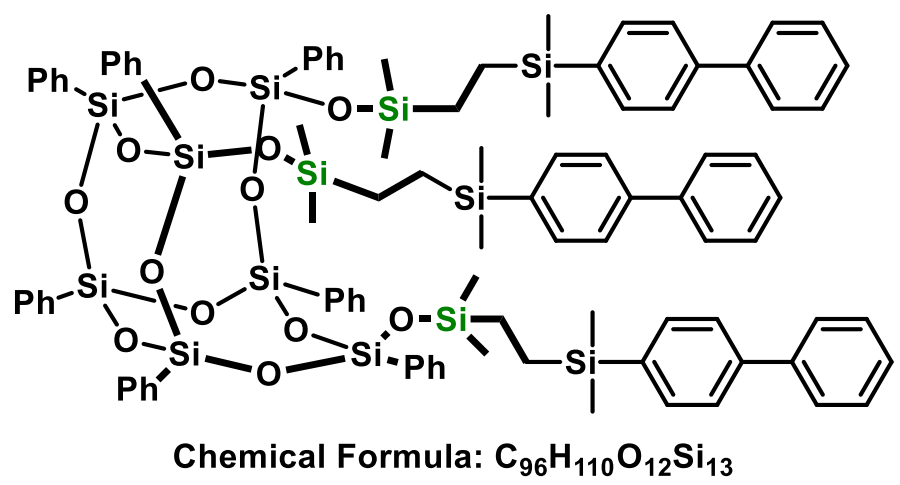

${ }^{1} \mathrm{H}$ NMR (300 MHz, Chloroform-d) $\delta 7.68-7.55(\mathrm{~m}, 15 \mathrm{H}), 7.54-7.40(\mathrm{~m}, 26 \mathrm{H}), 7.39-7.27(\mathrm{~m}, 20 \mathrm{H})$, $0.73-0.63(\mathrm{~m}, 6 \mathrm{H}), 0.62-0.51(\mathrm{~m}, 6 \mathrm{H}), 0.28(\mathrm{~s}, 16 \mathrm{H}), 0.21(\mathrm{~s}, 16 \mathrm{H}), 0.10(\mathrm{~d}, J=4.2 \mathrm{~Hz}, 4 \mathrm{H})$.

${ }^{13} \mathrm{C}$ NMR $\left(101 \mathrm{MHz}, \mathrm{CDCl}_{3}\right) \delta 141.57,141.34,138.37,134.84,134.26,134.16,134.12,133.11,131.33$, $130.23,130.07,129.19,128.92,128.90,128.87,128.38,127.98,127.70,127.64,127.41,127.32$, $127.28,126.76,126.53,125.45,10.23,7.13,-0.13,-3.44$.

${ }^{29} \mathrm{Si} \mathrm{NMR}\left(79 \mathrm{MHz}, \mathrm{CDCl}_{3}\right) \delta 12.36,-1.35,-77.43,-77.91,-78.20$.

MALDI-TOF MS (m/z): calcd. for $\mathrm{C}_{96} \mathrm{H}_{110} \mathrm{O}_{12} \mathrm{Si}_{13} \mathrm{Na}$ 1844,02; found 1843,49.

\section{${ }^{1} \mathrm{H}$ NMR $\left(300 \mathrm{MHz}, \mathrm{CDCl}_{3}\right)$}

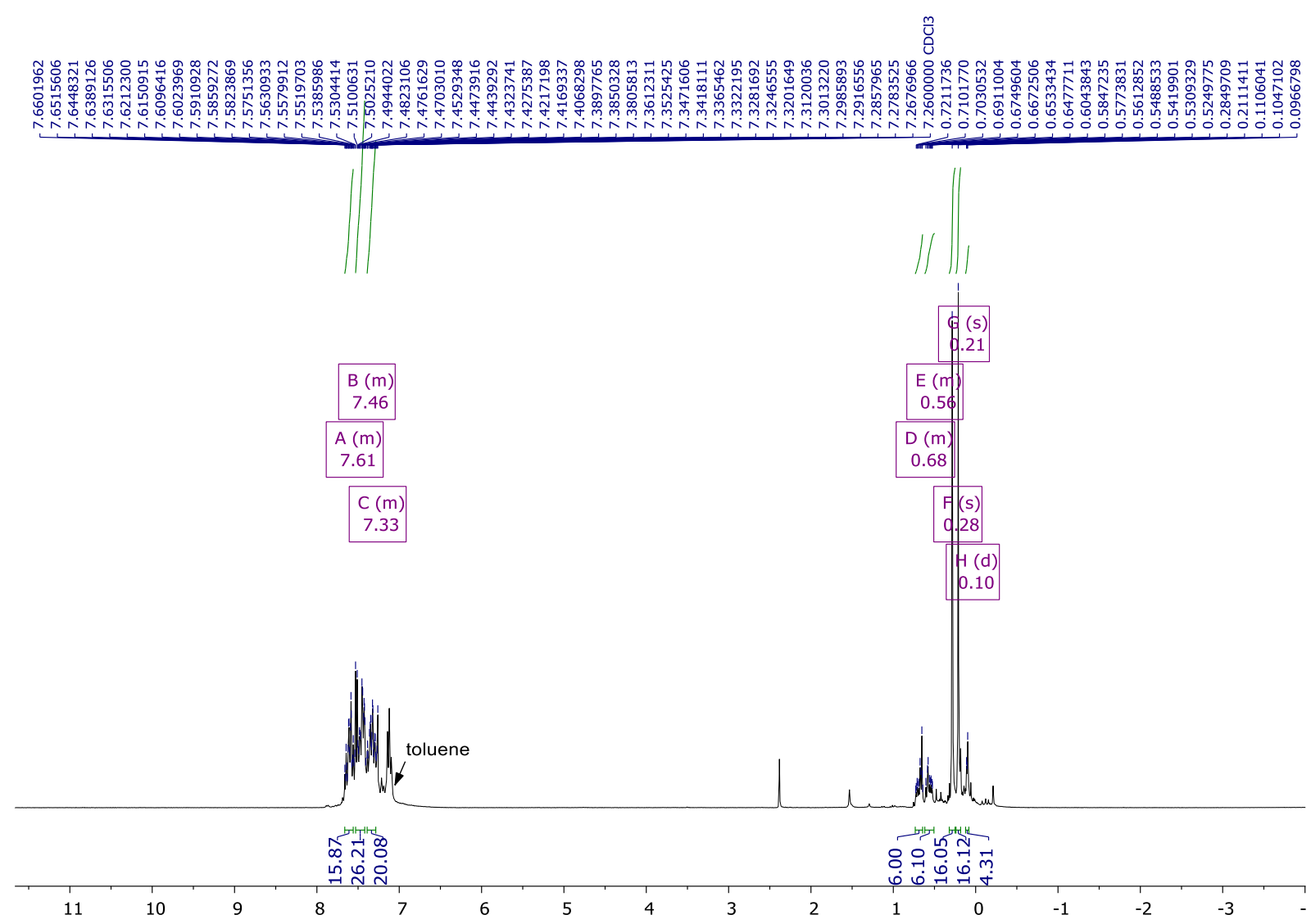


${ }^{13} \mathrm{C}$ NMR (101 MHz, $\mathrm{CDCl}_{3}$
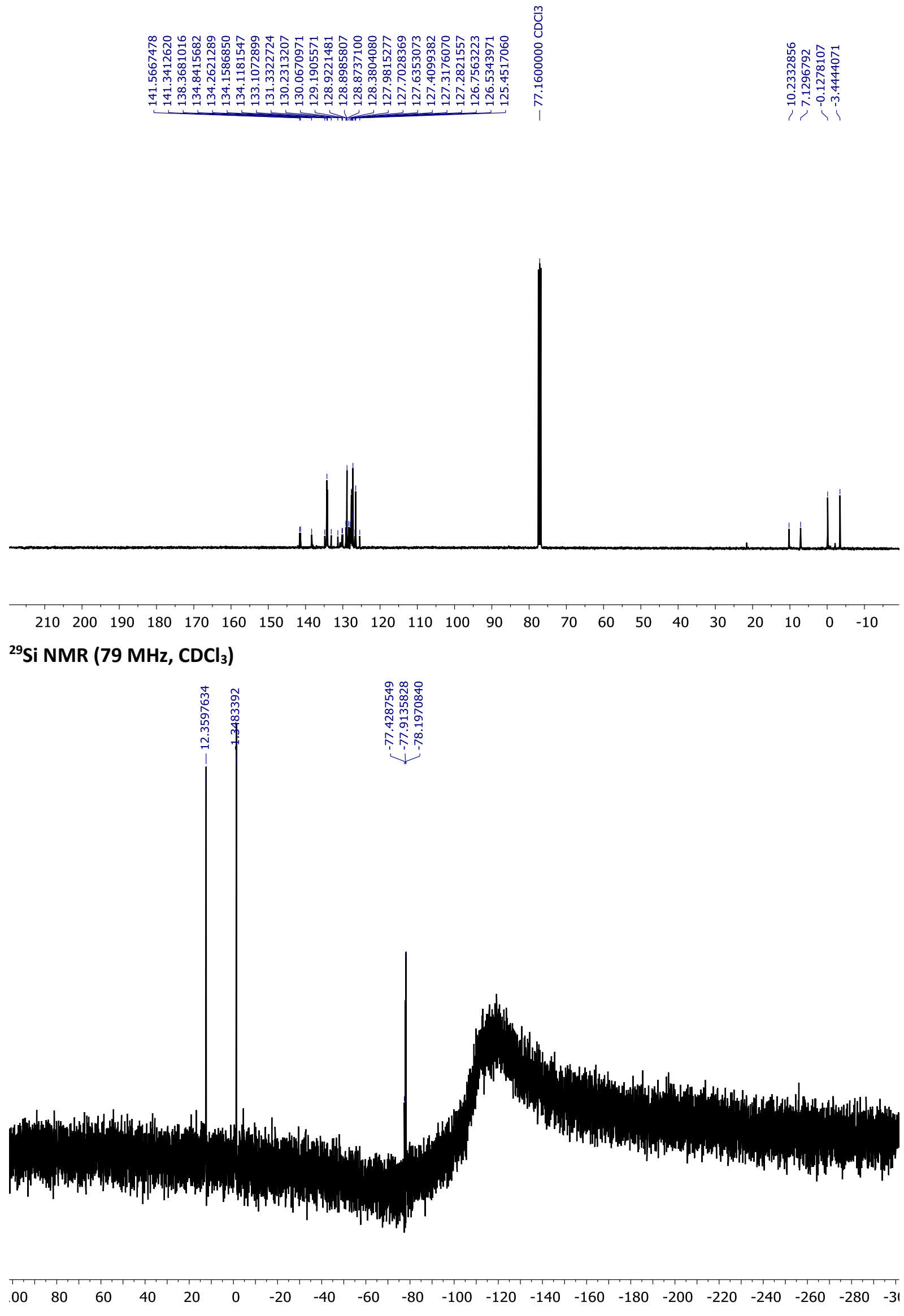
MALDI-TOF MS

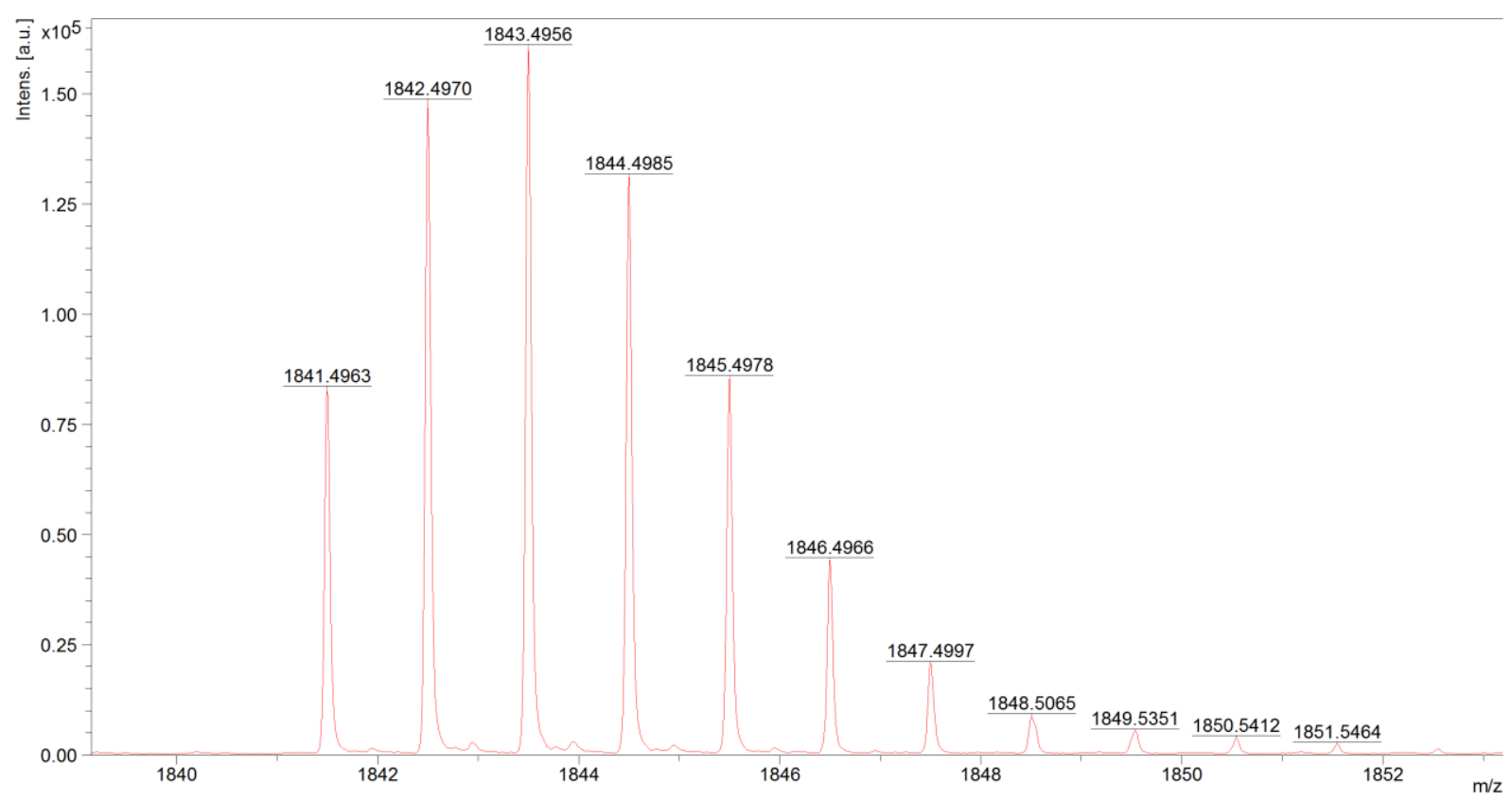


Compound $3 c$

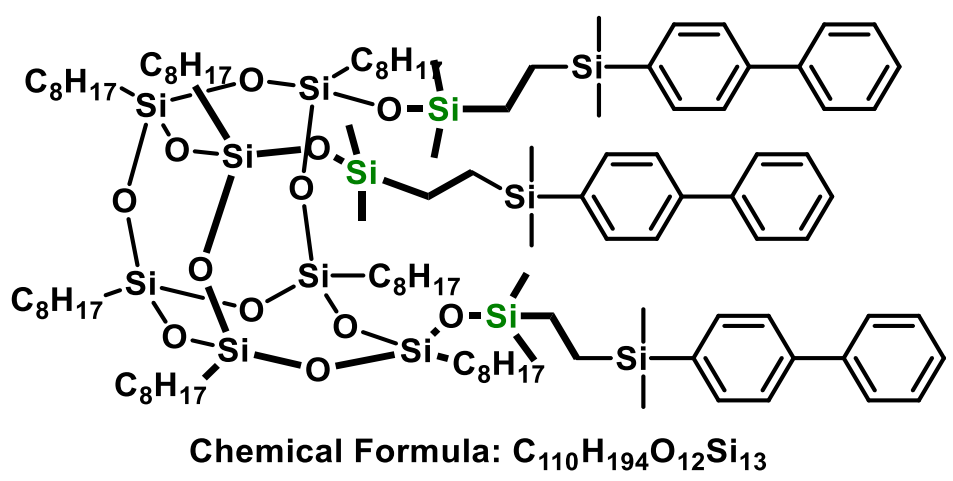

${ }^{1} \mathrm{H}$ NMR (300 MHz, Chloroform-d) $\delta 7.68-7.55(\mathrm{~m}, 18 \mathrm{H}), 7.52-7.30(\mathrm{~m}, 9 \mathrm{H}), 1.84(\mathrm{~s}, 7 \mathrm{H}), 1.35-1.20$ (m, 7H), $1.23-1.09(\mathrm{~m}, 7 \mathrm{H}), 1.02(\mathrm{~d}, J=6.6 \mathrm{~Hz}, 20 \mathrm{H}), 0.91(\mathrm{~d}, J=3.0 \mathrm{~Hz}, 68 \mathrm{H}), 0.82-0.65(\mathrm{~m}, 10 \mathrm{H})$, $0.64(\mathrm{~s}, 2 \mathrm{H}), 0.61-0.47(\mathrm{~m}, 10 \mathrm{H}), 0.29(\mathrm{~d}, \mathrm{~J}=2.0 \mathrm{~Hz}, 15 \mathrm{H}), 0.22(\mathrm{~s}, 5 \mathrm{H}), 0.15(\mathrm{~s}, 16 \mathrm{H})$.

${ }^{13} \mathrm{C}$ NMR $\left(101 \mathrm{MHz}, \mathrm{CDCl}_{3}\right) \delta$ 141.64, 141.37, 139.30, 138.49, 134.85, 134.28, 131.96, 128.93, 128.91, $128.88,127.56,127.41,127.33,127.30,126.77,126.58,55.23,54.75,54.60,31.33,30.45$, 30.37, 30.30, $29.61,26.57,25.87,25.48,25.37,25.26,25.16,25.05,10.29,7.29,0.65,-0.12,-2.13,-3.34$.

${ }^{29} \mathrm{Si} \mathrm{NMR}\left(79 \mathrm{MHz}, \mathrm{CDCl}_{3}\right) \delta 9.67,9.58,9.53,-1.30,-67.50,-68.02,-68.22$.

MALDI-TOF MS (m/z): calcd. for $\mathrm{C}_{110} \mathrm{H}_{194} \mathrm{NaO}_{12} \mathrm{Si}_{13}$ 2096,84; found 1096,15.

\section{${ }^{1} \mathrm{H}$ NMR $\left(300 \mathrm{MHz}, \mathrm{CDCl}_{3}\right)$}

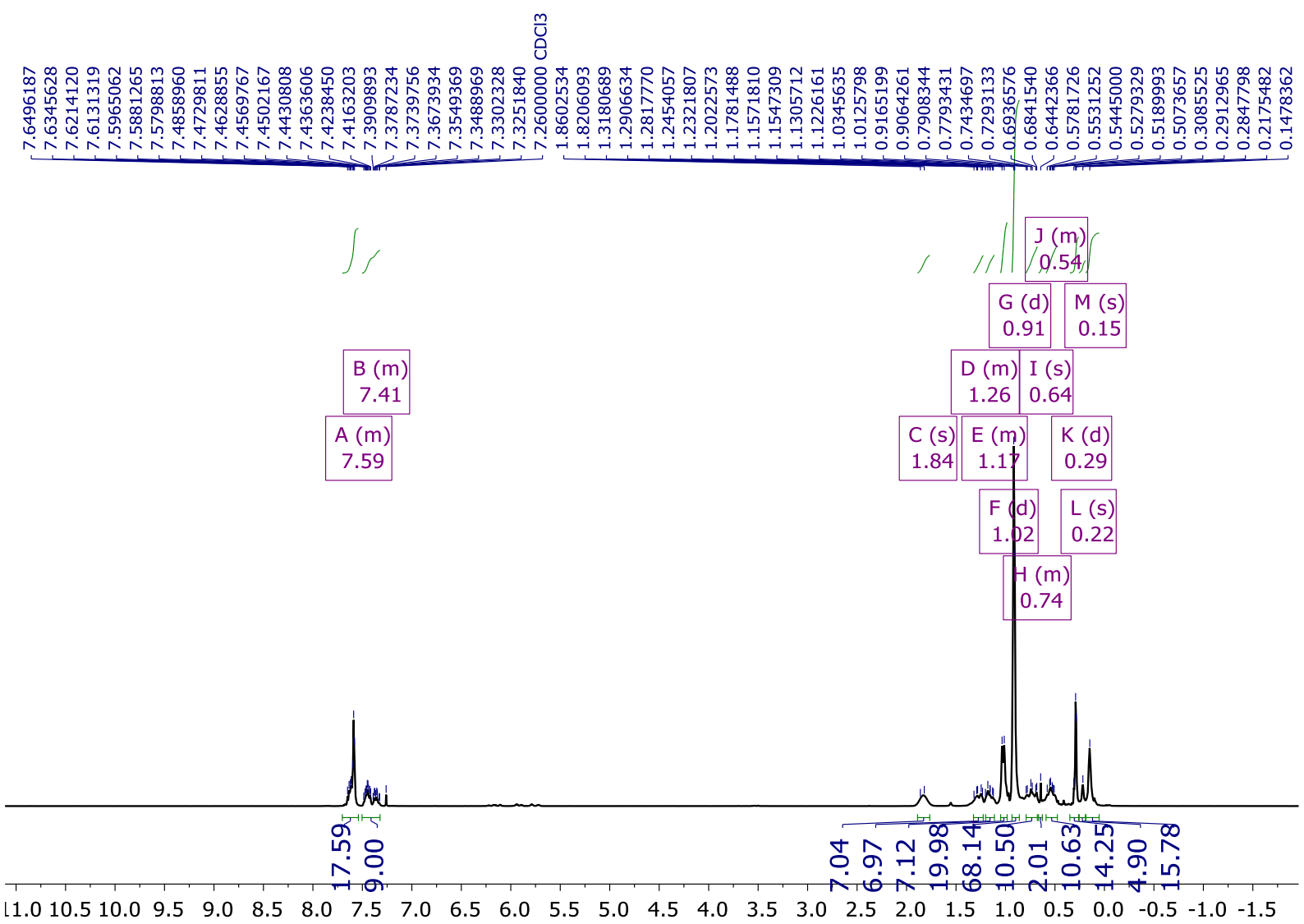


${ }^{13} \mathrm{C}$ NMR (101 MHz, $\mathrm{CDCl}_{3}$ )
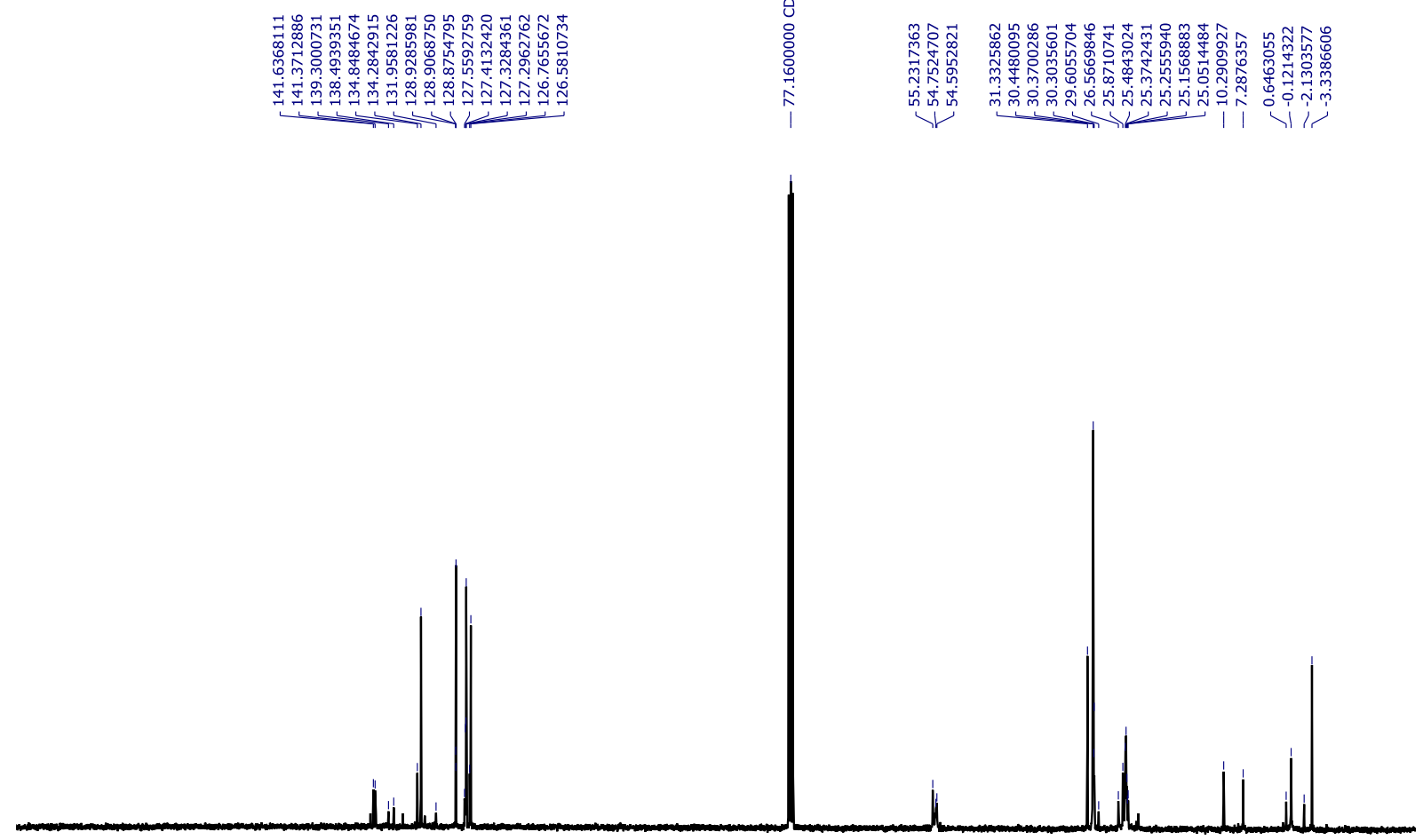

$\begin{array}{lllllllllllllllllllll}190 & 180 & 170 & 160 & 150 & 140 & 130 & 120 & 110 & 100 & 90 & 80 & 70 & 60 & 50 & 40 & 30 & 20 & 10 & 0 & -10\end{array}$ ${ }^{29} \mathrm{Si} \mathrm{NMR} \mathrm{(79} \mathrm{MHz,} \mathrm{CDCl}_{3}$ )
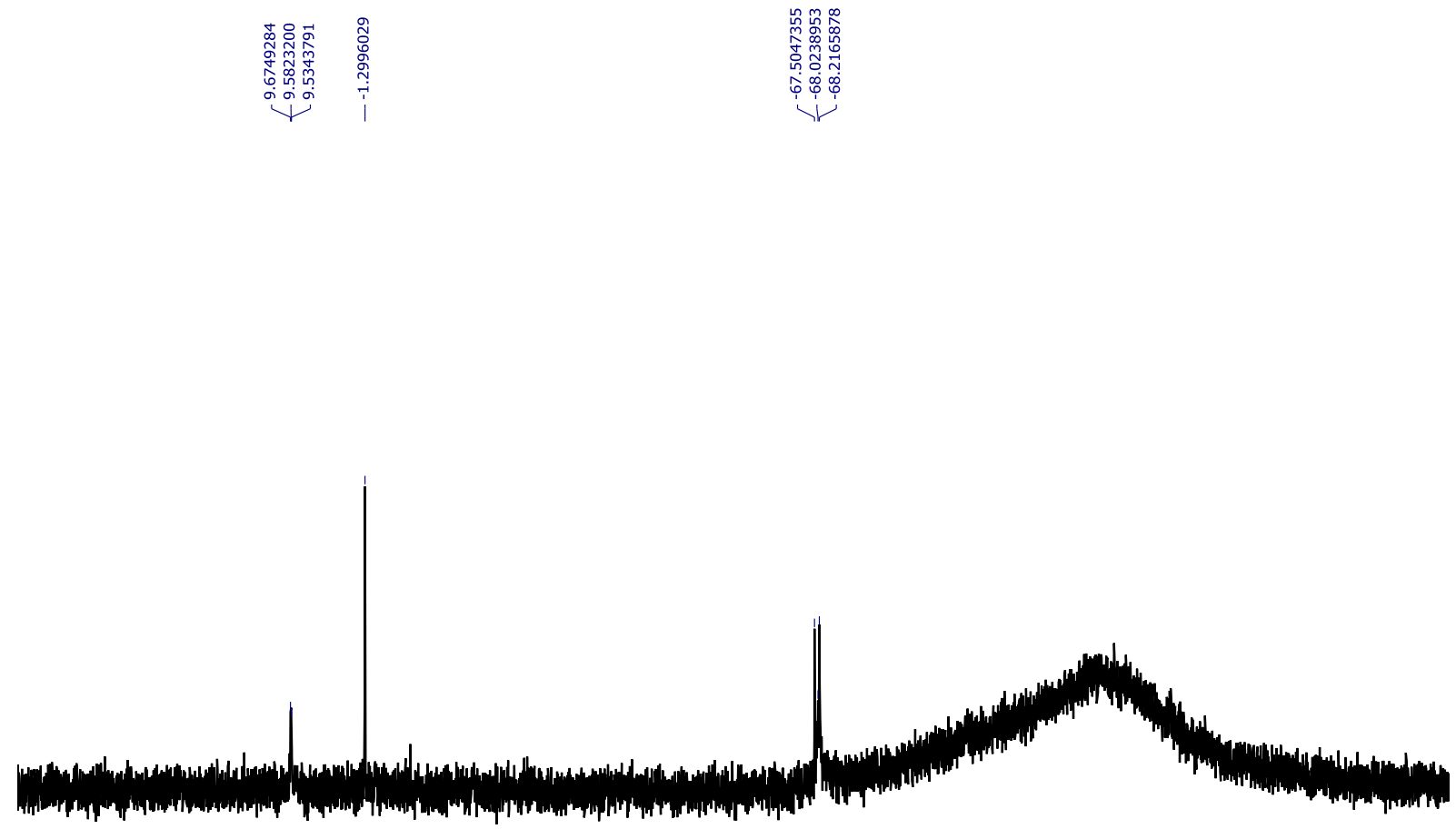

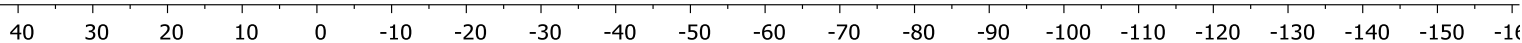


MALDI-TOF MS

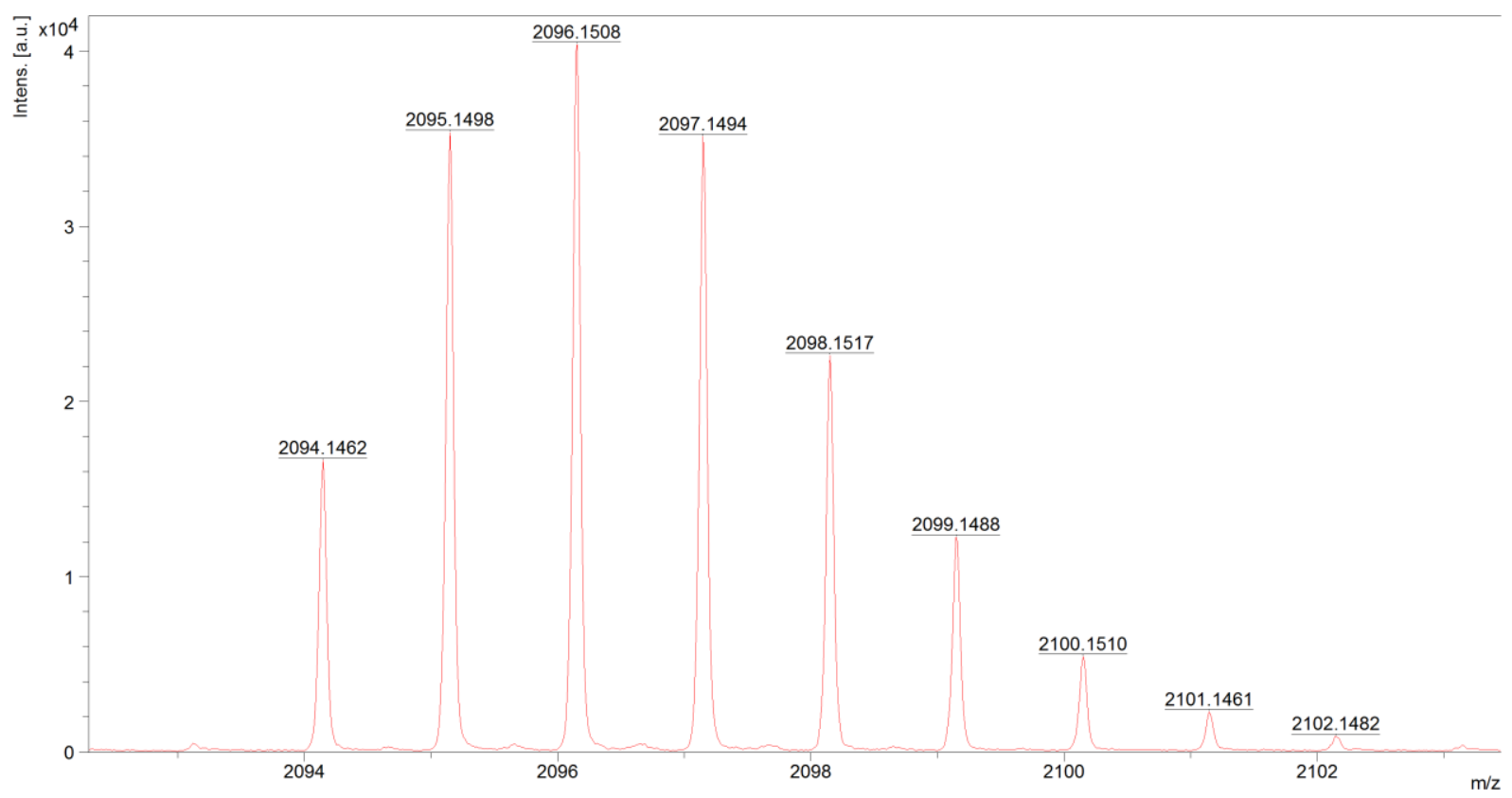


Compound $4 \mathrm{c}$

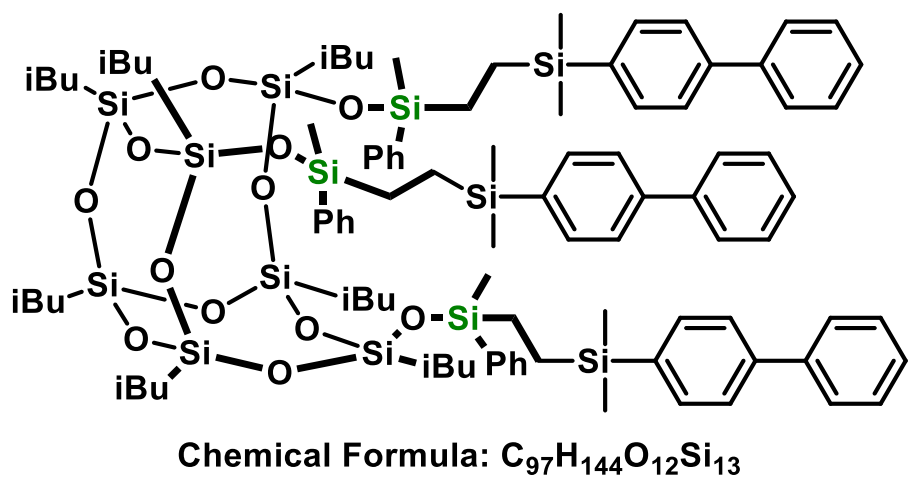

${ }^{1} \mathrm{H}$ NMR (300 MHz, Chloroform-d) $87.67-7.41(\mathrm{~m}, 34 \mathrm{H}), 7.41-7.34(\mathrm{~m}, 4 \mathrm{H}), 7.31-7.26(\mathrm{~m}, 2 \mathrm{H}), 7.25$

$-7.20(\mathrm{~m}, 2 \mathrm{H}), 1.98-1.72(\mathrm{~m}, 7 \mathrm{H}), 1.01-0.86(\mathrm{~m}, 42 \mathrm{H}), 0.76-0.47(\mathrm{~m}, 26 \mathrm{H}), 0.43-0.09(\mathrm{~m}, 27 \mathrm{H})$.

${ }^{13} \mathrm{C}$ NMR $\left(101 \mathrm{MHz}, \mathrm{CDCl}_{3}\right) \delta 142.08,141.64,141.39,141.36,141.20,138.43,138.38,138.29,138.25$, $137.07,134.84,134.28,133.63,129.18,128.92,128.89,128.87,127.69,127.65,127.61,127.55$, $127.41,127.39,127.31,127.29,126.76,126.60,126.55,77.16,26.23,26.18,26.07,26.04,26.01$, 25.79, $25.22,24.19,24.12,24.00,23.92,22.60,9.10,8.05,7.06,-1.39,-1.50,-1.58,-2.13,-3.36$.

${ }^{29} \mathrm{Si} \mathrm{NMR}\left(79 \mathrm{MHz}, \mathrm{CDCl}_{3}\right) \delta-0.45,-0.50,-0.56,-1.27,-67.22,-67.36,-67.59$.

MALDI-TOF MS (m/z): calcd. for $\mathrm{C}_{97} \mathrm{H}_{144} \mathrm{O}_{12} \mathrm{Si}_{13} \mathrm{Na}$ 1890,30; found 1889,75.

\section{${ }^{1} \mathrm{H}$ NMR $\left(300 \mathrm{MHz}, \mathrm{CDCl}_{3}\right)$}

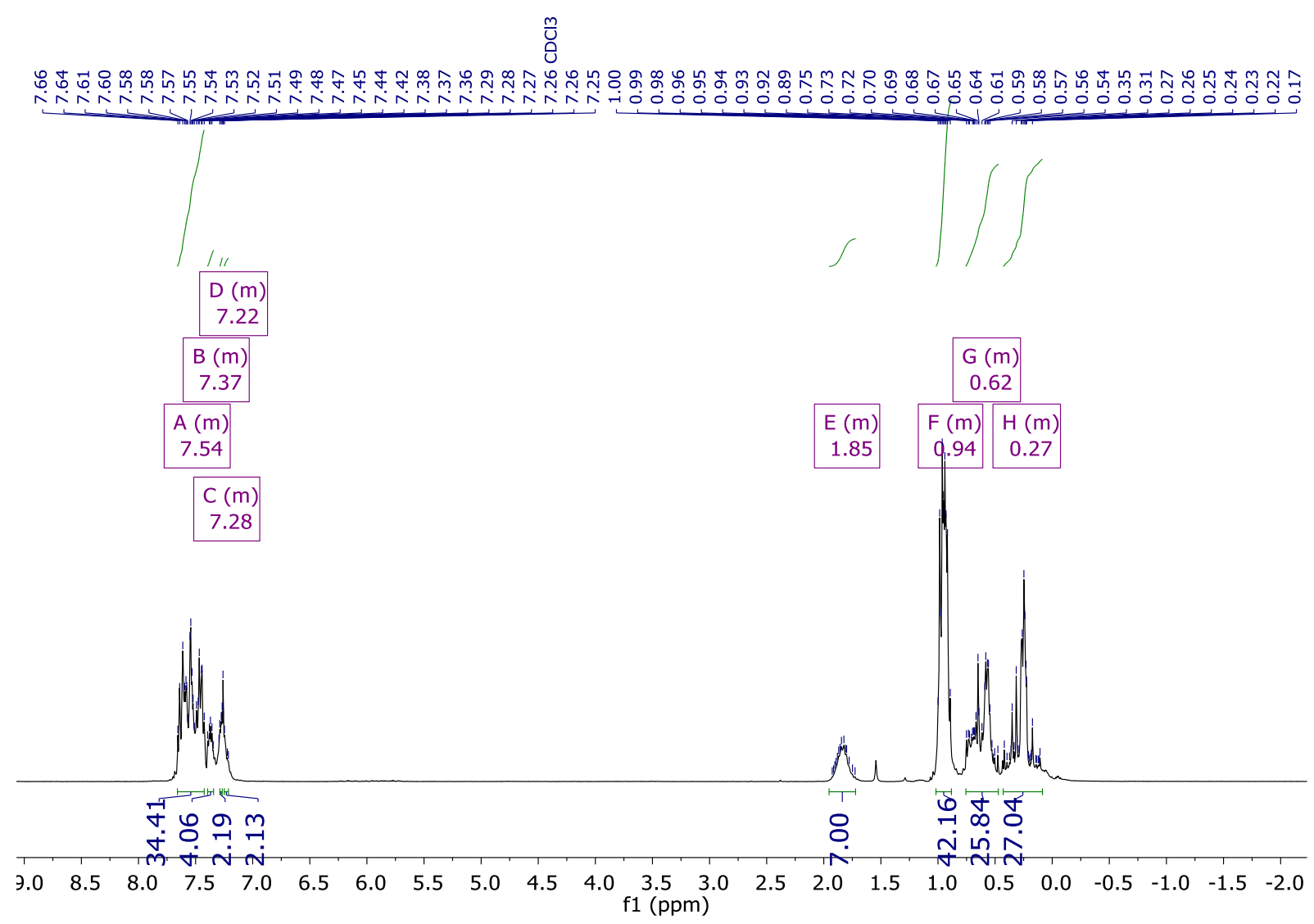




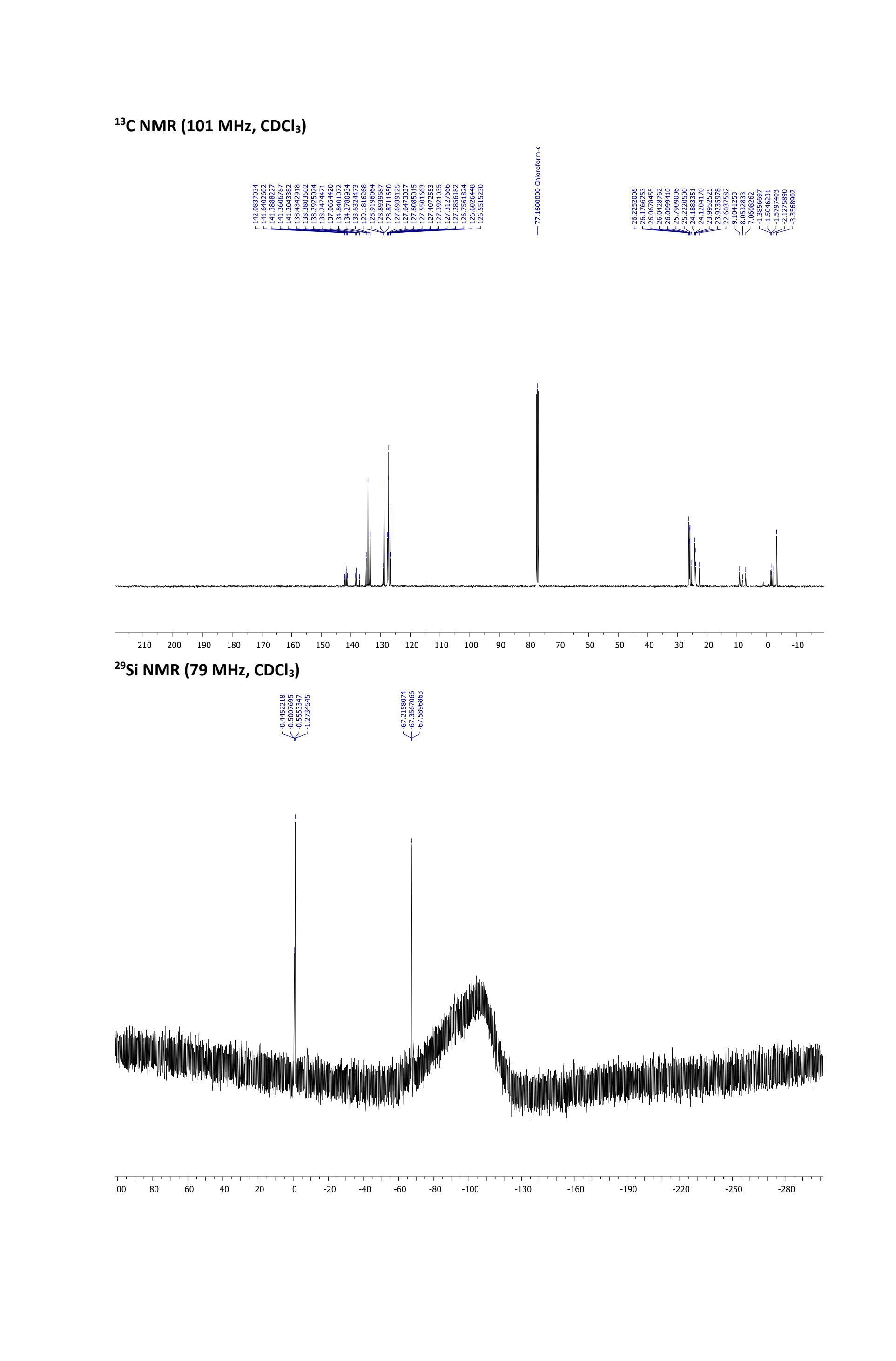


MALDI-TOF MS

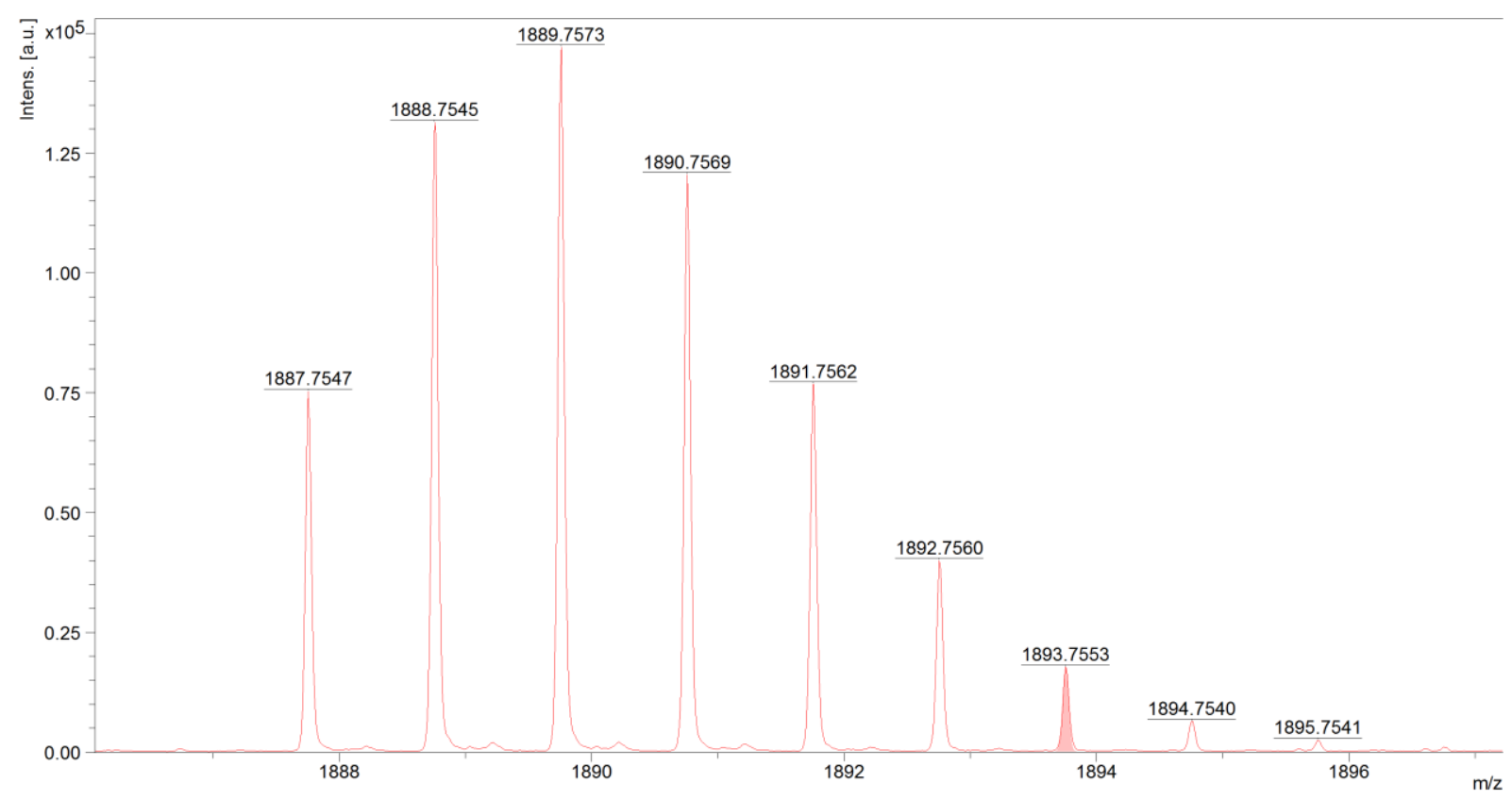


Compound 7c

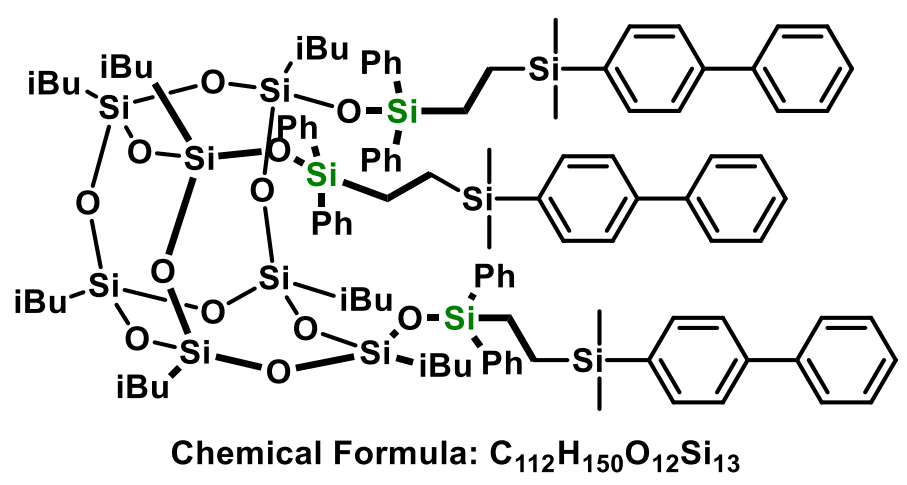

${ }^{1} \mathrm{H}$ NMR (300 MHz, Chloroform-d) $\delta 7.73-7.27(\mathrm{~m}, 38 \mathrm{H}), 7.28-6.94(\mathrm{~m}, 19 \mathrm{H}), 1.97-1.67(\mathrm{~m}, 7 \mathrm{H})$, $1.04-0.81(\mathrm{~m}, 22 \mathrm{H}), 0.74-0.34(\mathrm{~m}, 26 \mathrm{H}), 0.33--0.07(\mathrm{~m}, 18 \mathrm{H})$

${ }^{13} \mathrm{C}$ NMR $\left(75 \mathrm{MHz}, \mathrm{CDCl}_{3}\right) \delta 139.39,136.07,134.53,133.79,133.16,129.43,128.85,127.75,26.10$, $26.05,25.80,25.46,24.00,22.65,7.02,0.99,-3.50$.

${ }^{29} \mathrm{Si}$ NMR $\left(79 \mathrm{MHz}, \mathrm{CDCl}_{3}\right) \delta-1.20,-1.21,-1.26,-11.59,-11.64,-11.77,-66.01,-66.24,-66.76,-67.25,-$ $67.55,-67.59$.

MALDI-TOF MS (m/z): calcd. for $\mathrm{C}_{112} \mathrm{H}_{150} \mathrm{O}_{12} \mathrm{Si}_{13} \mathrm{Na} 2076,51$; found 2075,80.

\section{${ }^{1} \mathrm{H}$ NMR $\left(300 \mathrm{MHz}, \mathrm{CDCl}_{3}\right)$}
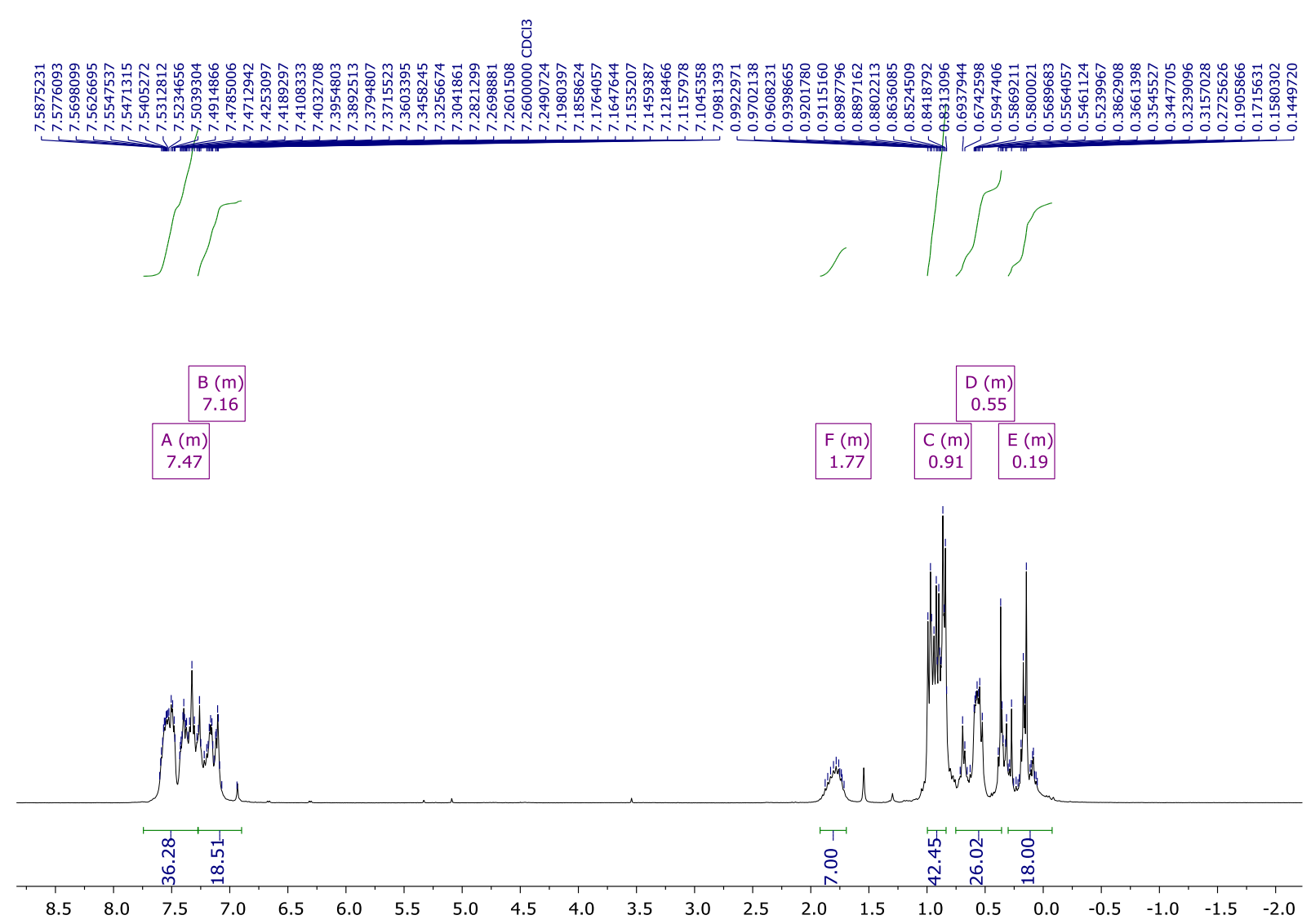
${ }^{13} \mathrm{C}$ NMR (101 MHz, $\left.\mathrm{CDCl}_{3}\right)$

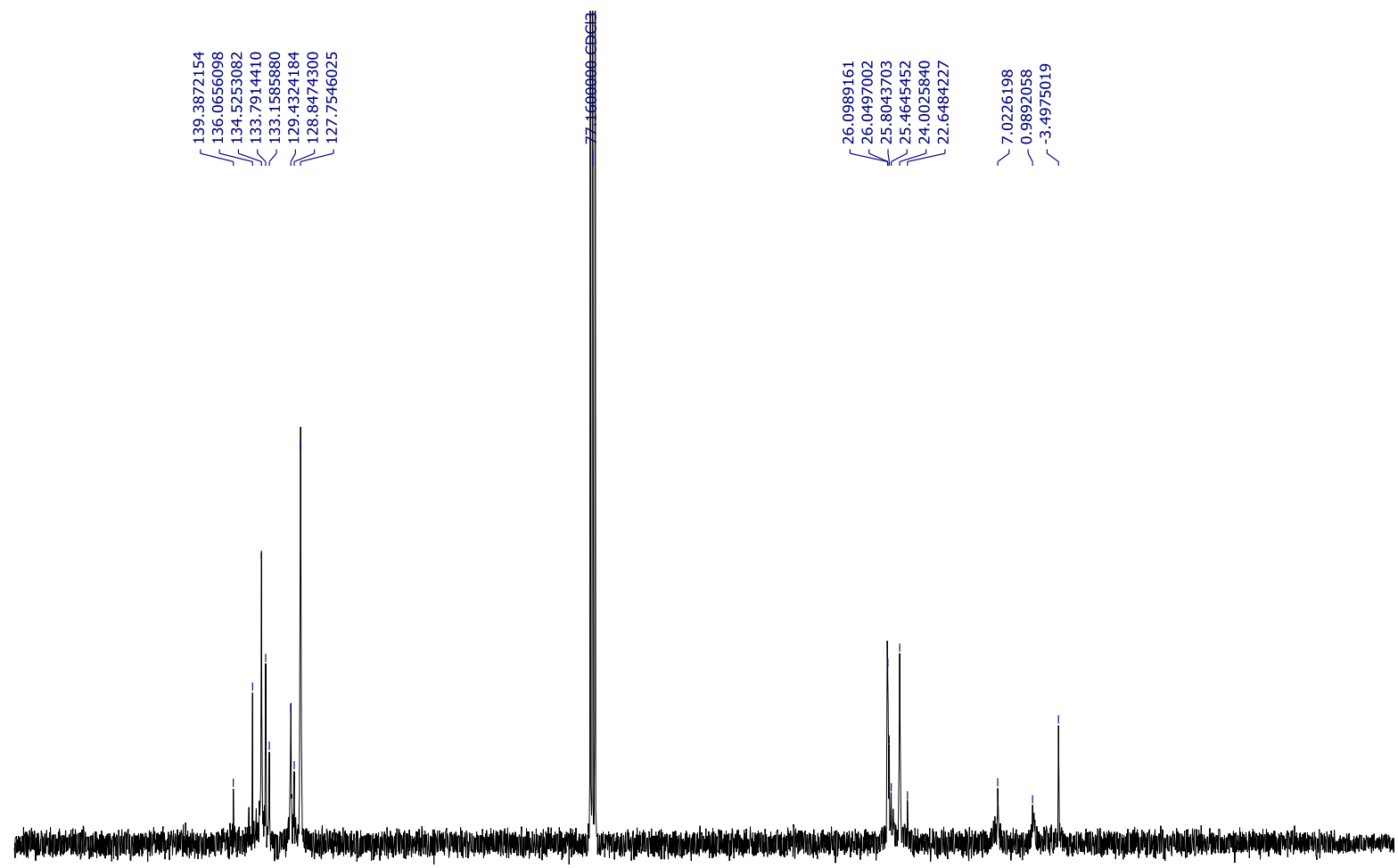

$\begin{array}{lllllllllllllllllllllllllllllllllll}170 & 160 & 150 & 140 & 130 & 120 & 110 & 100 & 90 & 80 & 70 & 60 & 50 & 40 & 30 & 20 & 10 & 0 & -10 & -20 & -30 & -40 & -50 & -60\end{array}$

${ }^{29}$ Si NMR (79 MHz, $\mathrm{CDCl}_{3}$ )
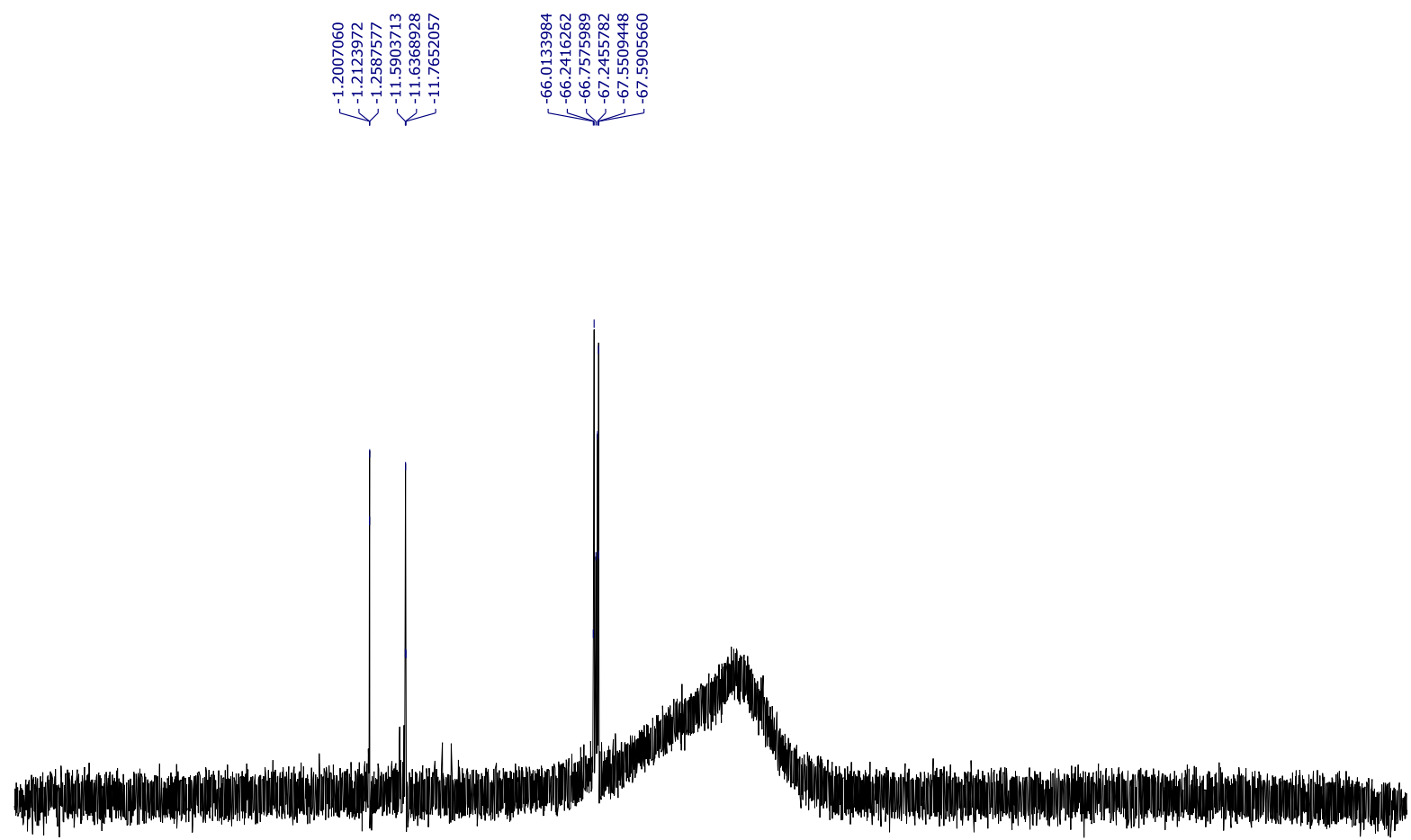

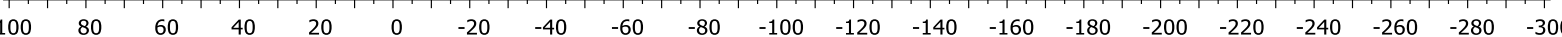


MALDI-TOF MS

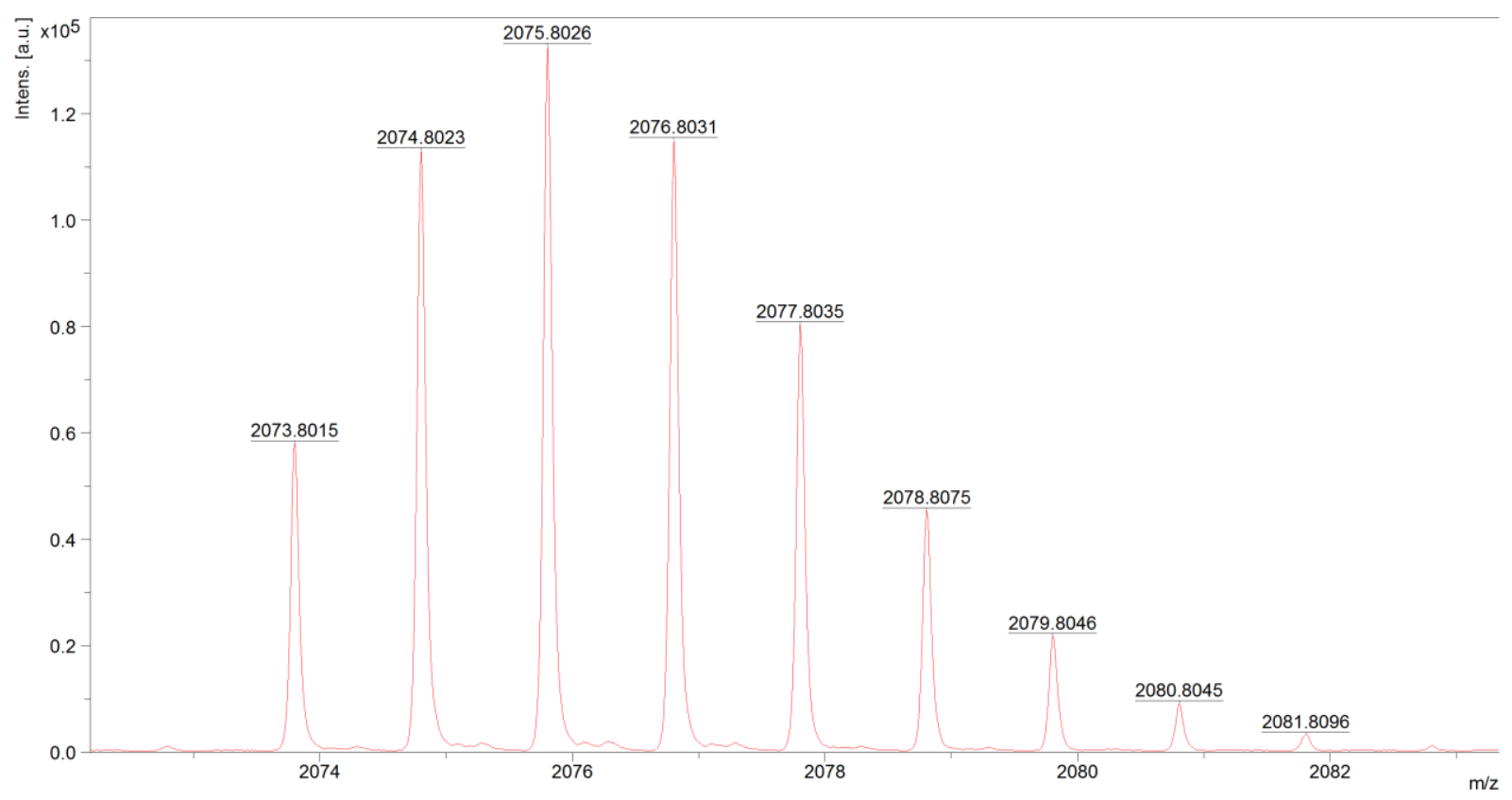


Compound $1 d$

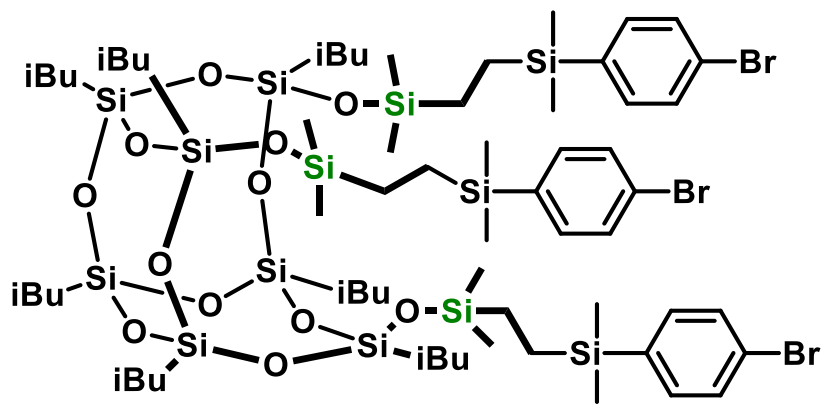

Chemical Formula: $\mathrm{C}_{64} \mathrm{H}_{123} \mathrm{Br}_{3} \mathrm{O}_{12} \mathrm{Si}_{13}$

${ }^{1} \mathrm{H}$ NMR $(400 \mathrm{MHz}$, ) $87.53-7.45(\mathrm{~m}, 6 \mathrm{H}), 7.41-7.32(\mathrm{~m}, 6 \mathrm{H}), 1.90-1.74(\mathrm{~m}, 7 \mathrm{H}), 1.01-0.91(\mathrm{~m}$, $42 \mathrm{H}), 0.70-0.60(\mathrm{~m}, 5 \mathrm{H}), 0.59-0.53(\mathrm{~m}, 16 \mathrm{H}), 0.50-0.38(\mathrm{~m}, 5 \mathrm{H}), 0.32(\mathrm{~s}, 3 \mathrm{H}), 0.24(\mathrm{~d}, J=1.6 \mathrm{~Hz}$, $14 \mathrm{H}), 0.19(\mathrm{~s}, 2 \mathrm{H}), 0.10(\mathrm{t}, 17 \mathrm{H})$.

${ }^{13} \mathrm{C}$ NMR $\left(101 \mathrm{MHz}, \mathrm{CDCl}_{3}\right) \delta$ 135.25, 130.84, 26.06, 25.91, 25.88, 25.85, 25.65, 25.07, 24.11, 24.06, $24.00,23.97,23.87,23.78,23.75,22.46,10.01,6.94,-0.37,-3.62$.

${ }^{29} \mathrm{Si} \mathrm{NMR}\left(79 \mathrm{MHz}, \mathrm{CDCl}_{3}\right)$ $\delta 9.57,9.52,9.49,-0.75,-0.75,-0.76,-67.27,-67.31,-67.73,-67.76,-67.79,-$ $67.98,-68.08,-68.11,-68.14$.

MALDI-TOF MS (m/z): calcd. for $\mathrm{C}_{64} \mathrm{H}_{123} \mathrm{Br}_{3} \mathrm{O}_{12} \mathrm{Si}_{13} \mathrm{Na}$ 1712,48; found 1711,35.

\section{${ }^{1} \mathrm{H}$ NMR $\left(300 \mathrm{MHz}, \mathrm{CDCl}_{3}\right)$}
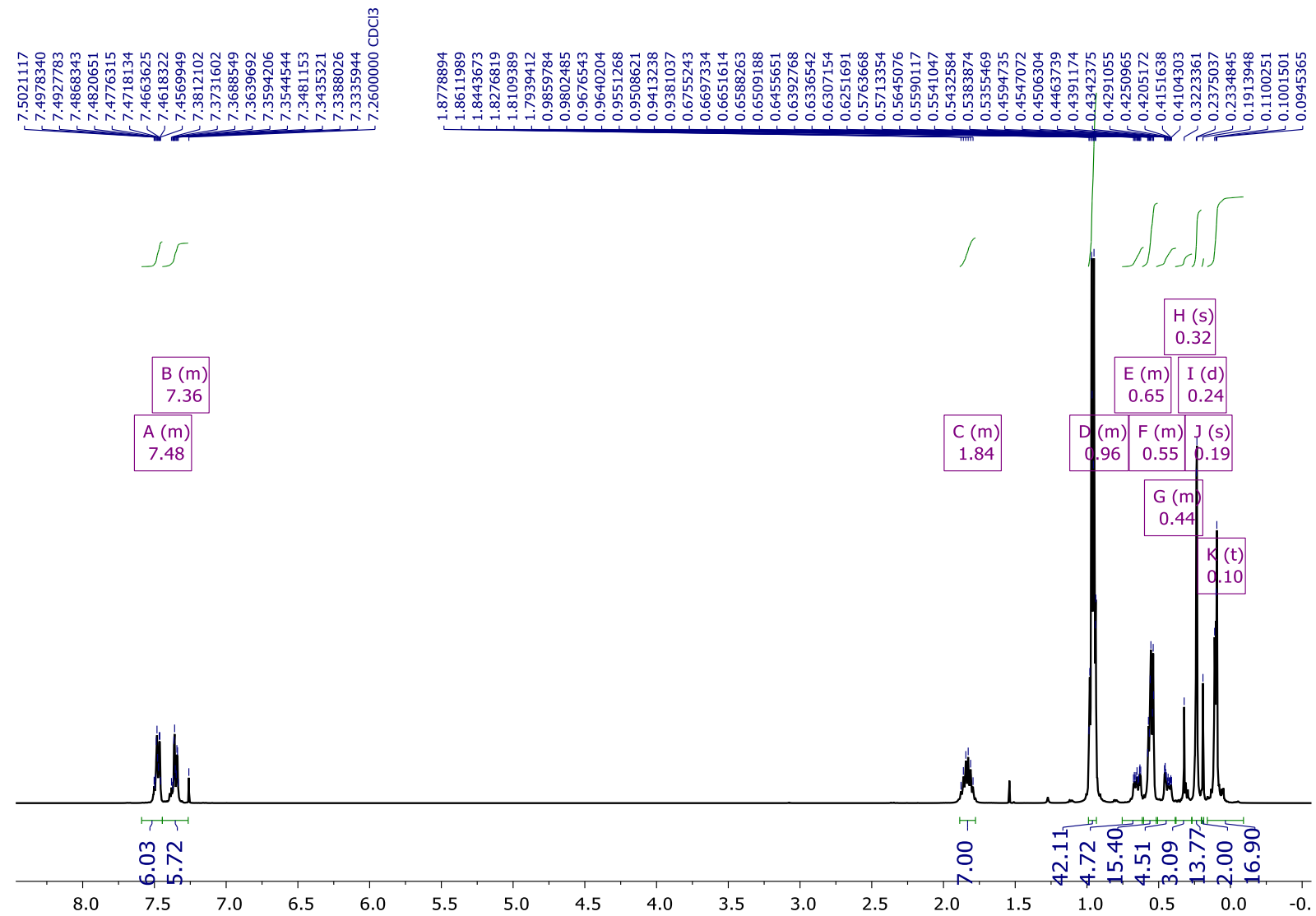
${ }^{13} \mathrm{C}$ NMR (101 MHz, $\mathrm{CDCl}_{3}$ )
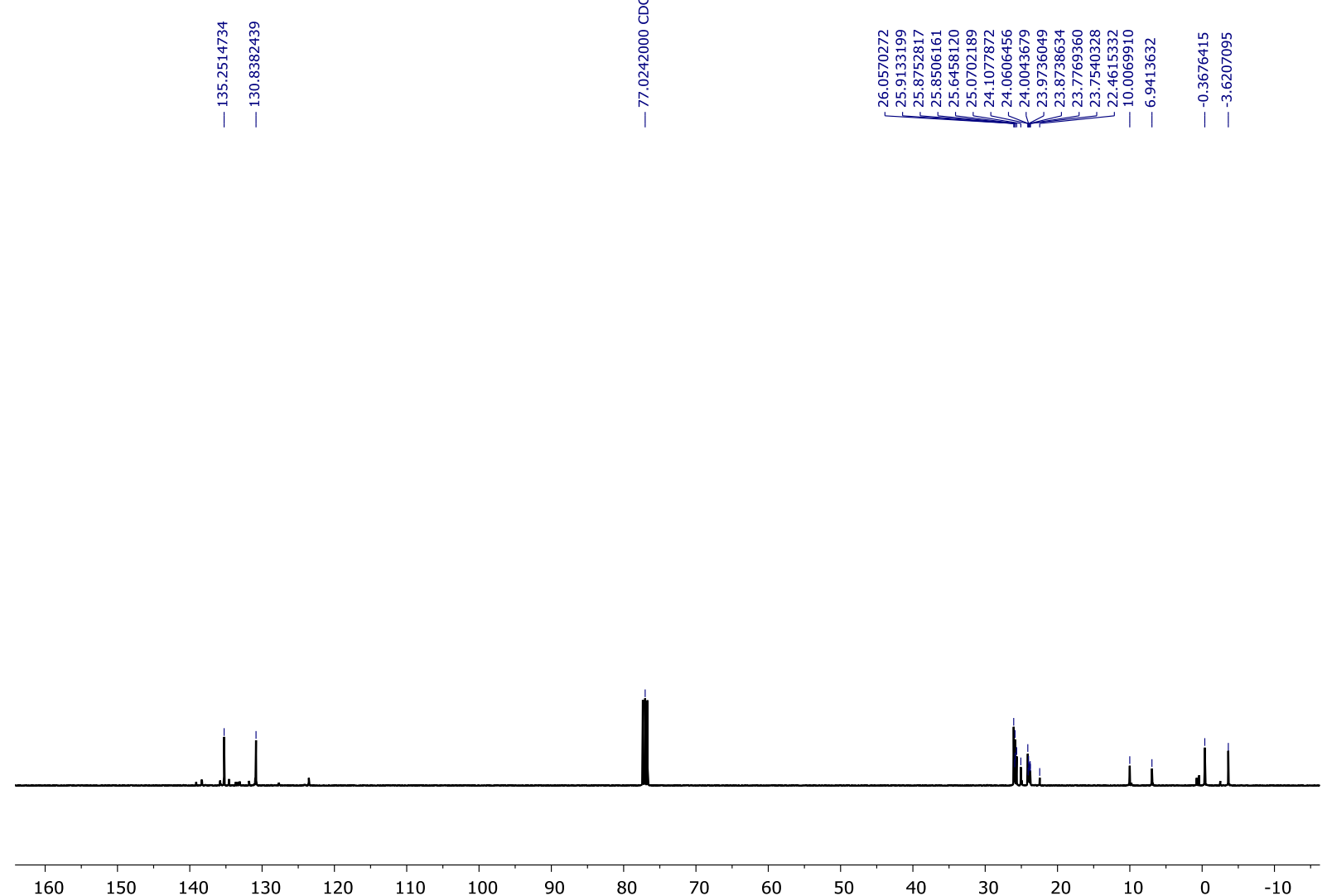

${ }^{29} \mathrm{Si} \mathrm{NMR} \mathrm{(79} \mathrm{MHz,} \mathrm{CDCl}_{3}$ )
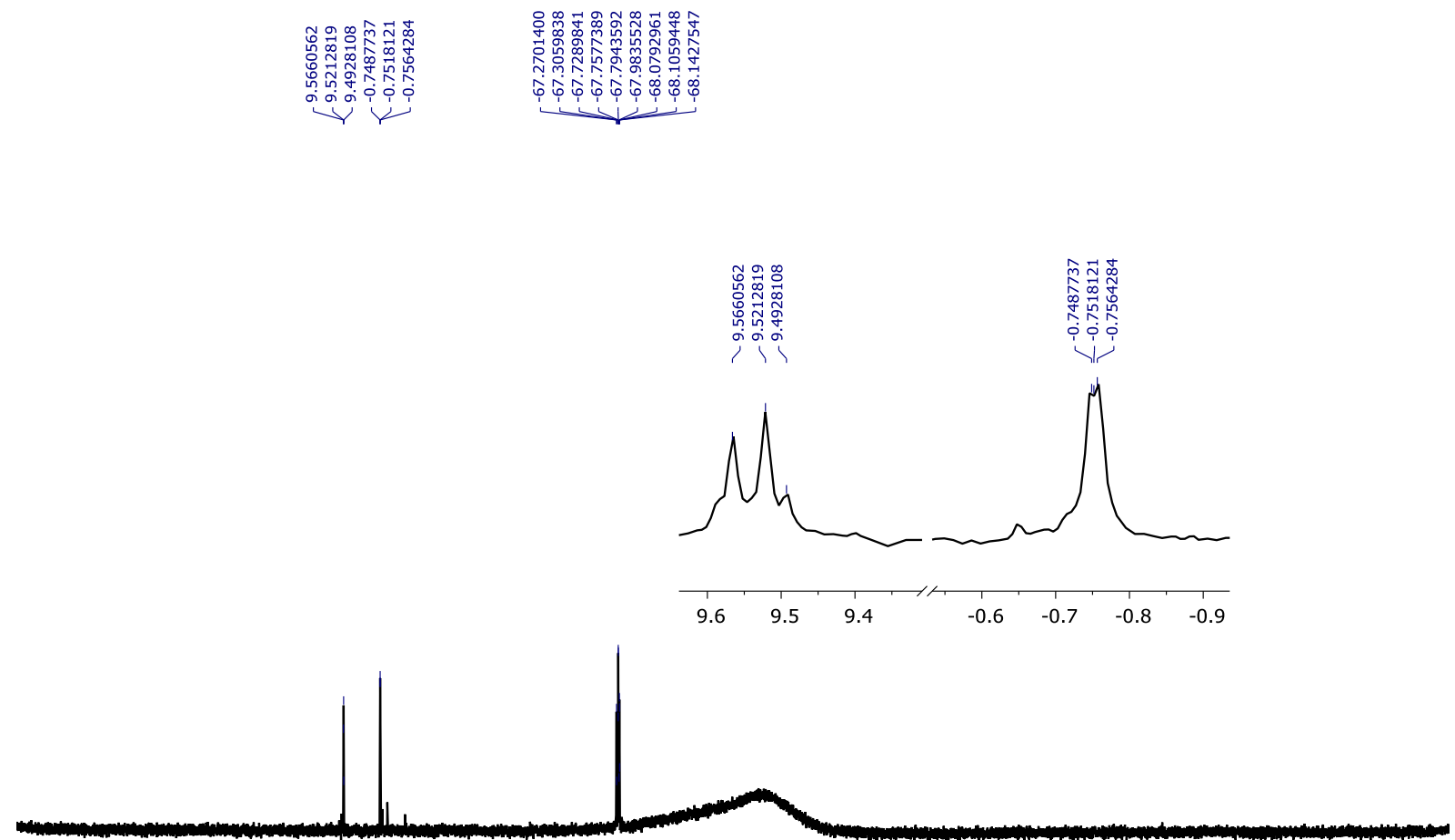

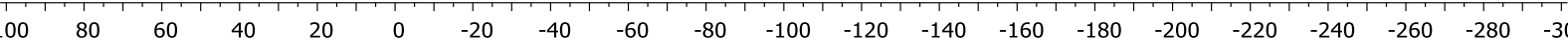


MALDI-TOF MS

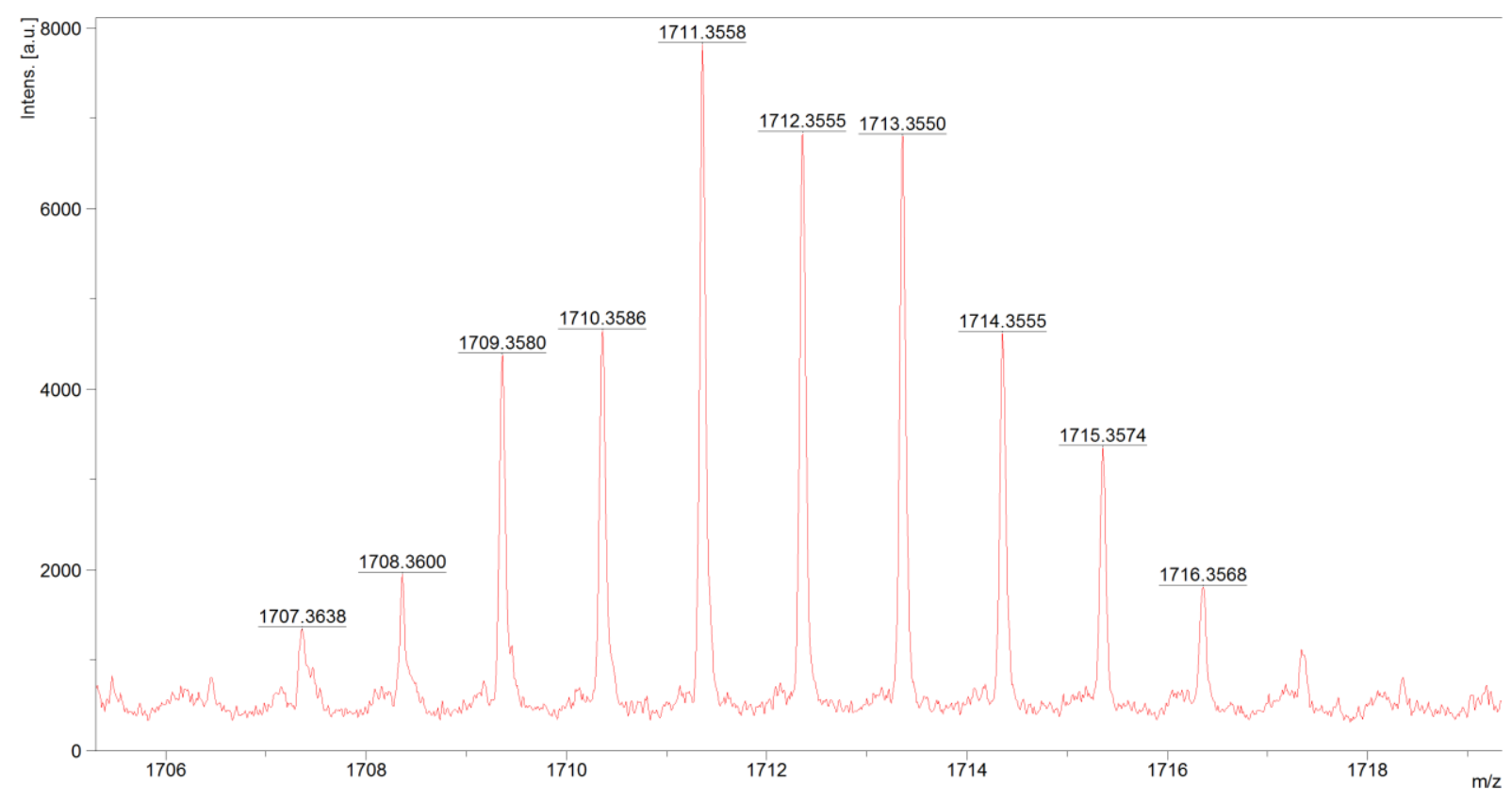


Compound $2 d$

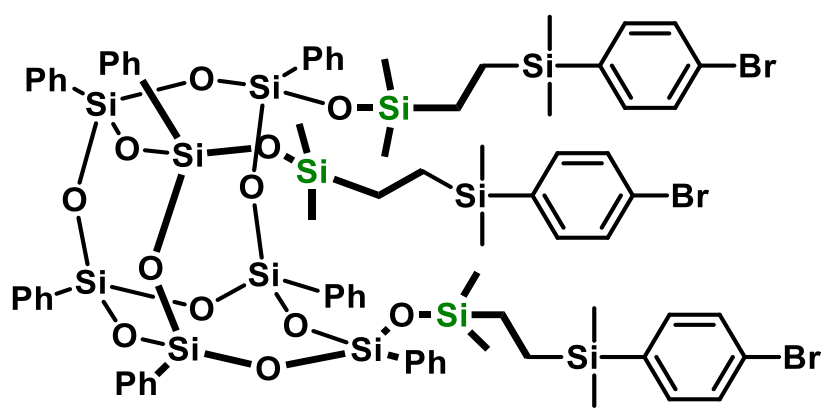

Chemical Formula: $\mathrm{C}_{78} \mathrm{H}_{95} \mathrm{Br}_{3} \mathrm{O}_{12} \mathrm{Si}_{13}$

${ }^{1} \mathrm{H}$ NMR (400 MHz, Chloroform-d) $\delta 7.53-7.42(\mathrm{~m}, 20 \mathrm{H}), 7.41-7.33(\mathrm{~m}, 2 \mathrm{H}), 7.32-7.27(\mathrm{~m}, 7 \mathrm{H})$, $0.71-0.64(\mathrm{~m}, 5 \mathrm{H}), 0.62-0.58(\mathrm{~m}, 2 \mathrm{H}), 0.57-0.49(\mathrm{~m}, 5 \mathrm{H}), 0.30(\mathrm{~s}, 18 \mathrm{H}), 0.21(\mathrm{~s}, 18 \mathrm{H})$.

${ }^{13} \mathrm{C}$ NMR $\left(101 \mathrm{MHz}, \mathrm{CDCl}_{3}\right) \delta 138.30,135.36,134.13,134.09,130.93,128.00,127.74,127.71,127.66$, $123.62,10.14,6.99,-0.16,-3.57$.

${ }^{29} \mathrm{Si} \mathrm{NMR}\left(79 \mathrm{MHz}, \mathrm{CDCl}_{3}\right) \delta$ 12.25, -0.76, -77.37, -77.84, -78.14.

\section{${ }^{1} \mathrm{H}$ NMR $\left(300 \mathrm{MHz}, \mathrm{CDCl}_{3}\right)$}
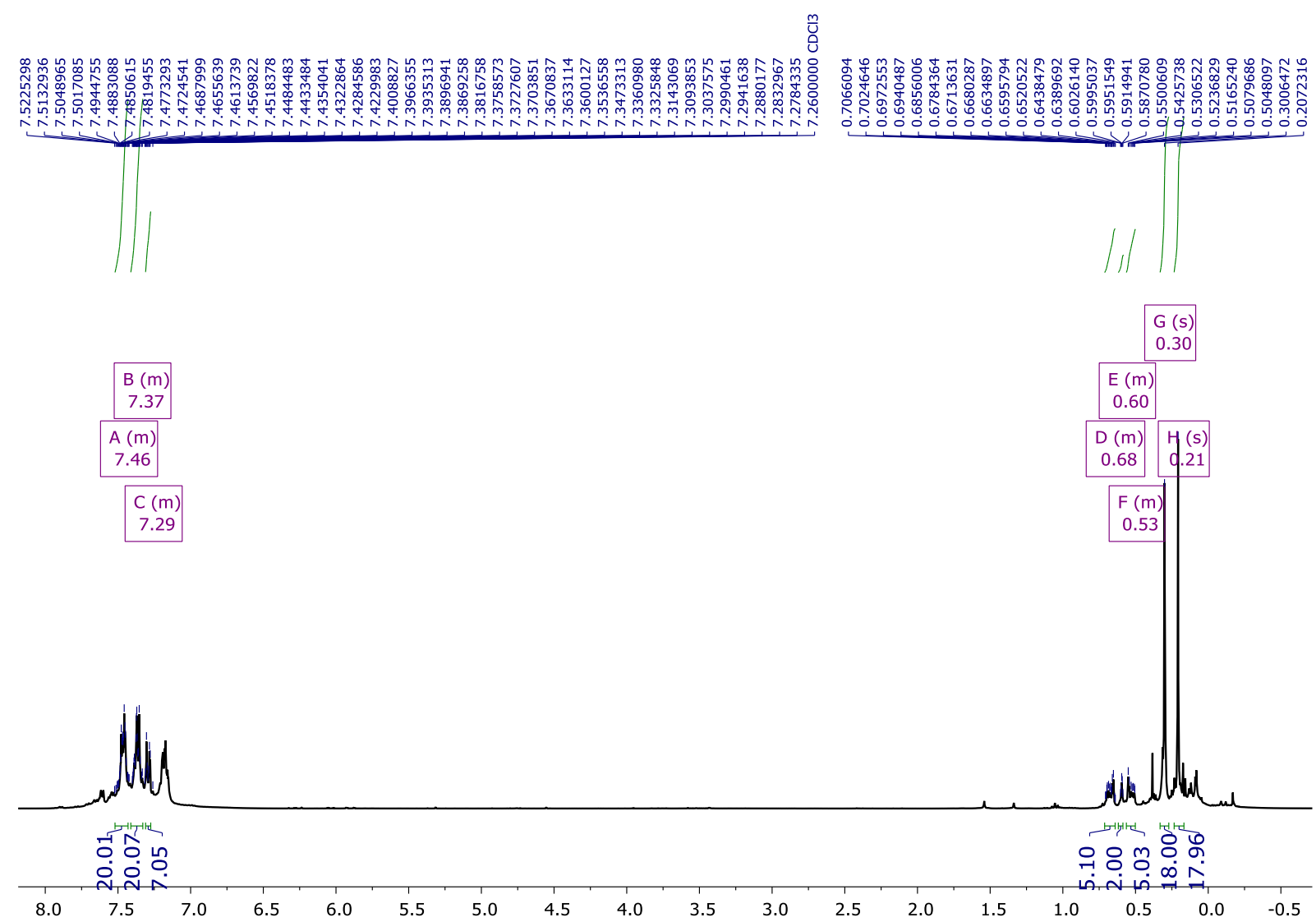
${ }^{13} \mathrm{C}$ NMR (101 MHz, $\mathrm{CDCl}_{3}$ )

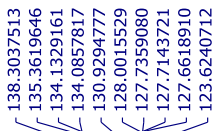

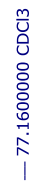
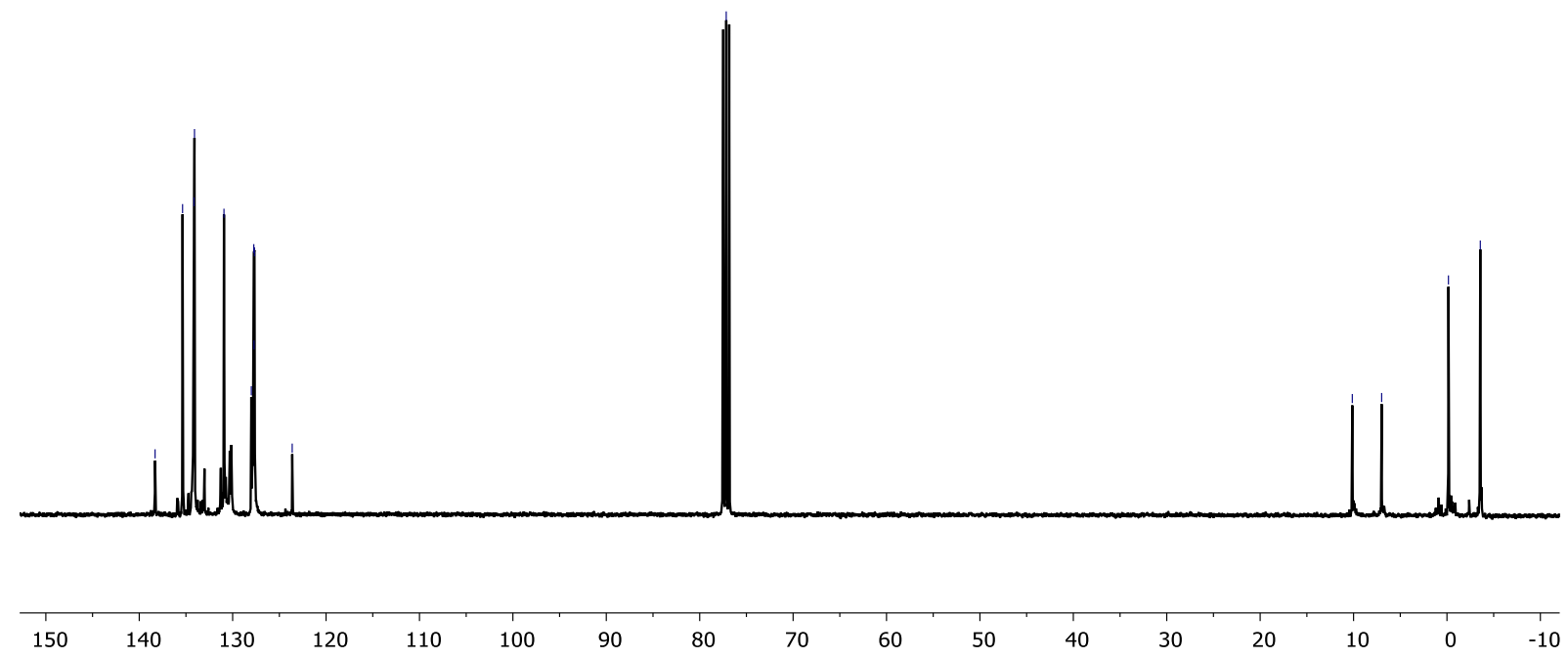

$\left.{ }^{29} \mathrm{Si} \mathrm{NMR} \mathrm{(79} \mathrm{MHz,} \mathrm{CDCl}_{3}\right)$
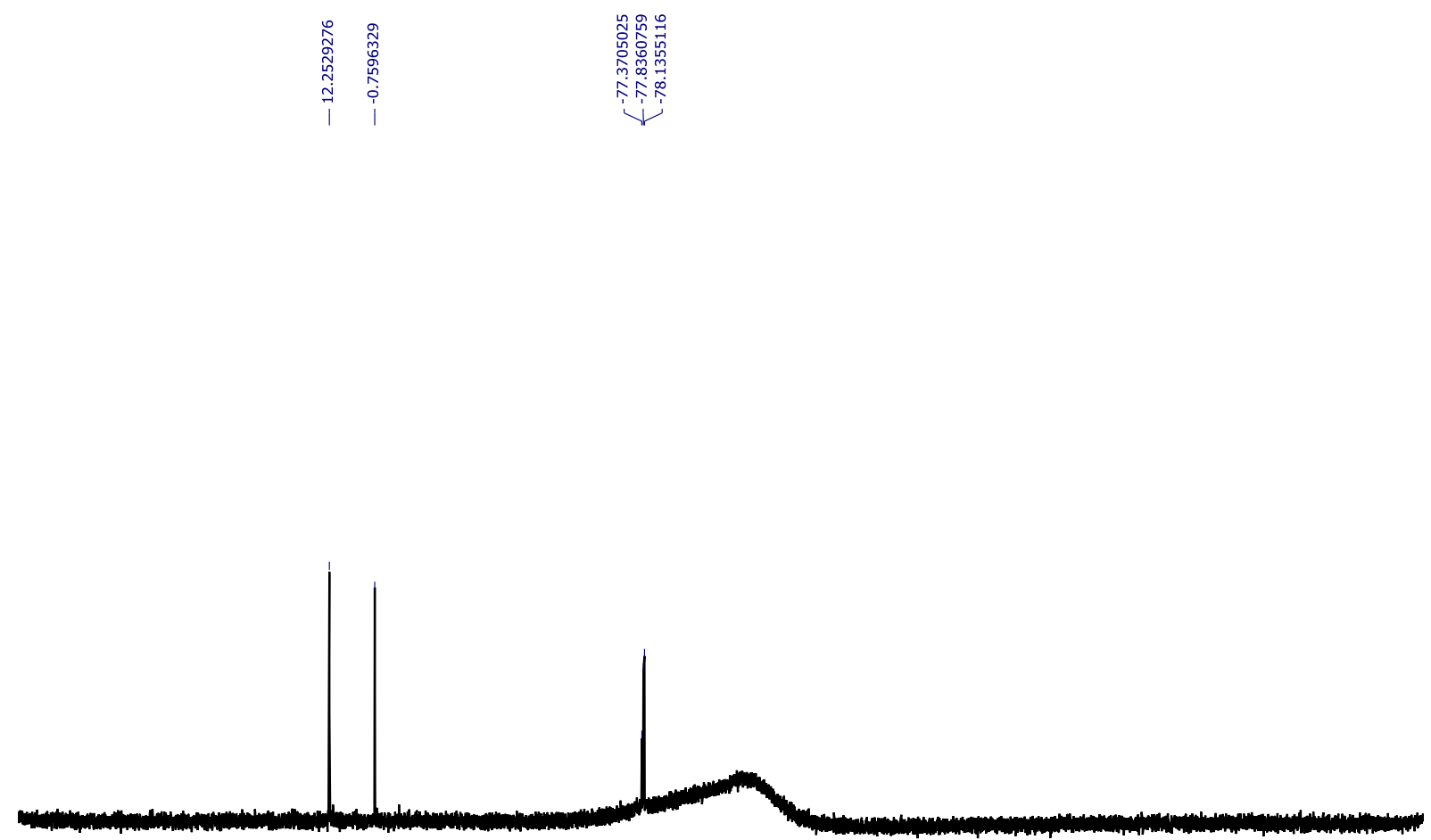

$\begin{array}{lllllllllllllllllllllllllllllllllll}00 & 80 & 60 & 40 & 20 & 0 & -20 & -40 & -60 & -80 & -100 & -120 & -140 & -160 & -180 & -200 & -220 & -240 & -260 & -280 & -31\end{array}$ 
Compound 3d

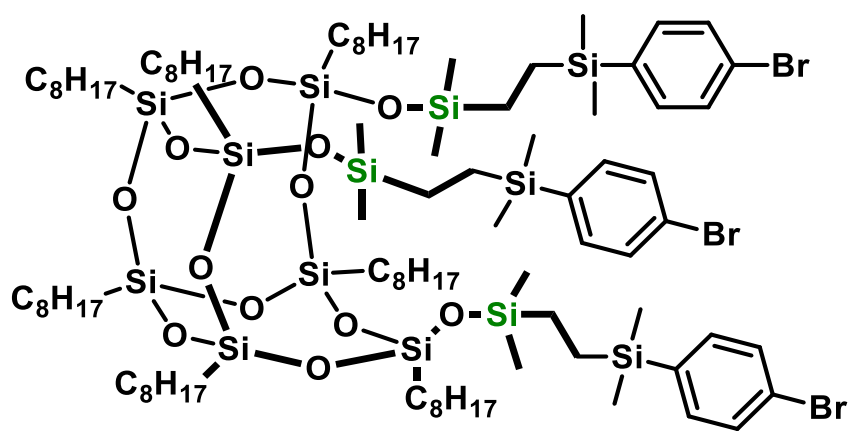

Chemical Formula: $\mathrm{C}_{92} \mathrm{H}_{179} \mathrm{Br}_{3} \mathrm{O}_{12} \mathrm{Si}_{13}$

${ }^{1} \mathrm{H}$ NMR (400 MHz, Chloroform-d) $\delta 7.48(\mathrm{~d}, J=7.3 \mathrm{~Hz}, 6 \mathrm{H}), 7.36(\mathrm{~d}, J=7.8 \mathrm{~Hz}, 6 \mathrm{H}), 1.91-1.74(\mathrm{~m}, 7 \mathrm{H})$, $1.38-1.22(\mathrm{~m}, 7 \mathrm{H}), 1.21-1.13(\mathrm{~m}, 7 \mathrm{H}), 1.08-0.97(\mathrm{~m}, 18 \mathrm{H}), 0.91(\mathrm{~d}, J=4.3 \mathrm{~Hz}, 66 \mathrm{H}), 0.83-0.61(\mathrm{~m}$, $13 \mathrm{H}), 0.60-0.44(\mathrm{~m}, 13 \mathrm{H}), 0.24(\mathrm{~s}, 18 \mathrm{H}), 0.12(\mathrm{~s}, 18 \mathrm{H})$.

${ }^{13} \mathrm{C}$ NMR $\left(101 \mathrm{MHz}, \mathrm{CDCl}_{3}\right) \delta 135.40,131.00,55.23,54.60,31.35,31.32,30.49,30.46,30.39,30.33$, $30.31,29.63,26.56,25.87,25.49,25.38,25.26,25.17,25.06,23.48,10.20,7.18,-0.15,-3.45$.

${ }^{29} \mathrm{Si}$ NMR $\left(79 \mathrm{MHz}, \mathrm{CDCl}_{3}\right)$ $\delta 9.53,9.47,9.36,-0.74,-67.48,-67.75,-67.93,-68.01,-68.21,-68.28$.

MALDI-TOF MS (m/z): calcd. for $\mathrm{C}_{92} \mathrm{H}_{179} \mathrm{Br}_{3} \mathrm{O}_{12} \mathrm{Si}_{13} \mathrm{Na} 2105,24$; found 2104,79.

\section{${ }^{1} \mathrm{H}$ NMR $\left(300 \mathrm{MHz}, \mathrm{CDCl}_{3}\right)$}
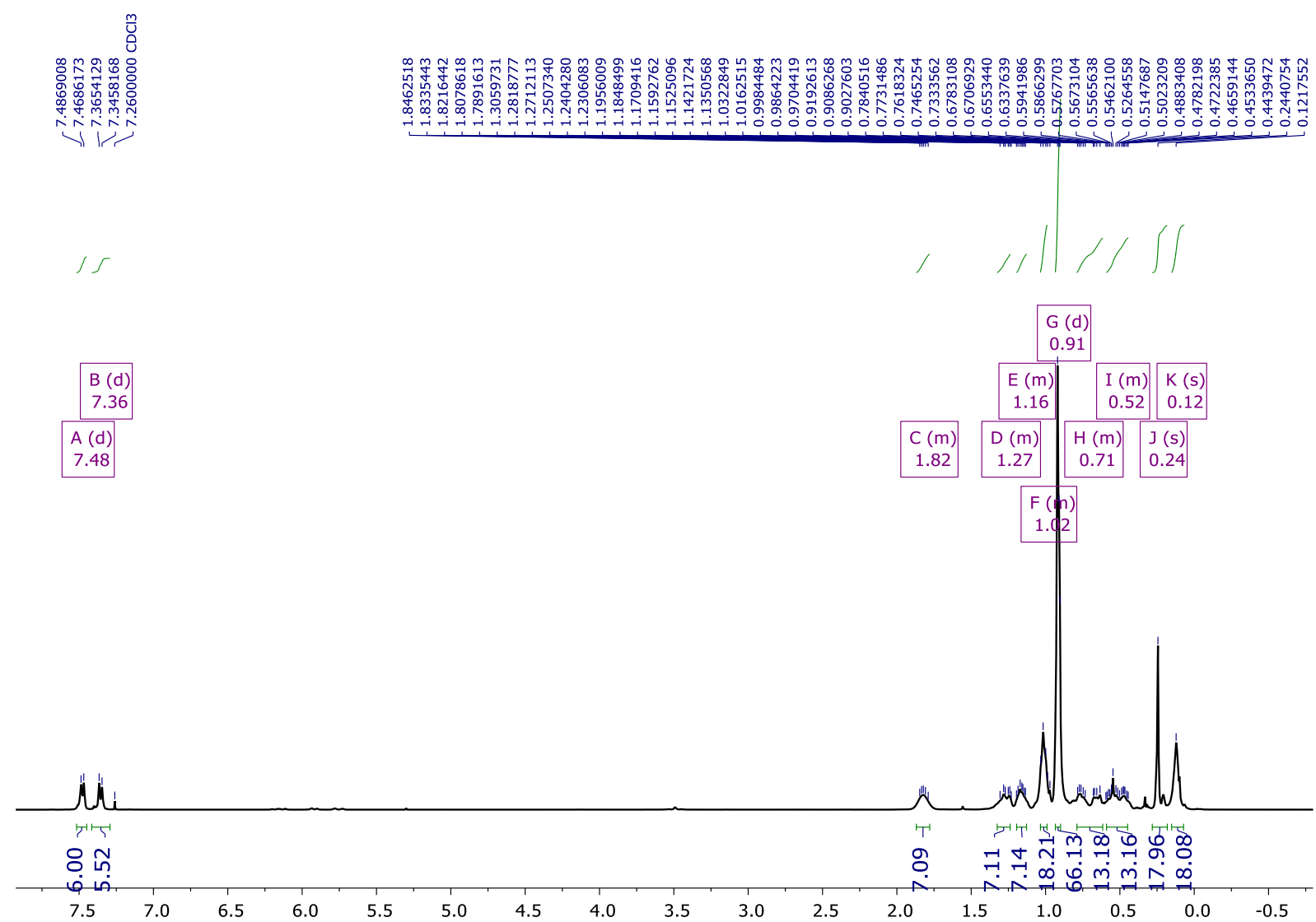
$\left.{ }^{13} \mathrm{C} \mathrm{NMR} \mathrm{(101} \mathrm{MHz,} \mathrm{CDCl}_{3}\right)$
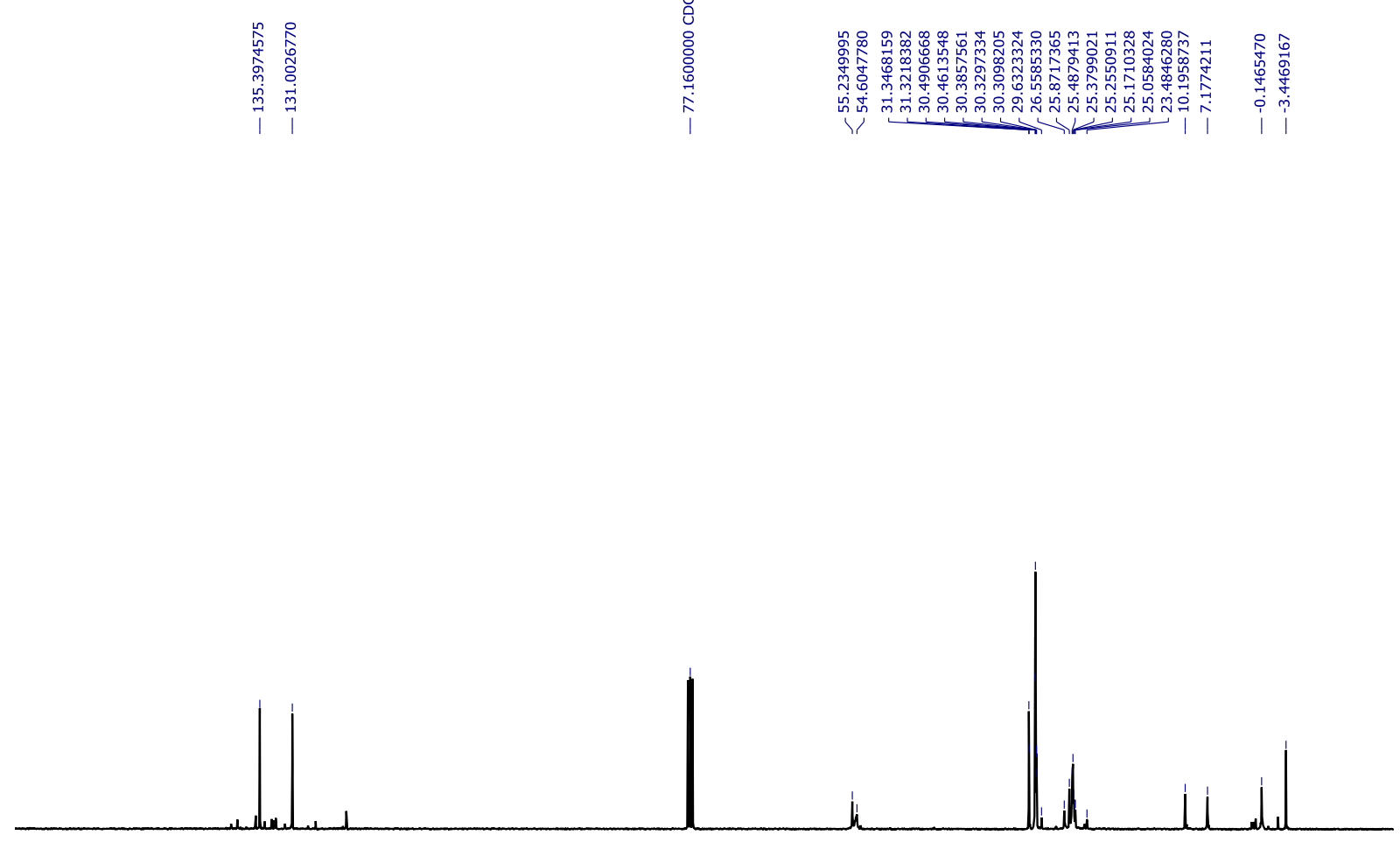

$\begin{array}{lllll}160 & 150 & 140 & 130 & 120\end{array}$

${ }^{29} \mathrm{Si} \mathrm{NMR}\left(79 \mathrm{MHz}, \mathrm{CDCl}_{3}\right)$
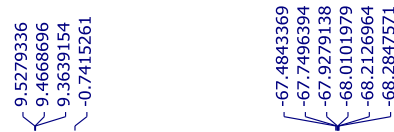

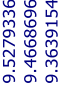

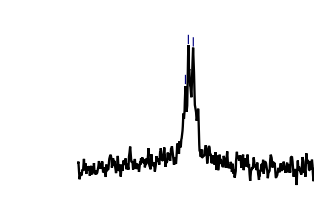

$\begin{array}{llllll}11 & 10 & 9 & 8 & 7\end{array}$

$\begin{array}{lllllllllllllllllllllllll}00 & 80 & 60 & 40 & 20 & 0 & -20 & -40 & -60 & -80 & -100 & -120 & -140 & -160 & -180 & -200 & -220 & -240 & -260 & -280 & -31\end{array}$ 
MALDI-TOF MS

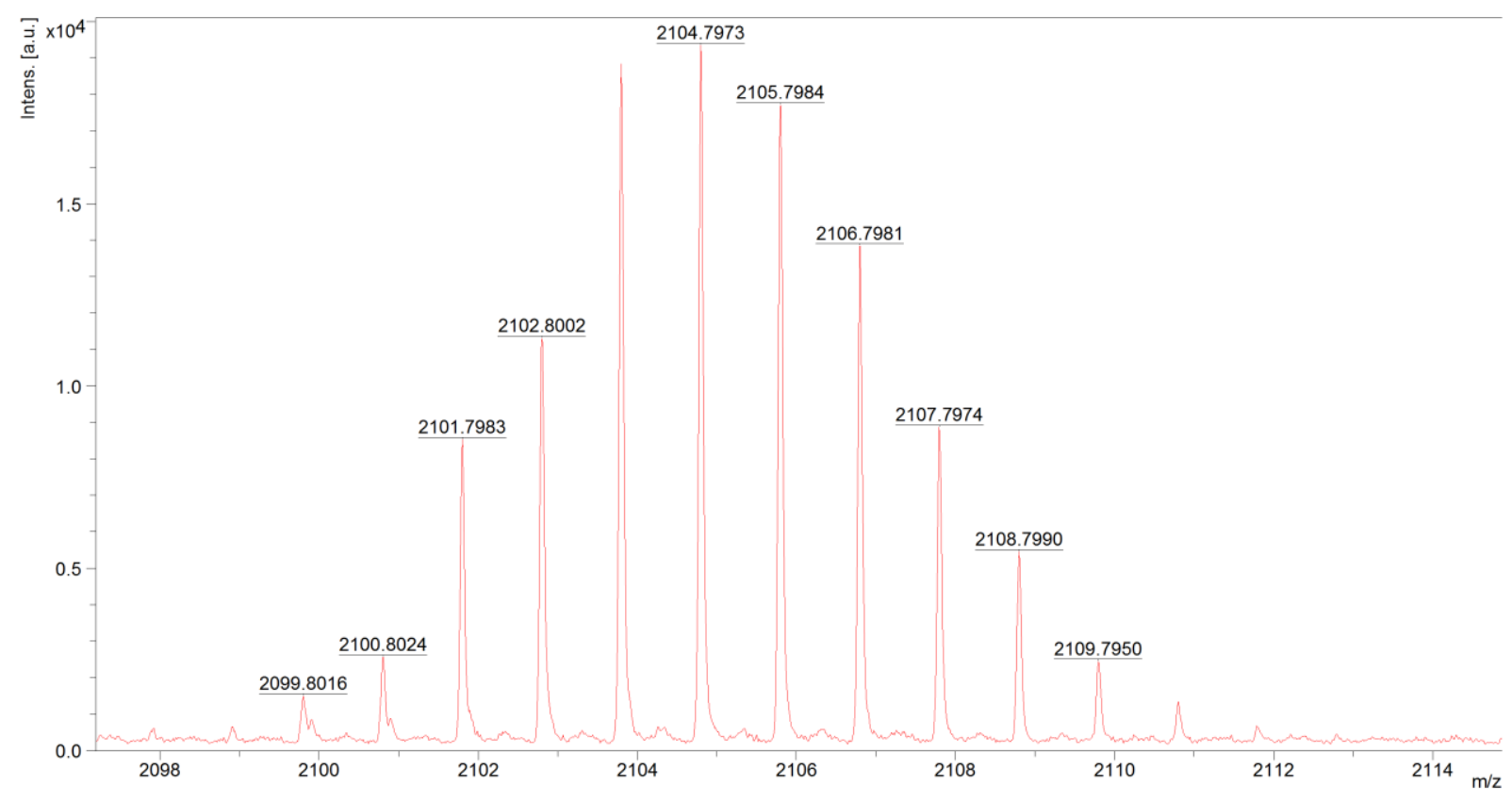


Compound $1 \mathrm{e}$

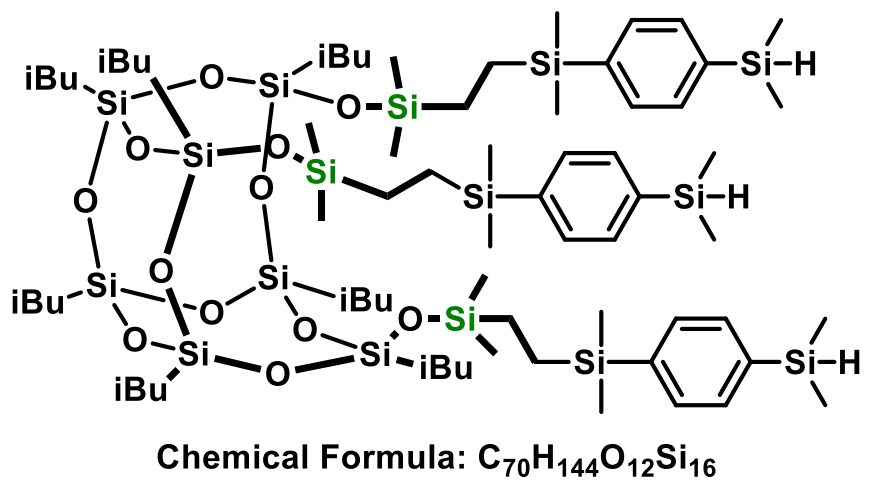

${ }^{1} \mathrm{H}$ NMR (300 MHz, Chloroform-d) $\delta 7.59-7.41(\mathrm{~m}, 12 \mathrm{H}), 4.82-3.86(\mathrm{~m}, 3 \mathrm{H}), 1.91-1.76(\mathrm{~m}, 7 \mathrm{H}), 0.95$ $(\mathrm{dt}, J=6.6,2.0 \mathrm{~Hz}, 42 \mathrm{H}), 0.77-0.64(\mathrm{~m}, 6 \mathrm{H}), 0.61-0.44(\mathrm{~m}, 20 \mathrm{H}), 0.36(\mathrm{t}, J=3.7 \mathrm{~Hz}, 16 \mathrm{H}), 0.32-0.21$ (m, 18H), $0.12(\mathrm{~d}, J=1.9 \mathrm{~Hz}, 14 \mathrm{H}), 0.01(\mathrm{~d}, J=3.5 \mathrm{~Hz}, 4 \mathrm{H}),-0.05(\mathrm{~s}, 2 \mathrm{H})$.

${ }^{13} \mathrm{C} \mathrm{NMR}\left(101 \mathrm{MHz}, \mathrm{CDCl}_{3}\right) \delta 140.85,140.17,139.28,138.69,137.93,133.54,133.38,133.19,133.14$, $133.01,26.25,26.23,26.20,26.14,26.07,26.02$, 25.97, 25.79, 25.38, 25.22, 24.25, 24.22, 24.14, 24.11, $24.02,23.99,23.93,23.84,22.79,22.62,10.23,9.52,7.55,7.10,-0.20,-0.51,-0.85,-3.47,-3.70$.

${ }^{29} \mathrm{Si} \mathrm{NMR}\left(79 \mathrm{MHz}, \mathrm{CDCl}_{3}\right)$ $89.96,9.66,9.29,-0.97,-1.37,-1.58,-17.11,-17.31,-67.30,-67.78,-68.05,-$ 68.16, -68.69.

\section{${ }^{1} \mathrm{H}$ NMR $\left(300 \mathrm{MHz}, \mathrm{CDCl}_{3}\right)$}
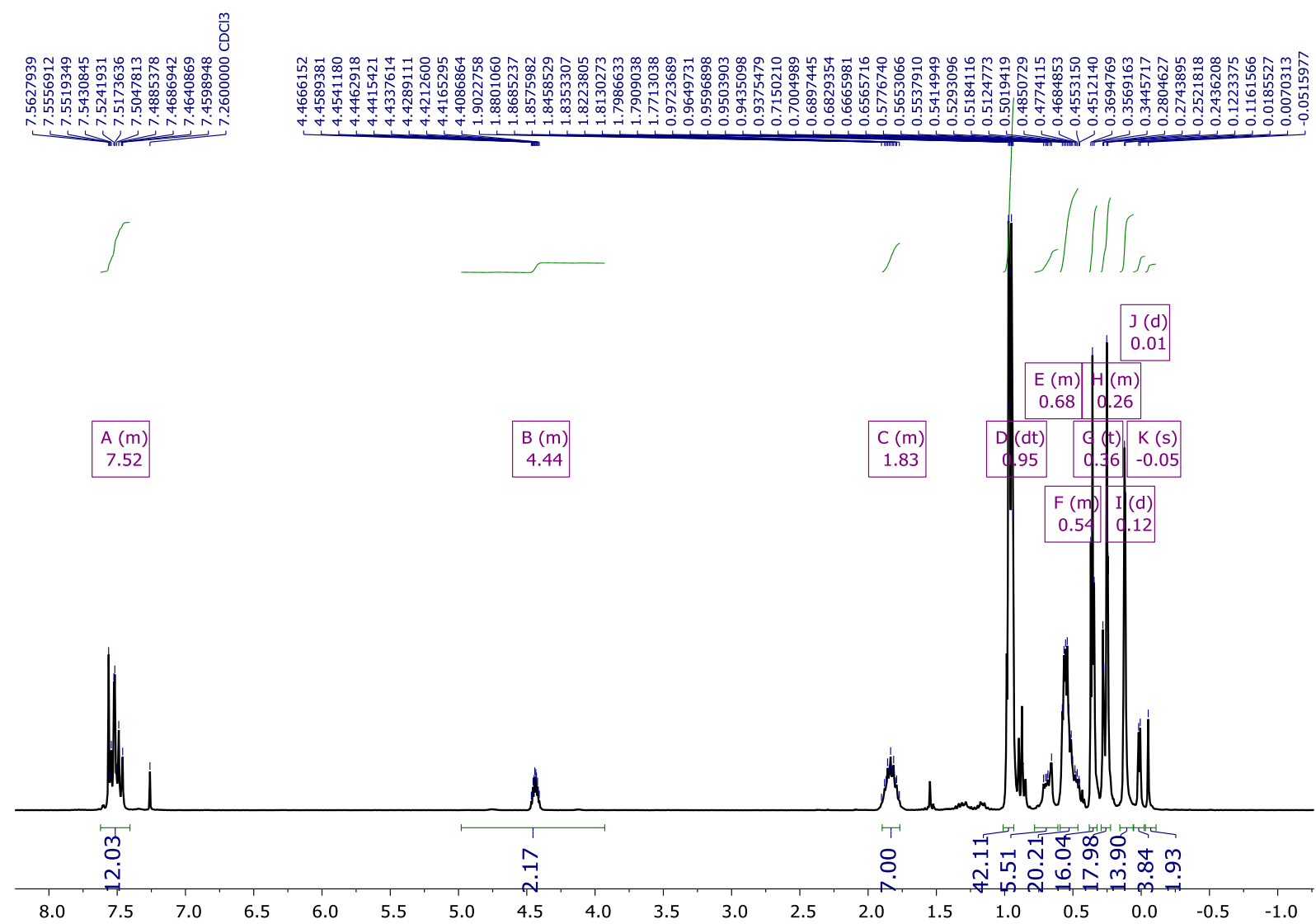
${ }^{13} \mathrm{C}$ NMR (101 MHz, $\mathrm{CDCl}_{3}$ )

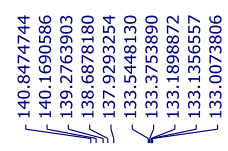

0
0
8
0
0
$i$
$i$
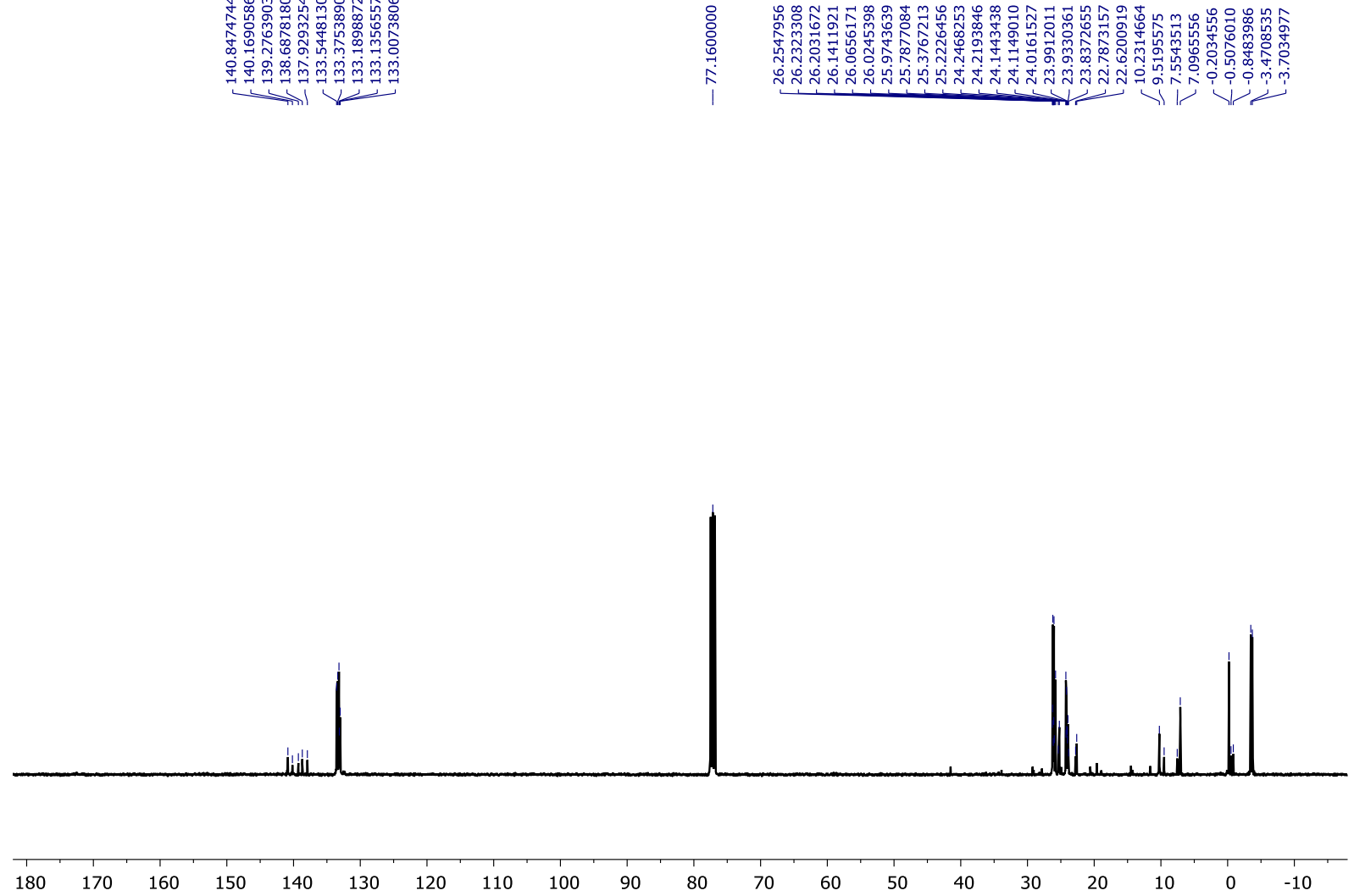

${ }^{29} \mathrm{Si}$ NMR (79 MHz, $\mathrm{CDCl}_{3}$ )
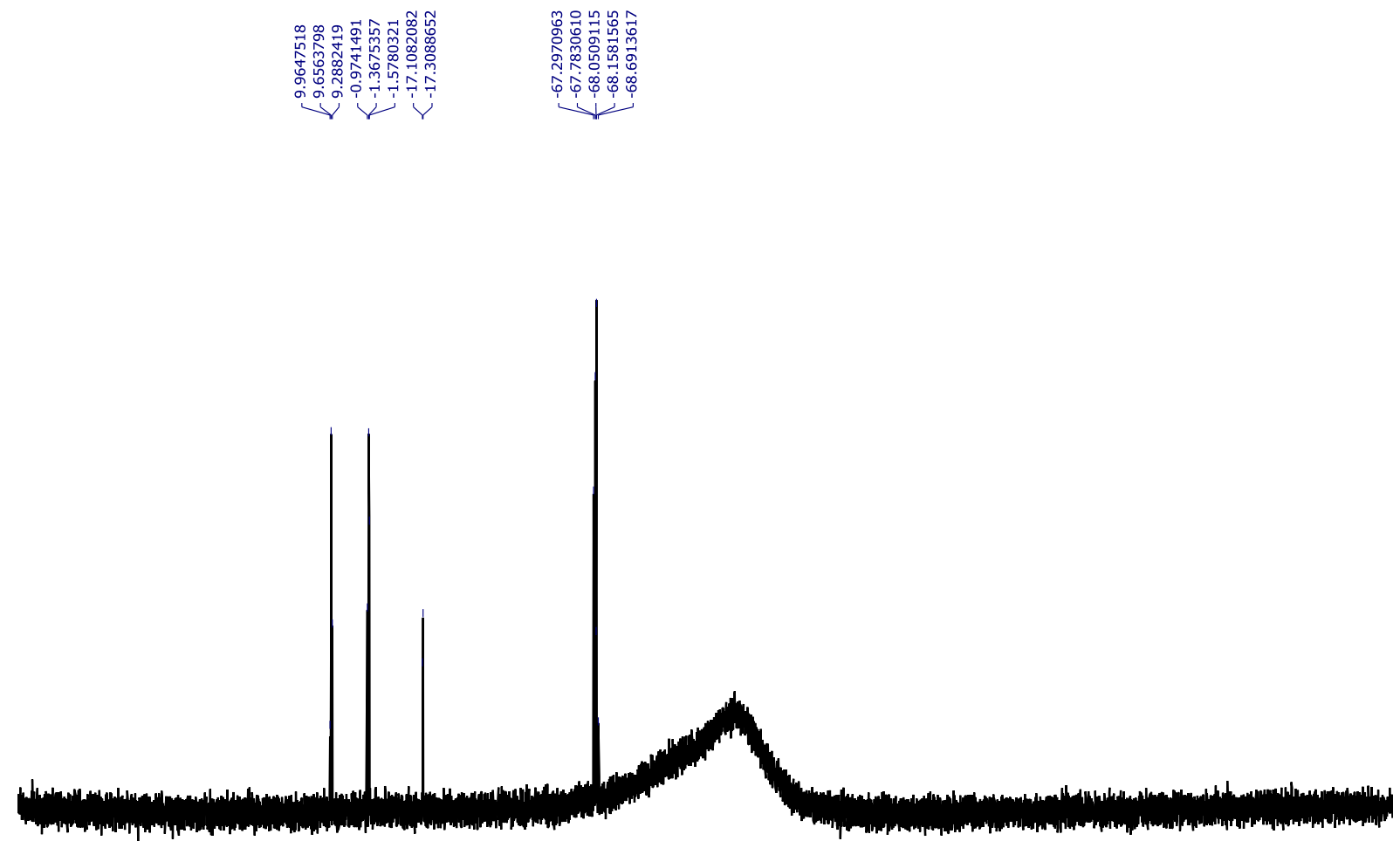

$\begin{array}{llllllllllllllllllllllll}00 & 80 & 60 & 40 & 20 & 0 & -20 & -40 & -60 & -80 & -100 & -120 & -140 & -160 & -180 & -200 & -220 & -240 & -260 & -280 & -31\end{array}$ 
Compound 2e

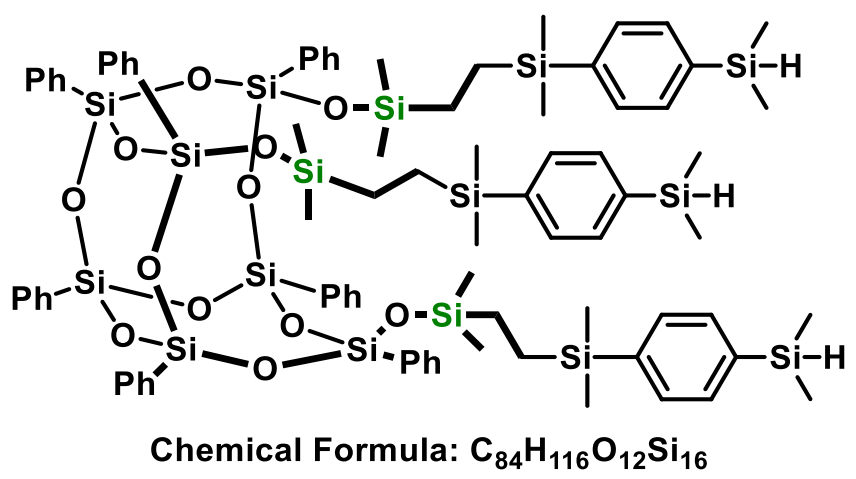

${ }^{1} \mathrm{H}$ NMR (300 MHz, Chloroform-d) $\delta 7.60-7.28(\mathrm{~m}, 38 \mathrm{H}), 7.18-7.06(\mathrm{~m}, 9 \mathrm{H}), 4.54-4.28(\mathrm{~m}, 3 \mathrm{H}), 0.73$ $-0.40(\mathrm{~m}, 12 \mathrm{H}), 0.39-0.29(\mathrm{~m}, 2 \mathrm{H}), 0.27-0.21(\mathrm{~m}, 16 \mathrm{H}), 0.15(\mathrm{~d}, J=4.7 \mathrm{~Hz}, 10 \mathrm{H}), 0.02(\mathrm{~d}, J=9.6 \mathrm{~Hz}$, $8 \mathrm{H})$.

${ }^{13} \mathrm{C}$ NMR $\left(101 \mathrm{MHz}, \mathrm{CDCl}_{3}\right) \delta 134.16,134.13,134.10,133.54,133.37,133.34,133.23,133.21,133.17$, 132.34, 131.34, 130.22, 130.05, 127.98, 127.96, 127.79, 127.69, 127.64, 127.61, 10.20, 9.62, 7.52, 7.01, $-0.15,-3.57,-3.70$.

${ }^{29} \mathrm{Si} \mathrm{NMR}\left(79 \mathrm{MHz}, \mathrm{CDCl}_{3}\right) \delta$ 12.53, 12.34, 12.01, -0.83, -1.35, -1.40, -17.33, -77.42, -77.92, -78.19.

\section{${ }^{1} \mathrm{H}$ NMR $\left(300 \mathrm{MHz}, \mathrm{CDCl}_{3}\right)$}
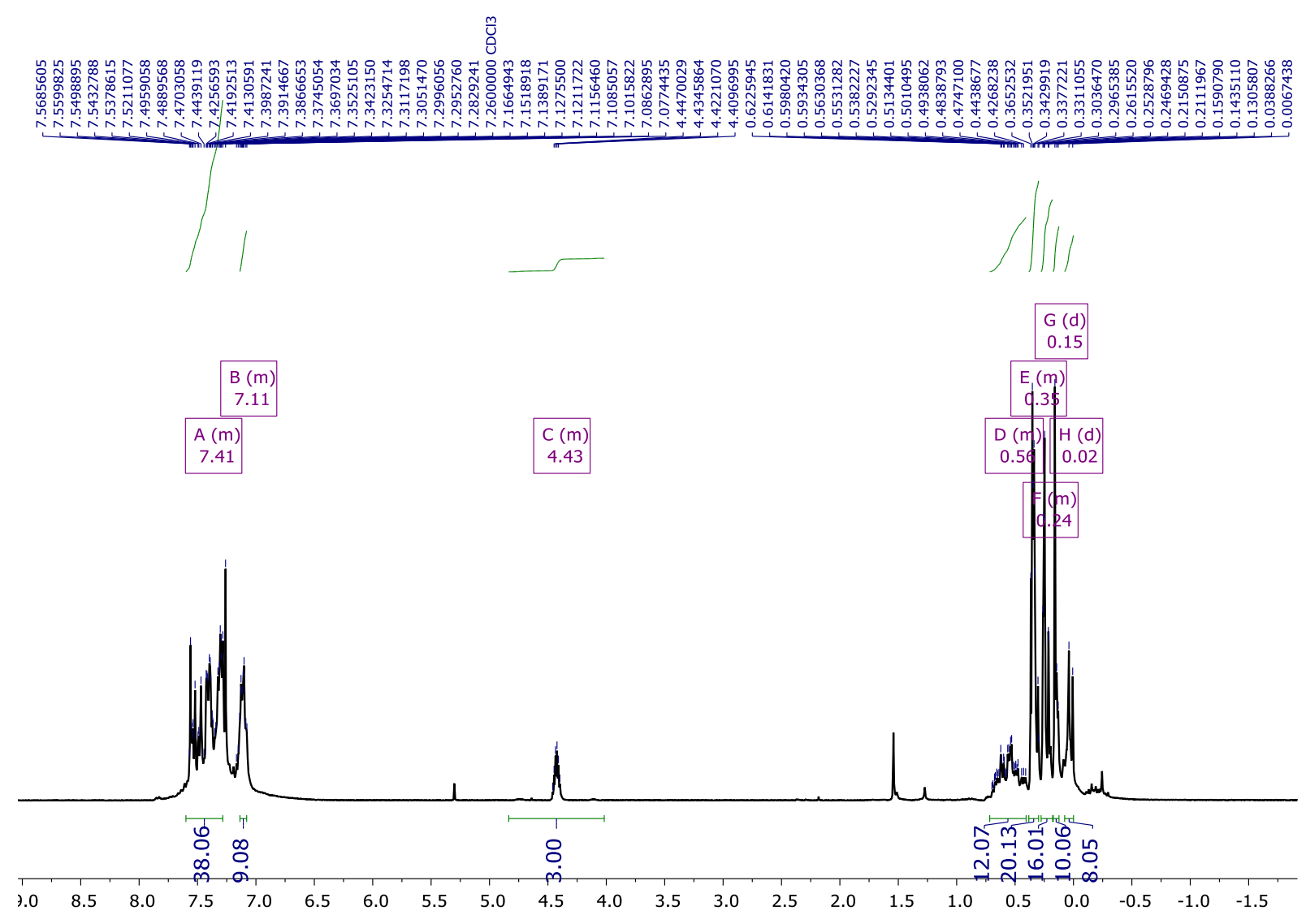
${ }^{13} \mathrm{C}$ NMR $\left(101 \mathrm{MHz}, \mathrm{CDCl}_{3}\right)$

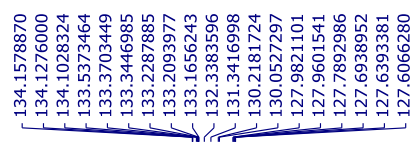

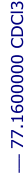
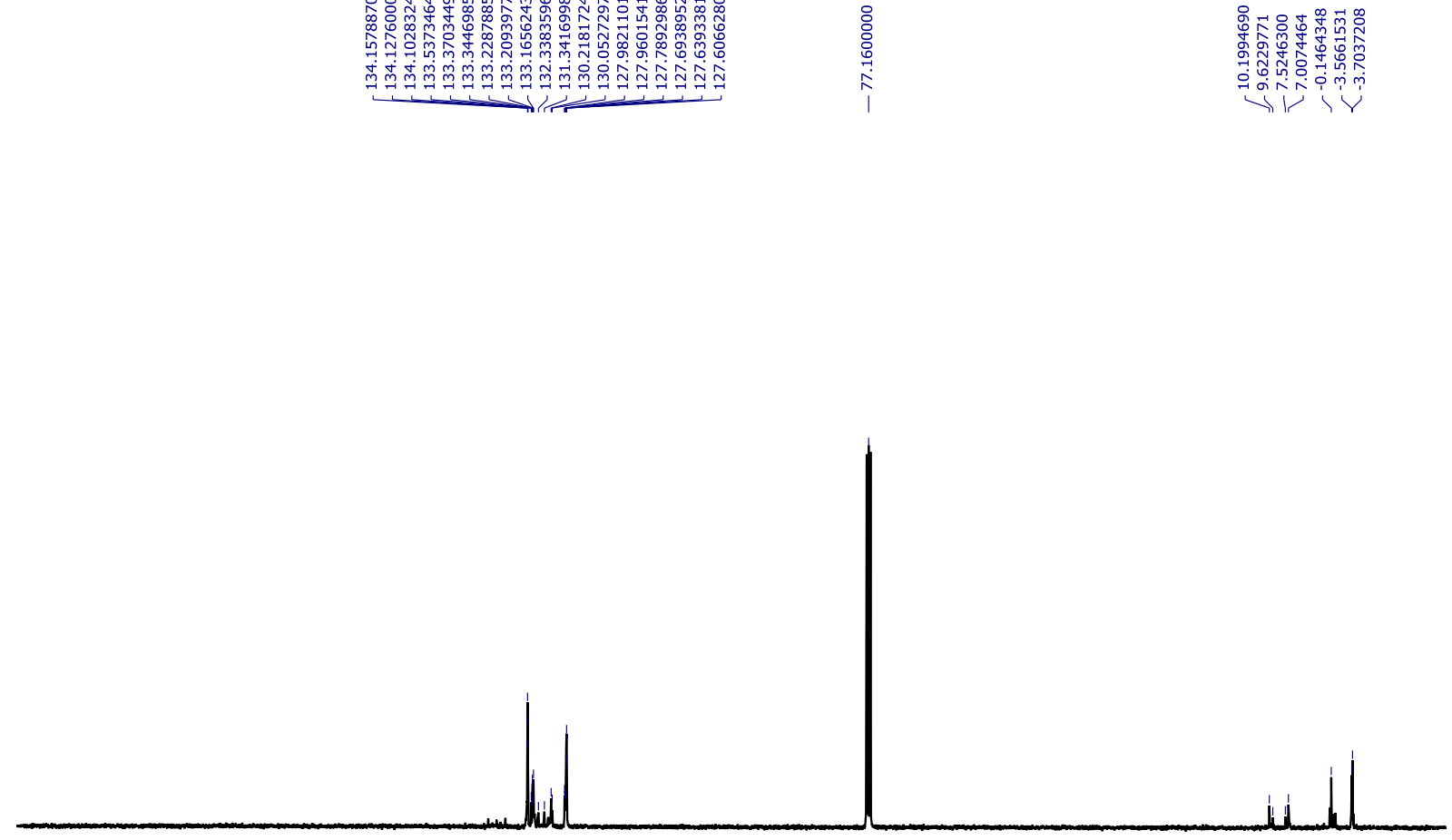

$\begin{array}{lllllllllllllllllllllll}210 & 200 & 190 & 180 & 170 & 160 & 150 & 140 & 130 & 120 & 110 & 100 & 90 & 80 & 70 & 60 & 50 & 40 & 30 & 20 & 10 & 0 & -10\end{array}$

${ }^{29} \mathrm{Si}$ NMR $\left(79 \mathrm{MHz}, \mathrm{CDCl}_{3}\right)$
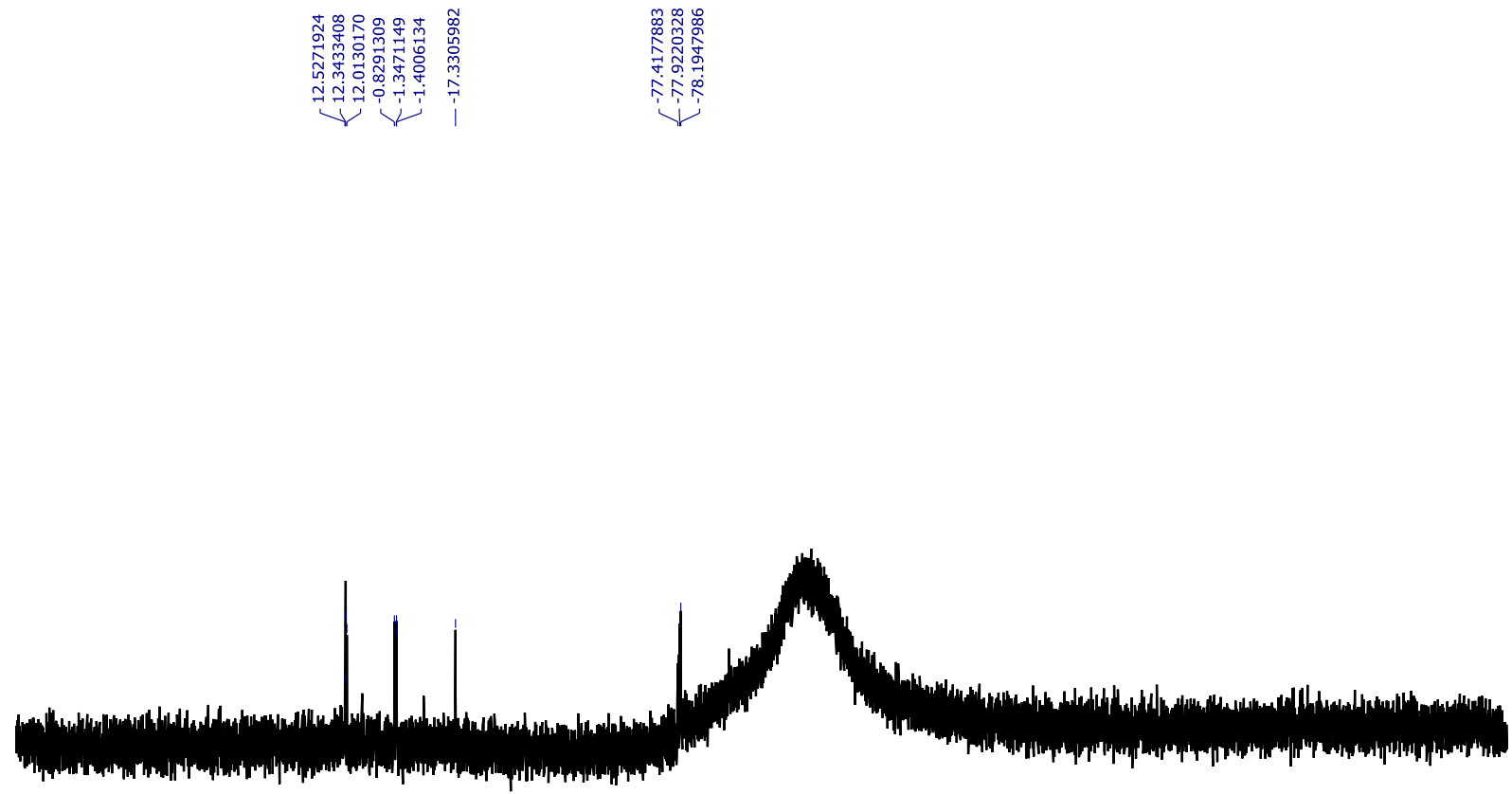

$\begin{array}{lllllllllllllllllllllllllllllllll}.00 & 80 & 60 & 40 & 20 & 0 & -20 & -40 & -60 & -80 & -100 & -120 & -140 & -160 & -180 & -200 & -220 & -240 & -260 & -280 & -31\end{array}$ 
6. TGA curves obtained for selected compounds 1-9

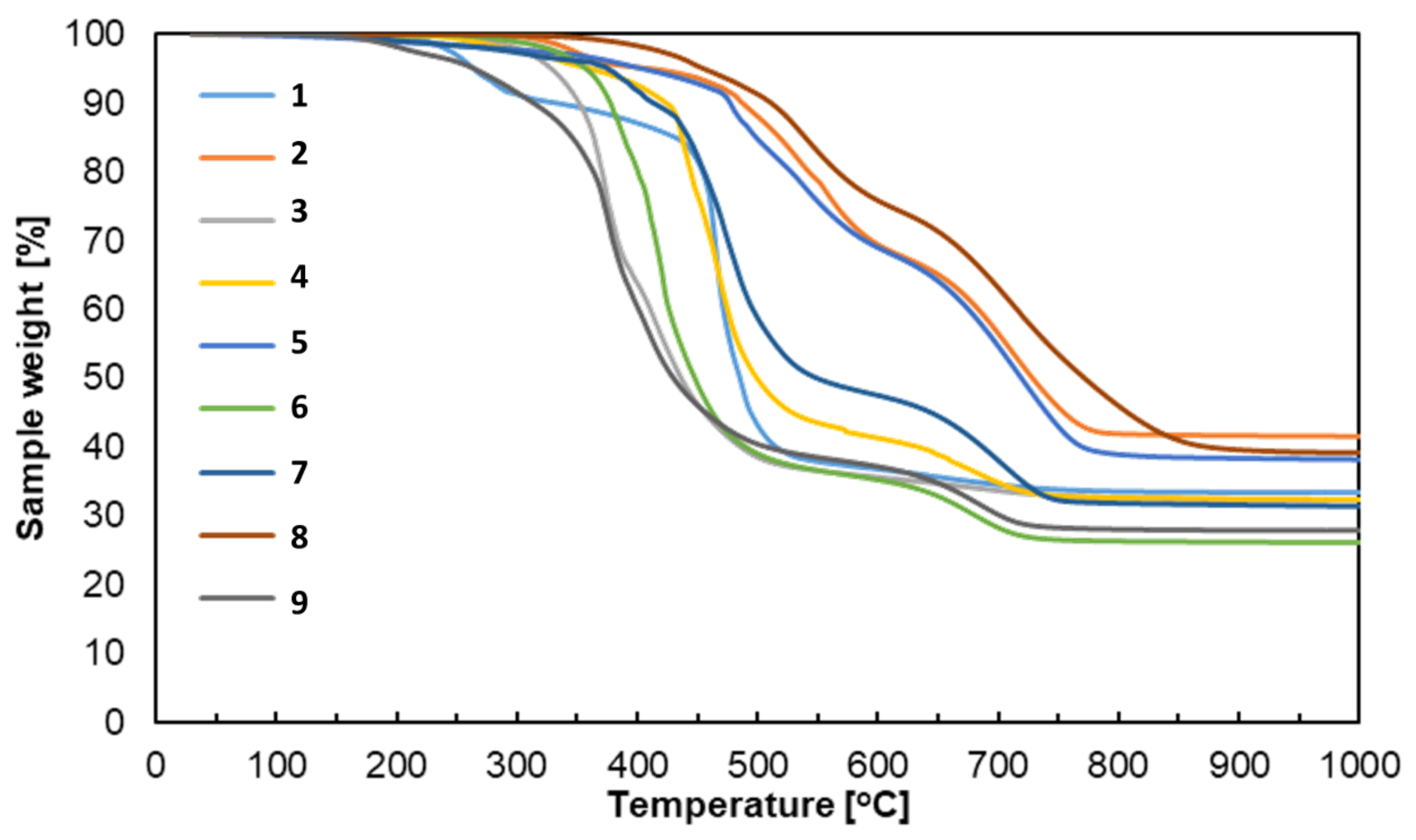

